"ATMP-16-3-A5-GAI" — 2013/2/1 — 19:36 — page 935 — \#1

(C) 2012 International Press

Adv. Theor. Math. Phys. 16 (2012) 935-1086

\title{
Knot invariants from
}

\section{four-dimensional gauge theory}

\author{
Davide Gaiotto $^{1}$ and Edward Witten ${ }^{1,2}$
}

${ }^{1}$ School of Natural Sciences, Institute for Advanced Study, 1 Einstein Drive, Princeton, NJ 08540, USA

dgaiotto@perimeterinstitute.ca

${ }^{2}$ Department of Physics, Stanford University, Palo Alto, CA 94305, USA

\begin{abstract}
It has been argued based on electric-magnetic duality and other ingredients that the Jones polynomial of a knot in three dimensions can be computed by counting the solutions of certain gauge theory equations in four dimensions. Here, we attempt to verify this directly by analyzing the equations and counting their solutions, without reference to any quantum dualities. After suitably perturbing the equations to make their behavior more generic, we are able to get a fairly clear understanding of how the Jones polynomial emerges. The main ingredient in the argument is a link between the four-dimensional gauge theory equations in question and conformal blocks for degenerate representations of the Virasoro algebra in two dimensions. Along the way we get a better understanding of how our subject is related to a variety of new and old topics in mathematical physics, ranging from the Bethe ansatz for the Gaudin spin chain to the $M$-theory description of Bogomol'nyi-Prasad-Sommerfield (BPS) monopoles and the relation between Chern-Simons gauge theory and Virasoro conformal blocks.
\end{abstract}

e-print archive: http://lanl.arXiv.org/abs/1106.4789v1 
1 Introduction $\quad 939$

$\begin{array}{lll}1.1 & \text { A brief review } & 940\end{array}$

1.1.1 Lift to Khovanov homology 943

$\begin{array}{lll}1.2 & \text { Methods used in this paper } & 945\end{array}$

$\begin{array}{lll}1.3 & \text { Outline and results } & 948\end{array}$

2 Analysis at $t=1 \quad 948$

$\begin{array}{lll}2.1 & \text { Some preliminaries } & 948\end{array}$

$\begin{array}{lll}2.2 & \text { The boundary condition } & 952\end{array}$

$\begin{array}{ll}\text { 2.2.1 Adding singular monopoles } & 955\end{array}$

$\begin{array}{lll}2.3 & \text { Solutions without symmetry breaking } & 957\end{array}$

2.4 "Real" symmetry breaking $\quad 959$

2.5 "Complex" symmetry breaking 962

2.5.1 Implications $\quad 964$

3 Analysis at general $t \quad 965$

$\begin{array}{lll}3.1 & \text { Some basics } & 965\end{array}$

$\begin{array}{lll}3.2 & \text { Nahm poles and opers } & 969\end{array}$

$\begin{array}{lll}\text { 3.2.1 Some further remarks } & 971\end{array}$

$\begin{array}{lll}3.3 & \text { Opers with singularities } & 972\end{array}$

3.4 Oper singularities and Bethe equations $\quad 973$

$\begin{array}{lll}3.4 .1 & \text { Relation to conformal field theory } & 977\end{array}$

$\begin{array}{lll}3.5 & \text { Symmetry breaking again } & 978\end{array}$

$\begin{array}{lll}\text { 3.5.1 "Real" symmetry breaking } & 978\end{array}$ 
3.5.2 "Complex" symmetry breaking 980

$\begin{array}{lll}3.6 & \text { Opers and stress tensors } & 983\end{array}$

$\begin{array}{lll}3.7 & \text { Opers and Virasoro conformal blocks } & 986\end{array}$

$\begin{array}{lll}\text { 3.7.1 } & \text { The irregular case } & 989\end{array}$

3.7.2 Degenerate primary fields and trivial monodromy $\quad 990$

4 Four-dimensional solutions and parallel transport $\quad 992$

$\begin{array}{lll}4.1 & \text { Introduction } & 992\end{array}$

4.1.1 Relation to Morse theory 993

$\begin{array}{lll}\text { 4.1.2 } & \text { Time dependence } & 995\end{array}$

$\begin{array}{lll}\text { 4.1.3 The dual basis } & 998\end{array}$

4.1.4 Non-single valued superpotentials and $q$-grading

4.2 Classical description of counting of fourdimensional solutions

5 From braiding of thimbles to free-field integrals

1003

5.1 From thimbles to integrals

5.2 Two Chern-Simons theories

5.3 From Chern-Simons to conformal blocks 1006

$\begin{array}{lll}\text { 5.3.1 Analog for Liouville } & 1008\end{array}$

5.4 Wilson line operators

5.5 Singular monopoles and "heavy" degenerate fields

5.6 Putting the pieces together 
6.1 Overview

6.1.1 $S U(2)$ versus $S O(3)$

6.2 A single critical point

6.2.1 Braiding of two primaries with minimal charge

6.2.2 One primary field with symmetry breaking

6.3 Two critical points

6.3.1 The first example

6.3.2 The second example 1023

6.4 A final example 1024

6.5 General picture with symmetry breaking 1025

6.5.1 Degenerate fields of any charge

6.6 Turning off symmetry breaking 1027

6.6.1 A clarification 1029

6.7 Three-dimensional interpretation 1030

6.7.1 Creation and annihilation of strands

6.7.2 Some examples and some topological details

6.7.3 Gradient flow and strings

6.7.4 Analog in the dual Chern-Simons theory 1041

6.8 Up to six dimensions

$7 \quad$ An effective superpotential for monopoles 
7.2 Coordinates for monopoles

7.3 Realization via $M$-theory and branes

7.3.1 $M$-theory preliminaries

7.3.2 Reduction to gauge theory

Appendix A Three-dimensional BPS equations from six dimensions

Appendix B Three-dimensional BPS equations from four and eight dimensions

Appendix C On boundary conditions and a special solution of the BPS equations

\section{Introduction}

The Jones polynomial [1] is an invariant of knots that has multiple relations to many aspects of mathematical physics, including integrable lattice 
statistical mechanics, two-dimensional conformal field theory and associated representations of braid groups, and three-dimensional Chern-Simons gauge theory. Khovanov homology [2] is a more recent topological theory in four dimensions; in this theory, a knot is viewed as an object in three-dimensional space and the invariant associated to a knot is a vector space (of physical states) rather than a number. The relation between the two theories is that the four-dimensional theory associated to Khovanov homology, when compactified on a circle, reduces to the three-dimensional theory that yields the Jones polynomial.

Khovanov homology has been interpreted physically [3] in terms of topological strings, building on earlier work on BPS states of open strings [4]. See [5-13] for a sampling of additional developments. An alternative but closely related physical interpretation of Khovanov homology has been given in [14], where more detailed references can be found concerning the Jones polynomial, Khovanov homology, and their relations to mathematical physics.

According to this more recent proposal, the Jones polynomial can be computed by counting the solutions of certain elliptic partial differential equations (PDEs) in four dimensions, and Khovanov homology can then be constructed by counting the solutions of related equations in $(4+1)$ dimensions. The reasoning that led to this proposal relied on electric-magnetic duality of $\mathcal{N}=4$ super Yang-Mills theory in four dimensions to transform one description that is rather "quantum" in nature (being closely related to Chern-Simons gauge theory on a bounding three-manifold) to another that is "semiclassical" in the sense that the partition function can be computed just by suitably counting the classical solutions of certain differential equations.

Instead of relying on electric-magnetic duality to predict this perhaps mysterious result, can we understand it by a direct study of the equations? This is the goal of the present paper. We will gain a reasonable degree of understanding of the Jones polynomial and a good foundation for understanding Khovanov homology.

\subsection{A brief review}

The four-dimensional equations in question can be described as follows. The gauge group is a compact Lie group ${ }^{1} G$. The fields in the equations are a

\footnotetext{
${ }^{1}$ We reverse notation from [14], writing $G^{\vee}$ for the gauge group in the Chern-Simons description and $G$ for the gauge group in the dual "magnetic" description, on which we focus in this paper.
} 


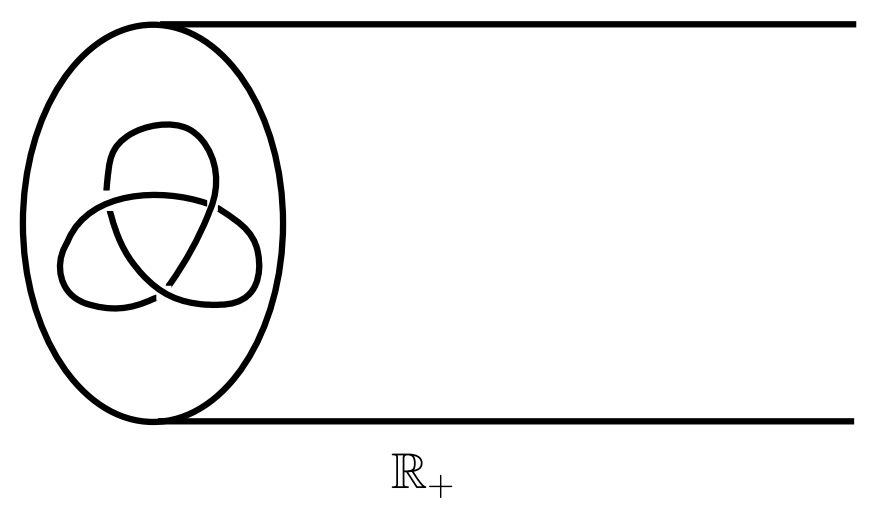

Figure 1: A knot has been placed at the boundary of the four-manifold $M_{4}=W \times \mathbb{R}_{+}$.

gauge field $A$ which is a connection on a $G$-bundle $E \rightarrow M_{4}$, with $M_{4}$ an oriented Riemannian four-manifold, and another field $\phi$ that is a one-form valued in the adjoint representation of $G$. The equations, which were first studied in relation to the geometric Langlands correspondence [15], read

$$
\begin{aligned}
\left(F-\phi \wedge \phi+t d_{A} \phi\right)^{+} & =0, \\
\left(F-\phi \wedge \phi-t^{-1} d_{A} \phi\right)^{-} & =0, \\
d_{A} \star \phi & =0,
\end{aligned}
$$

where the selfdual and anti-selfdual projections of a two-form $b$ are denoted $b^{ \pm} ; d_{A}=d+[A, \cdot]$ is the gauge-covariant exterior derivative; $F=d A+A \wedge$ $A$ is the Yang-Mills field strength; $\star$ is the Hodge star operator; and $t$ is a real parameter. (Actually, $t$ takes values in $\mathbb{R P}^{1}=\mathbb{R} \cup \infty$; for $t \rightarrow 0$ or $t \rightarrow \infty$, one multiples the second equation by $t$ or the first by $t^{-1}$.) To study knot invariants, one specializes to $M_{4}=W \times \mathbb{R}_{+}$, where $W$ is a three-manifold and $\mathbb{R}_{+}$is the half-line $y \geq 0$ (figure 1 ).

The boundary condition at $y=0$ is slightly subtle but can be easily described in the absence of knots. Suppose first that $t=1$. Since the boundary condition is local, we can specialize to $W=\mathbb{R}^{3}$ in describing it. (In any case, that is the main example for the present paper.) Consider a classical solution that is invariant under translations along $\mathbb{R}^{3}$ and such that $A$ and the part of $\phi$ normal to the boundary vanish. The equations then reduce to Nahm's equations for $\vec{\phi}$, the part of $\phi$ tangent to the boundary:

$$
\frac{d \vec{\phi}}{d y}+\vec{\phi} \times \vec{\phi}=0
$$


These equations have a singular solution, first introduced by Nahm in his work on monopoles. Pick an embedding $\rho: \mathfrak{s u}(2) \rightarrow \mathfrak{g}($ where $\mathfrak{s u}(2)$ and $\mathfrak{g}$ are the Lie algebras of $S U(2)$ and $G$, respectively), given by a triple of elements $\vec{t} \in \mathfrak{g}$ obeying $\left[\boldsymbol{t}_{1}, \boldsymbol{t}_{2}\right]=\boldsymbol{t}_{3}$, and cyclic permutations. Then the solution is

$$
\vec{\phi}=\frac{\vec{t}}{y}
$$

Although any $\rho$ gives a solution, the case we want is that $\rho$ is a principal embedding. For $G=S U(N)$, this means that $\rho$ is an irreducible embedding of $S U(2)$ in $G$; for any $G$, it means that the raising operator $\boldsymbol{t}_{+}=\boldsymbol{t}_{1}+i \boldsymbol{t}_{2}$ is a "regular" element of the complexified Lie algebra $\mathfrak{g}_{\mathbb{C}}$ (this means that the subalgebra of $\mathfrak{g}_{\mathbb{C}}$ that commutes with $\boldsymbol{t}_{+}$has the minimum possible dimension). Then one can define a boundary condition by allowing precisely those solutions of (1.1) that can be approximated for $y \rightarrow 0$ by the model solution (1.3) with the regular Nahm pole. This has an analog for any $t$; the starting point, as explained in an appendix, is to set the tangential part $\vec{A}$ of the gauge field to be a specific multiple of $\vec{\phi}$, so that the equations reduce again to Nahm's equations.

When a link $L \subset W$ is included, this boundary condition is modified along $L$. A link is simply the union $L=\cup_{i} K_{i}$ of disjoint embedded circles $K_{i}$. The $K_{i}$ are labeled by representations $R_{i}^{\vee}$ of the Langlands or Goddard-NuytsOlive (GNO) dual group $G^{\vee}$ to $G$, and in this description the knots enter the formalism only via the way they enter the boundary conditions. Roughly speaking, the modification is made by requiring the presence of singular BPS monopoles supported along the $K_{i}$ with magnetic charges given by the $R_{i}^{\vee}$.

The $G$-bundle $E \rightarrow M_{4}$ has an instanton number $P$ defined in the usual way as a multiple of $\int_{M_{4}} \operatorname{Tr} F \wedge F$. (The definition of $P$ as a topological invariant involves some subtleties that are described in [14]; roughly speaking, the boundary conditions at the finite and infinite ends of $\mathbb{R}_{+}$give suitable trivializations of $E$, enabling one to define the instanton number. ${ }^{2}$ ) For each value $n$ of the instanton number, one defines an integer $a_{n}$ by "counting" (with signs that are determined by the sign of the fermion determinant of $\mathcal{N}=4$ super Yang-Mills theory) the number of solutions of the supersymmetric equations (1.1) with instanton number $n$. Then the partition function of a certain version of twisted $\mathcal{N}=4$ super Yang-Mills theory

\footnotetext{
${ }^{2}$ For general $W$ and a general choice of the boundary condition at $y=\infty, P$ takes values not in $\mathbb{Z}$ but in a certain coset of $\mathbb{Z}$ in $\mathbb{Q}$.
} 
on $M_{4}$ is

$$
Z(q)=\sum_{n} a_{n} q^{n}
$$

where the definition of $q$ in terms of parameters of $\mathcal{N}=4$ super Yang-Mills theory was explained in [14].

To get the Jones polynomial and its analogs for other groups and representations, one specializes to $W=\mathbb{R}^{3}$ and takes the boundary condition at $y=\infty$ to be simply $A, \phi \rightarrow 0$. Then for example for $G^{\vee}=S U(2)$ and $R^{\vee}$ the two-dimensional representation of $S U(2), Z(q)$ is supposed to become the Jones polynomial. Since $W=\mathbb{R}^{3}$ is the case relevant to the Jones polynomial, it will be the main example in the present paper. However, many of our considerations apply also for $W=\mathbb{R} \times C$ where $C$ is a Riemann surface, so we will consider this case as well.

A slight generalization of the above-described procedure is to modify the boundary condition at infinity so that $A$ and $\phi_{y}$ vanish but $\vec{\phi}$ approaches, up to a gauge transformation, a specified triple $\overrightarrow{\boldsymbol{a}}$ of elements of $\mathfrak{t}$, the Lie algebra of a maximal torus $T$ of $G$. Physically, this means that one takes the vacuum at infinity to be specified by a given point on the Coulomb branch. (In the presence of the Nahm pole boundary condition, turning on $\phi_{y}$ or the other two scalars of $\mathcal{N}=4$ super Yang-Mills theory - called $\sigma, \bar{\sigma}$ in [14] - would break supersymmetry; so $\vec{a}$ are the only useful Coulomb branch parameters.) Continuously turning on Coulomb branch parameters should not affect the counting of solutions of an elliptic equation, so this procedure should give a slightly more general way to compute the Jones polynomial. To describe the basic solution of equations (1.1) with a specified choice of $\vec{a}$ at infinity, one looks for a solution that still has $A=\phi_{y}=0$ and is still invariant under translations along $\mathbb{R}^{3}$, but now obeys $\lim _{y \rightarrow \infty} \vec{\phi}=g \overrightarrow{\boldsymbol{a}} g^{-1}$, for some $g \in G$. The equations still reduce to Nahm's equations (1.3). A general theorem [16] says that for any simple Lie group $G$, and any specified choice of $\overrightarrow{\boldsymbol{a}}$, there is a unique solution of Nahm's equations with a regular Nahm pole at $y=0$ and the required behavior for $y \rightarrow \infty$. This solution describes the ground state at the given point on the Coulomb branch in the absence of any 't Hooft operators on the boundary.

\subsubsection{Lift to Khovanov homology}

Although our main focus will be to recover the Jones polynomial from this framework, we will also briefly sketch how Khovanov homology is supposed to arise. A primary purpose of this is to explain the extent to which the 
“ATMP-16-3-A5-GAI" — 2013/2/1 — 19:36 — page 944 — \#10

particular values $t= \pm 1$ are or are not special, since this will be important later.

To get Khovanov homology instead of the Jones polynomial, we are supposed to "categorify" the above-described situation, which is just a fancy way to say that we must obtain everything that has been described so far from a theory in one dimension higher. For this, let $x^{1}, x^{2}, x^{3}$ be local coordinates on $W$ and decompose $\phi$ as $\phi=\vec{\phi} \cdot d \vec{x}+\phi_{y} d y$, where $\phi_{y}$ is the component of $\phi$ in the $y$ direction. Categorification is accomplished by introducing a new time coordinate $x^{0}$ and replacing $\phi_{y}$ by the covariant derivative $D / D x^{0}$. This replacement makes sense in that, since $\phi_{y}$ only appears in (1.1) inside commutators and covariant derivatives, the replacement does give a differential equation (rather than a differential operator), now on the five-manifold $\mathbb{R} \times W \times \mathbb{R}_{+}$. Moreover this differential equation, whose details are described in Section 5 of [14], is elliptic so problems of counting its solutions make sense. ${ }^{3}$

This five-dimensional lift of the four-dimensional equations (1.1) also has a surprising four-dimensional symmetry, provided we set $t= \pm 1$. The original four-dimensional symmetry relating the different directions in $M_{4}=$ $W \times \mathbb{R}_{+}$has been spoiled by the replacement $\phi_{y} \rightarrow D / D x^{0}$. But at $t= \pm 1$, the five-dimensional equations acquire a new four-dimensional symmetry: one can replace $\mathbb{R} \times W$ by a general oriented Riemannian four-manifold $M$, without additional structure, and formulate these equations on $M \times \mathbb{R}_{+}$. For studying the Jones polynomial, the values $t= \pm 1$ are not particularly distinguished; the counting of solutions of the elliptic equations (1.1) is independent of $t$. Moreover, categorification - the substitution $\phi_{y} \rightarrow D / D x^{0}-$ is not limited to $t= \pm 1$. What is special about $t= \pm 1$ is the four-dimensional symmetry of the categorified theory, which is likely to have important implications for Khovanov homology and its analogs on other manifolds.

From a physical point of view, the five-dimensional lift of equations (1.1) are BPS conditions of a certain twisted version of five-dimensional super Yang-Mills theory, formulated on $\mathbb{R} \times W \times \mathbb{R}_{+}$; they describe configurations that are invariant under one of the supercharges, which we will call $Q$. This operator obeys $Q^{2}=0$, and the space of supersymmetric ground states is the same as the cohomology of $Q$. This is the candidate for Khovanov homology. Mathematically, the five-dimensional equations can be interpreted as Morse theory flow equations, and the space of supersymmetric ground states is the analog of Floer homology for this situation. Physically, to construct the space of supersymmetric ground states, one starts with time-independent solutions of the five-dimensional equations - these are simply the solutions

\footnotetext{
${ }^{3}$ This equation has also been formulated and some basic properties described in [17].
} 
"ATMP-16-3-A5-GAI" — 2013/2/1 — 19:36 — page 945 — \#11

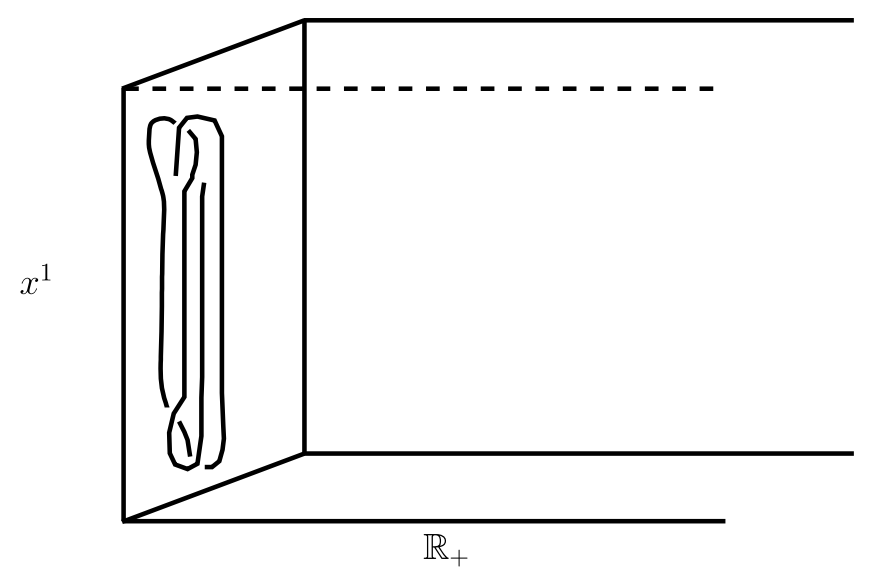

Figure 2: Stretching a knot in one direction - here taken to be the $x^{1}$ direction - to reduce to a situation that almost everywhere is nearly independent of one coordinate. After much stretching, the knot is everywhere nearly independent of $x^{1}$, except near the finite set of critical values of $x^{1}$ at which a pair of strands appears or disappears. (In the figure, these occur only at the top and bottom.)

of the original uncategorified equations (1.1) in four dimensions. Expanding around any one of these solutions, one can construct an approximate supersymmetric state, and these furnish a basis for the space of supersymmetric states in the classical approximation. Then one computes quantum corrections by taking account of tunneling between classical vacua; the tunneling events are solutions of the full five-dimensional equations.

From this point of view, the link between Khovanov homology and the Jones polynomial comes from the fact that the classical solutions that give a basis for the classical approximation to Khovanov homology are the same ones that must be counted to compute the Jones polynomial.

\subsection{Methods used in this paper}

A priori, to count the solutions of the nonlinear PDEs (1.1) is a daunting problem. Our attempts to simplify this problem are based on three ideas.

The first is a standard idea in topological field theory. We consider knots in $W=\mathbb{R} \times C$, where $C$ (which may be simply $\mathbb{R}^{2}$ ) is a two-manifold, and we parameterize $\mathbb{R}$ by $x^{1}$. We stretch our knots in the $x^{1}$ direction, so that except at a few exceptional values of $x^{1}$ where the number of strands changes, the boundary conditions are nearly independent of $x^{1}$ (figure 2). 


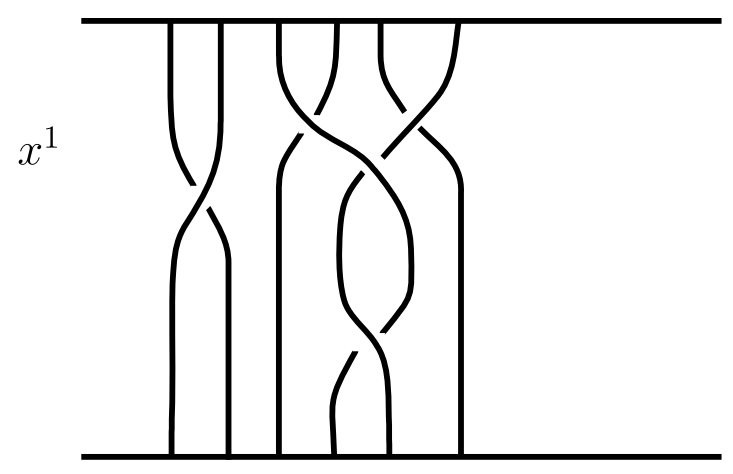

Figure 3: A braid in $I \times C$; by gluing together the top and bottom, one can make a closed braid in $S^{1} \times C$. After much stretching, a braid can be described by adiabatic evolution in $x^{1}$, with no exceptional values where this description breaks down.

We hope that, away from the exceptional values of $x^{1}$, the solutions can be approximated by solutions that are independent of $x^{1}$. Once one drops $x^{1}$, the equations reduce to equations in three dimensions. The reduced equations preserve more supersymmetry and one may hope to understand their solutions.

After finding the three-dimensional solutions, to recover a fourdimensional picture, we have to take into account an adiabatic variation of the parameters in the three-dimensional equations. This is because our knot, even after stretching, is not quite independent of $x^{1}$. We also have to consider the jumping that occurs when the number of strands changes. Actually, there is an important special case in which one only has to consider the adiabatic variation of parameters. This is the case (figure 3 ) that $\mathbb{R} \times C$ is replaced by $S^{1} \times C$ (or $I \times C$ where $I$ is a closed interval, although this introduces questions about boundary conditions) and the link is replaced by a braid. In this situation, one would study not the Jones polynomial but its associated braid group representations, which are also of great interest.

One important thing to mention about this program is that it is not guaranteed to work. As one stretches a knot in the $x^{1}$ direction, the solution might simultaneously "spread" in the $y$-direction (figure 4) so that even after stretching, the solution might not approach an $x^{1}$-independent limit. In fact, we will find that this happens under some conditions. One of our main tasks will be to understand conditions under which the sort of behavior suggested in figure 4 does not occur.

In carrying out the program that we have just described, we start in Section 2 at $t= \pm 1$ because these are special values for Khovanov homology (as 


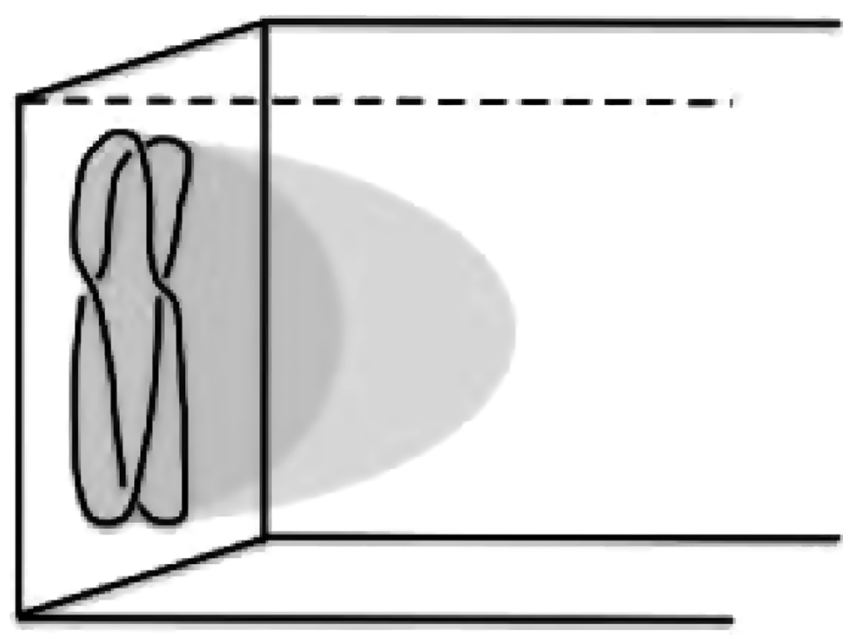

Figure 4: As a knot is stretched along the boundary, a solution of the supersymmetric equations might become delocalized in the $y$-direction, normal to the boundary. This is schematically indicated here; the shaded region indicates the spatial extent of a solution - that is, of the region over which the chosen solution deviates significantly from the one that describes the vacuum in the absence of knots - and its thickness is proportional to the amount that the knot has been stretched.

we recalled in Section 1.1.1 above) and also because some simplifications in the three-dimensional equations at $t= \pm 1$ were already found in Section 3.6 of [14].

Because we encounter some puzzling phenomena (which we will ultimately understand along the lines of figure 4), we look for some additional simplifications. In doing so, we primarily exploit two ideas.

The first idea is to modify the boundary conditions to incorporate gauge symmetry breaking. The basic idea was already explained at the end of Section 1.1: instead of asking for $\vec{\phi}$ to vanish at infinity, we ask for $\vec{\phi} \rightarrow$ $g \overrightarrow{\boldsymbol{a}} g^{-1}$, where $g \in G$ and the three components of $\overrightarrow{\boldsymbol{a}}=\left(\boldsymbol{a}_{1}, \boldsymbol{a}_{2}, \boldsymbol{a}_{3}\right)$ take values in a Cartan subalgebra of the Lie algebra $\mathfrak{g}$ of $G$. (In the more general case $W=\mathbb{R} \times C$, we would similarly modify the boundary condition to require that the component of $\phi$ in the $\mathbb{R}$ direction is in a specified conjugacy class at infinity.) Continuously changing the boundary conditions in this way should not change the counting of solutions that leads to the Jones polynomial. On the other hand, in such counting problems one often finds that perturbing to a more generic situation can make things easier. Moreover, in the present case, taking $\overrightarrow{\boldsymbol{a}}$ to be generic reduces the nonabelian gauge theory that we are studying to an abelian theory at low energies. If we scale up our knots 
"ATMP-16-3-A5-GAI" — 2013/2/1 — 19:36 — page 948 — \#14

so that all relevant directions of the $K_{i}$ are large compared to $1 /|\overrightarrow{\boldsymbol{a}}|$, then we can reasonably hope to find some sort of effective abelian description of the relevant phenomena.

The second idea that we exploit is perhaps even more obvious. Since the equations that arise at $t=1$ with the Nahm pole boundary conditions described above are rather special, we perturb the value of $t$ and/or the Nahm pole boundary conditions to something more generic. This proves to be very fruitful, especially when combined with gauge symmetry breaking.

\subsection{Outline and results}

In Section 2, we analyze the three-dimensional reduction of equations (1.1) at $t=1$. We get an interesting description in terms of Higgs bundles with some additional structure, but it becomes clear that the program suggested in figure 2 will encounter some difficulties at $t=1$. In Section 3 , we perturb the equations to $t \neq 1$ and find that this offers a much more promising framework for understanding the Jones polynomial. The equations for generic $t$ have surprising and useful relations to a variety of topics in mathematical physics, including the Bethe equations for an integrable spin system known as the Gaudin model, and certain special "degenerate" conformal blocks of the Virasoro algebra; the rest of the paper is based on these relations. In Section 4, we discuss the general framework for constructing braid group representations from adiabatic evolution of the parameters governing timeindependent solutions. The general framework is a little abstract, but in Section 5, we show that in our particular problem, it can be made very concrete using the free-field representation of certain Virasoro conformal blocks. In Section 6, we implement that idea in detail. This finally enables us to understand how the Jones polynomial and the braid group representations associated to it can be recovered by counting solutions of the four-dimensional BPS equations (1.1). Section 7 is devoted to describing an effective superpotential for BPS monopoles that can be used to understand some of the subtle results of Sections 2 and 3. In Section 8, we place some structures encountered in this paper in a wider context of mathematical physics. Three appendices fill in details of the derivations.

\section{Analysis at $t=1$}

\subsection{Some preliminaries}

As explained in the introduction, after stretching a knot along the first factor of $M_{4}=\mathbb{R} \times C \times \mathbb{R}_{+}$, we want to find the solutions that are independent of 


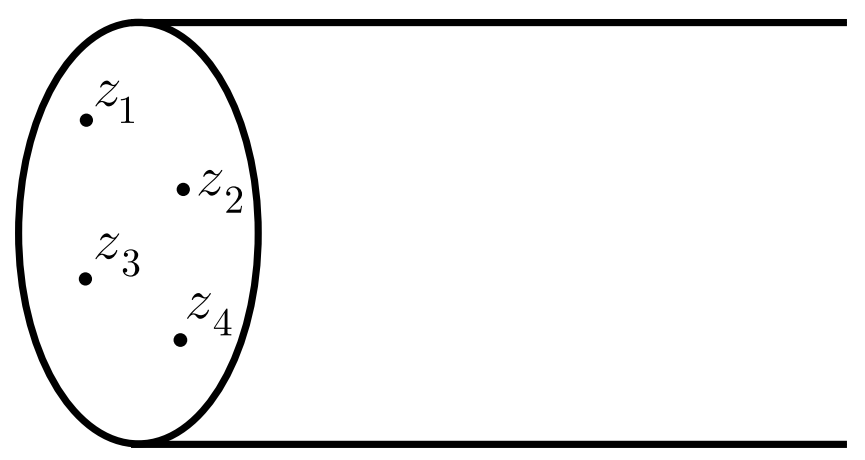

Figure 5: In a time-independent situation, we look for solutions on a threemanifold $M_{3}=C \times \mathbb{R}_{+}$, where $C$ (taken here to be a two-sphere) is a Riemann surface. Knots are placed at points on the boundary of $M_{3}$, labeled here as $z_{1}, \ldots, z_{4}$.

the first coordinate (which we call $x^{1}$ ), that is the solutions that obey reduced equations on the three-manifold ${ }^{4} \quad M_{3}=C \times \mathbb{R}_{+}$. Here $C$ is a Riemann surface (which may be simply $\mathbb{R}^{2}$ ), and $\mathbb{R}_{+}$is the half-line $y \geq 0$. If knots are present, we take their support to be of the time-independent form $\mathbb{R} \times$ $z_{i} \times\{0\}$, where the $z_{i}$ are points in $C$, and $\{0\}$ is the endpoint $y=0$ of $\mathbb{R}_{+}$. The picture is sketched in figure 5 .

Solutions that can be derived from three dimensions preserve more than the generic amount of supersymmetry - they preserve four supercharges, to be precise - and accordingly, as described in Section 3.6 of [14], their structure simplifies. In all cases that we will encounter in the present paper, the reduced equations can be usefully described in terms of three differential operators $\mathcal{D}_{i}, i=1,2,3$. The equations say that the $\mathcal{D}_{i}$ commute

$$
\left[\mathcal{D}_{i}, \mathcal{D}_{j}\right]=0
$$

and obey a moment map condition

$$
\sum_{i=1}^{3}\left[\mathcal{D}_{i}, \mathcal{D}_{i}^{\dagger}\right]=0
$$

where $\mathcal{D}_{i}^{\dagger}$ is the adjoint of $\mathcal{D}_{i}$ in a natural sense. The commutativity constraint (2.1) is invariant under complex-valued gauge transformations

\footnotetext{
${ }^{4}$ To minimize confusion, we note the following. In this paper, we use two different decompositions of $M_{4}=\mathbb{R} \times C \times \mathbb{R}_{+}$as the product of a three-manifold and a one-manifold: we write $M_{4}=W \times \mathbb{R}_{+}$with $W=\mathbb{R} \times C$, but also $M_{4}=\mathbb{R} \times M_{3}$ with $M_{3}=C \times \mathbb{R}_{+}$.
} 


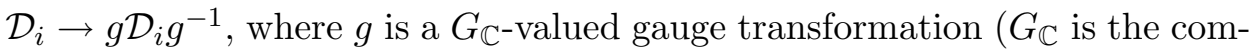
plexification of $G$ ), while the moment map condition (2.2) is only invariant under $G$-valued gauge transformations. What will make our problem tractable is that solutions of the combined system of equations modulo $G$ valued gauge transformations are equivalent to solutions of just the commutativity constraint (2.1) modulo $G_{\mathbb{C} \text {-valued gauge transformations. But }}$ solutions of the commutativity constraint modulo complex gauge transformations can be described in terms of holomorphic quantities, so it is possible to understand them.

Different instances of this structure vary by the construction of the $\mathcal{D}_{i}$, which depends on the choice of $t$, and also on the boundary conditions that we assume at $y=0$ and at $y=\infty$. The most basic case considered in [14] is that $t=1$ (or -1 ) and the boundary condition is given by a Nahm pole in the part of $\phi$ tangential to the boundary. That boundary condition sets to zero $\phi_{y}$, the normal part of $\phi$, at $y=0$. If we also require $\phi_{y}$ to vanish for $y \rightarrow \infty$, then a simple vanishing argument shows that in a solution that is independent of $x^{1}, \phi_{y}$ is identically zero; similarly, $A_{1}$, the component of $A$ in the $x^{1}$ direction, vanishes in a three-dimensional solution. Once $\phi_{y}$ and $A_{1}$ are set to zero, the equations can be put in the above-described form, as shown in detail in [14], Section $3.6,{ }^{5}$ with

$$
\begin{aligned}
& \mathcal{D}_{1}=\frac{D}{D x^{2}}+\mathrm{i} \frac{D}{D x^{3}} \\
& \mathcal{D}_{2}=\left[\phi_{2}-\mathrm{i} \phi_{3}, \cdot\right] \\
& \mathcal{D}_{3}=\frac{D}{D y}-\mathrm{i}\left[\phi_{1}, \cdot\right]
\end{aligned}
$$

and the moment map condition

$$
0=\sum_{i=1}^{3}\left[\mathcal{D}_{i}, \mathcal{D}_{i}^{\dagger}\right]=F_{23}-\left[\phi_{2}, \phi_{3}\right]-D_{y} \phi_{1}
$$

In writing these formulae, we have simply taken $C=\mathbb{R}^{2}$ with coordinates $x^{2}, x^{3}$. However, it is helpful to introduce a complex coordinate $z=x^{2}+$ $\mathrm{i} x^{3}$, and to write $\phi_{2} d x^{2}+\phi_{3} d x^{3}=\varphi d z+\bar{\varphi} d \bar{z}$; also we introduce a complex connection $\mathcal{A}_{y}=A_{y}-\mathrm{i} \phi_{1}$ for parallel transport in the $y$ direction and write

\footnotetext{
${ }^{5}$ Our notation differs from the notation used there by a relabeling of the coordinates $x^{i} \rightarrow x^{i+1}$ (whose purpose is to make "room" for a new time coordinate $x^{0}$ upon categorification). Also, for later convenience we permute the $\mathcal{D}_{i}$ in an obvious way.
} 
$\mathcal{D}_{y}=d_{y}+\left[\mathcal{A}_{y}, \cdot\right]$. Then we can write

$$
\begin{aligned}
& \mathcal{D}_{1}=2 \frac{D}{D \bar{z}}, \\
& \mathcal{D}_{2}=2[\varphi, \cdot], \\
& \mathcal{D}_{3}=\frac{\mathcal{D}}{\mathcal{D} y} .
\end{aligned}
$$

With this way of writing the $\mathcal{D}_{i}$, they make sense on an arbitrary Riemann surface $C$, with $D / D \bar{z}$ understood as the $\bar{\partial}$ operator and $\varphi$ as a $(1,0)$-form on $C$.

As one would expect in a geometry that preserves four supercharges, the commutativity constraint $\left[\mathcal{D}_{i}, \mathcal{D}_{j}\right]=0$ can be derived from a superpotential, namely

$$
\mathcal{W}=\frac{1}{4 \pi i} \int_{C \times \mathbb{R}_{+}} \operatorname{Tr} \varphi \mathcal{F}_{y \bar{z}},
$$

with $\mathcal{F}_{y \bar{z}}=\left[\mathcal{D}_{y}, D_{\bar{z}}\right]$. To be more exact, in varying $\mathcal{W}$ to derive the conditions $\left[\mathcal{D}_{i}, \mathcal{D}_{j}\right]=0$, one should require the variation of $A$ at $y=0$ to vanish. Otherwise, the variation of $\mathcal{W}$ contains additional delta function terms at $y=0$.

The equations $\left[\mathcal{D}_{i}, \mathcal{D}_{j}\right]=\sum_{i}\left[\mathcal{D}_{i}, \mathcal{D}_{i}^{\dagger}\right]=0$ have been called the extended Bogomolny equations in [15]; actually, these equations are a hybrid of the equations of Nahm, Hitchin, and Bogomolny. They reduce to Nahm's equations if we drop the dependence on $z$, to Hitchin's equations if we drop the dependence on $y$, and to the Bogomolny equations if we set $\varphi=0$. This is not just an analogy: we can borrow standard strategies from the theory of moduli spaces of Nahm, Hitchin, or Bogomolny equations.

The equation $\left[\mathcal{D}_{1}, \mathcal{D}_{2}\right]=0$, taken for fixed $y$, defines a Higgs bundle $(E, \varphi)$ in the sense of Hitchin. The fact that $\mathcal{D}_{1}$ and $\mathcal{D}_{2}$ commute with $\mathcal{D}_{3}$ simply means that the Higgs bundle is independent of $y$, up to a complex-valued gauge transformation. When we specialize to $C=\mathbb{R}^{2} \cong \mathbb{C}$, we get a Higgs bundle on $\mathbb{C}$ that can be understood as a Higgs bundle on $\mathbb{C P}^{1}=\mathbb{C} \cup \infty$, possibly with a singularity at infinity. This case will be considered in Section 2.5. There is another natural way for singularities of the Higgs bundle to arise. As in Section 6 of [14], one may include surface defects supported on codimension two submanifolds in $M_{4}$. Taking these to be of the form $\mathbb{R} \times q_{i} \times \mathbb{R}_{+}$, where the $q_{i}$ are points in $C$, the construction summarized above still applies and the Higgs bundles acquire singularities at the points $q_{i}$. We consider this situation in Section 8.4. 
Additional structure arises from boundary conditions at $y=0$ and $y=\infty$. We will discuss the consequences of the Nahm pole at $y=0$ in Section 2.2. The analogy to Nahm's equations will be useful: we can extract some holomorphic data from the commuting pair $\mathcal{D}_{2}, \mathcal{D}_{3}$ for any given point in $C$. As $\mathcal{D}_{2}, \mathcal{D}_{3}$ commute with $\mathcal{D}_{1}$, this data varies holomorphically on $C$, or possibly meromorphically in the presence of singularities.

Finally, an analogy with the Bogomolny equations will help us understand the physical content of our solutions, especially when we turn on gauge symmetry breaking for $y \rightarrow \infty$. Indeed, the holomorphic data in the commuting pair $\mathcal{D}_{1}, \mathcal{D}_{3}$ is analogous to the data which specifies the position of BPS monopoles in a solution of the Bogomolny equations.

\subsection{The boundary condition}

Our next task is to analyze the boundary conditions, first in the absence of singular monopoles. The model solution (1.3) at $t=1$ has a singularity in $\mathcal{D}_{2}$ and $\mathcal{D}_{3}$, but not in $\mathcal{D}_{1}$, simply because $\mathcal{D}_{1}$ does not contain the scalar fields. For $\mathfrak{g}=\mathfrak{s u}(2)$, with a standard choice of the Lie algebra elements $\boldsymbol{t}$, this solution is explicitly

$$
\varphi=\frac{1}{y}\left(\begin{array}{ll}
0 & 1 \\
0 & 0
\end{array}\right), \quad A_{\bar{z}}=0, \quad \mathcal{A}_{y}=\frac{1}{2 y}\left(\begin{array}{cc}
1 & 0 \\
0 & -1
\end{array}\right)
$$

We consider the matrices here to act on the fiber of a trivial rank two complex vector bundle $E \rightarrow \mathbb{R}^{2} \times \mathbb{R}_{+}$. For $G=S O(3)$, on a general Riemann surface, there could be a global obstruction to defining $E$ as a rank two bundle and one would then consider instead the corresponding adjoint bundle $\operatorname{ad}(E)$. Because our considerations will be local along the Riemann surface $C$, a reformulation in terms of the adjoint representation does not change much, and we will omit this.

The solution (2.7) can be written

$$
\varphi=g \varphi_{1} g^{-1}, \quad \mathcal{D}_{y}=g \frac{d}{d y} g^{-1}
$$

with

$$
\varphi_{1}=\left(\begin{array}{ll}
0 & 1 \\
0 & 0
\end{array}\right)
$$


and $g$ a $G_{\mathbb{C}^{-v a l u e d ~ g a u g e ~ t r a n s f o r m a t i o n ~ t h a t ~ i s ~ s i n g u l a r ~ a t ~} y=0}$

$$
g=\left(\begin{array}{cc}
y^{-1 / 2} & 0 \\
0 & y^{1 / 2}
\end{array}\right)
$$

In other words, the solution is obtained by the complex-valued gauge transformation $g$ from a trivial solution $\varphi=\varphi_{1}, A_{\bar{z}}=\mathcal{A}_{y}=0$.

We want to consider solutions that look like the model solution near $y=0$, up to a gauge transformation. We would like to express this constraint in terms of the Higgs bundle data $(E, \varphi)$ away from the boundary. For that purpose, it is useful to consider the behavior of a local holomorphic section $s$ of the gauge bundle $E$ that is invariant under parallel transport in the $y$-direction. We will first do the calculation very explicitly for the model solution, and then identify which features are valid more generally.

Let $s$ be a section of the gauge bundle $E$ that obeys

$$
\frac{\mathcal{D} s}{\mathcal{D} y}=0 .
$$

Since $\mathcal{D} / \mathcal{D} y=g(d / d y) g^{-1}$, the solutions of this equation are of the form $s=g s_{0}$, where $s_{0}$ is independent of $y$. Thus a general solution takes the form

$$
s=\left(\begin{array}{c}
a y^{-1 / 2} \\
b y^{1 / 2}
\end{array}\right)
$$

with constants $a, b$. In particular, a generic vector in $E$, when parallel transported in the $y$-direction to $y=0$, will blow up as $y^{-1 / 2}$. There is a one-dimensional subspace consisting of solutions of $\mathcal{D} s / \mathcal{D} y=0$ that actually vanish as $y^{1 / 2}$ for $y \rightarrow 0$. This subspace is simply characterized by the condition $a=0$.

We write $E_{y}$ for the restriction of $E \rightarrow C \times \mathbb{R}_{+}$to $C \times\{y\}$ for any fixed $y>0$. Parallel transport using $\mathcal{D}_{y}$ gives a natural identification of the $E_{y}$ for all $y$, and we write simply $E$ for $E_{y}$, regarded as a bundle over $C$. Similarly, the restriction of $\varphi$ to $C \times\{y\}$ is independent of $y$, up to parallel transport by $\mathcal{D}_{y}$. So by restriction to $C \times\{y\}$, we get a Higgs bundle $(E, \varphi) \rightarrow C$.

The "small" sections of $E$ - the solutions $s$ of $\mathcal{D} s / \mathcal{D} y=0$ that vanish for $y \rightarrow 0-$ generate a rank one sub-bundle $L \subset E$. In the model solution, it is simply the sub-bundle of sections of $E$ of the form $\left(\begin{array}{l}0 \\ b\end{array}\right) . L$ is a holomorphic sub-bundle of $E \rightarrow C$; a section of $L$ is holomorphic if it is annihilated by 
$\mathcal{D}_{1}=2 D / D \bar{z}$. Concretely, in the model solution, a section $\left(\begin{array}{l}0 \\ b\end{array}\right)$ of $L$ is holomorphic if $b$ is a holomorphic function of $z$.

The fundamental reason that $L$ is holomorphic is that, as $\left[\mathcal{D}_{2}, \mathcal{D}_{3}\right]=0$, we can ask for a small solution of $\mathcal{D}_{3} s=0$ to also be annihilated by $\mathcal{D}_{2}=$ $2 D / D \bar{z}$. However, we cannot also ask for a small solution to be annihilated by $\varphi=\mathcal{D}_{1} / 2$. This is clear from the above formulae; in the model solution, $\varphi$ annihilates a vector if the bottom component vanishes, not if the top component vanishes.

To measure the failure of $L$ to be $\varphi$-invariant, we can proceed as follows. If $L$ is a trivial line bundle, which will be the case in our applications, then we can pick a section $s$ of $L$ that is everywhere nonzero. For our model solution, we just pick $s=\left(\begin{array}{l}0 \\ 1\end{array}\right)$. Then we define

$$
\kappa=s \wedge \varphi s
$$

For the model solution, we see that $\kappa=1$, and in particular $\kappa$ is everywhere nonzero. Nonvanishing of $\kappa$ means that $\varphi s$ is not a multiple of $s$, so $L$ is not invariant under multiplication by $\varphi$. (If $\mathbb{R}^{2}$ is replaced by a general Riemann surface $C$, it might be impossible to pick an $s$ that is globally nonzero, but one can still pick a local section $s$ of $L$ and measure the failure of $L$ to be $\varphi$-invariant by computing $\kappa=s \wedge \varphi s$. Whether $\kappa$ vanishes does not depend on the choice of $s$ as long as it is nonzero.)

Three basic properties of $s$ which held for the model solution remain true for any solution with a regular Nahm pole, since they are unaffected by the subleading behavior of $\mathcal{D}_{y}$ and $\varphi$ as $y \rightarrow 0$ :

- A generic vector in $E$, when parallel transported in the $y$-direction to $y=0$, will blow up as $y^{-1 / 2}$.

- The sections $s$ that under parallel transport to $y=0$ actually vanish span a rank 1 holomorphic sub-bundle $L \subset E$.

- Finally, we cannot also ask for a small section to be annihilated by $\varphi=\mathcal{D}_{1} / 2$. On the contrary, in a solution that can be approximated by the model solution near $y=0, \varphi$ does not annihilate a small section at any point in $C$.

Consequently, a solution of the full equations with a regular Nahm pole for $y \rightarrow 0$ gives not just a Higgs bundle $(E, \varphi)$. Rather, there is some additional structure: $E$ is endowed with a holomorphic line sub-bundle $L$, which is nowhere stabilized by $\varphi$. On $\mathbb{R}^{2}, L$ is inevitably trivial, so we can simply say 
that an everywhere nonzero holomorphic section $s$ of $E$ exists with $s \wedge \varphi s=$ 1. Note that this condition is far from sufficient to determine $\varphi$. If $s=\left(\begin{array}{l}0 \\ 1\end{array}\right)$, then the condition $s \wedge \varphi s=1$ fixes the upper right matrix element of $\varphi$ and puts no condition on the others. The reason that only one matrix element of $\varphi$ is fixed in terms of $s$ is that when we make a gauge transformation that behaves like (2.10) for $y \rightarrow 0, \varphi$ acquires a singularity that only depends on its upper right matrix element. In an appropriate situation (on a Riemann surface $C$ of higher genus, or on $\mathbb{R}^{2}$ in the presence of singular monopoles, as introduced shortly), there can be a nontrivial moduli space of triples $(E, \varphi, L)$ with $E$ and $L$ fixed and only $\varphi$ varying. This is explained in Section 8 .

For any triple $(E, \varphi, L)$, we expect that the full system of equations can be solved by a complex gauge transformation. Suppose we are given a $y$-independent Higgs bundle $(E, \varphi)$, and a holomorphic sub-bundle $L \subset E$ which is nowhere stabilized by $\varphi$. In a basis of $E$ given by $\varphi s$ and $s$, we make the gauge transformation (2.10). This will reproduce the Nahm pole singularity at $y=0$, but generically it will not give a solution of the moment map condition (2.4). By further making a smooth complex gauge transformation, one can hope to get a solution of the moment map condition.

\subsubsection{Adding singular monopoles}

In order to add singular monopoles at the boundary, we need to replace the Nahm model solution with a more general singular solution, given in [14], Section 3.6. Let us consider the case of a single singular monopole, located at $y=z=0$. The model solution has the same singularity as before for $y \rightarrow 0$ at $z \neq 0$, but has a more complicated form near $y=z=0$. It can be obtained by a complex-valued gauge transformation, described explicitly in [14], from a solution of the commutativity constraint with

$$
\varphi=\left(\begin{array}{cc}
0 & z^{k} \\
0 & 0
\end{array}\right), \quad A_{z}=\mathcal{A}_{y}=0
$$

To express this in our present language, $L$ is still spanned by sections that in the gauge (2.14) are multiples of $s=\left(\begin{array}{l}0 \\ 1\end{array}\right)$; this is because $g$ has similar behavior as before for $y \rightarrow 0$. We now have

$$
s \wedge \varphi s=z^{k} .
$$

The zero of $s \wedge \varphi s$ is interpreted as the position of the singular monopole, and its degree is the charge. This interpretation suggests immediately what 
a solution with several singular monopoles should mean. We consider a solution described by a Higgs bundle $(E, \varphi)$ with a sub-bundle $L \subset E$ that is generically not $\varphi$-invariant. If

$$
s \wedge \varphi s=\prod_{a=1}^{s}\left(z-z_{a}\right)^{k_{a}}
$$

then we say that the solution has singular monopoles of charges $k_{a}$ at the locations $z_{a}$. One hopes to be able to prove that given such data, there is a unique solution whose singularity near each $z=z_{a}$ agrees with that of the singular model solution.

Though we mainly consider $\mathfrak{s u}(2)$ in the present paper, we can readily generalize these statements to a more general Lie algebra. For simplicity, take $G=S U(N)$ and view $E$ as a complex vector bundle of rank $N$. Consider a Nahm pole based on the principal embedding of $\mathfrak{s u}(2) \rightarrow \mathfrak{s u}(N)$. The eigenspaces of $\boldsymbol{t}_{3}$ (in the fundamental $N$-dimensional representation of $\mathfrak{s u}(N))$ are one-dimensional, and we have a line bundle $L$ defined by sections, which decrease as fast as possible as $y \rightarrow 0$. The only constraint on $L$ is that, away from the positions of singular monopoles,

$$
L \oplus \varphi L \oplus \cdots \oplus \varphi^{N-1} L=E .
$$

When we specialize to $C=\mathbb{R}^{2} \cong \mathbb{C}$, we can define the line sub-bundle $L$ by a specific section $s$ of $E$, defined up to rescaling, such that the sections $s, \varphi s, \ldots, \varphi^{N-1} s$ are linearly independent. This constraint is relaxed at the location of singular monopoles, in a way which depends on their charges. An equivalent description in terms of zeroes of matrix elements of $\varphi$ is given in [14], equation (3.59).

This can be extended to the case of a general Nahm pole at $y=0$, not necessarily associated to a principal embedding of $\mathfrak{s u}(2)$. (This extension will not be studied in the present paper.) In general, for $y \rightarrow 0$, there are local sections growing as either integer or half-integer powers of $y$. We can define a flag of holomorphic sub-bundles $E_{n}$ of $E$ by looking at local sections, which grow at most as a given power $y^{(n-N) / 2}$ as $y \rightarrow 0$. Clearly, $E_{n+1} \subset E_{n}$; also $E_{0}=E$, and $E_{n}=0$ for large enough $n$. Upon rescaling by $y^{-(n-N) / 2}$, a generic vector in $E_{n}$ has a finite limit as $y \rightarrow 0$, and this limit is an eigenvector of $\boldsymbol{t}_{3}$ with eigenvalue $(N-n) / 2$. The kernel of this map is $E_{n+1}$; hence the $y \rightarrow 0$ limit identifies the quotient spaces $E_{n} / E_{n+1}$ with the eigenspaces of $\boldsymbol{t}_{3}$. Multiplication by $\varphi$ gives maps $E_{n} \rightarrow E_{n-2}$. As $\varphi \sim \frac{\boldsymbol{t}_{+}}{y}$, the holomorphic maps $\phi_{n}: E_{n} / E_{n+1} \rightarrow E_{n-2} / E_{n-1}$ can be identified with the action of $\boldsymbol{t}_{+}$on the eigenspaces of $\boldsymbol{t}_{3}$. 


\subsection{Solutions without symmetry breaking}

Now we want to use the ideas that have just been described to determine some moduli spaces of solutions of the supersymmetric equations (2.1) and (2.2). We will do this for $G=S O(3)$, so that the dual group whose representations label the singular monopoles is $G^{\vee}=S U(2)$.

First, we work at the origin of the Coulomb branch; this means that we consider solutions such that the scalar fields $\vec{\phi}$ vanish for $y \rightarrow \infty$. In particular, $\varphi$ must vanish at infinity. Since the equation $\mathcal{D}_{y} \varphi=0$ means that the conjugacy class of $\varphi$ is $y$-independent, $\varphi$ can only vanish at infinity if it is everywhere nilpotent. This means that by a complex gauge transformation, we can make $\varphi$ upper triangular:

$$
\varphi=\left(\begin{array}{cc}
0 & p(z) \\
0 & 0
\end{array}\right)
$$

with some polynomial $p(z)$. We cannot, however, put $s$ in a standard form at the same time. So we simply take

$$
s=\left(\begin{array}{l}
P(z) \\
Q(z)
\end{array}\right),
$$

with polynomials $P, Q$. Without changing $\varphi$, we can make an upper triangular gauge transformation, shifting $P$ by a polynomial multiple of $Q$ :

$$
P(z) \rightarrow P(z)+U(z) Q(z) .
$$

This is the only freedom, apart from a rescaling of $s$ by a complex constant.

Now let us ask how we can pick $p, Q$, and $P$ to describe a configuration with singular monopoles of charges $k_{a}$ at the points $z_{a}$ in the boundary. Since $s \wedge \varphi s=p Q^{2}$, the condition that we need is

$$
p(z) Q(z)^{2}=\prod_{a}\left(z-z_{a}\right)^{k_{a}}:=K(z) .
$$

If the singular monopoles all have minimal charge, $k_{a}=1$, we can only obey this with $p=K$ and $Q= \pm 1$. Then we can set $P(z)=0$ by a transformation (2.20), and changing the sign of $Q$ multiplies $s$ by an inessential constant. So at the origin of the Coulomb branch, there is a unique solution with singular monopoles of specified locations and minimal magnetic charge. 
“ATMP-16-3-A5-GAI" — 2013/2/1 — 19:36 — page 958 — \#24

This fact is actually an obstruction to the program that was described in Section 1.2 (see figure 2). The case of singular monopoles of minimal charge is supposed to be dual to the Jones polynomial, which is the invariant computed in Chern-Simons gauge theory for $G^{\vee}=S U(2)$ with all knots labeled by the two-dimensional representation of $S U(2)$. If there is only one solution, this means that the physical Hilbert space associated to this problem is one-dimensional. The Jones representations of the braid group would then be of rank 1 . This is certainly not the case. We must be running into some version of the problem that was indicated in figure 4 . We will get a clearer picture of what is happening after including symmetry breaking in Section 2.4, and after deforming to $t \neq 1$ in Section 3. This will ultimately enable us to circumvent the obstacle just described.

What happens if some singular monopoles have nonminimal charge? In this case, we encounter moduli spaces of solutions. In general, we solve (2.21) by

$$
p(z)=\prod_{a}\left(z-z_{a}\right)^{m_{a}}, \quad Q=\prod_{a}\left(z-z_{a}\right)^{r_{a}},
$$

with

$$
m_{a}+2 r_{a}=k_{a}
$$

For $Q$ as in (2.22), we cannot set $P$ to 1 by a transformation (2.20); rather, the values of $P$ and its first $r_{a}-1$ derivatives are invariants at each point $z=z_{a}$. So, there are $r_{a}$ moduli associated to each $z_{a}$, where the possible values of $r_{a}$ are $\left\{0,1, \ldots,\left[k_{a} / 2\right]\right\}$. We will argue beginning in Section 2.4 and in most detail in Section 7 that the moduli represent the positions in the $y$-direction of some smooth BPS monopoles, together with some conjugate angles.

In implementing the program described in Section 1.2, if some knots are labeled by integers $k_{a}>1$ - in other words, by representations of $G^{\vee}=$ $S U(2)$ of dimension $k_{a}+1>2$ - one would have to handle the evolution of the four-dimensional solution as a path in the moduli space of threedimensional solutions. (This would be done by quantizing the moduli space to get an appropriate space of physical states, in which the evolution would take place.) Instead of following that route, we will perturb the equations that we have just analyzed to more generic ones, with symmetry breaking at infinity or with $t \neq 1$. This will eventually reduce all of our moduli spaces to finite collections of points. 


\section{4 "Real" symmetry breaking}

Our first approach to getting a clearer understanding of the solutions and a more useful reduction to three dimensions - will be to move on the Coulomb branch. The basic idea was already explained at the end of Section 1.1. We fix some constant, nonzero expectation values $\vec{a}$ for the tangential scalar fields $\vec{\phi}$, and consider only solutions of the supersymmetric equations (1.1) such that $\lim _{y \rightarrow \infty} \vec{\phi}=g \overrightarrow{\boldsymbol{a}} g^{-1}$, for some $g \in G$.

From our present point of view, we want to analyze the reduced threedimensional equations in the context of symmetry breaking. The reduction splits off the $x^{1}$ direction, so we write $\vec{\phi} \cdot \overrightarrow{d x}=\phi_{1} d x^{1}+\varphi d z+\bar{\varphi} d \bar{z}$. The effects of an expectation value for $\phi_{1}$ or for $\varphi$ will be quite different. We first consider the case of turning on $\phi_{1}$ only. As we will see, this has the effect of making the solutions somewhat more physically transparent, without adding or removing solutions.

For $G=S O(3)$, we can pick a gauge where $\phi_{1}$ is constant at large $y$. This means that for large $y, \mathcal{A}_{y}=A_{y}-\mathrm{i} \phi_{1}$ approaches a constant matrix at infinity, say such that

$$
\mathcal{A}_{y}=\left(\begin{array}{cc}
\boldsymbol{a}_{1} & 0 \\
0 & -\boldsymbol{a}_{1}
\end{array}\right) .
$$

with $\boldsymbol{a}_{1}>0$. This expectation value breaks $S O(3)$ to $U(1)$. We pick a generator of the unbroken $U(1)$, normalized so that the off-diagonal components of an adjoint-valued field, which are the fields of minimum electric charge for $G=S O(3)$, have charges \pm 1 :

$$
\mathcal{Q}=\left(\begin{array}{cc}
-1 / 2 & 0 \\
0 & 1 / 2
\end{array}\right)
$$

The choice of sign will be convenient.

The equation $\mathcal{D}_{y} \varphi=0$ implies that the lower-triangular component of $\varphi$ must be zero, since otherwise it would grow exponentially fast at infinity. The diagonal component must also be zero, since otherwise $\varphi$ would have a nonzero limit at infinity. Hence symmetry breaking provides a natural frame in which $\varphi$ is strictly upper-triangular for $y \rightarrow \infty$, just as the boundary condition did for $y \rightarrow 0$. But $\varphi$ decays exponentially fast at large $y$.

In the low-energy effective $U(1)$ theory, a field of electric charge 1 is a section of a line bundle that we will call $\mathcal{M}$. The first Chern class of $\mathcal{M}$, integrated over the plane given by $y=y_{0}$, for some large constant $y_{0}$, is 
“ATMP-16-3-A5-GAI" — 2013/2/1 — 19:36 — page 960 — \#26

an integer that we will call the magnetic charge $\boldsymbol{m}$. The upper triangular matrix element of $\varphi$

$$
\varphi \sim\left(\begin{array}{ll}
0 & \widetilde{p} \\
0 & 0
\end{array}\right)
$$

is a section of $\mathcal{M}^{-1}$, and hence the magnetic charge, which is minus the first Chern class of $\mathcal{M}^{-1}$, is minus the number of zeroes of $\widetilde{p}$. This is the same as minus the number of zeroes of $p$ (defined in (2.18)), which is equivalent to $\widetilde{p}$ by a complex gauge transformation. So in the notation of $(2.23)$, the magnetic charge is

$$
\boldsymbol{m}=-\sum_{a} m_{a}=\sum_{a}\left(-k_{a}+2 r_{a}\right)
$$

This means that for a given configuration of singular monopoles or 't Hooft operators at $y=0$, the smallest possible value of $\boldsymbol{m}$ is $-\sum_{a} k_{a}$. Every time that we add a zero to $Q, \boldsymbol{m}$ increases by 2 .

We propose that this fact can be interpreted in terms of smooth BPS monopoles. If we simply set $\varphi=0$, the extended Bogomolny equations that we have been studying reduce to the usual Bogomolny equations. Far from the boundary, the Bogomolny equations are a very good approximation, since $\varphi$ is so small. The Bogomolny equations on $\mathbb{R}^{3}$ admit smooth monopole solutions of charge 2. (We measure magnetic charge in units such that the minimum magnetic charge allowed by Dirac quantization is \pm 1 . This is also the magnetic charge of a minimum charge singular monopole or 't Hooft operator, but smooth monopoles have even charge.) Our proposal is that if $Q$ has a zero of order $r_{a}$ at $z=z_{a}$, then there are $r_{a}$ smooth monopoles located at $z=z_{a}$. This statement has a precise meaning only if the monopoles are located at very large $y$, where the extended Bogomolny equations reduce to the ordinary ones, and have smooth monopoles as solutions. However, we have found that if $Q$ has a zero of order $r_{a}$ at $z=z_{a}$, then there are precisely $r_{a}$ complex moduli associated to the point $z=z_{a}$ in the complex $z$-plane. We propose that these moduli are the positions in the $y$-direction of $r_{a}$ smooth monopoles that are located at $z=z_{a}$, along with conjugate angles. Again, the precise meaning of this statement holds when the $y$-positions in question are large. We will make this interpretation quantitative in Section 7, but for now we consider qualitative arguments.

A first motivation for this proposal comes from $\boldsymbol{m}$; we interpret the formula (2.27) as the sum of the magnetic charges of the singular monopoles at the boundary $\left(-\sum_{a} k_{a}\right)$ plus a contribution of 2 for every smooth monopole (contributing $2 \sum_{a} r_{a}$ in toto). The fact that the charge of the singular 
monopoles is negative is worthy of note. Prior to symmetry breaking, the charge of the singular monopoles does not have a meaningful sign - it is dual to a representation of $G^{\vee}=S U(2)$ — but after symmetry breaking, this sign makes sense and with our normalization it is negative.

To learn more, we recall some facts about the Bogomolny equations. For the Bogomolny equations on $\mathbb{R}^{3}$, the holomorphic data are the commuting operators $\mathcal{D}_{1}$ and $\mathcal{D}_{3}$, defined exactly as in equation (2.5). Localized monopole solutions of the Bogomolny equations manifest themselves in the holomorphic data through "bound states" in the parallel transport by $\mathcal{D}_{3}$, i.e., through normalizable solutions to

$$
\frac{\mathcal{D} s}{\mathcal{D} y}=0 .
$$

Such solutions only appear at specific positions in the $z$-plane, which are interpreted as the $z$ values of the monopole locations, because generically, if we pick $s$ to decay exponentially for $y \rightarrow-\infty$, it will grow exponentially for $y \rightarrow+\infty$.

In our setup, we are limited to $y \geq 0$, and an analogous normalizable solution exists precisely if the "small" section $s$ that vanishes for $y \rightarrow 0$ also decays exponentially at large $y$. If $\varphi$ also decays exponentially as well, then $s \wedge \varphi s$ vanishes for $y \rightarrow \infty$ and hence for all $y$. So sections $s$ that vanish at both ends can arise only only at zeroes of $K(z)$. Indeed, if

$$
s=\left(\begin{array}{l}
\widetilde{P} \\
\widetilde{Q}
\end{array}\right)
$$

near infinity, then given the form of (2.24), vanishing of $s$ for $y \rightarrow \infty$ is equivalent to $\widetilde{Q}=0$. Zeroes of $\widetilde{Q}$ are the same as zeroes of $Q$. (Indeed, $s \wedge \varphi s$ is independent of $y$; its zeroes are the zeroes of $Q$ if $y$ is small or of $\widetilde{Q}$ if $y$ is large.) So the relation to the ordinary Bogomolny equations does indeed suggest that there are $r_{a}$ smooth monopoles at each point $z=z_{a}$ at which $Q$ vanishes. The position of the smooth monopoles in the $y$-direction should be encoded in the values of $P$ and its first $r_{a}-1$ derivatives. We will make this picture more precise in Section 7; for now we simply observe that the normalizable small section $s\left(z_{a}\right)$ behaves as $P\left(z_{a}\right) \mathrm{e}^{-m_{1} y}$, which suggests that increasing $\left|P\left(z_{a}\right)\right|$ moves the smooth monopoles toward large $y$.

The limit $P\left(z_{a}\right) \rightarrow 0$ is somewhat singular, as $s$ is supposed to be everywhere nonzero. The physical picture suggests that the limit will correspond to a monopole bubbling situation, where the smooth monopole is pushed to the boundary, and screens the singular monopole's charge. Monopole 
bubbling is a phenomenon (originally described in [18] and rediscovered in Section 10.2 of [15]) in which an 't Hooft operator absorbs a smooth BPS monopole, lowering the magnitude of its magnetic charge.

\section{5 "Complex" symmetry breaking}

Now we consider the case of symmetry breaking in $\varphi$. Suppose, for $G=$ $S O(3)$, that the eigenvalues of $\varphi$ for $y \rightarrow \infty$ are $\pm \boldsymbol{a}$. Then, as $\varphi$ commutes with $\mathcal{D}_{y}$, its eigenvalues are $\pm \boldsymbol{a}$ everywhere. By a complex gauge transformation, we can reduce to the case that $\varphi$ is a constant diagonal matrix:

$$
\varphi=\left(\begin{array}{cc}
a & 0 \\
0 & -a
\end{array}\right)
$$

Writing as usual $s=\left(\begin{array}{l}P \\ Q\end{array}\right)$ for the small section, we find $s \wedge \varphi s=2 a P Q$. After putting $\varphi$ in the form (2.30), we can still make a gauge transformation by $\operatorname{diag}\left(\lambda, \lambda^{-1}\right)$, mapping

$$
P \rightarrow \lambda P, \quad Q \rightarrow \lambda^{-1} Q .
$$

In the presence of singular monopoles of charges $k_{a}$ located at $z=z_{a}$ (and $y=0)$, the condition we want to satisfy is $s \wedge \varphi s=\prod_{a=1}^{d}\left(z-z_{a}\right)^{k_{a}}:=K(z)$. This becomes

$$
2 a P Q=K(z)
$$

Solutions of these equations are associated to factorizations of $K(z)$ and (modulo a transformation (2.31)) have no moduli.

In the case of $d$ boundary 't Hooft operators that all have $k_{a}=1$, corresponding to minimum magnetic charge, the number of solutions is precisely $2^{d}$. The solutions correspond simply to the possible ways to distribute the factors of $K(z)$ between $P$ and $Q$. The number $2^{d}$ has a natural interpretation. On the Coulomb branch, we might expect a minimum charge 't Hooft operator to have two possible states, with its magnetic charge being aligned or anti-aligned with the symmetry breaking. The two states correspond to a zero in $P$ or a zero in $Q$. In the dual description by Chern-Simons theory with Wilson operators, a minimum charge 't Hooft operator corresponds to a Wilson operator in the two-dimensional representation of $S U(2)$; such an operator again represents two quantum states, with positive or negative electric charge along the axis of symmetry breaking. 
To confirm that the $2^{d}$ solutions correspond to two possible choices of the magnetic charge for each 't Hooft operator, let us compute the magnetic charges of these solutions. Suppose that $P$ is of degree $d_{1}$ and $Q$ of degree $d_{2}$, where $d_{1}+d_{2}=d$. The ratio $P / Q$ does not depend on the normalization of the small section $s$. This ratio has electric charge -1 in the low-energy abelian gauge theory; it is a section of the line bundle $\mathcal{M}^{-1}$. On the other hand, concretely, $P / Q$ has $d_{1}$ zeroes and $d_{2}$ poles on the $z$-plane. $(P / Q$ has neither a pole nor a zero at $z=\infty$, when understood as a section of $\mathcal{M}^{-1}$. In fact, by a complex gauge transformation, the solution can be put near $z=\infty$ in the form of the original Nahm pole solution (1.3), and in particular is independent of $z$. This trivializes $\mathcal{M}$ near $z=\infty$ and makes $P / Q$ independent of $z$.) So the line bundle $\mathcal{M}$ has degree $d_{2}-d_{1}$ and hence

$$
\boldsymbol{m}=d_{2}-d_{1}
$$

From this, we see that the 't Hooft operator at $z=z_{a}$ contributes either -1 or +1 to $\boldsymbol{m}$, depending on whether we place the factor of $z-z_{a}$ in $P$ or in $Q$.

To get some insight into why there are more solutions for $\boldsymbol{a} \neq 0$ than there are for $\boldsymbol{a}=0$, consider a slightly more general ansatz for $\varphi$ :

$$
\varphi=\left(\begin{array}{cc}
\boldsymbol{a} & p(z) \\
0 & -\boldsymbol{a}
\end{array}\right)
$$

For $\boldsymbol{a} \neq 0$, the polynomial $p(z)$ can be removed by an upper triangular gauge transformation, but now there is a smooth limit for $\boldsymbol{a}=0$. The condition $s \wedge \varphi s=K$ becomes

$$
2 a P Q+p Q^{2}=\prod_{a=1}^{d}\left(z-z_{a}\right) .
$$

For $\boldsymbol{a} \neq 0, p$ is irrelevant, as it can be eliminated by $P \rightarrow P-p Q / 2 \boldsymbol{a}$. For $\boldsymbol{a} \neq 0$, equation (2.35) has $2^{d}$ solutions (modulo a complex gauge transformation that preserves the form of $\varphi$ ), corresponding to factorizations of $K(z)$ as $(2 \boldsymbol{a} P+p Q) Q$. But all of these solutions have $P \sim 1 / \boldsymbol{a}$ except for the one solution with $Q=1$ (and $P=0$ ) that we found already in Section 2.3.

We interpret this as follows. In the presence of a minimum charge 't Hooft operator at a boundary point $z=z_{a}$, there is always a magnetic charge -1 localized near the boundary. The magnetic charge that is localized near the boundary is the same for all solutions because the form of the solution near the boundary is always given by a standard model solution (the one 
“ATMP-16-3-A5-GAI" — 2013/2/1 — 19:36 — page 964 — \#30

described in Section 3.6 of [14]), independent of everything else. In the case of a solution of (2.32) with $Q\left(z_{a}\right)=0$ and (therefore) $P\left(z_{a}\right) \neq 0$, there is in addition a smooth BPS monopole, with magnetic charge 2 , located at $z=z_{a}$ and at a value of $y$ that depends on $P\left(z_{a}\right)$. So the total magnetic charge associated to $z=z_{a}$ is $-1+2=1$. As $\boldsymbol{a} \rightarrow 0, P\left(z_{a}\right) \rightarrow \infty$ and the smooth monopole disappears to $y=\infty$. In this way, all of the $2^{d}$ solutions become equivalent for $\boldsymbol{a} \rightarrow 0$, even though they are different for $\boldsymbol{a} \neq 0$. As always, such a description in terms of smooth BPS monopoles is only precise in the limit that the monopoles are located at large values of $y$, so that the Bogomolny equations are a good approximation near their positions. As we explain most fully in Section 7 , this is the case exactly when $\boldsymbol{a}$ is very small (compared to the inverse distances $1 /\left(z_{a}-z_{b}\right)$ between the 't Hooft operators) so in particular the description by BPS monopoles becomes precise for $\boldsymbol{a} \rightarrow 0$.

In the opposite case that $\boldsymbol{a}$ is large compared to the inverse distances, the symmetry breaking is strong and the different singular monopoles on the boundary are so far separated that they do not significantly influence each other. In this case, the essential statement is simply that a single 't Hooft operator of minimal charge, in the presence of symmetry breaking with $\boldsymbol{a} \neq 0$, has two possible states, in which it looks like an 't Hooft operator of charge 1 or -1 in the effective low-energy theory. Given this, a system of $d$ widely separated 't Hooft operators of minimal charge naturally has $2^{d}$ possible states.

\subsubsection{Implications}

The counting of solutions for $\boldsymbol{a} \neq 0$ circumvents a difficulty that we encountered in Section 2.3. With $2^{d}$ classical solutions, the physical Hilbert space in the presence of $d$ 't Hooft operators of minimal charge will have dimension $2^{d}$. These $2^{d}$ states are potentially dual to the states of $d$ Wilson operators labeled by the two-dimensional representation of $S U(2)$. And the representations of the braid group on $d$ strands that are associated to the Jones polynomial can certainly be realized in a vector space of dimension $2^{d}$.

Given this, it is reasonable to expect that the stretching strategy sketched in figure 2 of Section 1.2 can work if $\boldsymbol{a}$ is generic, but that for $\boldsymbol{a}=0$, an attempt to simplify a classical solution by stretching in one direction leads to behavior along the lines suggested in figure 4 .

More generally, in the presence of an 't Hooft operator on the boundary of charge $k_{a}$, we would hope to find $k_{a}+1$ states of magnetic charge $-k_{a},-k_{a}+2,-k_{a}+4, \ldots, k_{a}$. Indeed, such an 't Hooft operator in $S O(3)$ gauge theory is dual to a Wilson operator in $S U(2)$ gauge theory associated 
"ATMP-16-3-A5-GAI" — 2013/2/1 — 19:36 — page 965 — \#31

KNOT INVARIANTS

to the representation of spin $k_{a} / 2$; this representation has dimension $k_{a}+1$, and its weights are as indicated. We get the right number of solutions with the right magnetic charges if in solving equation (2.32), we allow arbitrary factorizations of $K(z)$ with the zeroes split between $P$ and $Q$ in an arbitrary fashion. Unfortunately, we do not have a simple interpretation of the factorizations for which both $P$ and $Q$ vanish at $z=z_{a}$. After all, $s=\left(\begin{array}{l}P \\ Q\end{array}\right)$ is supposed to be everywhere nonzero. It seems possible that the factorizations in which $P$ and $Q$ have a common zero should be interpreted in terms of monopole bubbling, that is, as solutions of the equations in the presence of an 't Hooft operator of reduced charge $k_{a}-2, k_{a}-4$, etc. To develop this idea in detail is beyond the scope of the present paper.

Going back to the simpler case that all $k_{a}$ are equal to 1 , since the space of physical states has a promising dimension $2^{d}$, the next step could be to try to compute the action of the braid group and to extract the Jones polynomial. Before attempting that, we will describe a further deformation of the problem, which turns out to be illuminating. Among other things, with this deformation, we will get a nice behavior regardless of the charges of the 't Hooft operators.

\section{Analysis at general $t$}

\subsection{Some basics}

Still searching for a useful description for 't Hooft operators of arbitrary magnetic charges, we consider the possibility of deforming the equation that we are trying to solve to one that might behave more conveniently upon stretching along one direction.

A strong hint that there is a useful deformation comes by considering the relation of the extended Bogomolny equations (2.3), (2.4) to Hitchin's equations [20]. Hitchin's equations are equations on an oriented two-manifold $C$ for a connection $A$ and an adjoint-valued one-form $\phi$ :

$$
\begin{aligned}
F-\phi \wedge \phi & =0, \\
d_{A} \star \phi & =0, \\
d_{A} \phi & =0 .
\end{aligned}
$$

The moduli space of solutions of these equations, up to gauge transformation, is a hyper-Kahler manifold $\mathcal{M}_{H}(G, C)$. As a hyper-Kahler manifold, $\mathcal{M}_{H}(G, C)$ has a family of complex structures parameterized by a copy of 
$\mathbb{C P}^{1}$. The complex structures of $\mathcal{M}_{H}(G, C)$ have a simple interpretation. They correspond to ways of splitting the three real equations in (3.1) into two parts: two real equations, which can be combined to a single complex equation; and a third real equation that is "orthogonal" to the first two. The complex equation then describes the holomorphic data parameterizing $\mathcal{M}_{H}(G, C)$ in one of its complex structures, and the remaining real equation is a moment map condition.

An exceptional split corresponds to the case that the complex equation is made by combining together the last two equations in (3.1) to get the Higgs bundle equation

$$
\bar{\partial}_{A} \varphi=0
$$

A more generic split involves a complex parameter $\zeta$. For any $\zeta$, set

$$
\begin{aligned}
& \mathcal{D}_{z}^{\zeta}=\frac{D}{D z}-\zeta^{-1}\left[\phi_{z}, \cdot\right] \\
& \mathcal{D}_{\bar{z}}^{\zeta}=\frac{D}{D \bar{z}}+\zeta\left[\phi_{\bar{z}}, \cdot\right] .
\end{aligned}
$$

(We write here $\phi_{z}$ and $\phi_{\bar{z}}$ instead of $\varphi$ and $\bar{\varphi}$.) For any $\zeta$, the equation

$$
\left[\mathcal{D}_{z}^{\zeta}, \mathcal{D}_{\bar{z}}^{\zeta}\right]=0
$$

is equivalent to two real linear combinations of the Hitchin equations (3.1). The possible complex structures on $\mathcal{M}_{H}(G, C)$ are parameterized by $\zeta$, where we add a point at infinity to the $\zeta$ plane to make $\mathbb{C P}^{1}$. For every $\zeta$, one defines a complex structure $I_{\zeta}$ in which equation (3.4) is regarded as an equation governing holomorphic data; in this complex structure, the holomorphic variables are $\mathcal{A}_{z}^{\zeta}=A_{z}-\zeta^{-1} \phi_{z}$ and $\mathcal{A}_{\bar{z}}^{\zeta}=A_{\bar{z}}+\zeta \phi$, and equation (3.4) is holomorphic in those variables. The third linear combination of the equations is regarded in complex structure $I_{\zeta}$ as a moment map condition.

For generic $\zeta$, equation (3.3) simply says that the complex connection $\mathcal{A}^{\zeta}=\mathcal{A}_{z}^{\zeta} d z+\mathcal{A}_{\bar{z}}^{\zeta} d \bar{z}$ is flat. Once equation (3.3) is expressed in terms of $\mathcal{A}^{\zeta}$, it has no explicit dependence on $\zeta$. Thus, $\mathcal{M}_{H}(G, C)$ when regarded simply as a complex manifold in complex structure $I_{\zeta}$, without worrying about its Kahler metric, is independent of $\zeta$ for generic $\zeta$. The exceptional values of $\zeta$ are 0 and $\infty$. For example, for $\zeta \rightarrow 0$, we must multiply $\mathcal{D}_{z}^{\zeta}$ by $\zeta$, whence it reduces to $-\left[\phi_{z}, \cdot\right]$. Meanwhile, for $\zeta=0, \mathcal{D}_{\bar{z}}=D_{\bar{z}}$. So the $\zeta \rightarrow 0$ limit 
"ATMP-16-3-A5-GAI" — 2013/2/1 — 19:36 — page 967 — \#33

KNOT INVARIANTS

of equation (3.4) is the Higgs bundle equation (3.2). By similar reasoning, the $\zeta \rightarrow \infty$ limit of (3.4) is the complex conjugate of (3.2).

The Higgs bundle equation has played a prominent role in our analysis because in Section 2.1, we derived the holomorphic data at $y \neq 0$ from the equation $\left[\mathcal{D}_{1}, \mathcal{D}_{2}\right]=0$, which was none other than the Higgs bundle equation. (The moment map condition in that analysis condition was not the usual moment map equation for Higgs bundles - namely the first equation in (3.1) - but rather it was the three-dimensional equation (2.4).) At this point, we are led to wonder whether we could modify the construction that led to $(2.3)$ so that the holomorphic data at $y \neq 0$ will be given not by a Higgs bundle but by the solution of a different complex linear combination of Hitchin's equations. It is in fact possible to do so. Indeed the path to doing so is not quite uniquely determined.

There is one particularly nice parameter by which we can vary the underlying four-dimensional equations. This is simply the parameter $t$ in equation (1.1). In addition to changing the equations, we should consider the possibility of changing the boundary conditions. Of the six fields $(\vec{A}, \vec{\phi})$ (that is, the components of $A$ and $\phi$ that are tangent to the boundary), only $\vec{\phi}$ has a singularity at $y=0$ in the basic Nahm pole solution (1.3). However, once one drops the dependence on the spatial coordinates $\vec{x}, \mathcal{N}=4$ super Yang-Mills theory has an $S O(6)$ symmetry that rotates the six components of $\vec{A}$ and $\vec{\phi}$. Therefore, one can obey the classical Yang-Mills equations with a solution obtained by applying an $S O(6)$ rotation to the Nahm pole (such a rotated Nahm pole was studied in [19], Section 4). Of course, the rotation in general will change the unbroken supersymmetry. It turns out, however (see Appendices A and B for more detail), that as long as the rotation matrix is contained in a certain $S U(3)$ subgroup of $S O(6)$, the unbroken supersymmetry is unchanged, and if it is contained in a certain $U(3)$ subgroup, then the unbroken supersymmetry changes in a way that corresponds to a change in the parameter $t$ in the four-dimensional equation (1.1). So there is considerable freedom in rotating the boundary condition, with or without a change in $t$.

After making a choice along the lines just described, the deformed equations and boundary conditions have a three-dimensional reduction that takes the familiar form of (2.1) and (2.2), but with a different definition of the $\mathcal{D}_{i}$. Deferring most of the details to the appendices, we will summarize some formulae that arise in an illuminating special case. If one wishes to preserve the $S O(3)$ symmetry of rotations of the boundary, then the rotation of the Nahm pole can only depend on a single parameter: the polar part of $\vec{A}$ must be a constant $\zeta$ times the polar part of $\vec{\phi}$. It turns out that such a boundary 
condition is compatible with the four-dimensional equations (1.1), with a modified value of $t$ that depends on $\zeta$.

In the reduction to three dimensions, we must now impose $A_{1}-\zeta \phi_{1}=$ $0=\phi_{y}$. The three commuting differential operators $\mathcal{D}_{i}$ then take the form

$$
\begin{aligned}
& \mathcal{D}_{1}=2 \frac{D}{D \bar{z}}+2 \zeta[\bar{\varphi}, \cdot], \\
& \mathcal{D}_{2}=-2 \zeta \frac{D}{D z}+2[\varphi, \cdot], \\
& \mathcal{D}_{3}=\frac{\mathcal{D}}{\mathcal{D} y}
\end{aligned}
$$

Here, as described in the appendix, $\mathcal{A}_{y}$ is an appropriate linear combination of $A_{y}$ and $\phi_{1}$. The condition that the $\mathcal{D}_{i}$ commute must be supplemented by a moment map condition, which is also described in the appendix. As one would anticipate for a geometry that preserves four supercharges, the commutativity constraint $\left[\mathcal{D}_{i}, \mathcal{D}_{j}\right]=0$ can be derived from a superpotential, which in fact is a multiple of the Chern-Simons function:

$$
\mathcal{W}=\frac{1}{4 \pi \mathrm{i}} \int_{W} \operatorname{Tr}\left(\mathcal{A} \wedge d \mathcal{A}+\frac{2}{3} \mathcal{A} \wedge \mathcal{A} \wedge \mathcal{A}\right)
$$

To be more exact, in varying $\mathcal{W}$ to derive the equations $\left[\mathcal{D}_{i}, \mathcal{D}_{j}\right]=0$, one imposes a constraint on the variation of $\mathcal{A}$ at $y=0$, so as to avoid delta function terms in the variation of $\mathcal{W}$.

The holomorphic data away from $y=0$ are now easy to describe. We recover the picture studied in Section 2 if $\zeta=0$, but as soon as $\zeta$ is nonzero (and not infinite), the three commuting operators $\mathcal{D}_{i}$ simply describe a complex flat connection on $C \times \mathbb{R}_{+}$. The covariant derivatives for this flat connection are $\mathcal{D}_{z}=\mathcal{D}_{1} / 2, \mathcal{D}_{\bar{z}}=-\mathcal{D}_{2} / 2 \zeta, \mathcal{D}_{y}=\mathcal{D}_{3}$. So commuting operators $\mathcal{D}_{i}$ simply describe a complex flat connection. We will call the flat connection $\mathcal{A}$, irrespective of how it was defined in terms of $A$ and $\phi$. $\mathcal{A}$ is constrained by a moment map condition, which does not quite coincide with the most commonly studied moment map condition for a complex flat connection [21], although it is qualitatively similar; we expect it to have a unique solution for any $\zeta$.

The holomorphic data away from $y=0$ are hence simply a complex flat connection on $C \times \mathbb{R}_{+}$, or equivalently, since this space is contractible to $C$, a complex flat connection on $C$. We will mainly be interested in the case that $C$ is $\mathbb{R}^{2}$ or $\mathbb{C P}^{1}$. In either case, $C$ is simply-connected, so a 
complex flat connection on $C$ is trivial. One may wonder therefore how anything of interest can happen. The answer is that most of the structure of interest will come from the boundary condition at $y=0$. In the case of symmetry breaking, there is also some interesting structure in the behavior at $y=\infty$.

\subsection{Nahm poles and opers}

The first point is to understand how a complex flat connection can have a Nahm pole. The answer is that the pole appears in $\mathcal{A}_{y}$ and $\mathcal{A}_{z}$, but not in $\mathcal{A}_{\bar{z}}$. The model example of a flat connection with a Nahm pole is

$$
\begin{aligned}
& \mathcal{A}_{z}=\frac{\boldsymbol{t}_{+}}{y}, \\
& \mathcal{A}_{\bar{z}}=0 \\
& \mathcal{A}_{y}=\frac{\boldsymbol{t}_{3}}{y} .
\end{aligned}
$$

This describes a flat connection as long as $\left[\boldsymbol{t}_{3}, \boldsymbol{t}_{+}\right]=\boldsymbol{t}_{+}$. We are interested in the case that $\boldsymbol{t}_{+}=\boldsymbol{t}_{1}+\mathrm{i} \boldsymbol{t}_{2}$, where the $\boldsymbol{t}_{i}, i=1,2,3$ generate a principal $\mathfrak{s u}(2)$ subalgebra of $\mathfrak{g}$.

Being flat, this connection can be described by a formula $d+\mathcal{A}=g d g^{-1}$. For example, for $\mathfrak{s u}(2)$, we can take explicitly

$$
g=\left(\begin{array}{cc}
y^{-1 / 2} & -z y^{-1 / 2} \\
0 & y^{1 / 2}
\end{array}\right),
$$

which leads to

$$
\begin{aligned}
& \mathcal{A}_{z}=\left(\begin{array}{ll}
0 & 1 \\
0 & 0
\end{array}\right) \frac{1}{y}, \\
& \mathcal{A}_{\bar{z}}=0, \\
& \mathcal{A}_{y}=\left(\begin{array}{cc}
1 & 0 \\
0 & -1
\end{array}\right) \frac{1}{2 y} .
\end{aligned}
$$

Alternatively, the model solution can be generated from the nonsingular flat connection

$$
\mathcal{A}_{z}=\left(\begin{array}{ll}
0 & 1 \\
0 & 0
\end{array}\right), \quad \mathcal{A}_{\bar{z}}=\mathcal{A}_{y}=0
$$


“ATMP-16-3-A5-GAI" — 2013/2/1 — 19:36 — page $970-\# 36$

by the same singular gauge transformation as in (2.10):

$$
g=\left(\begin{array}{cc}
y^{-1 / 2} & 0 \\
0 & y^{1 / 2}
\end{array}\right) .
$$

We now proceed rather as we did in the Higgs bundle case to explain the condition that must be placed on a complex flat bundle $E$ so that it can be placed in the form (3.9) near $y=0$, modulo less singular terms. We write simply $E$ for the restriction of $E$ to $C=C \times\{y\}$ for some fixed $y>0$. We consider solutions $s$ of the equation $\mathcal{D}_{y} s=0$ that vanish as $y^{1 / 2}$ for $s \rightarrow 0$. Sections $s$ obeying these conditions span a rank one sub-bundle $L \subset E$. In the case of the model solution, any such $s$ is a multiple of

$$
s=y^{1 / 2}\left(\begin{array}{l}
0 \\
1
\end{array}\right)
$$

so as in the Higgs bundle case, $L$ is simply spanned by sections whose upper component vanishes.

Also as before, if we regard $E$ as a flat bundle over $C$, then $L$ is a holomorphic sub-bundle; indeed, the object $s$ that we have just defined is a holomorphic section of $L$, since it is certainly annihilated by $\mathcal{D}_{\bar{z}}$. However, it is not true that $s$ is annihilated by $\mathcal{D}_{z}$. On the contrary, a look at the previous formulae shows at once that

$$
s \wedge \mathcal{D}_{z} s=1 .
$$

This brings us to the mathematical notion of an "oper". (For an explanation of this notion as well as a review of many related ideas that will enter our story later, see $[22,23]$.) For $G=S U(2)$, an oper is a flat rank two complex bundle $E$ bundle over a Riemann surface $C$, with structure group $S L(2, \mathbb{C})$, together with a holomorphic line sub-bundle $L \subset E$ with the following property: $L$ is nowhere invariant under parallel transport by $\mathcal{D}_{z}$. The last statement means the following. If $s$ is a local nonzero holomorphic section of $L$, then $\mathcal{D}_{z} s$, which will be $E$-valued since $\mathcal{D}_{z}$ is a connection on $E$, is nowhere $L$-valued. An equivalent statement, since $L$ is spanned by multiples of $s$, is that $\mathcal{D}_{z} s$ is nowhere a multiple of $s$. Alternatively, $s \wedge \mathcal{D}_{z} s$ is everywhere nonzero. The last statement does not depend on the choice of the nonzero section $s$, since if we replace $s$ by $f s$ (where $f$ is a nonzero local holomorphic function on $C$ ), we have $s \wedge \mathcal{D}_{z} s \rightarrow$ $f^{2} s \wedge \mathcal{D}_{z} s$ 
We have extracted an oper structure from the Nahm pole boundary conditions; conversely let us see that given an oper on $C$, that is a pair $(E, L)$ obeying the conditions just described, we can construct a solution of the Nahm pole boundary conditions. Go to a gauge in which $L$ is spanned by vectors whose upper component vanishes. Holomorphy of $L$ means that $\mathcal{A}_{\bar{z}}$ is lower triangular in this gauge. The oper condition $s \wedge \mathcal{D}_{z} s \neq 0$ for any nonzero local section of $s$ implies that in this gauge, the upper right matrix element of $\mathcal{A}_{z}$ is nonzero. By a further diagonal gauge transformation, we can set this matrix element to 1 . Then we pull back the flat bundle $E$ with connection $\mathcal{A}$ from $C$ to $C \times \mathbb{R}_{+}$(to get a flat connection on $C \times \mathbb{R}_{+}$with no dependence on $y$ ) and make the singular gauge transformation (3.11). Having an upper right matrix element of 1 means that after the singular gauge transformation, $\mathcal{A}_{z}$ has the singular behavior of the model solution (3.9); being lower triangular, $\mathcal{A}_{\bar{z}}$ acquires no singularity. Finally, the gauge transformation gives $\mathcal{A}_{y}$ precisely the form of the model solution.

So we have shown, at least for the case that $G$ has rank 1, that twodimensional opers correspond precisely to solutions of the Nahm pole boundary conditions in three dimensions modulo less singular terms. Conjecturally, by a further smooth complex-valued gauge transformation, one can satisfy the moment map condition.

\subsubsection{Some further remarks}

We add the following technical remarks. Since we want to be able to consider 't Hooft operators of minimum charge, we will take the gauge group in the rank 1 case to be $G=S O(3)$, rather than $S U(2)$. Accordingly, we should restate the above derivation in terms of the adjoint bundle ad $(E)$ rather than $E$. Because our considerations have been local on $C$, rewriting the construction in terms of the adjoint bundle does not change very much and we will omit it. (The main difference is that what can be naturally defined globally is in general not $L$ but $L^{2}$, which is a sub-bundle of $\operatorname{ad}(E)$.)

Also, everything we have said for $G$ of rank 1 has an analog for any semisimple $G$, somewhat as we indicated in the Higgs bundle case at the end of Section 2.2. For example, for $G=S U(n)$, an oper is a flat complex bundle $E \rightarrow C$ of rank $n$ with $S L(n, \mathbb{C})$-valued holonomies together with a line subbundle $L \subset E$ that is holomorphic and has the property that if $s$ is a local nonzero holomorphic section of $L$, then $s, \mathcal{D}_{z} s, \ldots, \mathcal{D}_{z}^{n-1} s$ furnish a local trivialization of $E$. The equations $\left[\mathcal{D}_{i}, \mathcal{D}_{j}\right]=0$ together with the Nahm pole boundary condition determine such an oper structure, by arguments similar to those we have already given. 
"ATMP-16-3-A5-GAI" — 2013/2/1 — 19:36 — page 972 — \#38

\subsection{Opers with singularities}

Now we would like to modify the Nahm pole boundary condition to incorporate additional singularities - which we will associate with 't Hooft operators - on the boundary at $y=0$.

The type of singularity that we want can be guessed by analogy with the discussion of Higgs bundles. We will still have a flat $G_{\mathbb{C}}$ bundle $E \rightarrow C \times \mathbb{R}_{+}$, and near a generic boundary point, the flat connection will look like the model solution (3.7), up to a unitary (that is, $G$-valued rather than $G_{\mathbb{C}^{-}}$ valued) gauge transformation. We can still define a holomorphic line subbundle $L \subset E$ by considering sections $s$ obeying $\mathcal{D}_{y} s=0$ and vanishing for $y \rightarrow 0$; and we still require that if $s$ is a local holomorphic section of $s$, then $s \wedge \mathcal{D}_{z} s$ is generically nonzero.

The only difference is that now we assume the existence of exceptional points $z_{a}, a=1, \ldots, d$, at which $s \wedge \mathcal{D}_{z} s$ vanishes. In fact, we specify positive integers $k_{a}$ and require that $s \wedge \mathcal{D}_{z} s$ vanishes in order $k_{a}$ for $z \rightarrow z_{a}$ :

$$
s \wedge \mathcal{D}_{z} s \sim\left(z-z_{a}\right)^{k_{a}} .
$$

This is analogous to requiring $s \wedge \varphi s \sim\left(z-z_{a}\right)^{k_{a}}$ in the Higgs bundle case.

To get a precise problem of classical or quantum gauge theory with this sort of boundary behavior, what remains is to specify precisely what sort of singularity a solution of the moment map condition $\sum_{i}\left[\mathcal{D}_{i}, \mathcal{D}_{i}^{\dagger}\right]=0$ (or the four- or five-dimensional equations that can be dimensionally reduced to it) is supposed to have at $z=z_{a}$. As in most such problems, to do this one finds a model solution for the case of only one singularity, at, say, $z=0$ (and $y=0$ ) and with an arbitrary $k$. Then one asks that the singular behavior of the solution near each of the points $z=z_{a}, y=0$ should coincide with that of the model solution, for $k=k_{a}$. For the case of Higgs bundles, the appropriate model solutions were found (for $G$ of rank 1 ) in Section 3.6 of [14], but for the generalization considered here, at present we are only able to find the model solutions numerically. They are described in Appendix C.

The objects that we have described so far correspond to solutions of the flatness and moment map conditions on $C \times \mathbb{R}_{+}$with boundary conditions associated to Nahm poles or opers, except at finitely many boundary points where the oper condition is corrected. In particular, as soon as one gets away from $y=0$, one simply has a flat bundle (with a moment map condition). The monodromy of the flat bundle around the points $z=z_{a}$ is therefore trivial, so the exceptional behavior at the points $z=z_{a}$ only affects the oper 
property of the pair $(E, L)$, not the flatness of $E$. Singularities of this kind are called oper singularities with trivial monodromy.

\subsection{Oper singularities and Bethe equations}

Let us now make concrete (referring to [22] for much more detail) what sort of an object is an oper with monodromy-free singularities. We will make this analysis for the case that $C$ is simply-connected, so that there are no moduli in the choice of the flat bundle $E \rightarrow C$. So $C$ will be either $\mathbb{R}^{2}$ or $\mathbb{C P}^{1}$; that is, it will be the complex $z$-plane with or without an added point at infinity. There are two reasons for assuming $C$ to be simply connected: this is the most relevant case for understanding the Jones polynomial; and also, eliminating the choice of $E$ from the discussion will make it easier to focus on the opers and their singularities.

Since $C$ is simply connected, a flat bundle over $C$ is trivial. So we can go to a gauge with $\mathcal{A}_{z}=\mathcal{A}_{\bar{z}}=0$. The line sub-bundle $L$ of $E$ is inevitably trivial for $C=\mathbb{R}^{2}$, or trivial after omitting the point $z=\infty$ for $C=\mathbb{C P}^{1}$. So it is globally generated by a section $s$, but we cannot put $s$ in a simple form while also setting $\mathcal{A}_{z}=\mathcal{A}_{\bar{z}}=0$. Instead, we take

$$
s=\left(\begin{array}{l}
P(z) \\
Q(z)
\end{array}\right)
$$

with polynomials $P$ and $Q$. $P$ and $Q$ are uniquely determined up to a linear transformation

$$
\left(\begin{array}{l}
P \\
Q
\end{array}\right) \rightarrow M\left(\begin{array}{l}
P \\
Q
\end{array}\right), \quad M \in G L(2, \mathbb{C})
$$

Now $s \wedge \mathcal{D}_{z} s$ reduces to $P \partial_{z} Q-Q \partial_{z} P$. So if the polynomial $K(z)=$ $\prod_{a=1}^{d}\left(z=z_{a}\right)^{k_{a}}$ encodes the positions and charges of the oper singularities, then the equation we would like to solve is

$$
P \partial_{z} Q-Q \partial_{z} P=K(z)
$$

modulo the action of $S L(2, \mathbb{C})$. (Choosing $K$ to have leading coefficient 1 has reduced $G L(2, \mathbb{C})$ to $S L(2, \mathbb{C})$.) It is convenient to fix the action of two of the three generators of $S L(2, \mathbb{C})$ by requiring that the degree of the polynomial $Q$ is less ${ }^{6}$ than the degree of $P$ (if two polynomials have the

\footnotetext{
${ }^{6}$ Later on, in the presence of symmetry breaking, we will have to relax this condition.
} 
same degree, a linear combination of them has smaller degree and we call this $Q$ ), and that $Q$ has leading coefficient 1,

$$
Q(z)=\prod_{i=1}^{q}\left(z-w_{i}\right)
$$

for some $w_{i}$. These conditions leave only the freedom to add to $P$ a multiple of $Q$.

We can recast (3.17) as

$$
\partial_{z} \frac{P}{Q}=-\frac{K(z)}{Q^{2}}
$$

The left-hand side of this equation has zero residues at the zeroes $w_{i}$ of $Q(z)$. The right-hand side must also have zero residues. This gives the constraints

$$
\sum_{a} \frac{k_{a}}{w_{i}-z_{a}}=\sum_{j \neq i} \frac{2}{w_{i}-w_{j}}, \quad i=1, \ldots, q
$$

As usual, we take $K(z)=\prod_{a}\left(z-z_{a}\right)^{k_{a}}$. Vice versa, given a solution of these equations, the residues of $K / Q^{2}$ are zero; hence $\int K / Q^{2} d z$ is a rational function $P / Q$, and $P$ is fixed up to a constant multiple of $Q$, which is the expected indeterminacy.

If $C=\mathbb{R}^{2}$, opers with the desired monodromy-free singularities simply correspond to the solutions of equations (3.20). For $C=\mathbb{C P}^{1}$, we must further ensure that the oper does not have an additional singularity at infinity. The condition for this turns out to be that the degree $q$ of the polynomial $Q$ is just one-half of the degree of $K$ :

$$
q=\frac{k}{2}, \quad k=\sum_{a} k_{a}
$$

To determine whether the oper has a singularity at infinity, let $p$ be the degree of $P$ and define $\left(\begin{array}{c}\widetilde{P} \\ \widetilde{Q}\end{array}\right)=z^{-p}\left(\begin{array}{l}P \\ Q\end{array}\right)$. We view $\widetilde{P}, \widetilde{Q}$ as polynomials in $v=1 / z$. The condition that the degree of $P$ exceeds the degree $q$ of $Q$ implies that there is no cancellation of the leading power of $z$ on the left-hand 
"ATMP-16-3-A5-GAI" — 2013/2/1 — 19:36 — page 975 — \#41

KNOT INVARIANTS

side of (3.19) and hence that $p+q=k+1$. Since $q<p$, it follows that

$$
q \leq \frac{k}{2}
$$

in general. The condition that the oper has no singularity at $z=\infty$ or $v=0$ is that $\left(\widetilde{P} \partial_{v} \widetilde{Q}-\widetilde{Q} \partial_{v} \widetilde{P}\right)_{v=0} \neq 0$, and it is not hard to see that this coincides with (3.21).

To get farther, we need the theory of integrable systems. Rather "miraculously", equations (3.20) are the Bethe equations of an integrable model, which is the Gaudin model or a certain large impurity limit of the XXX spin chain. (The connection between opers with monodromy-free singularities and the Gaudin model is reviewed in [22], following earlier developments such as $[24,25]$. For more on this, see Section 8.6.) For $a=1, \ldots, d$, let $R_{a}$ be a copy of the representation of $S U(2)$ of spin $j_{a}=k_{a} / 2$, and let $\mathcal{H}=\otimes_{a=1}^{d} R_{a}$. The Hamiltonians of the Gaudin model are the commuting operators on $\mathcal{H}$ given by

$$
H_{a}=\sum_{b \neq a} \frac{\vec{T}_{a} \cdot \vec{T}_{b}}{z_{a}-z_{b}}
$$

Here $\vec{T}_{a}$ are the generators of $\mathfrak{s u}(2)$ acting on $R_{a}$, and $\vec{T}_{a} \cdot \vec{T}_{b}$ is the inner product of $\vec{T}_{a}$ and $\vec{T}_{b}$ (defined with the quadratic form such that $\vec{T}_{a} \cdot \vec{T}_{a}=$ $\left.j_{a}\left(j_{a}+1\right)\right)$. Actually, what we have written in (3.23) are the Hamiltonians for the Gaudin model for $G^{\vee}=S U(2)$. (We call this group $G^{\vee}$ as it is naturally dual to the gauge group $G$ that appears in the rest of our analysis.) There is a Gaudin model for any $G^{\vee}$, and it bears the same relation to opers that we are about to describe for $S U(2)$, but if $G^{\vee}$ has rank bigger than 1, then the $H_{a}$ are only part of a complete set of commuting Hamiltonians.

Since the Gaudin Hamiltonians commute, they can be simultaneously diagonalized. Moreover, since they commute with the action of $G^{\vee}$, their joint eigenvectors can be organized in irreducible representations of $G^{\vee}$. Because of the $G^{\vee}$ action, to understand all of the joint eigenvectors of the Gaudin Hamiltonians, it suffices to understand those joint eigenvectors that are also highest weight vectors for the action of $G^{\vee}$.

In the theory of the Bethe ansatz for the Gaudin model of $G^{\vee}=S U(2)$, it is shown that solutions of the Bethe equations (3.20) correspond to the joint eigenvectors that are also highest weight vectors for the action of $G^{\vee}$. 
In this correspondence, the weight $\boldsymbol{w}$ is related to the degree $q$ of $Q$ by

$$
\boldsymbol{w}=\frac{k}{2}-q
$$

In particular, if we want $G^{\vee}$-invariant joint eigenvectors of the Gaudin Hamiltonians, we need $\boldsymbol{w}=0$ and $q=k / 2$; but as we observed in (3.21), this is the condition that the corresponding oper extends over $\mathbb{C P}^{1}$ with no singularity at infinity.

So the number of opers on $\mathbb{C P}^{1}$ with monodromy-free singularities is the same as the number of $S U(2)$-invariant joint eigenvectors of the Gaudin Hamiltonians. But the joint eigenvectors of the commuting Gaudin Hamiltonians are a basis for $\mathcal{H}$, and similarly the $G^{\vee}$-invariant joint eigenvectors are a basis for $\mathcal{H}^{G^{\vee}}$, the $G^{\vee}$-invariant part of $\mathcal{H}$. So the number of opers that obey the conditions that we have imposed is precisely the dimension of $\mathcal{H}^{G^{\vee}}$.

This result is our first concrete success in comparing the counting of BPS solutions in $G$ gauge theory to Chern-Simons theory with gauge group $G^{\vee}$. Consider Chern-Simons theory on $\mathbb{C P}^{1}$ with charges in the representations $R_{a}, a=1, \ldots, d$. We place these charges at points $z_{a} \in \mathbb{C P}^{1}$. In the classical limit, the space of physical states is just the $G^{\vee}$-invariant part of $\mathcal{H}=\otimes_{a} R_{a}$; the restriction to $G^{\vee}$-invariant states is the Gauss law constraint. This also gives the right answer for the dimension of the physical Hilbert space of Chern-Simons theory if the Chern-Simons coupling parameter $\boldsymbol{k}^{\vee}$ is generic. On the other hand, in the dual description in which the Hilbert space is constructed starting with time-independent solutions in $G$ gauge theory, the states should correspond, ${ }^{7}$ from the arguments we have given, to opers on $\mathbb{C P}^{1}$ with singularities of charge $k_{a}$ at the $z_{a}$. Since the number of these opers is the same as the dimension of $\mathcal{H}^{G^{\vee}}$, we have at least succeeded in reconciling the dimensions of the spaces of physical states in the two descriptions. This gives an indication that with the help of the deformation that we have exploited in the present section to $\zeta \neq 0$, the program of Section 1.2 based on stretching a knot in one direction can actually work.

The counting of states is less transparent if we take $C=\mathbb{R}^{2}$ rather than $\mathbb{C P}^{1}$. Qualitatively, it is clear that the number of physical states in ChernSimons theory is larger on $\mathbb{R}^{2}$ than on $\mathbb{C P}^{1}$, because, as the flux can escape to infinity, a physical state need not be completely gauge-invariant. However, to understand the condition that should be imposed at infinity is

\footnotetext{
${ }^{7}$ We explain in Section 4.1 why time-dependent instanton corrections in the $G$ gauge theory do not affect this counting of states.
} 
rather delicate, and it is hard to understand in $G^{\vee}$ Chern-Simons theory the result that seems to come from the opers: physical states on $\mathbb{R}^{2}$ correspond to highest weight vectors in $\mathcal{H}$. After incorporating symmetry breaking in Section 3.5, the comparison between the two descriptions will be simpler.

\subsubsection{Relation to conformal field theory}

In arriving at the Gaudin model, we have accomplished much more than simply getting a number of classical solutions that is reminiscent of known constructions of the Jones polynomial. The Jones representations of the braid group can be described [26] as the monodromy of the KnizhnikZamolodchikov equations [27]. These equations are as follows. Express the usual parameter $q$ that enters the Jones polynomial as $q=\exp \left(2 \pi i /\left(\boldsymbol{k}^{\vee}+\right.\right.$ $2)$ ). Let $\mathcal{B}$ be the space of distinct $d$-plets $z_{1}, \ldots, z_{d} \in \mathbb{C}$. And let $\mathcal{H}^{*}$ be the trivial bundle over $\mathcal{B}$ with fiber $\mathcal{H}=\otimes_{a=1}^{d} R_{a}$. The Knizhnik-Zamolodchikov equations are the following system of equations for a section $\Theta$ of $\mathcal{H}^{*}$ :

$$
\left(\frac{\partial}{\partial z_{a}}+\frac{H_{a}}{\boldsymbol{k}^{\vee}+2}\right) \Theta=0 .
$$

The $H_{a}$ are the Gaudin Hamiltonians (3.23). The Knizhnik-Zamolodchikov equations describe parallel transport of the section $\Theta$ of $\mathcal{H}^{*}$ with respect to a certain flat connection, which is implicitly defined in (3.25). To verify flatness of the connection, one uses the fact that the $H_{a}$ commute and also the relation $\partial H_{b} / \partial z_{a}=\partial H_{a} / \partial z_{b}$. The solutions $\Theta$ of the KnizhnikZamolodchikov equation are conformal blocks of two-dimensional current algebra with symmetry group $G^{\vee}$; they are important in two-dimensional conformal field theory.

Since opers with monodromy-free singularities correspond to a basis for $\mathcal{H}$, we will, in our approach to the Jones polynomial, eventually be using gauge theory to construct a flat connection on the bundle $\mathcal{H}^{*}$; moreover, as we hope to recover the Jones representations of the braid group, this flat connection should be gauge-equivalent to the one defined by the KnizhnikZamolodchikov equations. Actually, this tempting-sounding route is not the one we will follow. The very same Jones representations of the braid group have another realization in conformal field theory in terms of Virasoro conformal blocks for correlators of a product of degenerate fields and this will prove more useful. 
“ATMP-16-3-A5-GAI" — 2013/2/1 — 19:36 — page 978 — \#44

\subsection{Symmetry breaking again}

Just as in Section 2.4, we can gain some further clarity by moving away from the origin of the Coulomb branch. As always, we do so by turning on constant and commuting expectation values for $\vec{\phi}$ near $y=\infty$. In the present context, this means that the connection form $\mathcal{A}$ does not vanish at infinity, but is a one-form with constant coefficients; moreover, these coefficients commute with each other.

\subsection{1 "Real" symmetry breaking}

First we consider the case that only $\mathcal{A}_{y}$ has an expectation value at infinity. This expectation value arises from the value of $\phi_{1}$ at infinity, and so just as in (2.24) we have

$$
\mathcal{A}_{y}=\left(\begin{array}{cc}
a_{1} & 0 \\
0 & -a_{1}
\end{array}\right), \quad y \rightarrow \infty
$$

where we can take $\boldsymbol{a}_{1}>0$.

The condition that $\mathcal{A}_{z}$ and $\mathcal{A}_{\bar{z}}$ should have no exponential growth at infinity tells us that they must be upper triangular in a gauge in which $\mathcal{A}_{y}$ looks like (3.26) for $y \rightarrow \infty$. Thus, near $y=\infty$, the real symmetry breaking gives us a natural way to put the whole flat connection in a triangular form.

An oper that is endowed with a covariantly constant reduction of its structure group to the group of upper triangular matrices - that is, to a Borel subgroup - is called a Miura oper. This notion is described in detail in [22]. Any oper bundle without monodromy can be given a Miura oper structure; in fact, there is a one-parameter family of ways to do so. Concretely, if the rank two flat bundle $E \rightarrow C$ has trivial monodromy, then the associated bundle of $\mathbb{C P}^{1}$ 's (whose fibers are obtained by projectivizing the fibers of $E)$ also carries a flat connection without monodromy. Let us call this bundle $\mathcal{B}$. Picking an arbitrary section of $\mathcal{B}$ over some given point $p \in C$ and parallel transporting it, we get a covariantly constant section of $\mathcal{B}$ which turns the underlying oper into a Miura oper. This procedure introduces one complex modulus - the choice of a point in the fiber of $\mathcal{B}$ over the starting point $p$. This means that a Miura oper without monodromy depends on a complex modulus. (When — as in Section 3.5.2 - we introduce symmetry breaking in a complex direction, this modulus will disappear, because there will be no freedom to make a gauge rotation of $\mathcal{A}_{y}$ at infinity relative to $\mathcal{A}_{z}$.) 
"ATMP-16-3-A5-GAI" — 2013/2/1 — 19:36 — page 979 — \#45

KNOT INVARIANTS

The Bethe roots have a particularly nice interpretation in the case of a Miura oper. To explain this most simply, let us go back to the case that $C=\mathbb{R}^{2}$ and use a gauge with $\mathcal{A}_{z}=\mathcal{A}_{\bar{z}}=0$. The behavior for $y \rightarrow \infty$ singles out a sub-bundle $\widetilde{L}$ of the rank 2 bundle $E$ that is invariant under parallel transport; we may call it a flat sub-bundle. In a gauge with the asymptotic behavior (3.26), $\widetilde{L}$ is generated by a covariantly constant section $\widetilde{s}$ that vanishes for $y \rightarrow \infty$. After a complex gauge transformation to set $\mathcal{A}_{z}=\mathcal{A}_{\bar{z}}=0, \widetilde{s}$ is simply constant; we may as well take

$$
\widetilde{s}=\left(\begin{array}{l}
1 \\
0
\end{array}\right)
$$

On the other hand, the behavior for $y \rightarrow 0$ determines a holomorphic (not flat) sub-bundle $L \subset E$, generated as before (in a gauge $\mathcal{A}_{z}=\mathcal{A}_{\bar{z}}=0$ ) by

$$
s=\left(\begin{array}{l}
P \\
Q
\end{array}\right)
$$

The choice of the Miura structure $\widetilde{s}$ gives a way to pick a natural linear combination of the components of $s$, namely

$$
\widetilde{s} \wedge s=Q
$$

The $Q$ determined this way is not necessarily the one that we used in Section 3.4, where we took $Q$ to be a linear combination of components of $s$ with minimum degree; now $Q$ is simply determined by the Miura structure. With our new choice, the zeroes of $Q$ have a simple interpretation: they are the points at which $s$ is a multiple of $\widetilde{s}$. In other words, the sub-bundle determined by the behavior for $y \rightarrow \infty$ is generically different from the subbundle determined by the behavior for $y \rightarrow 0$. The zeroes of $Q-$ which are called Bethe roots - are precisely the points at which these coincide. Another way to say the same thing is that the Bethe roots are the values of $z$ at which there is a solution of $\mathcal{D}_{y} s=0$ that vanishes for both $y \rightarrow 0$ and $y \rightarrow \infty$. As we discussed in Section 2.4, in the context of the Bogomolny equations one would say that there are smooth BPS monopoles at those values of $z$ (and some values of $y$ ).

For a concrete example, suppose that there are no 't Hooft operators at all. The flat bundle $E \rightarrow \mathbb{R}^{2}$ is completely trivial and it has up to isomorphism 
a unique oper structure with

$$
s=\left(\begin{array}{l}
z \\
1
\end{array}\right)
$$

In the absence of symmetry breaking, our convention that $Q$ is the linear combination of components of $s$ with smaller degree leads to $Q=1$, and hence (up to the freedom of adding to $P$ a multiple of $Q$ and rescaling it) $P=z$.

In the presence of real symmetry breaking, the Miura structure gives a distinguished choice (3.29) of $Q$ which has no reason to be a constant. If $Q$ is not constant, then by adding to $P$ a multiple of $Q$ and rescaling it, we can set $P=1$, so

$$
s=\left(\begin{array}{l}
P \\
Q
\end{array}\right)=\left(\begin{array}{c}
1 \\
z-w
\end{array}\right)
$$

for some $w$. The polynomial $K=P Q^{\prime}-Q P^{\prime}$ is 1 , consistent with the absence of any 't Hooft operators. We have found, in the presence of real symmetry breaking, holomorphic data corresponding to a one-parameter family of solutions depending on the choice of a point $w \in \mathbb{R}^{2}$. This is a solution with no 't Hooft operator and a single Bethe root. We will call it a bare Miura oper. In the right context, when lifted back to four dimensions, we will interpret this solution later as a "string" that is localized at $z=w$ and at a value of $y$ that depends on $\zeta$.

In general, the degree of $K$ is at most one less than the degree of $Q$ (this bound is achieved precisely if $P=1$ ), so the number of Bethe roots is at most one more than the degree $k=\sum_{a} k_{a}$ of $K$.

\subsection{2 "Complex" symmetry breaking}

If we give expectation values at infinity to all components of $\vec{\phi}$, while requiring $A$ to vanish at infinity, then the complex connection $\mathcal{A}$ is constant and diagonal for $y \rightarrow \infty$,

$$
\begin{aligned}
& \mathcal{A}_{z} \sim \frac{1}{\zeta}\left(\begin{array}{cc}
\boldsymbol{a} & 0 \\
0 & -\boldsymbol{a}
\end{array}\right), \\
& \mathcal{A}_{\bar{z}} \sim \zeta\left(\begin{array}{cc}
\bar{a} & 0 \\
0 & -\overline{\boldsymbol{a}}
\end{array}\right), \\
& \mathcal{A}_{y} \sim\left(\begin{array}{cc}
\boldsymbol{a}_{1} & 0 \\
0 & -\boldsymbol{a}_{1}
\end{array}\right),
\end{aligned}
$$


where $\boldsymbol{a}$ is a complex number. The factors of $\zeta$ arise in the change of variables from $\varphi$ to $\mathcal{A}$.

At infinity in the $z$-direction, for any $y$, the solution reduces to the unique solution [18] of Nahm's equations which has a Nahm pole at $y=0$ and behaves as (3.32) at $y=\infty$. In particular, the connection form is constant for $z \rightarrow \infty$ with fixed $y$. For this form of the connection, we can write the small holomorphic section $s$ as

$$
s=\left(\begin{array}{cc}
\mathrm{e}^{-\zeta \overline{\boldsymbol{a}}^{\prime} \bar{z}} & 0 \\
0 & \mathrm{e}^{\zeta \overline{\boldsymbol{a}}^{\prime} \bar{z}}
\end{array}\right) s_{0}
$$

where $\boldsymbol{a}^{\prime}$ (which equals $\boldsymbol{a}$ for $y \rightarrow \infty$ ) is the constant value of $\mathcal{A}_{\bar{z}} / \zeta$ at large $z$ with fixed $y$, and $s_{0}$ has a finite limit at large $z$ and fixed $y$.

In order to analyze the holomorphic data in such solutions, it is unnatural to gauge the connection away. The information we want would be hidden in the behavior of the necessary gauge transformation at infinity. Rather, we will pick a complex gauge transformation which brings the connection exactly (not just asymptotically) to the form

$$
\begin{aligned}
& \mathcal{A}_{z}=\frac{1}{\zeta}\left(\begin{array}{cc}
\boldsymbol{a} & 0 \\
0 & -\boldsymbol{a}
\end{array}\right), \\
& \mathcal{A}_{\bar{z}}=\zeta\left(\begin{array}{cc}
\overline{\boldsymbol{a}} & 0 \\
0 & -\overline{\boldsymbol{a}}
\end{array}\right), \\
& \mathcal{A}_{y}=\left(\begin{array}{cc}
\boldsymbol{a}_{1} & 0 \\
0 & -\boldsymbol{a}_{1}
\end{array}\right) .
\end{aligned}
$$

As we will now see, this can be done by a relatively simple type of gauge transformation. Consider any gauge field on $\mathbb{R}^{2} \times \mathbb{R}_{+}$, such as one that has the asymptotic form discussed above, such that the connection form approaches a nonzero constant for $z \rightarrow \infty$ with fixed $y$. If we were to compactify $\mathbb{R}^{2}$ to $\mathbb{C P}^{1}$, we would say that such a connection has an irregular singularity at $z=\infty$ with a double pole. Connections with irregular singularities have Stokes phenomena. If one only considers gauge transformations with no essential singularities (that is, with polynomial growth only at infinity), the Stokes data is gauge-invariant. Two flat connections with an irregular singularity are gauge-equivalent by a gauge transformation with only polynomial growth at infinity if and only if they have the same monodromy and the same Stokes data.

As our connection has only has a double pole at $z=\infty$, it has only two Stokes sectors. Stokes theory tells us that the monodromy around $z=\infty$ can 
be decomposed into the product of a diagonal formal monodromy matrix, and a sequence of Stokes matrices, which are alternatingly upper and lower triangular with ones on the diagonal. With only two Stokes sectors, the expression for the monodromy is

$$
M=\left(\begin{array}{ll}
1 & b \\
0 & 1
\end{array}\right)\left(\begin{array}{ll}
1 & 0 \\
\widetilde{b} & 1
\end{array}\right)\left(\begin{array}{cc}
\mu & 0 \\
0 & \mu^{-1}
\end{array}\right),
$$

with constants $b, \widetilde{b}$, and $\mu$. As we are on $\mathbb{R}^{2}$, which is simply connected, the monodromy at infinity must be $M=1$. This together with the form (3.35) of the monodromy implies that the three factors in (3.35) - the Stokes matrices and the formal monodromy - must all equal 1. Hence, we can bring our connection to the constant diagonal form (3.34) by a gauge transformation which grows only polynomially at infinity.

In particular, $s$ will now take the form

$$
s=\left(\begin{array}{cc}
\mathrm{e}^{-\zeta \overline{\boldsymbol{a}} \bar{z}} & 0 \\
0 & \mathrm{e}^{\zeta \overline{\boldsymbol{a}} \bar{z}}
\end{array}\right)\left(\begin{array}{l}
P(z) \\
Q(z)
\end{array}\right)
$$

with polynomials $P$ and $Q$. The equation $s \wedge \mathcal{D}_{z} s=K(z)$ gives

$$
\left(P \partial_{z} Q-Q \partial_{z} P\right)-\frac{2 a P Q}{\zeta}=K(z),
$$

which can be converted to the requirement that $K \mathrm{e}^{2 \boldsymbol{a} z} / Q^{2}$ has no residues at the zeroes $w_{i}$ of $Q(z)$. This becomes

$$
\frac{2 \boldsymbol{a}}{\zeta}+\sum_{a} \frac{k_{a}}{w_{i}-z_{a}}=\sum_{j} \frac{2}{w_{i}-w_{j}} .
$$

We are interested in solutions of (3.37) modulo a rescaling $P \rightarrow \lambda P, Q \rightarrow$ $\lambda^{-1} Q$ (which corresponds to an automorphism of the diagonal flat connection), so we actually only care about the zeroes of $Q(z)$.

These are, again, Bethe equations, this time for the Gaudin model with an irregular singularity at $z=\infty$ [28-30]. It is shown in [30] that solutions of these Bethe equations are in one-to-one correspondence with eigenvectors of the spin chain, making in all $\prod_{a}\left(k_{a}+1\right)$ solutions. It may also be possible to extract this result from the theory of the XXX spin chain, which is more familiar than the Gaudin model. The Bethe equations of the Gaudin 
"ATMP-16-3-A5-GAI" — 2013/2/1 — 19:36 — page 983 — \#49

KNOT INVARIANTS

model arise from a specific "large impurity limit" of those of the XXX chain. The deformation parameter $\boldsymbol{a}$ maps to the twist of the XXX chain, which is known to break the global $S U(2)$ symmetry to $U(1)$, and simplify the counting of solutions to Bethe equations: instead of a solution for each eigenvector of the spin chain Hamiltonian that is an $S U(2)$ highest weight, one gets a solution for each eigenvector.

As a simple example, consider the case of a single 't Hooft operator of charge 2, so $K(z)=z^{2}$. Just as in Section 2.5.1, there is a solution of (3.37) with $P=1$ and $Q$ a quadratic polynomial in $z$, and a solution with $Q=1$ and $P$ a quadratic polynomial. What happens if $P$ and $Q$ are both linear in $z$ ? Again as in Section 2.5.1, we can solve (3.37) with $P=Q=z \sqrt{-\zeta / 2 a}$, but this solution does not correspond to an oper, since $P$ and $Q$ have a common zero. The novelty is that there is also an acceptable solution with $P=-(\zeta / 2 \boldsymbol{a})(z-\zeta / \boldsymbol{a}), Q=z+\zeta / \boldsymbol{a}$, corresponding to a solution of the Bethe equations (3.38) with a single Bethe root. This gives a total of $2+1=3$ opers obeying the necessary conditions. The last solution disappears if we take $\boldsymbol{a}=0$ and has a common zero for $P$ and $Q$ if we take $\zeta \rightarrow 0$.

\subsection{Opers and stress tensors}

In our analysis of opers with trivial monodromy, we have used a gauge in which the connection is trivial, $\mathcal{A}_{z}=\mathcal{A}_{\bar{z}}=0$. Correspondingly, we had to make a general ansatz for the small section $s$ that generates the holomorphic sub-bundle $L \subset E$ :

$$
s=\left(\begin{array}{l}
P \\
Q
\end{array}\right) .
$$

Here we will make a gauge transformation to put $s$ in the standard form with upper component vanishing, and see what we can say about $\mathcal{A}_{z}$. This will have two benefits. We will begin to understand the relation of opers to conformal field theory. And we will get a description that is more general, not limited to the case (which however is particularly important in the present paper) of oper bundles of trivial monodromy.

To put $s$ in a simple form by a smooth gauge transformation would make $\mathcal{A}_{z}$ and $\mathcal{A}_{\bar{z}}$ both nonzero. It turns out to be more helpful to keep $\mathcal{A}_{\bar{z}}=0$. To also keep $\mathcal{A}_{z}$ regular would require that our gauge transformation should be holomorphic, which is too restrictive a condition. Instead, we will consider meromorphic gauge transformations, which will keep $\mathcal{A}_{\bar{z}}=0$, put $s$ in a standard form, and generate poles in $\mathcal{A}_{z}$. 
The most obvious meromorphic gauge transformation that puts $s$ in a standard form is

$$
h=\left(\begin{array}{cc}
Q & -P \\
0 & Q^{-1}
\end{array}\right)
$$

We have

$$
\begin{aligned}
h s & =\left(\begin{array}{l}
0 \\
1
\end{array}\right) \\
h \partial_{z} h^{-1} & =\partial_{z}+\left(\begin{array}{cc}
-\frac{Q^{\prime}}{Q} & -K \\
0 & \frac{Q^{\prime}}{Q}
\end{array}\right),
\end{aligned}
$$

where as before $K=P Q^{\prime}-Q P^{\prime}$. It turns out to be more convenient to go to a gauge in which the upper right matrix element of $\mathcal{A}_{z}$ is -1 , at the cost of mapping $s$ to a multiple of itself (this leaves unchanged the line bundle generated by $s$ ). We make a further gauge transformation by

$$
\widetilde{h}=\left(\begin{array}{cc}
1 / \sqrt{K} & 0 \\
0 & \sqrt{K}
\end{array}\right)
$$

The possible double-valuedness of $\sqrt{K}$ is of no concern, for the following reason. If $G^{\vee}=S O(3)$, so that $G=S U(2)$, then $K$ is a perfect square as all its zeroes are of even degree. If instead $G^{\vee}=S U(2)$, then $G=S O(3)$, and we should really be writing all formulae in the adjoint representation, rather than the two-dimensional representation; accordingly, the sign of a gauge transformation is irrelevant. After a gauge transformation by $\widetilde{h}, \mathcal{A}_{z}$ takes the form

$$
\mathcal{A}_{z}=\left(\begin{array}{cc}
-v & -1 \\
0 & v
\end{array}\right)
$$

where we have set

$$
v=-\frac{K^{\prime}}{2 K}+\frac{Q^{\prime}}{Q}=-\sum_{a} \frac{k_{a} / 2}{z-z_{a}}+\sum_{i} \frac{1}{z-w_{i}} .
$$

In the last step, we used $K=\prod_{a}\left(z-z_{a}\right)^{k_{a}}, Q=\prod_{i}\left(z-w_{i}\right)$. Finally, a lower triangular gauge transformation

$$
\left(\begin{array}{ll}
1 & 0 \\
v & 1
\end{array}\right)
$$


leads to our final result for $\mathcal{A}_{z}$ :

$$
\mathcal{A}_{z}=\left(\begin{array}{cc}
0 & -1 \\
t & 0
\end{array}\right)
$$

with

$$
t=-v^{\prime}-v^{2} .
$$

In general, $t$ has poles at both the $z_{a}$ and the $w_{i}$. Near $z=z_{a}$,

$$
t \sim-\frac{j_{a}\left(j_{a}+1\right)}{\left(z-z_{a}\right)^{2}}+\frac{c_{a}}{z-z_{a}}+\cdots, j_{a}=k_{a} / 2
$$

Near $z=w_{i}$

$$
t \sim \frac{1}{z-w_{i}}\left(\sum_{a} \frac{k_{a}}{w_{i}-z_{a}}-\sum_{j \neq i} \frac{2}{w_{i}-w_{j}}\right)
$$

Thus, $t$ has no singularity at $z=w_{i}$ if and only if the Bethe equations (3.20) are satisfied.

To get some more insight, set $\mathcal{D}_{z}=\partial_{z}+\left[\mathcal{A}_{z}, \cdot\right]$ and look for a flat section, that is a holomorphic solution of $\mathcal{D}_{z}\left(\begin{array}{l}f \\ \tilde{f}\end{array}\right)=0$. We find that $\tilde{f}=f^{\prime}$ and

$$
\left(\frac{\partial^{2}}{\partial z^{2}}+t(z)\right) f=0
$$

We have carried out this derivation using a particular local coordinate $z$, but the notion that we started with - a flat bundle with an oper structure did not depend on the local coordinate. So equation (3.50) must be covariant under a change of the local coordinate, with a suitable transformation for $t$. A short calculation (or a more careful study of the above derivation) shows that under a change of local coordinate $z \rightarrow \widetilde{z}$, and a suitable transformation of $t$, the object $t$ transforms like a stress tensor in two-dimensional conformal field theory. In other words, it transforms not as a quadratic differential, as one might naively think from its pairing with the second derivative $\partial^{2} / \partial z^{2}$ in (3.50), but with an "anomalous" term involving the Schwarzian derivative $\frac{1}{2}\{z, \widetilde{z}\}$. The double pole in $t$ at $z=z_{a}$ is as if there is a primary field inserted at $z_{a}$. 
If we had started from $\mathcal{A}_{z}=\zeta^{-1} \operatorname{diag}(\boldsymbol{a},-\boldsymbol{a})$ and used the same sequence of gauge transformations, with $K=P Q^{\prime}-Q P^{\prime}-2 \boldsymbol{a} \zeta^{-1} P Q$, we would have arrived to the same formulae, but with an extra constant term in $v$ :

$$
v=-\frac{\boldsymbol{a}}{\zeta}-\sum_{a} \frac{k_{a} / 2}{z-z_{a}}+\sum_{i} \frac{1}{z-w_{i}} .
$$

Now $t(z)$ has a pole of order four at $z=\infty$. This would correspond in conformal field theory to the insertion at infinity of a somewhat unusual operator [36].

Finally, the Bethe equations have the following interesting property. They describe stationary points at fixed $z_{a}$ (and $\boldsymbol{a}$ ) of a Yang-Yang function:

$$
\begin{aligned}
\mathcal{W}\left(w_{i}, z_{a}\right)= & -\sum_{i<j} \log \left(\left(w_{i}-w_{j}\right)^{2}\right)+\sum_{i, a} k_{a} \log \left(w_{i}-z_{a}\right) \\
& -\frac{1}{4} \sum_{a<b} k_{a} k_{b} \log \left(\left(z_{a}-z_{b}\right)^{2}\right) \\
& +\frac{2 \boldsymbol{a}}{\zeta} \sum_{i} w_{i}-\frac{\boldsymbol{a}}{\zeta} \sum_{a} k_{a} z_{a} .
\end{aligned}
$$

In other words, the Bethe equations can be written as

$$
\frac{\partial \mathcal{W}}{\partial w_{i}}=0 .
$$

The Yang-Yang function has another interesting property: the coefficients of the single poles in $t$ at $z=z_{a}$ - sometimes called accessory parameters are given by

$$
c_{a}=\frac{\partial \mathcal{W}}{\partial z_{a}} .
$$

Some terms in $\mathcal{W}$ which are independent of the $w_{i}$ have been included to insure that this relation is satisfied. A similar relation holds for $a \partial_{a} \mathcal{W}$

\subsection{Opers and Virasoro conformal blocks}

In this section, we will show how these formulae arise naturally in the semiclassical limit of Virasoro conformal blocks. The semiclassical limit is defined 
as a limit in which the central charge $c$ of the Virasoro algebra goes to infinity, while the conformal dimensions of operators also scale in the same way as $c$.

There is a useful way to parameterize the central charge: $c=1+6 Q^{2}$, with $Q=b+b^{-1}$. In this parametrization, we take $b \rightarrow 0$ to get a semiclassical limit. The conformal dimensions of operators are conveniently parameterized as $\Delta=\alpha(Q-\alpha)$. The parameter $\alpha$ is often referred to as "momentum". We keep $b \alpha=\eta$ fixed as $b \rightarrow 0$. Then the insertion of an energy-momentum tensor $T$ in a correlation function scales as $b^{-2}$, and we can define the finite limit $t=b^{2} T$. We propose to identify this $t$, inserted in certain conformal blocks, with the $t$ of Section 3.6.

The quantum stress-tensor $T(z)$ has an anomaly under conformal transformations; it shifts by a multiple $\frac{c}{12}\{z, \widetilde{z}\}$ of the Schwartzian derivative. Hence $t=b^{2} T$ has a conformal anomaly that is independent of $b$ for $b \rightarrow 0$. The behavior of $t$ near $z=z_{a}$ in the previous section corresponds to the behavior near a Virasoro primary field with $\alpha_{a}=-\frac{k_{a}}{2 b}$. These operators are very special: correlation functions and conformal blocks which involve only operators of this type can be described very easily by a free-field realization. We can describe such a realization in close parallel to the discussion in the previous section.

Let $\chi$ be a two-dimensional free field with two-point function $\langle\chi(z)$ $\left.\chi\left(z^{\prime}\right)\right\rangle=-\frac{1}{2} \ln \left(z-z^{\prime}\right)$. A standard way to construct an energy-momentum tensor of central charge $c=1+6 Q^{2}$ is to take

$$
T=-: \partial \chi \partial \chi:+Q \partial^{2} \chi
$$

If we define $v=-b \partial \chi$, this definition reduces to (3.47) in the limit $b \rightarrow$ 0 . This is the first hint that a free-field realization can be useful for us. Operators of dimension $\Delta=\alpha(Q-\alpha)$ can be readily described as normalordered exponentials of the free boson,

$$
V_{\alpha}(z)=: \mathrm{e}^{2 \alpha \chi(z)}: .
$$

A second hint comes from (3.44): the quantity $v$ is the semiclassical limit of the expectation value of $-b \partial \chi$ in the presence of chiral vertex operators of momenta $-k_{a} / 2 b$ at $z=z_{a}$ and of momenta $1 / b$ at $z=w_{i}$. The operators $V_{1 / b}\left(w_{i}\right)$ have dimension 1 . They are usually called screening operators in free-field realizations [32,33], and are naturally integrated over curves. The 
singular part of the stress tensor near the location of a screening operator

$$
\begin{aligned}
& T(z) V_{1 / b}\left(w_{i}\right) \sim \frac{1}{\left(z-w_{i}\right)^{2}} V_{1 / b}\left(w_{i}\right)+\frac{1}{z-w_{i}} \partial_{w_{i}} V_{1 / b}\left(w_{i}\right)+\cdots \\
& \quad=\partial_{w_{i}}\left(\frac{1}{z-w_{i}} V_{1 / b}\left(w_{i}\right)\right) \cdots
\end{aligned}
$$

is a total derivative, and drops off upon integrating over the position of the screening operator.

We can easily compute the following free-field correlation function:

$$
\begin{aligned}
& \left\langle\prod_{i} V_{1 / b}\left(w_{i}\right) \prod_{a} V_{-k_{a} / 2 b}\left(z_{a}\right)\right\rangle_{\text {free }} \\
& \quad=\prod_{i<j}\left(w_{i}-w_{j}\right)^{-\frac{2}{b^{2}}} \prod_{i, a}\left(w_{i}-z_{a}\right)^{\frac{k a}{b^{2}}} \prod_{a<b}\left(z_{a}-z_{b}\right)^{-\frac{1}{2 b^{2}} k_{a} k_{b}} \\
& \quad=\exp \left(\frac{1}{b^{2}} \mathcal{W}(w, z)\right) .
\end{aligned}
$$

The exponent on the right is the Yang-Yang function (3.52)! (For the moment, the terms proportional to $\boldsymbol{a}$ are absent as we have not included symmetry breaking.)

Consider the integral

$$
\left\langle\prod_{a} V_{-k_{a} / 2 b}\left(z_{a}\right)\right\rangle_{\Gamma}=\int_{\Gamma}\left\langle\prod_{i} V_{1 / b}\left(w_{i}\right) \prod_{a} V_{-k_{a} / 2 b}\left(z_{a}\right)\right\rangle_{\text {free }} \prod_{i} d w_{i}
$$

where $\Gamma$ is any integration cycle for which the integral converges. Because of (3.58), this is equivalent to

$$
\left\langle\prod_{a} V_{-k_{a} / 2 b}\left(z_{a}\right)\right\rangle_{\Gamma}=\int_{\Gamma} \exp \left(\mathcal{W}\left(z_{a}, w_{i}\right) / b^{2}\right) \prod_{i} d w_{i}
$$

A Virasoro conformal block for the expectation value of a product of primary fields is a candidate correlation function that is compatible with 
“ATMP-16-3-A5-GAI" — 2013/2/1 — 19:36 — page 989 — \#55

KNOT INVARIANTS

the Virasoro Ward identity:

$$
\begin{aligned}
\left\langle T(z) \prod_{a} V_{-k_{a} / 2 b}\left(z_{a}\right)\right\rangle_{\Gamma}= & \left(\sum_{a} \frac{\Delta_{a}}{\left(z-z_{a}\right)^{2}}+\frac{1}{z-z_{a}} \frac{\partial}{\partial z_{a}}\right) \\
& \times\left\langle\prod_{a} V_{-k_{a} / 2 b}\left(z_{a}\right)\right\rangle_{\Gamma}
\end{aligned}
$$

The functions defined in (3.59) or (3.60) have this property for an arbitrary choice of the number of $w$ 's and the integration cycle $\Gamma$; this is proved using the definition (3.59) and the fact that the screening charges are primary fields of dimension 1. (The function $\left\langle T(z) \prod_{a} V_{-k_{a} / 2 b}\left(z_{a}\right)\right\rangle_{\Gamma}$ is defined by the integral (3.59) with an insertion of $T(z)$ in the free-field correlation function on the right-hand side.) What we have just described is the freefield realization of the conformal blocks $[32,33]$.

The space of possible integration cycles $\Gamma$ for the integral (3.60) has a natural flat connection (the Gauss-Manin connection) as the points $z_{a}, a=$ $1, \ldots, d$ vary. Hence the functions $\left\langle\prod_{a} V_{-k_{a} / 2 b}\left(z_{a}\right)\right\rangle_{\Gamma}$ - for any fixed number of w's - furnish a representation of the braid group on $d$ strands. It is known $[34,35]$ that these are precisely the representations of the braid group that are associated to the Jones polynomial and its generalizations. This relation of the Jones polynomial to conformal field theory will be more useful for the present paper than the relation via the Knizhnik-Zamolodchikov equation, which was noted in Section 3.4.1.

The functions $\left\langle\prod_{a} V_{-k_{a} / 2 b}\left(z_{a}\right)\right\rangle_{\Gamma}$ are not all possible conformal blocks for a product of primary fields with the dimensions of the $V_{-k_{a} / 2 b}$; rather, they are all such conformal blocks if the operators $V_{-k_{a} / 2 b}$ are degenerate primary fields in the sense introduced in [31]. Alternatively, these functions are all possible conformal blocks if the oper derived from the small $b$ limit of $t=b^{2} T$ is supposed to have trivial monodromy at the points $z=z_{a}$. These concepts and the relation between them are described in Section 3.7.2.

\subsubsection{The irregular case}

Now let us incorporate the complex symmetry breaking parameter $\boldsymbol{a}$ in the above discussion. We can certainly in the integral (3.60) over the $w$ 's modify the exponent to include the terms proportional to $\boldsymbol{a}$ in the YangYang function (3.52). But what does this mean in conformal field theory? 
We need to replace the free-field correlation function (3.58) by

$$
\begin{aligned}
& \left\langle\prod_{i} V_{1 / b}\left(w_{i}\right) \prod_{a} V_{-k_{a} / 2 b}\left(z_{a}\right)\right\rangle_{\text {free }}=\prod_{i \neq j}\left(w_{i}-w_{j}\right)^{-\frac{2}{b^{2}}} \prod_{i, a}\left(w_{i}-z_{a}\right)^{\frac{k_{a}}{b^{2}}} \\
& \quad \times \prod_{a \neq b}\left(z_{a}-z_{b}\right)^{-\frac{1}{b^{2}} k_{a} k_{b}} \mathrm{e}^{\frac{a}{\zeta b^{2}}\left(2 \sum_{i} w_{i}-\sum_{a} z_{a}\right)}
\end{aligned}
$$

What is the conformal field theory interpretation of this formula?

Almost by construction, the right-hand side is the free-field correlation function of the given product of fields with peculiar boundary conditions for $\chi$ at infinity, $\chi \sim \boldsymbol{a} z / b \zeta$. Alternatively, we have inserted at infinity an "irregular vertex operator", i.e., the $L \rightarrow \infty$ limit of

$$
\exp \left(-\frac{2 a}{\zeta b} L^{2} \partial \chi(L)\right) \text {. }
$$

In the presence of such an irregular vertex operator, the stress-tensor has the expected degree four pole. Nothing changes in the above formulae, except that the choice of possible integration contours is enlarged. The result of the integral is a conformal block with an irregular puncture at infinity, as defined in [36], in addition to the standard punctures of momenta $-k_{a} / 2 b$. In the context of free fermions, operators associated to irregular singularities were originally defined in $[37,38]$.

\subsubsection{Degenerate primary fields and trivial monodromy}

Now we will review some standard facts about representations of the Virasoro algebra. This will enable us to explain what is special about the particular conformal blocks that come from the free-field representation.

For a generic value of the conformal dimension $\Delta$, the Verma module defined as the span of all possible Virasoro descendants of a highest weight vector of dimension $\Delta$ is irreducible. For a set of special values

$$
\alpha=\alpha_{r, s}=-\frac{(r-1) b}{2}-\frac{s-1}{2 b}, \quad r, s=1,2,3, \ldots
$$

or $\alpha=Q-\alpha_{r, s}$, this is not true: a certain descendant at level $r s$ is again a highest weight vector, and has zero norm. In a unitary conformal field theory, the descendant in question will vanish, and even in a nonunitary theory, it might vanish. The primary field whose descendant vanishes is called a degenerate primary field. We write $\Phi_{r, s}$ for such a field. 
"ATMP-16-3-A5-GAI" — 2013/2/1 — 19:36 — page 991 — \#57

KNOT INVARIANTS

The vanishing descendant of a degenerate primary field will certainly decouple in correlation functions. We call conformal blocks obeying such a relation degenerate conformal blocks. They satisfy a condition known as a "degenerate fusion rule". (It can be proved using the differential equation that we mention shortly.) In the Operator Product Expansion (OPE) of an operator $\Phi_{r, s}$ and an operator of momentum $\alpha$, only operators of momentum $\alpha-\frac{r^{\prime} b}{2}-\frac{s^{\prime}}{2 b}$ appear, with

$$
r^{\prime}=r-1, r-3, \ldots, 1-r \quad s^{\prime}=s-1, \quad s-2 \ldots, 1-s .
$$

A conformal block with an insertion of momentum $\alpha_{r, s}$ satisfies the nullvector decoupling condition if and only if its correlation functions satisfy a certain differential equation of order $r s$. An important special case is $r=2$, $s=1$; the equation is

$$
\partial^{2} \Phi_{2,1}(z)+b^{2}: T(z) \Phi_{2,1}(z):=0 .
$$

This reduces in the semiclassical limit to the differential equation (3.50) associated to an oper. (For $b \rightarrow 0$, the normal ordering in (3.66) is irrelevant; the only part of $b^{2} T$ that survives for $b \rightarrow 0$ is the response to the "heavy" fields with momenta of order $1 / b$.) Moreover, in the semiclassical limit, the expectation value of $t=b^{2} T$ will always be such that the monodromy of the differential equation around points with additional $\Phi_{1, s}$ insertions is trivial. Indeed, the degenerate fusion rule implies that the OPE of $\Phi_{2,1}$ and $\Phi_{1, s}$ contains only one primary field $\Phi_{2, s}$; from this, it follows that the monodromy of $\Phi_{2,1}$ around a $\Phi_{1, s}$ puncture is trivial. This is a quantum version of the trivial monodromy condition on the differential equation (3.50) associated to the oper. On the other hand, the singularity of $t$ near a $\Phi_{1, s}$ insertion is precisely that which we have exhibited in (3.48) (with $j=s / 2$ ). The upshot of this is that the semiclassical limit of a conformal block for a correlation function $\left\langle\prod_{a=1}^{d} \Phi_{1, k_{a}}\left(z_{a}\right)\right\rangle$ determines an oper with precisely the sort of monodromy-free singularities that we extracted from three-dimensional gauge theory in Section 3.3.

The conformal blocks constructed from the free-field formula (3.59) describe correlation functions of degenerate primary fields, simply because the free-field vertex operators of momenta $-\frac{k_{a}}{2 b}$ are degenerate. Related to this, it is possible to show that the conformal blocks which are given by the free-field realization do satisfy the degenerate fusion constraints. The corresponding differential equations are equivalent to the Picard-Fuchs equations satisfied by the free-field integrals, or to the natural flat connection on 


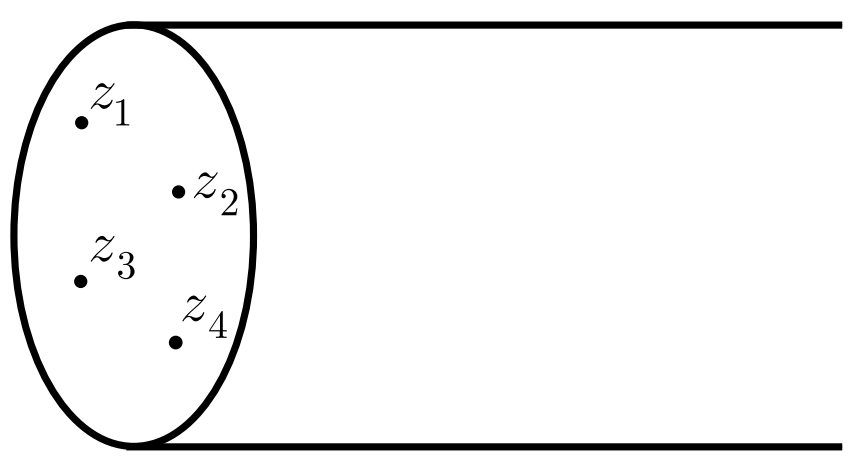

Figure 6: A snapshot at fixed time of a time-independent situation. In the three-manifold $M_{3}=C \times \mathbb{R}_{+}$, knots are present at boundary points $z_{1}, \ldots, z_{4}$.

the space of integration cycles. They are a close analog to the KnizhnikZamolodchikov equations. The free-field realization gives all the conformal blocks for the correlation function $\left\langle\prod_{a=1}^{d} \Phi_{1, k_{a}}\left(z_{a}\right)\right\rangle$ that are allowed by the fusion rules, so there are no more to be had.

The interpretation of opers with monodromy-free singularities in terms of correlation functions of degenerate conformal fields gives an intuitive explanation to the Bethe equations. Naturally, $t$ should have no poles at the points $w_{i}$, because no conformal fields are inserted there.

\section{Four-dimensional solutions and parallel transport}

\subsection{Introduction}

We now turn to the problem of analyzing time-dependent solutions of the original BPS equations (1.1). Even though this is a problem of classical PDEs, a quantum mechanical view is helpful.

We start with a time-independent situation - twisted $\mathcal{N}=4$ super YangMills on a four-manifold $M_{4}=\mathbb{R} \times M_{3}$, where $M_{3}$ is a three-manifold and we think of $\mathbb{R}$ as the time direction. In our application, $M_{3}=C \times \mathbb{R}_{+}$, with $C$ a Riemann surface. If knots are present, we assume initially that they are time-independent. In this situation, which is depicted again for convenience in figure 6 , we want to find the BPS states - quantum ground states.

The first approximation, already analyzed in Sections 2 and 3, is to find time-independent classical solutions. In going from classical solutions to 
"ATMP-16-3-A5-GAI" — 2013/2/1 — 19:36 — page 993 — \#59

KNOT INVARIANTS

BPS states, we will ignore the noncompactness of $M_{3}$. This means that we will ignore the existence of a continuum of non-BPS excitations.

If there is only a finite set of classical solutions of the BPS equations (and they are nondegenerate - there are no zero modes in expanding around such a solution), then the classical approximation to the space of BPS states is very simple. Let $\mathcal{I}$ be the set of classical solutions. Then for each $I \in \mathcal{I}$, there is in perturbation theory a quantum ground state $\psi_{I}$ that is localized near $I$. In the perturbation theory, the $\psi_{I}$ form a basis for the space $\mathcal{H}$ of BPS states.

Nonperturbatively, in problems of this general type, instanton effects might lift some of these approximate ground states away from zero energy. However, we are actually here dealing with a problem in which this does not occur. This is because in the time-independent case, even with knots present, our problem has four supercharges, and an instanton (that is, a classical solution with nontrivial time dependence) violates at least two of them. This leads to the existence of two fermion zero modes in an instanton background, which is one too many to contribute to a matrix element of the supercharge $Q$ between approximate ground states $\psi_{I}$ and $\psi_{J}$.

\subsubsection{Relation to Morse theory}

A more explicit understanding of why instantons do not lift the classical vacuum degeneracies comes from the relation of this problem to Morse theory. ${ }^{8}$ Supersymmetric quantum mechanics related to Morse theory [42] is, in general, a theory of maps from $\mathbb{R}$ to $\mathcal{U}$, where $\mathcal{U}$ is a Riemannian manifold with metric tensor $g$ endowed with a real-valued function $h$ that we call the superpotential. For generic $\mathcal{U}$ and $h$, the model has two supercharges, one of which is conjugate to the exterior derivative:

$$
Q=\mathrm{e}^{h} d \mathrm{e}^{-h} .
$$

The classical vacua correspond to critical points of $h$. If $h$ is a Morse function - that is, its critical points are all isolated and nondegenerate - then in perturbation theory, each critical point $I$ corresponds to an approximate quantum ground state $\psi_{I}$. The fermion number $q_{I}$ of $\psi_{I}$ is equal to the Morse index of the critical point $I$ (the number of negative eigenvalues of the matrix of second derivatives of the function $h$ at $I$ ). Since $Q$ increases the fermion number by one unit, quantum corrections inducing nonzero matrix elements $\left\langle\psi_{J}|Q| \psi_{I}\right\rangle$ are possible only if

$$
q_{J}=q_{I}+1 .
$$

\footnotetext{
${ }^{8}$ For a relatively accessible introduction to the relevant aspects of Morse theory, see [39].
} 
Such nonzero matrix elements can be computed by counting, in a suitable sense, the instanton solutions that interpolate between the critical point $I$ in the far past and the critical point $J$ in the far future. The relevant "instanton" equations, in other words the conditions for a map $\mathbb{R} \rightarrow \mathcal{U}$ to be $Q$-invariant, are the gradient flow equations of Morse theory:

$$
\frac{d x^{i}}{d t}=-g^{i j} \frac{\partial h}{\partial x^{j}}
$$

The problem we are studying of twisted $\mathcal{N}=4$ super Yang-Mills theory on $\mathbb{R} \times M_{3}$ (we primarily take $M_{3}=C \times \mathbb{R}_{+}$but the following remarks are more general) is an infinite-dimensional problem of this sort, ${ }^{9}$ with $\mathcal{U}$ being the space of complex-valued connections on $M_{3}$. We view the Chern-Simons function

$$
\mathcal{W}=\frac{1}{4 \pi \mathrm{i}} \int_{M_{3}} \operatorname{Tr}\left(\mathcal{A} \wedge d \mathcal{A}+\frac{2}{3} \mathcal{A} \wedge \mathcal{A} \wedge \mathcal{A}\right)
$$

as a holomorphic function on the complex manifold $\mathcal{U}$. Holomorphy means that the one-dimensional sigma model with target $\mathcal{U}$ and superpotential $\mathcal{W}$ has four supercharges (this actually depends on the fact that the metric of $\mathcal{U}$ is Kahler and is also true in the gauge-invariant case mentioned in footnote $9)$. We actually want to study this model in the context of a twisting of $\mathcal{N}=4$ super Yang-Mills theory in which a particular supercharge $Q$ is distinguished. This supercharge depends on a twisting parameter $t$ [15] and is an infinite-dimensional version of $Q=\mathrm{e}^{h} d \mathrm{e}^{-h}$ where $h$ is the ordinary Morse function

$$
h=\operatorname{Re}\left(\mathrm{e}^{\mathrm{i} \alpha} \mathcal{W}\right),
$$

and

$$
t=\frac{1-\sin \alpha}{\cos \alpha}
$$

\footnotetext{
9 To be more precise, our problem is a gauge-invariant version of a such a problem corresponding to a supersymmetric sigma-model with target $\mathcal{U}$ coupled to gauge fields that gauge a symmetry of $\mathcal{U}$. The gauge group in our case is the group of maps from $M_{3}$ to the finite-dimensional group $G$, while $\mathcal{U}$ is the space of complex-valued connections on $M_{3}$. However, in our problem the gauge group acts freely on $\mathcal{U}$; this is ensured by the Nahm pole boundary condition. As a result, the gauge-invariance will not play a major role. In effect, for our purposes, we can replace $\mathcal{U}$ by its quotient by the group of complex gauge transformations and reduce to the case that there are no gauge fields.
} 
"ATMP-16-3-A5-GAI" — 2013/2/1 — 19:36 — page 995 — \#61

KNOT INVARIANTS

For a Morse function of this type, the gradient flow equation becomes

$$
\frac{d \bar{w}^{j}}{d t}=-g^{\bar{j} i} \frac{\mathrm{e}^{\mathrm{i} \alpha}}{2} \frac{\partial \mathcal{W}}{\partial w^{i}},
$$

where the $w^{i}$ are local holomorphic coordinates on $\mathcal{U}$.

A down-to-earth manifestation of the relation of our problem to Morse theory is that the underlying four-dimensional supersymmetric equations (1.1) are the gradient flow equations (4.3) for the Morse function $h$ on the infinite-dimensional manifold $\mathcal{U}$. (This is one of the main ideas in [40,41], where the gauge invariance mentioned in footnote 9 has been taken into account.)

Now we can give a more explicit explanation of why nonperturbative effects in our problem will not spoil the supersymmetry of any of the approximate quantum ground states $\psi_{I}$. In general, for a Morse function that is the real part of a holomorphic function, isolated critical points all have the same (middle-dimensional) Morse index and thus the same value of the fermion number $q$. Hence the condition (4.2) is never satisfied.

Another route to the same result is as follows. In general, gradient flow for a Morse function such as $h$ that is the real part of a holomorphic function has a conserved quantity, namely the imaginary part of the relevant holomorphic function, in our case

$$
j=\operatorname{Im}\left(\mathrm{e}^{\mathrm{i} \alpha} \mathcal{W}\right) .
$$

For generic $\alpha$, all critical points have distinct values of $j$ and hence distinct critical points cannot be connected by a solution of the gradient flow equation. Hence there are no instantons that might spoil the supersymmetry of the states $\psi_{I}$.

\subsubsection{Time dependence}

As explained in Section 1.2, we do not literally want to consider a timeindependent situation; rather, we want to allow for a slow time-dependence of the positions of the knots. Let $\mathcal{B}$ be the space of distinct points $z_{1}, \ldots, z_{d} \in$ $C$. The space $\mathcal{H}$ of BPS states is the fiber of a bundle $\widehat{\mathcal{H}}$ over $\mathcal{B}$. This bundle carries a natural flat connection. This is a general property of topological field theory, but the Morse theory interpretation leads to a particularly nice description.

In supersymmetric quantum mechanics related to Morse theory, since the supercharge $Q=e^{h} d e^{-h}$ is conjugate to the exterior derivative $d$, the ground 
“ATMP-16-3-A5-GAI" — 2013/2/1 — 19:36 — page 996 — \#62

states $\psi_{I}$ associated to critical points must have an interpretation in terms of the cohomology or dually the homology of $\mathcal{U}$. There is a standard way to understand this in Morse theory. To a critical point $I$, one associates the downward-flowing cycle $\mathcal{J}_{I}$ consisting of all points in $\mathcal{U}$ that can be reached by gradient flow starting at $I$. In other words, one considers solutions of the gradient flow equation on a half-line $(-\infty, 0]$, with initial conditions that the flow starts at $I$ at $t=-\infty$. $\mathcal{J}_{I}$ parameterizes the values at $t=0$ of such flows.

The case that $h$ is the real part of a holomorphic function has special features, and is particularly simple, so let us focus on that case. We make the further simplifying assumption that the critical points of $h$ are isolated and irreducible. $\mathcal{U}$ is inevitably not compact (or it would not admit a nonconstant holomorphic function). In this very special situation, the $\mathcal{J}_{I}$ are called Lefschetz thimbles. The thimbles $\mathcal{J}_{I}$ are closed if the angle $\alpha$ used in defining $h$ is sufficiently generic (to prevent the existence of gradient flows between distinct critical points), but they are not compact. So they do not represent classes in the ordinary homology of $\mathcal{U}$. However, as $\mathcal{J}_{I}$ is defined by downward gradient flow with respect to $h$, one has $h \rightarrow-\infty$ at infinity along $\mathcal{J}_{I}$. As a result, the $\mathcal{J}_{I}$ are elements of a certain relative homology group - the homology $H\left(\mathcal{U}, \mathcal{U}_{<}\right)$of $\mathcal{U}$ relative to the region $\mathcal{U}_{<}$ where $h$ goes to $-\infty$. (In the notation, we do not indicate the dimension of a homology cycle, because this relative homology is nonzero only in the middle dimension. That is related to the fact that the critical points all have a middle-dimensional Morse index.)

The space $\widehat{\mathcal{H}}$ of supersymmetric ground states can be identified with the relative homology $H\left(\mathcal{U}, \mathcal{U}_{<}\right)$. In this correspondence, the quantum ground state $\psi_{I}$ associated to a critical point $I$ maps to the element $\mathcal{J}_{I}$ of $H\left(\mathcal{U}, \mathcal{U}_{<}\right)$. For an explanation of this from a physical point of view (in the context of supersymmetric quantum mechanics related to Morse theory); see [41,43].

The interpretation in terms of relative homology means that $\widehat{\mathcal{H}}$ has an integral structure and hence a natural flat connection. To give it a fancy name, this flat connection is the Gauss-Manin connection on the relative homology. This connection is trivial for generic values of $\alpha$ and the $z_{i}$ : the $\psi_{I}$ are flat sections, and the connection on $\widehat{\mathcal{H}}$ is fully described by the smooth evolution of the classical critical points and corresponding thimbles. (In transporting the thimbles, one must keep track of their orientations; the sign of the relative homology class associated to $\mathcal{J}_{I}$ depends on the orientation of $\mathcal{J}_{I}$.)

Crucially, there are codimension one walls in the space $S^{1} \times \mathcal{B}$ of parameters $\alpha$ and $z_{i}$ where the thimbles fail to be closed (and so do not define 
elements of the relative homology), and the map from the critical points to quantum states jumps discontinuously. This can occur if there are gradient flow lines from $I$ to $J$; in this case, $\mathcal{J}_{I}$ is not closed as it contains points arbitrarily close to $J$, but not $J$ itself.

We write $\ell_{I}^{J}$ for the locus in $S^{1} \times \mathcal{B}$ on which the following necessary conditions are obeyed for flows from $I$ to $J$ : the value of the conserved quantity $j$ is equal at $I$ and $J$, while $h(I)>h(J)$. The first condition is a single real condition, while the second is just an inequality. So $\ell_{I}^{J}$ is of real codimension 1, and we call it a Stokes wall.

In crossing a Stokes wall $\ell_{I}^{J}$, only the thimble $\mathcal{J}_{I}$ becomes ill-defined. It jumps by a multiple of $\mathcal{J}_{J}$ :

$$
\mathcal{J}_{I} \rightarrow \mathcal{J}_{I}+\mathfrak{m}_{I J} \mathcal{J}_{J}
$$

where $\mathfrak{m}_{I J}$ is the "number" of gradient flow lines from $I$ to $J$ counted in an appropriate sense. A given line contributes 1 or -1 to the sign depending on the direction in which the difference between the values of $j=\operatorname{Im}\left(\mathrm{e}^{\mathrm{i} \alpha} \mathcal{W}(w, z)\right)$ at $I$ and $J$ passes through zero. For an elementary explanation of such matters, see Section 2 of [40].

The correspondence between states $\psi_{I}$ and thimbles $\mathcal{J}_{I}$ means that the $\psi_{I}$ have the same jumping in crossing Stokes walls. In the $\psi_{I}$ basis, the connection is trivial except across the Stokes walls, where the transport matrix is a triangular "Stokes factor"

$$
S\left[\ell_{I}^{J}\right]=1+\mathfrak{m}_{I J} \mathrm{e}_{I}^{J}
$$

Here $\mathrm{e}^{J}{ }_{I}$ is the matrix whose only nonzero element is 1 at position $J, I$.

In particular, the parallel transport along a path $\mathcal{P}$ in $S^{1} \times \mathcal{B}$ is a pathordered product of factors of the following kind: (a) between two Stokes walls, one has only the "formal monodromy" which expresses the permutations of the classical critical points, with minus signs that keep track of the orientations of the thimbles; (b) every time one crosses a Stokes wall, the monodromy acquires a corresponding Stokes factor.

One can visualize the matrix elements $\mathfrak{n}_{I}^{J}[\mathcal{P}]$ of the transport matrix for the path $\mathcal{P}$ as counting paths from a critical point $I$ to a critical point $J$, where away from Stokes walls, one has to follow a critical point continuously, but in crossing a wall, one is allowed to "jump" along a gradient flow trajectory from one critical point to the next. A matrix element of the transport matrix is given by a sum of contributions of hybrid paths of this 
type, with each path contributing 1 or -1 depending on how the orientation of a thimble evolves along the given path.

Incidentally, the fact that the monodromy for parallel transport along a path $\mathcal{P}$ depends only on the homotopy class of $\mathcal{P}$ implies wall-crossing formulae for the numbers $\mathfrak{m}_{I J}$ that control the jumping. More generally, the whole picture can be interpreted in terms of BPS states in an LG model based on $\mathcal{W}$, but we will not pursue that interpretation in this paper.

Let us collect a few properties of the matrix elements $\mathfrak{n}_{I}^{J}[\mathcal{P}]$ :

- $\mathfrak{n}_{I}^{J}[\mathcal{P}]$ only depends on the homotopy class of $\mathcal{P}$;

- The composition of paths maps to matrix multiplication: $\mathfrak{n}_{I}^{K}\left[\mathcal{P}_{1} \circ\right.$ $\left.\mathcal{P}_{2}\right]=\sum_{J} \mathfrak{n}_{I}^{J}\left[\mathcal{P}_{1}\right] \mathfrak{n}_{J}^{K}\left[\mathcal{P}_{2}\right]$

- For an infinitesimal path $\delta \mathcal{P}$ from $z$ to $z+\delta z$ it is almost always true that $\mathfrak{n}_{I}^{J}[\delta \mathcal{P}]=\delta_{I}^{J}$ - this fails only in crossing a Stokes wall;

- In crossing a Stokes wall, $\mathfrak{n}_{I}^{J}[\delta \mathcal{P}]-\delta_{I}^{J}=\mathfrak{m}_{I J}$, where $\mathfrak{m}_{I J}$ is computed by a count of flow lines.

\subsubsection{The dual basis}

If $\Gamma$ is any cycle in the relative homology $H\left(\mathcal{U}, \mathcal{U}_{<}\right)$, then as the thimbles are a basis for this relative homology, $\Gamma$ is equivalent in relative homology to a linear combination of thimbles,

$$
\Gamma=\sum_{I} \mathfrak{c}^{I} \mathcal{J}_{I}
$$

How can the coefficients $\mathfrak{c}^{I}$ be determined? If we had, in some sense, a dual basis $\mathcal{K}^{I}$ to the $\mathcal{J}_{I}$ 's with pairings

$$
\left\langle\mathcal{K}^{J}, \mathcal{J}_{I}\right\rangle=\delta^{J}{ }_{I},
$$

then we would identify the coefficients in (4.11) as

$$
\mathfrak{c}^{I}=\left\langle\mathcal{K}^{I}, \Gamma\right\rangle
$$

The Poincaré dual of the relative homology $H\left(\mathcal{U}, \mathcal{U}_{<}\right)$is the opposite relative homology $H\left(\mathcal{U}, \mathcal{U}_{>}\right)$of $\mathcal{U}$ relative to the region $\mathcal{U}_{>}$with $h \rightarrow+\infty$. A natural basis of $H\left(\mathcal{U}, \mathcal{U}_{>}\right)$is given by the upward-flowing thimble $\mathcal{K}^{I}$ associated to critical points. For each critical point $I, \mathcal{K}^{I}$ is defined as the boundary values at $t=0$ of solutions of the gradient flow equation on the half-line $[0, \infty)$ that approach the point $I$ for $t \rightarrow+\infty$. Since $h$ decreases 
"ATMP-16-3-A5-GAI" — 2013/2/1 — 19:36 — page 999 — \#65

KNOT INVARIANTS

along gradient flow lines, the smallest value it assumes along such a flow is its value at $t=\infty$, which is its value at the critical point $I$. So $h$ is bounded below along $\mathcal{K}^{I}$, but possibly not bounded above, and $\mathcal{K}^{I}$ takes values in $H\left(\mathcal{U}, \mathcal{U}_{>}\right)$. As for the pairing (4.12), from the definitions of the $\mathcal{K}$ 's and $\mathcal{J}^{\prime}$ 's, $\left\langle\mathcal{K}^{J}, \mathcal{J}_{I}\right\rangle$ counts flows on the full real line $(-\infty, \infty)$ that start at $J$ at $t=-\infty$ and end at $I$ at $t=+\infty$. For $J \neq I$ there are no such flows (for generic $\alpha$ where the thimbles are well-defined). For $J=I$, since $h$ strictly decreases along a nonconstant flow, the only flow is the constant one that sits at $J$ for all times. For a certain natural relative orientation of the $\mathcal{J}$ 's and $\mathcal{K}$ 's, the contribution of the constant flow to the pairing is +1 .

\subsubsection{Non-single valued superpotentials and $q$-grading}

In the framework that we have presented so far, the matrices which represent the action of the braid group on $\mathcal{H}$ have integer-valued entries, since the relative homology has an integral structure. Now we want to consider a situation where $\mathcal{W}$ is not single-valued. To be more precise, we will consider a holomorphic function like the Chern-Simons functional $\mathcal{W}$, whose real part is single-valued, but whose imaginary part is well defined only modulo $2 \pi \mathbb{Z}$. We can reduce to the framework which we have employed so far by replacing $\mathcal{U}$ by the smallest cover $\widehat{\mathcal{U}}$ on which $\mathcal{W}$ is single-valued.

Passing to $\widehat{\mathcal{U}}$ comes at the cost that now the number of critical points will be infinite, since each critical point in $\mathcal{U}$ has infinitely many preimages in $\widehat{\mathcal{U}}$. A critical point $\widehat{I}$ in $\widehat{\mathcal{U}}$ is the same as a critical point $I$ in $\mathcal{U}$ together with a choice of a branch of $\mathcal{W}$ at $I$. Locally, we can pick an arbitrary preimage $\widehat{I}_{0}$ of a critical point $I$, and denote as $\widehat{I}_{n}$ the critical point for which the value of $\mathcal{W}$ is shifted by $-2 \pi \mathrm{i} n$ compared to the value at $\widehat{I}_{0}$.

The Stokes factors and transport matrices are now matrices of infinite size, but their matrix elements can be computed by the techniques we have described, and are integer-valued. As the calculations only depend on the gradient of $\mathcal{W}$, these matrices commute with the deck transformation $\widehat{I}_{n} \rightarrow \widehat{I}_{n+1}$. It is convenient to introduce a variable $q$ taking values in $\mathbb{C}^{*}$. Then if we simply write $q^{n} \psi_{I}$ as a symbolic shorthand for $\psi_{\widehat{I}_{n}}$, we can replace infinite-dimensional matrices whose entries are integers with finitedimensional matrices whose entries are Laurent polynomials in $q$ with integer coefficients. We denote such a matrix as $S\left[\ell_{I}^{J} ; q\right]$. In order to keep track of the lift of $\mathcal{W}$ as we move in $\mathcal{B}$, the formal monodromy matrices which encode the permutation of critical points and the Stokes matrices associated to gradient flows are valued in powers of $q$, to keep track of the change of $\mathcal{W}$ along a path. The matrix elements of the transport matrix are now obtained by summing over hybrid paths (continuous evolution away from Stokes walls 
and gradient flow across Stokes walls) with weight $\pm q^{n}$, where $-2 \pi \mathrm{i} n$ is the change of superpotential along the path and as usual the sign involves the orientation of the thimbles.

An alternative description of all this is as follows. Since $\mathcal{U}$ admits the nonsingle-valued superpotential $\mathcal{W}$, its first Betti number is positive and one can introduce a "theta-angle" $\theta$ in the supersymmetric quantum mechanics, weighting by $\mathrm{e}^{\mathrm{i} n \theta}$ a path in which $\mathcal{W}$ jumps by $-2 \pi \mathrm{i} n$. This has the effect of replacing the relative homology of $\mathcal{U}$ with a twisted version of the relative homology, valued in a flat line bundle of monodromy $q=\mathrm{e}^{\mathrm{i} \theta}$. Then we consider the Gauss-Manin connection for homology twisted by this flat bundle. The holonomy matrices for this connection have entries that are Laurent polynomials in $q$ with integer coefficients and they can be computed as just described.

\subsection{Classical description of counting of four-dimensional solutions}

Now, let us consider the problem that we are really interested in - timedependent solutions of the supersymmetric equations (1.1) on $\mathbb{R} \times C \times \mathbb{R}_{+}$. The solutions will be time-dependent because the boundary conditions are time-dependent - we allow the positions $z_{i}$ of singular monopoles on the boundary of $M_{3}=C \times \mathbb{R}_{+}$to vary with time. Although we typically assume an adiabatic evolution of the monopole positions, the counting of fourdimensional solutions is topological, and the adiabatic assumption is not necessary.

In the simplest setup, with singular monopole strands at the boundary braided in time, the superpotential depends holomorphically on some parameters (the positions of the strands in $C$ ), and the parameters evolve in time. Schematically, the equations take the form of a "forced gradient flow"

$$
\frac{d \bar{w}_{\bar{i}}}{d t}=-\mathrm{e}^{\mathrm{i} \alpha} g^{\bar{i} j} \frac{\partial \mathcal{W}(w, z(t))}{\partial w_{j}} .
$$

We suppose that the singular monopoles begin at positions $\vec{z}_{i}=$ $\left(z_{1}, \ldots, z_{k}\right)$ near time $t=-\infty$ and end at positions $\vec{z}_{f}=\left(z_{1}^{\prime}, \ldots, z_{k}^{\prime}\right)$ near $t=+\infty$. In fact, we can assume that the positions $z_{i}(t)$ of the singular monopoles are constant except in a bounded interval $-T<t<T$, for some $T$, during which they follow a path $\mathcal{P}$ in their parameter space $\mathcal{B}$. In such a situation, we can look for solutions of the forced gradient flow equation that begin at a specified critical point $I$ of $\mathcal{W}\left(w, \vec{z}_{i}\right)$, and end at a specified critical point $J$ of $\mathcal{W}\left(w, \vec{z}_{f}\right)$. The "number" of such solutions, with each 
solution weighted by the sign of the fermion determinant, is a topological invariant - unchanged under deformations of the path $\mathcal{P}$ or the metric on $\mathcal{U}$. We will call this invariant $\mathfrak{N}_{I}^{J}$. (For a reason that will be clear momentarily, we really only want to define $\mathfrak{N}_{I}^{J}$ if $\vec{z}_{i}$ and $\vec{z}_{f}$ are not on Stokes walls.)

In Section 4.1.2, we already associated an integer invariant $\mathfrak{n}_{I}^{J}[\mathcal{P}]$ to this situation. $\mathfrak{n}_{I}^{J}[\mathcal{P}]$ was a matrix element of the Gauss-Manin connection for transport along the path $\mathcal{P}$ from $\vec{z}_{i}$ to $\vec{z}_{f}$. One can think of $\mathfrak{n}_{I}^{J}[\mathcal{P}]$ as the expansion coefficients when a thimble $\mathcal{J}_{I}$ in the relative homology $H\left(\mathcal{U}, \mathcal{U}_{<}\right)_{\vec{z}_{i}}$ is transported along the path $\mathcal{P}$ using the Gauss-Manin connection, and then expressed in terms of the thimbles $\mathcal{J}_{J}^{\prime}$ that furnish a basis of $H\left(\mathcal{U}, \mathcal{U}_{<}\right)_{\vec{z}_{f}}$ :

$$
\mathcal{J}_{I}=\sum_{J} \mathfrak{n}_{I}^{J} \mathcal{J}_{J}^{\prime}
$$

We claim that in fact

$$
\mathfrak{N}_{I}^{J}=\mathfrak{n}_{I}^{J}
$$

The importance of this relation is that $\mathfrak{N}_{I}^{J}$ is what we want, the counting of time-dependent solutions, while $\mathfrak{n}_{I}^{J}$ is more easily computed, since this requires only the study of time-independent problems.

One explanation of (4.16) is as follows. By definition, $\mathcal{J}_{I}\left[\vec{z}_{i}\right]$ is the set of points in $\mathcal{U}$, which can be reached by flows

$$
\frac{d \bar{w}_{\bar{i}}}{d t}=-\mathrm{e}^{\mathrm{i} \alpha} g^{\bar{i} j} \frac{\partial \mathcal{W}\left(w, z_{i}\right)}{\partial w_{j}}
$$

which asymptote to $I$ in the past. Here the $z_{i}$ are regarded as constants. Now consider equation (4.14) for forced gradient flow on the semi-infinite interval $\left(-\infty, t_{0}\right]$. For $t_{0} \leq-T$, the values at $t_{0}$ of a solution of this equation parameterize $\mathcal{J}_{I}\left[\vec{z}_{i}\right]$, but for $t_{0}>-T$, they parameterize a $t_{0}$-dependent continuous deformation of this space that we will call $\widetilde{\mathcal{J}}_{I}\left[\vec{z}_{i} ; t_{0}\right]$.

We saw in Section 4.1.3 that that for any given cycle $\Gamma$ in $H_{m}\left(\mathcal{U}, \mathcal{U}_{<}\right)_{\vec{z}_{f}}$, the coefficients $\mathfrak{c}^{J}$ in the expansion

$$
\Gamma=\sum_{J} \mathfrak{c}^{J} \mathcal{J}_{J}\left[\vec{z}_{f}\right]
$$


count, in the sense of an index, the number of flows

$$
\frac{d \bar{w}_{\bar{i}}}{d t}=-\mathrm{e}^{\mathrm{i} \alpha} g^{\bar{i} j} \frac{\partial \mathcal{W}\left(w, z_{i}\right)}{\partial w_{j}}
$$

which start from $\Gamma$ and asymptote to the critical point $J$ in the future.

Hence, the number of four-dimensional solutions which flow from $\widetilde{\mathcal{J}}_{I}\left[\vec{z}_{i} ; t_{0}\right]$ at some given time $t_{0}$ after the braiding occurs to the critical point $J$ in the future are the coefficients $\widetilde{\mathfrak{N}}_{I}^{J}$ in the expansion

$$
\widetilde{\mathcal{J}}_{I}\left[\vec{z}_{i} ; t_{0}\right]=\sum_{J} \widetilde{\mathfrak{N}}_{I}^{J} \mathcal{J}_{J}\left[z_{f}\right]
$$

But since $\widetilde{\mathcal{J}}_{I}\left[\vec{z}_{i} ; t_{0}\right]$ parameterizes flows on the interval $\left(-\infty, t_{0}\right]$ that start at $I$, a flow from $\widetilde{\mathcal{J}}_{I}\left[\vec{z}_{i} ; t_{0}\right]$ to $J$ on the interval $\left[t_{0}, \infty\right)$ is equivalent to a flow from $I$ to $J$ defined on the whole real line. So the $\widetilde{\mathfrak{N}}_{I}^{J}$ are the same as the desired invariants $\mathfrak{N}_{I}^{J}$ :

$$
\widetilde{\mathcal{J}}_{I}\left[\vec{z}_{i} ; t_{0}\right]=\sum_{J} \mathfrak{N}_{I}^{J} \mathcal{J}_{J}\left[z_{f}\right]
$$

The relative homology is defined over $\mathbb{Z}$, and an integral relative homology class such as $\widetilde{\mathcal{J}}_{I}$ has no continuous deformations. So clearly, as long as the continuous deformation from $\mathcal{J}_{I}\left[\vec{z}_{i}\right]$ to $\widetilde{\mathcal{J}}_{I}\left[\vec{z} ; t_{0}\right]$ induced by the flow equations (4.14) lives at any given time in $H_{m}\left(\mathcal{U}, \mathcal{U}_{<}\right) \vec{z}(t)$, it coincides with the natural transport along $\mathcal{P}$ by the Gauss-Manin connection. In this case, (4.21) is equivalent to the desired result $\mathfrak{N}_{I}^{J}=\mathfrak{n}_{I}^{J}$.

To show that $\widetilde{\mathcal{J}}_{I}\left[\vec{z} ; t_{0}\right]$ lies in $H_{m}\left(\mathcal{U}, \mathcal{U}_{<}\right)_{\vec{z}\left(t_{0}\right)}$ for any $t_{0}$, we are supposed to prove that $\operatorname{Re} \mathcal{W}\left(w, \vec{z}\left(t_{0}\right)\right)$ goes to $-\infty$ at infinity along $\widetilde{\mathcal{J}}_{I}\left[t_{0}\right]$. Indeed, if a sequence of forced gradient flows on the semi-infinite interval $\left(-\infty, t_{0}\right]$ goes to infinity, it does so by diverging for $t \rightarrow t_{0}$, in which case $\operatorname{Re}\left(\mathcal{W}\left(w, z\left(t_{0}\right)\right)\right.$ (whose gradient drives the flow for $t \rightarrow t_{0}$ ) must go to $-\infty$.

An alternative approach to (4.16) is the following. Suppose that $\vec{z}_{f}=\vec{z}_{i}$ and $\mathcal{P}$ is the trivial path between them. Then $\mathfrak{N}_{I}^{J}=\mathfrak{n}_{I}^{J}=\delta_{I}^{J}$. As we vary $\vec{z}_{f}$, both $\mathfrak{N}_{I}^{J}$ and $\mathfrak{n}_{I}^{J}$ may jump in and only in crossing Stokes walls; they jump in exactly the same way, so they remain equal. We have already described the jumping of $\mathfrak{n}_{I}^{J}$. The jumping of $\mathfrak{N}_{I}^{J}$ occurs because in crossing a Stokes wall, a time-dependent solution may disappear to infinity, as follows. Suppose that, for $\vec{z}_{f}$ on some Stokes wall, there is a jump in $\mathfrak{n}_{I}^{J}$, resulting from an 
ordinary gradient flow from some critical point $J^{\prime}$ of $\mathcal{W}\left(w, \vec{z}_{f}\right)$ to $J$. Such a flow produces a jump

$$
\mathfrak{n}_{I}^{J} \rightarrow \mathfrak{n}_{I}^{J} \pm \mathfrak{n}_{I}^{J^{\prime}}
$$

where the sign depends on the direction in which one crosses the Stokes wall. To see a corresponding jump in $\mathfrak{N}_{I}^{J}$, one looks for forced gradient trajectories from $I$ to $J$ that consist of a forced gradient trajectory from $I$ to $J^{\prime}$ followed, at some time very far in the future, by the same ordinary gradient trajectory from $J^{\prime}$ to $J$ that causes the jump of $\mathfrak{n}_{I}^{J}$. A two-step forced trajectory of this kind exists if $\vec{z}_{f}$ is near the Stokes wall and on the proper side of it; the time at which the second step of the flow occurs diverges as $\vec{z}_{f}$ crosses the Stokes wall. This leads to the disappearance of the two-step solution and a jump of $\mathfrak{N}_{I}^{J}$ that just matches the jump of $\mathfrak{n}_{I}^{J}$.

\section{From braiding of thimbles to free-field integrals}

According to the reasoning in Section 4, to understand the braid group representations associated to the Jones polynomial, we are supposed to compute a natural monodromy action on the middle-dimensional relative homology of an infinite-dimensional space $\mathcal{U}$ of connections on a threemanifold $M_{3}=C \times \mathbb{R}_{+}$. This may sound hopelessly abstract. We will now show how it can be turned into something concrete and calculable.

\subsection{From thimbles to integrals}

A convenient way to describe the evolution of states in $\mathcal{H}$, including the $q$-grading, is to view the homology cycles as integration cycles. Instead of looking at the evolution of the homology cycles, it is equivalent to look at the evolution of the integrals. Of course, we need an integrand which can be integrated on the thimbles $\mathcal{J}_{I}$, which are not compact. A function that fills the bill is $\mathrm{e}^{\mathcal{W} / \varepsilon}$, where $\varepsilon$ is chosen in a suitable half-plane. Since

$$
h=\operatorname{Re}\left(\mathrm{e}^{\mathrm{i} \alpha} \mathcal{W}\right)
$$

goes to minus infinity along a thimble, the condition we want is $\operatorname{Re}\left(\mathrm{e}^{\mathrm{i} \alpha} \varepsilon\right)>$ 0 . This will ensure the convergence of the integrals

$$
\mathcal{I}_{\Gamma}=\oint_{\Gamma} \mathrm{e}^{\mathcal{W}(w) / \varepsilon} d \Omega
$$


where $d \Omega$ is a holomorphic volume form on $\mathcal{U}$ (which will be kept fixed in what follows) and the integration cycle $\Gamma$ is a thimble, or more generally any cycle in the relative homology $H\left(\mathcal{U}, \mathcal{U}_{<}\right)$.

The thimbles are particularly nice integration cycles, because the $\varepsilon \rightarrow 0$ limit of the integral over a thimble is very simple. On a thimble $\mathcal{J}_{I}$ defined by gradient flow from a critical point $I$, the function $h$ has a unique maximum, namely the critical point. So for $\varepsilon \rightarrow 0$, the integral over a thimble is

$$
\mathcal{I}_{I}:=\oint_{\mathcal{J}_{I}} \mathrm{e}^{\mathcal{W}(w) / \varepsilon} d \Omega \sim \exp \left(\mathcal{W}_{I} / \varepsilon\right)\left(\varepsilon^{-\operatorname{dim} \mathcal{J}_{I} / 2} c_{0}+\cdots\right)
$$

where $\mathcal{W}_{I}$ is the value of $\mathcal{W}$ at the critical point $I$.

This formula is valid throughout the half-plane $\operatorname{Re}\left(\mathrm{e}^{\mathrm{i} \alpha} \varepsilon\right)>0$, but actually as long as $\alpha$ is not on a Stokes wall, this asymptotics holds in a slightly larger sector in the complex plane. ${ }^{10}$ This property uniquely characterized the basis of integrals $\mathcal{I}_{I}$ among all the possible $I_{\Gamma}$ : if we were to take a linear combination of several $\mathcal{I}_{I}$, the asymptotics would fail at some ray in the extended half-plane where two critical points exchange dominance. This characterization is familiar in Stokes theory, and motivated the terminology "Stokes walls".

As we vary the parameters of $\mathcal{W}$, the homology $H\left(\mathcal{U}, \mathcal{U}_{<}\right)$will vary continuously. If we vary the integration cycle $\Gamma$ continuously, the integral will vary holomorphically in the parameters of $\mathcal{W}$. The monodromy of the cycles $\Gamma$ is the same as the monodromy of the integrals $\mathcal{I}_{\Gamma}$.

\subsection{Two Chern-Simons theories}

In our present context, the thimble integral (5.2) is a Chern-Simons path integral on the three manifold $M_{3}=C \times \mathbb{R}_{+}$, albeit on an unusual integration cycle. Such an integral can be concretely expressed in terms of $\mathcal{N}=4$ gauge theory on $M_{3} \times \widetilde{\mathbb{R}}_{+}$. (In this section only, we write $\widetilde{\mathbb{R}}$ or $\widetilde{\mathbb{R}}_{+}$for the

\footnotetext{
${ }^{10}$ For the stated asymptotics to break down, the first step is to cross a Stokes wall, so that the thimble we started with evolves into a linear combination of thimbles with at least two terms. Initially, the asymptotics (5.3) remain valid, as any extra thimbles that appear at the Stokes wall initially make exponentially small contributions. If one varies $\alpha$ further, one of the extra thimbles may eventually become dominant. The combined process always involves varying $\alpha$ by an angle strictly greater than $\pi / 2$ from its initial value. To show this, one just compares the values of $\mathcal{W}$ at the two critical points; these values have equal imaginary parts at the Stokes wall, and equal real parts when the two critical points exchange dominance.
} 
$x^{1}$ direction to distinguish it from the $y$-direction $\mathbb{R}_{+}$.) This statement was one of the main conclusions of $[40,41]$. In our case, since $M_{3}=C \times \mathbb{R}_{+}$, the four manifold is $M_{4}=C \times \mathbb{R}_{+} \times \widetilde{\mathbb{R}}_{+}$.

This is the second Chern-Simons theory to appear in this paper, at least implicitly. Our whole analysis concerns the calculation of the Jones polynomial, in a gauge theory setup which is $S$-dual to a setup which computes the Jones polynomial by Chern-Simons theory on $W=C \times \widetilde{\mathbb{R}}$. In that "original" Chern-Simons theory, the knot is a Wilson loop, the gauge group is $G^{\vee}$, and the coupling parameter is $\boldsymbol{k}^{\vee}$. The Jones polynomial is a Laurent polynomial in

$$
q=\exp \left(2 \pi \mathrm{i} /\left(\boldsymbol{k}^{\vee}+2\right)\right),
$$

where 2 is the dual Coxeter number of $S U(2)$.

As explained in [14], Chern-Simons theory on a three-manifold $W$ can be computed via topologically twisted $\mathcal{N}=4$ super Yang-Mills theory on $W \times \mathbb{R}_{+}$if one relates $\boldsymbol{k}^{\vee}$ to the twisting parameter $\Psi^{\vee}$ of the $\mathcal{N}=4$ theory by

$$
\Psi^{\vee}= \pm\left(\boldsymbol{k}^{\vee}+2\right)
$$

In this description one uses a D3-NS5 boundary condition at the origin in $\mathbb{R}_{+}$. The sign \pm depends on the relative choice of orientation between $M_{4}$ and $W$.

One can also apply $S$-duality, converting the gauge group from $G^{\vee}=$ $S U(2)$ to $G=S O(3)$ and converting the D3-NS5 boundary condition to a D3-D5 boundary condition; this boundary condition involves a Nahm pole, as explained in [19]. In this new description, which has been the starting point of the present paper, the twisting parameter is $\Psi=-1 / \Psi^{\vee}$. Because this dual description is difficult, we have tried to simplify it, as first explained in Section 1.2, by "stretching" $W$ in one direction. Thus, we approximated $W$ by $\widetilde{\mathbb{R}} \times C$, and looked for solutions of the BPS equations on $W \times \mathbb{R}_{+}$that are "pulled back" from $M_{3}=C \times \mathbb{R}_{+}$. If this dual description is formulated as supersymmetric quantum mechanics, with $\mathbb{R}$ as the time direction and the field variables being gauge fields on $M_{3}=C \times \widetilde{\mathbb{R}}_{+}$, then the superpotential is $\Psi\left(\right.$ not $\left.\Psi^{\vee}\right)$ times the Chern-Simons function.

We then relate the braiding of solutions on $M_{3}$ to the braiding of thimbles for Chern-Simons theory on $M_{3}$, which we express by $\mathcal{N}=4$ theory on $M_{3} \times \widetilde{\mathbb{R}}_{+}$. In this description, we impose D3-NS5 boundary conditions at the origin of $\widetilde{\mathbb{R}}_{+}$(and D3-D5 at the origin of $\mathbb{R}_{+}$). The level $k$ of this 
Chern-Simons description is related to the twisting parameter of the $\mathcal{N}=4$ theory by

$$
\Psi=\mp(\boldsymbol{k}+2) .
$$

The two Chern-Simons descriptions are related by $S$-duality, together with the exchange of the roles of $\mathbb{R}_{+}$and $\widetilde{\mathbb{R}}_{+}$. The opposite sign in (5.5) and (5.6) is due to the fact that exchanging $\mathbb{R}_{+}$and $\widetilde{\mathbb{R}}_{+}$reverses the orientation of $M_{4}$.

Combining this with $\Psi=-1 / \Psi^{\vee}$ and $\Psi^{\vee}=\boldsymbol{k}^{\vee}+2$, we find that the relation between the level $\boldsymbol{k}^{\vee}$ in the Chern-Simons description that is related to the Jones polynomial in the traditional way and the level $\boldsymbol{k}$ in the ChernSimons description that relates the Jones polynomial to Nahm poles and opers is

$$
\boldsymbol{k}+2=1 /\left(\boldsymbol{k}^{\vee}+2\right)
$$

This four-dimensional setup, with the $\mathcal{N}=4$ theory on a manifold $M_{4}=$ $C \times \mathbb{R}_{+} \times \widetilde{\mathbb{R}}_{+}$with a "corner", is rather interesting, and we believe it deserves to be explored further. We will not do so in this paper.

\subsection{From Chern-Simons to conformal blocks}

For this paper, more useful than the relation of Chern-Simons theory on $M_{3}=C \times \mathbb{R}_{+}$to four dimensions is its relation to conformal blocks on $C$. The most familiar version of this statement [44] is that a path integral with $\mathcal{A}_{\bar{z}}$ fixed and $\mathcal{A}_{z}$ varying gives a Wess-Zumino-Witten (WZW) conformal block (that is, a conformal block in two-dimensional current algebra, with the symmetry group $G$ being the same as the gauge group of the ChernSimons theory). Local variations of the fixed value of $\mathcal{A}_{\bar{z}}$ insert a holomorphic current $J(z)$ in the conformal block:

$$
\delta \log \mathcal{Z}=\int \operatorname{Tr}\left\langle J_{z}\right\rangle \delta A_{\bar{z}}
$$

This statement has an analog [45] that leads to Virasoro conformal blocks in the case of $S U(2)$ or $S O(3)$ gauge theory, or to more general $W$-algebra conformal blocks in the case of gauge groups of higher rank [46, 47]. This analog involves a different boundary condition in which, at the price of breaking some gauge symmetry at the boundary, one fixes some parts of 
$\mathcal{A}_{z}$, and some parts of $A_{\bar{z}}$. For gauge group $S U(2)$, the boundary condition which leads to Virasoro conformal blocks is simply stated:

$$
\mathcal{A}_{z}=\left(\begin{array}{cc}
* & 1 \\
* & *
\end{array}\right) \quad \mathcal{A}_{\bar{z}}=\left(\begin{array}{cc}
\times & 0 \\
* & \times
\end{array}\right)
$$

Here we denote as $*$ the elements which are free to vary, and as $\times$ elements which are fixed.

The connection just described is an oper! (Since $\mathcal{A}_{\bar{z}}$ is lower-triangular, a bundle with this connection has a holomorphic sub-bundle $L$ whose sections are of the form $\left(\begin{array}{l}0 \\ *\end{array}\right)$, and because the upper right matrix element of $\mathcal{A}_{z}$ nowhere vanishes, this sub-bundle is nowhere preserved by $\mathcal{D}_{z}=$ $\partial_{z}+\left[\mathcal{A}_{z}, \cdot\right]$.) So the complex boundary condition (5.9) is the one that is induced by the Nahm pole boundary condition studied in Section 3. To explain the relation to Virasoro conformal blocks, we note that the Nahm pole boundary condition depends on a choice of complex structure on $C$. Once a complex structure is picked with a local complex coordinate $z$, nearby complex structures can be described by a Beltrami differential $\mu_{z}^{z}$. The relation is that in the new complex structure, the holomorphic fields on the space of complex connections are not $\mathcal{A}_{\bar{z}}$ but $\mathcal{A}_{\bar{z}}-\mu_{\bar{z}}^{z} \mathcal{A}_{z}$. Making this deformation is equivalent to replacing the " 0 " in the boundary condition for $\mathcal{A}_{\bar{z}}$ in (5.9) with $\mu_{\bar{z}}^{z}$ (so that now $\mathcal{A}_{\bar{z}}-\mu_{\bar{z}}^{z} \mathcal{A}_{z}$ is lower triangular). Hence a local variation of the boundary condition associated to a change in complex structure inserts a holomorphic stress tensor

$$
\delta \log \mathcal{Z}=\int\left\langle T_{z z}\right\rangle \delta \mu_{\bar{z}}^{z}
$$

and this leads to the relation between Chern-Simons theory with the oper boundary condition and Virasoro conformal blocks.

In the semiclassical limit, the operator $T_{z z}$ reduces to the classical stress tensor $t(z)[\mathcal{A}]$ of the oper. More precisely, the identification of parameters from $[45]$ is that if we define

$$
-b^{-2}=\boldsymbol{k}+2,
$$

with $\boldsymbol{k}$ the Chern-Simons level, then the stress-tensor has central charge $c=1+6\left(b+b^{-1}\right)^{2}$ and in the semiclassical limit, $b^{2} T(z) \rightarrow t(z)[\mathcal{A}]$.

If we combine (5.11) with (5.7) and (5.4), we find the relationship between the variable $q$ usually used in describing the Jones polynomial and the 
parameter $b$ used in describing Virasoro conformal blocks:

$$
q=\exp \left(-2 \pi \mathrm{i} / b^{2}\right)
$$

We have here assumed that $G$ is $S U(2)$ or $S O(3)$. For general gauge group $G$, both Nahm poles and $W$-algebras are labeled by an $\mathfrak{s u}(2)$ embedding in the Lie algebra $\mathfrak{g}$ of $G$. Inspection confirms that the boundary conditions used to define a general $W$-algebra conformal block are induced by the corresponding Nahm pole.

\subsubsection{Analog for Liouville}

Though we will not need this fact in the present paper, we should remark that the relation between Virasoro conformal blocks and Chern-Simons theory has a simple extension to a relation beween Liouville theory and ChernSimons theory. To do Liouville theory on a Riemann surface $C$, one considers Chern-Simons theory on $C \times I$ where $I$ is a unit interval. At one end of $I$, one imposes the Nahm pole boundary condition and at the other end, one imposes a variant of the Nahm pole boundary condition with $z$ and $\bar{z}$ exchanged. Liouville partition functions and correlation functions are built by combining holomorphic and anti-holomorphic Virasoro conformal blocks, which arise naturally from Chern-Simons on $C \times I$ with boundary conditions just stated. (For the case of a compact symmetry group, it is already known that Chern-Simons on $C \times I$ reproduces the WZW model on $C$.) By slightly extending arguments that we present presently, light degenerate fields and generic primary fields of Liouville theory, inserted at a point $p \in C$, correspond to Wilson operators or monodromy defects on $p \times I$.

In the classical limit, the correspondence between Chern-Simons and Liouville theory means the following. A classical solution of Chern-Simons theory on $C \times I$ with boundary conditions as above is a flat bundle on $C$ whose holomorphic and antiholomorphic structures both obey the oper condition. Indeed, a classical solution of Liouville theory corresponds to a metric on $C$ of constant negative curvature. If $\omega$ and $e$ are the vierbein and spin connection of this metric, then we can define a corresponding $S L(2, \mathbb{R})$ flat connection $\mathcal{A}=\omega \boldsymbol{t}_{3}+e_{z} \boldsymbol{t}_{+}+e_{\bar{z}} \boldsymbol{t}_{-}$. With a standard representation of the $\boldsymbol{t}_{i}, \mathcal{A}_{z}$ is upper triangular with an upper right matrix element that is everywhere nonzero, and $\mathcal{A}_{\bar{z}}$ is lower triangular with a lower left matrix element that is everywhere nonzero. So both the holomorphic and antiholomorphic structures defined by this flat connection satisfy oper conditions, as expected in the Chern-Simons description. (The antiholomorphic oper structure is defined with the roles of "upper triangular" and "lower triangular" matrices 
reversed.) So this gives the mapping between the two theories at the classical level.

\subsection{Wilson line operators}

To further understand the mapping from three-dimensional Chern-Simons theory with oper boundary conditions to Virasoro conformal blocks in two dimensions, we will explore the interpretation of Wilson line operators. First, let us recall what happens if one uses standard boundary conditions that relate Chern-Simons theory to current algebra. In this case, a Wilson line operator ending on the boundary of a three-manifold $M_{3}$ represents insertion of a conformal primary field at that boundary point in the WZW conformal block. If the Wilson line operator transforms in a finitedimensional representation $R$ of $G$, then the corresponding conformal primary field transforms in the same representation. This is consistent with the fact that a Wilson line operator ending on the boundary is not gaugeinvariant, but transforms in the representation $R$, just like the corresponding primary field of the WZW model.

What is the analogous interpretation of a Wilson line operator that ends on a boundary at which one imposes Nahm pole boundary conditions? The Nahm pole breaks the gauge symmetry at the boundary, so we have to pick a component of the Wilson line operator. As we discussed in Section 3.2 , a generic vector diverges when parallel transported to the boundary. Given a Wilson line operator ending at $y=0$, the most easily defined gauge invariant information is the coefficient of the most negative power of $y$. This is extracted simply by contracting with an appropriate power of the small section $s$. Actually, we will find useful a rescaled version of $s$, namely $\widehat{s}=K(z)^{-1 / 2} s(z)$, which satisfies

$$
\left(\mathcal{D}_{z}^{2}+t(z)\right) \widehat{s}=0 \quad \mathcal{D}_{\bar{z}} \widehat{s}=0 \quad \mathcal{D}_{y} \widehat{s}=0,
$$

and has definite conformal dimension $-1 / 2$. In the gauge $(3.46), \widehat{s}=\left(\begin{array}{l}0 \\ 1\end{array}\right)$.

In the two-dimensional representation of $S U(2)$, we would consider an operator

$$
P \exp \left(-\int_{\gamma} \mathcal{A}\right) \widehat{s}(z)
$$

where $\gamma$ is a path ending on the boundary at $y=0$. In the classical limit, under conformal transformations of the boundary, this has the same conformal 
dimension as $\widehat{s}$, i.e., $-1 / 2$. For a spin $k / 2$ representation, one must contract with $k$ powers of $\widehat{s}$ and the classical limit of the dimension is $-k / 2$.

We want to argue now that a Chern-Simons path integral with a spin $k / 2$ Wilson operator ending on the boundary gives a Virasoro conformal block with the insertion of a "light" degenerate field of Liouville momentum $-b k / 2$. Such a field has the correct classical dimension $-k / 2$ in the $b \rightarrow 0$ limit, and furthermore the exact formula for its quantum dimension

$$
-\frac{k}{2} b\left(b+\frac{1}{b}+\frac{k}{2} b\right)=-\frac{k}{2}-\frac{k(k+2)}{4} b^{2}=-\frac{k}{2}+\frac{k(k+2)}{4(k+2)}
$$

is the sum of the classical dimension of $\widetilde{s}^{k}$ and the dimension of a spin $k / 2$ operator in a WZW model (of level $\boldsymbol{k}+2=-1 / b^{2}$ as in (5.11)). Furthermore, the operator (5.14) satisfies classically the correct differential equation: the $k=1$ operator is annihilated by $\partial_{z}^{2}+t(z)$, etc.

Part of what makes possible the correspondence between spin $k / 2$ Wilson lines and degenerate conformal fields possible is that the degenerate fields satisfy fusion rules which coincide with the fusion rules of spin $k / 2$ operators in the WZW model. This last fact is part of the input in the statement that the braid group representations associated to the Jones polynomial can be computed by the braiding of either primary fields of the WZW model or degenerate conformal fields of the Virasoro algebra.

\subsection{Singular monopoles and "heavy" degenerate fields}

Next, we would like to identify in the Chern-Simons description of Virasoro conformal blocks the "heavy" degenerate fields of Liouville momentum $-k / 2 b$. These are the degenerate fields whose conformal dimension diverges for $b \rightarrow 0$. We claim that they correspond to the insertion of singular monopoles at the boundary.

The main insight of Section 3.3 was that at a Nahm boundary with singular monopoles (and generic $\zeta$ ), the connection is an oper with singularities of trivial monodromy. We observed that the classical stress tensor of such an oper has poles that agree with the semiclassical limit of the quantum stress tensor in the presence of a heavy degenerate field. Moreover, the trivial monodromy condition holds quantum-mechanically as well: a light degenerate field has no monodromy around a heavy degenerate field, as they fuse in a unique channel. 
In the Chern-Simons setup, the classical trivial monodromy condition follows naturally from the fact that the singular monopole does not extend in the bulk. This was part of our derivation in Section 3.3. Quantum mechanically, we need to consider the behavior when a light degenerate field represented in three dimensions by an expression such as $P \exp \left(-\int_{\gamma} \mathcal{A}\right) \widehat{s}$ - approaches the singular monopole. The Wilson loop itself is topological, and the small section $\widehat{s}$ has trivial monodromy around the singular monopole.

\subsection{Putting the pieces together}

We can now finally establish a link between the solutions of the fourdimensional BPS equations (1.1) that we started with and the braid group representations associated to the Jones polynomial.

The time-independent solutions of the BPS equations correspond to opers with trivial monodromy. We have identified the braiding of the corresponding quantum states with the braiding of complex integration cycles for Chern-Simons theory, and then with the braiding of degenerate Virasoro conformal blocks. These are known $[34,35]$ to be the braid group representations associated to Jones polynomials. So we have arrived at our goal, although in a form that may sound a little abstract.

We can put this result in a perfectly concrete form using the free-field representation of the conformal blocks. Opers with trivial monodromy are also associated to critical points of the Yang-Yang function for the Bethe equations; this is the logarithm of the integrand in the free-field realization of conformal blocks. We can derive a degenerate Virasoro conformal block either from an infinite-dimensional thimble associated to an oper with trivial monodromy, or from a finite-dimensional thimble associated to a critical point of the Yang-Yang function. Either way, we get a conformal block with definite and uniform semiclassical limit in a sector of angular width greater than $\pi$ in the $b^{2}$ plane. As those are unique, the two bases of conformal blocks must coincide.

Hence the braiding representations associated to the four-dimensional gauge theory coincide with the braiding representations of integration cycles in the space of Bethe parameters $w_{i}$. This is not as surprising as it may seem if we turn on a symmetry breaking parameter: then we have interpreted the $w_{i}$ as positions of bulk BPS monopoles, and our claim possibly amounts to the statement that the four-dimensional nonabelian gauge theory on the Coulomb branch reduces to a theory of massive monopoles and abelian gauge fields. We will develop this point of view further in Section 7. 


\section{Braiding representations of integration cycles}

\subsection{Overview}

A highlight of what we have learned so far is the existence of a natural map from the braid group representations derived from the four-dimensional gauge theory equations (1.1) to the braid group representations associated to correlation functions of Virasoro degenerate fields. Those braid group representations can be effectively studied using the free-field representation, which we reviewed in Section 3.7. Making this explicit will be our goal here.

We consider a degenerate correlation function $\left\langle\prod_{a=1}^{d} V_{-k_{a} / 2 b}\left(z_{a}\right)\right\rangle$. We assume that the $z_{a}$ are distinct points in $\mathbb{C}$. To represent conformal blocks, we introduce $q$ variables $w_{i} \in \mathbb{C}$, which we assume to be distinct from each other and from the $z_{a}$. The allowed values of $q$ have been analyzed in Section 3. Moreover, we consider the $w_{i}$ to be indistinguishable, in the sense that configurations that differ by permuting them are equivalent. We write $\mathcal{M}$ for the space of such distinct and indistinguishable variables $w_{i} \in$ $\mathbb{C} \backslash\left\{z_{1}, \ldots, z_{d}\right\}$. We also write $\widehat{\mathcal{M}}$ for the smallest cover of $\mathcal{M}$ on which the Yang-Yang function $\mathcal{W}$ of equation (3.52) is single-valued.

In the free-field representation, degenerate conformal blocks are written in the form

$$
\int_{\Gamma} \exp \left(\mathcal{W} / b^{2}\right) d w_{1} \ldots d w_{q}
$$

$\Gamma$ is a middle-dimensional cycle in $\widehat{\mathcal{M}}$, chosen so that the integral converges. ${ }^{11}$

Morse theory offers a systematic way to produce all such integration cycles: a basis of integration cycles is given by the thimbles associated to critical points of $\mathcal{W}$. Cycles of this kind are never compact; they have noncompact ends on which the Morse function $h=\operatorname{Re} \mathcal{W}$ goes to $-\infty$. In our

\footnotetext{
${ }^{11}$ As explained in [34], the cycle $\Gamma$ should actually be odd under the exchange of any pair of $w$ 's, to compensate for the sign change of the differential form $d w_{1} \wedge \cdots \wedge d w_{q}$ under permutations. This means that the appropriate relative homology is actually the part that is antisymmetric under permutations of the $w$ 's. To be concrete, suppose that there are two $w$ 's and we find a solution of the Bethe equations at which the $w$ 's equal $\alpha$ and $\beta$ up to permutation. Then we can define a cycle $\mathcal{C}^{\prime}$ associated to the critical point $w_{1}=\alpha, w_{2}=\beta$, and a cycle $\mathcal{C}^{\prime \prime}$ associated to the critical point $w_{1}=\beta, w_{2}=\alpha$. The difference $\mathcal{C}^{\prime}-\mathcal{C}^{\prime \prime}$ is an element of the antisymmetric part of the homology. In practice, we can omit to explicitly form such differences and also ignore minus signs arising from permutations of the factors in $d w_{1} \wedge \cdots \wedge d w_{n}$.
} 
problem, this happens when one of the $w_{i}$ either approaches one of the $z_{a}$ or, in the presence of symmetry breaking, goes to infinity in the correct direction. In simple situations, instead of using Morse theory, one can describe integration cycles by hand. In constructing a cycle $\Gamma$ by hand, one has to make sure that the Morse function really goes to $-\infty$ at infinity along $\Gamma$. For example, this will fail if too many $w_{i}$ approach simultaneously the same $z_{a}$.

With symmetry breaking, some of the important integration cycles have ends at $w=\infty$ and the use of noncompact integration cycles is unavoidable. However, in the absence of symmetry breaking, the noncompact integration cycles produced by Morse theory have their ends at $w_{i} \rightarrow z_{a}$, for various $i$ and $a$, and are equivalent in the appropriate twisted relative homology to compact cycles in which the $w_{i}$ wrap around the $z_{a}$ in a suitable fashion. (For an example, see figure 7 below.) In the extensive literature on integration cycles in free-field realizations of conformal blocks $[32,33]$ and their application to the Jones polynomial $[34,35]$, compact integration cycles are often used. Symmetry breaking, or in other words the introduction of an irregular singularity at infinity, has not been considered in this context, as far as we know.

The use of Morse theory has advantages and disadvantages. The main disadvantage is that the thimbles do not correspond to a standard BPZ basis of conformal blocks defined by fusing the degenerate fields in specific channels. The main advantage is that in the basis of thimbles, the braid group is manifestly represented by matrices whose entries are Laurent polynomials in $q$ with integer coefficients. This property, which was explained in Section 4.1.4, is important vis-a-vis the Jones polynomial and Khovanov homology.

In what follows, we will first analyze a few important examples with a small number of degenerate insertions $z_{a}$ and Bethe roots $w_{i}$, with or without symmetry breaking. Then in Section 6.5, we analyze the general case in the presence of symmetry breaking. From that analysis, we get the experience we need to deduce a general description of the Jones polynomial - not just the associated braid group representations. This is presented in Section 6.7.

\subsection{1 $S U(2)$ versus $S O(3)$}

We pause for a technical remark concerning the assertion that the entries of the braiding matrices are Laurent polynomials in $q$.

If the gauge group is $G=S U(2)$, meaning that the dual group is $G^{\vee}=$ $S O(3)$ and the charges $k_{a}$ of the singular monopoles are all even, then the Yang-Yang function $\mathcal{W}$ as defined in (3.52) is well-defined mod $2 \pi i$. Hence a change of branch of $\mathcal{W}$ multiplies $\exp \left(\mathcal{W} / b^{2}\right)$ by an integer power 
of $q=\exp \left(-2 \pi \mathrm{i} / b^{2}\right)$, and the braiding matrices are Laurent polynomials in $q$ with integer coefficients.

If instead $G=S O(3), G^{\vee}=S U(2)$, then some of the $k_{a}$ may be odd. (Indeed, we will do our detailed computations for the case that all $k_{a}$ are 1.) Then $\mathcal{W}$ is well-defined $\bmod 2 \pi \mathrm{i}$ if the $w_{i}$ are varied for fixed positions $z_{a}$ of the knots, but is only well-defined $\bmod 2 \pi \mathrm{i} / 4$ when the $z_{a}$ are varied. Consequently, for $G=S O(3)$, the braid matrices will actually be Laurent polynomials in $q^{1 / 4}$.

Of course, we could eliminate this by writing the formulae in terms of $\widetilde{q}=q^{1 / 4}$, but we prefer not to do so since $q$ as we define it is the natural instanton counting parameter in four dimensions. The underlying reason for the difference between $G=S U(2)$ and $G=S O(3)$ is that the Chern-Simons function, normalized as we have done in (3.6), is gauge-invariant mod $2 \pi \mathrm{i}$ in $S U(2)$ gauge theory, but gauge-invariant $\bmod 2 \pi \mathrm{i} / 4$ in $S O(3)$ gauge theory. The last statement holds on any sufficiently rich three-manifold $M_{3}$, or on any $M_{3}$ if singular monopoles of odd charge are present. In the latter case, one considers only gauge transformations that are trivial at the position of the singular monopole.

\subsection{A single critical point}

We begin with the two examples in which the Yang-Yang function has only a single critical point.

\subsubsection{Braiding of two primaries with minimal charge}

The first example arises in the absence of symmetry breaking, with two $z_{a}$ of charge $k=1$ and one $w$. An obvious integration cycle $\mathcal{C}_{12}$ is a segment joining the two $z_{a}$. Now, let us compare it with the thimble. The Bethe equation

$$
\frac{1}{w-z_{1}}+\frac{1}{w-z_{2}}=0
$$

has a unique solution $w=\frac{1}{2}\left(z_{1}+z_{2}\right)$. The thimble flows down from $w$ to the $z_{i}$ along a straight line, and coincides with the obvious cycle $\mathcal{C}_{12}$. The critical value of the Yang-Yang function

$$
\mathcal{W}=-\frac{1}{2} \log \left(z_{1}-z_{2}\right)+\log \left(w-z_{1}\right)+\log \left(w-z_{2}\right)=\frac{3}{2} \log \left(z_{1}-z_{2}\right)+\text { const. }
$$


is such that $\exp \left(\mathcal{W} / b^{2}\right)$ agrees with the OPE coefficient of the two degenerate fields in the identity channel $V_{-1 / 2 b}\left(z_{1}\right) V_{-1 / 2 b}\left(z_{2}\right) \sim\left(z_{1}-z_{2}\right)^{\frac{3}{2 b^{2}}}$. (Recall that for small $b$, the dimension of $V_{-1 / 2 b}$ is $-3 / 4 b^{2}+\mathcal{O}(1)$.) This is expected, because with two $z_{a}$ and one $w$ the oper has no singularity at infinity, in view of the discussion of equation (3.22), and hence describes the fusing of two fields to the identity.

In order to fuse the two degenerate fields in a channel of momentum $-b$ we would consider something even simpler: a case without any w's. In this channel, the conformal block is simply the free-field correlation function $\left\langle V_{-1 / 2 b}\left(z_{1}\right) V_{-1 / 2 b}\left(z_{2}\right)\right\rangle$ with no integral at all. With no $w$ 's, the discussion of (3.22) shows that the oper does have a singularity at infinity - corresponding to fusion of the two $k=1$ degenerate primary fields to a $k=2$ degenerate primary field.

To be precise, in defining an integration cycle such as $\mathcal{C}_{12}$, we should specify a choice of branch of $\mathcal{W}$. (Differently put, the cycle is supposed to be defined in the covering space $\widehat{\mathcal{M}}$.) If we braid $z_{1}$ around $z_{2}, \mathcal{C}_{12}$ evolves continuously, but we may end up with a different branch of $\mathcal{W}$. As the charges at $z_{1}$ and $z_{2}$ are identical, a basic braiding move is to exchange the position of $z_{1}$ and $z_{2}$, and then relabel them. In the absence of $w$, we would only have the factor $\left(z_{1}-z_{2}\right)^{-\frac{1}{2 b^{2}}}$ coming from the part of $\mathcal{W}$ which only depends on $z_{a}$, so a braiding which exchanges $z_{1}$ and $z_{2}$ counterclockwise would give a factor of $\mathrm{e}^{-\frac{\mathrm{i} \pi}{2 b^{2}}}=q^{\frac{1}{4}}$. In the presence of one $w$, we can get the result by following the value of $\mathcal{W}$ at the midpoint of $\mathcal{C}_{12}$ :

$$
\mathcal{C}_{12} \rightarrow-q^{-\frac{3}{4}} \mathcal{C}_{12}
$$

The minus sign follows from the change in orientation of $\mathcal{C}_{12}$.

It is interesting to compare the thimble with closed contours which are commonly used in order to describe BPZ conformal blocks, as depicted in figure 7. As illustrated in the picture, these contours are equivalent in homology to the thimble times a Laurent polynomial in $q$. From the point of view of the Stokes matrices, they are not as elementary as the thimble.

\subsubsection{One primary field with symmetry breaking}

The other basic example with one critical point occurs in the presence of symmetry breaking with a single degenerate field of $k=1$, and a single Bethe root. The Bethe equation reads

$$
\frac{1}{w-z}=c \quad c=-\frac{2 a}{\zeta} .
$$



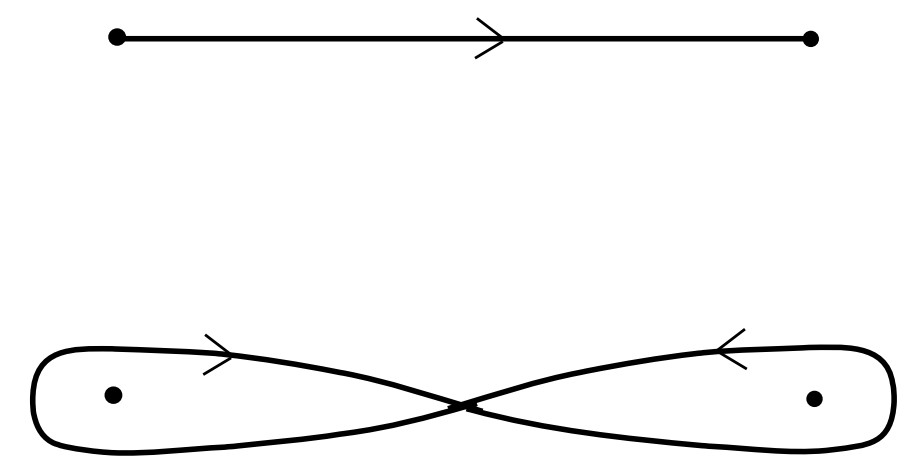

Figure 7: The thimble $\mathcal{C}_{12}$ (top) compared to a closed integration contour (bottom), which is equivalent to $\left(1-q^{-1}\right) \mathcal{C}_{12}$.

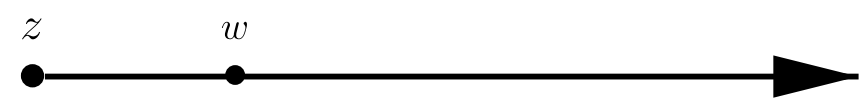

Figure 8: This ray parallel to the real axis is the Lefschetz thimble for the case of one primary field of minimal charge with symmetry breaking.

So $w=z+\frac{1}{c}$. For convenience, we will take the constant $c$ to be real and positive. The thimble again coincides (figure 8 ) with the most natural integration cycle $\mathcal{C}$, along a ray starting at $z$ and parallel to the positive real axis, passing through $w$. This example illustrates that an integration cycle in the presence of symmetry breaking may end at infinity. The cycle that we have just described cannot be replaced with an equivalent compact cycle.

\subsection{Two critical points}

Next we can consider examples with only one Bethe root $w$, but two critical points. This occurs with two primaries of $k=1$ in the presence of symmetry breaking, or with three such primaries in the absence of symmetry breaking.

\subsubsection{The first example}

We consider first the case of two primaries with symmetry breaking. We denote the positions of the primaries as $z_{1}$ and $z_{2}$ and we continue to assume that the symmetry breaking parameter $c$ is positive. As long as $z_{1}-z_{2}$ is not real, there is a symmetric choice of basic integration cycles $\mathcal{C}_{a}, a=1,2$ : rays which start at $z_{a}$ and are parallel to the positive real axis. The difference $\mathcal{C}_{1}-\mathcal{C}_{2}=\mathcal{C}_{12}$ is a segment from $z_{1}$ to $z_{2}$. See figure 9 . Any two of these three cycles can be thimbles, depending on the relative values of $c$ and $z_{1}-z_{2}$. 


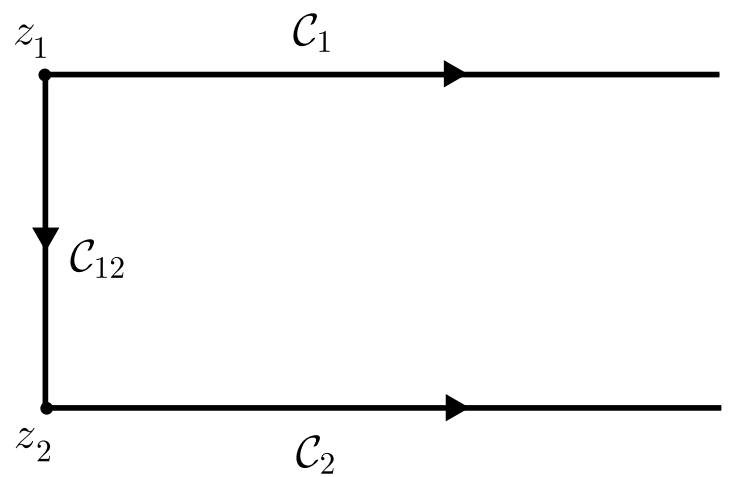

Figure 9: The cycles $\mathcal{C}_{1}, \mathcal{C}_{2}$ and $\mathcal{C}_{12}$.

Since there are always only two thimbles, it is never the case that all three of $\mathcal{C}_{1}, \mathcal{C}_{2}$, and $\mathcal{C}_{12}$ are thimbles.

In this simple example, we already have the basic ingredients of the general braid group representation. The elementary move is to exchange the two $z_{a}$, either clockwise or counterclockwise. We start with a configuration in which the $z_{a}$ have distinct imaginary parts, so that the $\mathcal{C}_{a}$ are well-defined. We may as well take the real parts of the $z_{a}$ to be zero. We want to define the branches of $\mathcal{W}$ along $\mathcal{C}_{1}, \mathcal{C}_{2}$, and $\mathcal{C}_{12}$ so that it is true that $\mathcal{C}_{12}=\mathcal{C}_{1}-\mathcal{C}_{2}$. Picking any branch of $\mathcal{W}$ on $\mathcal{C}_{12}$, we define $\mathcal{W}$ on $\mathcal{C}_{1}$ and on $\mathcal{C}_{2}$ so that at the unique point where $\mathcal{C}_{1}$ intersects $\mathcal{C}_{12}$ or where $\mathcal{C}_{2}$ intersects $\mathcal{C}_{12}$, the definitions agree. This will ensure that $\mathcal{C}_{12}=\mathcal{C}_{1}-\mathcal{C}_{2}$. Although $\mathcal{C}_{1}$ and $\mathcal{C}_{2}$ do not intersect, we can deform them slightly so that they meet at a reference point far to the right, and then the two values of $\mathcal{W}$ will agree at this reference point. (This is ensured by the fact that one can define $\mathcal{W}$ to be single-valued in the semi-infinite rectangle bounded by $\mathcal{C}_{1}, \mathcal{C}_{2}$, and $\mathcal{C}_{12}$.)

Suppose that $\operatorname{Im}\left(z_{1}-z_{2}\right)>0$. Then we can cross to $\operatorname{Im}\left(z_{1}-z_{2}\right)<0$ in two ways, with $z_{1}$ passing either to the left or to the right of $z_{2}$. The two operations are inverses, so it will suffice to consider one in detail. If $z_{1}$ passes to the right of $z_{2}$, then $\mathcal{C}_{1}$ evolves continuously as a ray parallel to the positive real axis. On the other hand, $\mathcal{C}_{2}$ does not. We can use instead $\mathcal{C}_{12}$, defined continuously as a segment from $z_{1}$ to $z_{2}$. In the basis of $\mathcal{C}_{1}$ and $\mathcal{C}_{12}$, this braiding move is diagonal: we only have to keep track of the branches of $\mathcal{W}$ and orientation of cycles. We want to express the final result in the basis of $\mathcal{C}_{1}$ and $\mathcal{C}_{2}$.

In the half-braiding, $\mathcal{C}_{1}$ is multiplied by a factor of $q^{-\frac{1}{4}}: z_{1}$ is transported clockwise around $z_{2}$ and the reference point does not move significantly. 


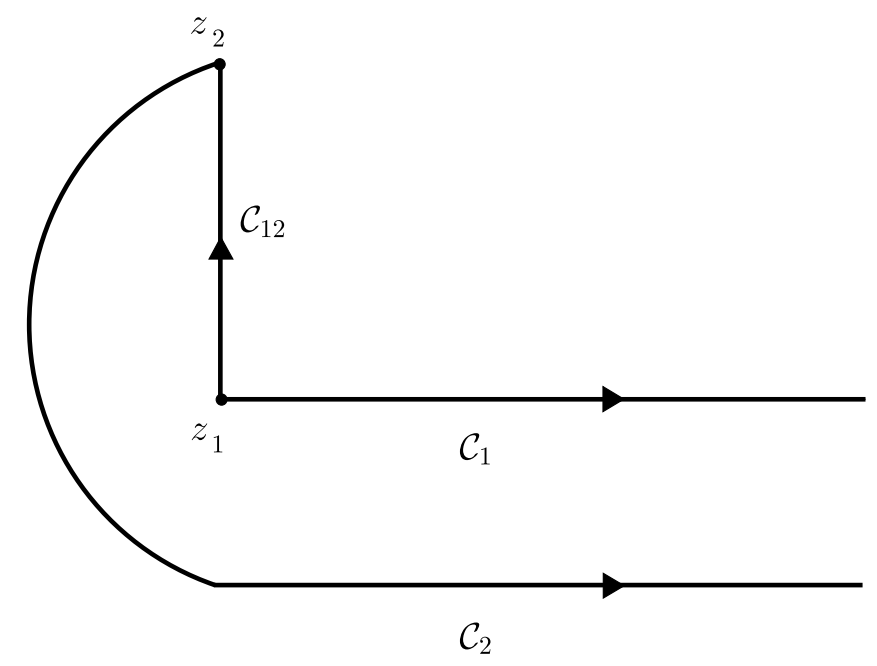

Figure 10: The cycles $\mathcal{C}_{1}, \mathcal{C}_{2}$ and $\mathcal{C}_{12}$ after a braiding operation.

After the half-braiding, we rename $\mathcal{C}_{1}$ as $\mathcal{C}_{2}$ :

$$
\mathcal{C}_{1} \rightarrow q^{-\frac{1}{4}} \mathcal{C}_{2}
$$

On the other hand, $\mathcal{C}_{2}$ becomes the cycle in figure 10. It can be deformed to the sum of three pieces that zigzag to and from $\operatorname{Re} z=\infty$, as in figure 11 . Each of the three pieces is equivalent to a power of $q$ times one of the original cycles $\mathcal{C}_{1}$ and $\mathcal{C}_{2}$. Just as in (6.6), one piece (after again exchanging the labels of $\mathcal{C}_{1}$ and $\left.\mathcal{C}_{2}\right)$ is $q^{-\frac{1}{4}} \mathcal{C}_{2}$. The other two pieces are images of $-q^{-\frac{1}{4}} \mathcal{C}_{2}$ and $q^{-\frac{1}{4}} \mathcal{C}_{1}$ under a deck transformation. Hence, the braiding transformation is

$$
\mathcal{C}_{2} \rightarrow q^{-\frac{1}{4}} \mathcal{C}_{2}-q^{\frac{3}{4}} \mathcal{C}_{2}+q^{\frac{3}{4}} \mathcal{C}_{1}
$$

Note that with these transformation rules,

$$
\mathcal{C}_{12} \rightarrow-q^{\frac{3}{4}} \mathcal{C}_{12}
$$

This is the same result that we found in equation (6.4) (the sign of the exponent is reversed because in deriving (6.8), we braided $z_{1}$ clockwise around $z_{2}$ ); symmetry breaking does not affect the fact that $\mathcal{C}_{12}$ represents the conformal block in which the two degenerate fields fuse to the identity. The braiding matrix has eigenvalues $-q^{\frac{3}{4}}$ and $q^{-\frac{1}{4}}$, which correspond to the two 


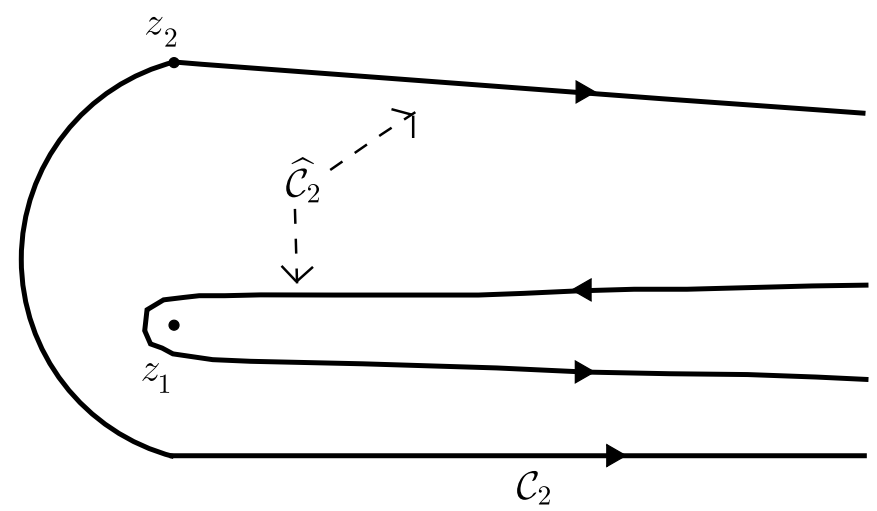

Figure 11: The cycle $\mathcal{C}_{2}$, after the braiding operation, has been copied from figure 10: it starts at $z_{2}$, curves below $z_{1}$ to the left and then goes to $\operatorname{Re} z=\infty$. It is equivalent in homology to a zigzag cycle, labeled $\widehat{\mathcal{C}}_{2}$ in the figure, which, starting at $z_{2}$, heads directly to $\operatorname{Re} z=\infty$ before doubling back around $z_{1}$ and returning to $\operatorname{Re} z=\infty$. Thus $\widehat{\mathcal{C}}_{2}$ is the sum of three pieces, each of which heads to or from $\operatorname{Re} z=\infty$; each piece is equivalent to a power of $q$ times an elementary cycle $\mathcal{C}_{1}$ or $\mathcal{C}_{2}$ (a ray starting at $z_{1}$ or $z_{2}$ and parallel to the positive $z$ axis).

possible fusion channels. The linear combination $\mathcal{C}_{1}+q^{-1} \mathcal{C}_{2}$ transforms as

$$
\mathcal{C}_{1}+q^{-1} \mathcal{C}_{2} \rightarrow q^{-\frac{1}{4}}\left(\mathcal{C}_{1}+q^{-1} \mathcal{C}_{2}\right)
$$

and hence it represents the fusion in the channel of momentum $-b$.

Now, other choices of normalization of $\mathcal{C}_{1}$ and $\mathcal{C}_{2}$ may occur more naturally in various situations. If we change the relative normalization between $\mathcal{C}_{1}$ and $\mathcal{C}_{2}$, say by setting $\widetilde{\mathcal{C}_{1}}=q^{-s / 2} \mathcal{C}_{1}$ and $\widetilde{\mathcal{C}_{2}}=q^{s / 2} \mathcal{C}_{2}$, we can get braiding formulae

$$
B_{12}: \widetilde{\mathcal{C}}_{1} \rightarrow q^{-s-\frac{1}{4}} \widetilde{\mathcal{C}_{2}} \quad \widetilde{\mathcal{C}}_{2} \rightarrow\left(q^{-\frac{1}{4}}-q^{\frac{3}{4}}\right) \widetilde{\mathcal{C}_{2}}+q^{s+\frac{3}{4}} \widetilde{\mathcal{C}_{1}}
$$

We will find the choice $s=-\frac{1}{2}$ to be useful momentarily, so whenever we write $\widetilde{\mathcal{C}_{a}}$ we assume that choice of $s$.

The behavior of the $\mathcal{C}_{a}$ as a function of $c\left(z_{1}-z_{2}\right)$ is depicted in figure 12 . The $\mathcal{C}_{a}$ fail to be well-defined when $c\left(z_{1}-z_{2}\right)$ is real. In this very simple example, the integral over the $\mathcal{C}_{a}$ can be expressed explicitly in terms of 


$$
\mathcal{C}_{1}, \mathcal{C}_{2}
$$

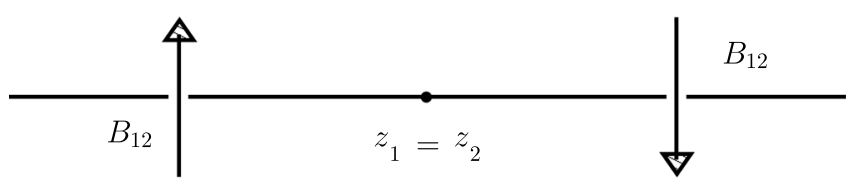

Figure 12: The cycles $\left(\mathcal{C}_{1}, \mathcal{C}_{2}\right)$ (defined as rays in the direction of $\operatorname{Re} c z$ that start at $z=z_{1}$ or $z_{2}$ ) provide a local basis for homology, but the definition of the cycles jumps by the braiding matrix $B_{12}$ across the line on which $Z=c\left(z_{1}-z_{2}\right)$ is real.

familiar functions:

$$
\begin{aligned}
\mathcal{I}_{a} & =\oint_{\mathcal{C}_{a}}\left(w-z_{1}\right)^{\frac{1}{b^{2}}}\left(w-z_{2}\right)^{\frac{1}{b^{2}}}\left(z_{1}-z_{2}\right)^{-\frac{1}{2 b^{2}}} \mathrm{e}^{\frac{c z_{1}}{2}+\frac{c z_{2}}{2}-c w} d w \\
& =c^{-1-\frac{3}{2 b^{2}}} \oint_{\mathcal{C}_{a}}(W-Z)^{\frac{1}{b^{2}}} W^{\frac{1}{b^{2}}} Z^{-\frac{1}{2 b^{2}}} \mathrm{e}^{\frac{Z}{2}-W} d W .
\end{aligned}
$$

Here we defined $W=c\left(w-z_{2}\right)$ and $Z=c\left(z_{1}-z_{2}\right)$. This integral can be explicitly written in terms of Bessel functions. The integrals over $\mathcal{C}_{1}$ and $\mathcal{C}_{2}$ give a basis of Bessel functions with specific asymptotic behavior at large $Z$, and the braiding matrix $B$ captures the Stokes phenomena of Bessel functions:

$$
\begin{aligned}
& \mathcal{I}_{1}=\frac{1}{\sqrt{\pi} c^{1+\frac{3}{2 b^{2}}}} Z^{\frac{1}{2 b^{2}}+\frac{1}{2}} \Gamma\left(1+\frac{1}{b^{2}}\right) K_{\frac{1}{2}+\frac{1}{b^{2}}}\left(\frac{Z}{2}\right) \\
& \mathcal{I}_{2}=\frac{q^{1 / 4}}{\sqrt{\pi} c^{1+\frac{3}{2 b^{2}}}} Z^{\frac{1}{2 b^{2}}+\frac{1}{2}} \Gamma\left(1+\frac{1}{b^{2}}\right) K_{\frac{1}{2}+\frac{1}{b^{2}}}\left(-\frac{Z}{2}\right)
\end{aligned}
$$

In the large $Z$ limit, the integral along $\mathcal{C}_{a}$ is controlled by the region where $w$ is close to $z_{a}$. It is useful to pick a branch of the logarithms such that for $w$ near $z_{1}$ (or $z_{2}$ ), the sum of the logarithms in the superpotential approaches $\frac{1}{4} \log \left(z_{1}-z_{2}\right)^{2}$. This is the same as the change in normalization between $\mathcal{C}_{a}$ and $\widetilde{\mathcal{C}_{a}}$ for $s=-\frac{1}{2}$. This basis of $\widetilde{\mathcal{C}}_{a}$ will be useful whenever we are at strong symmetry breaking. In this basis

$$
B_{12}:=\widetilde{\mathcal{C}}_{1} \rightarrow q^{\frac{1}{4}} \widetilde{\mathcal{C}_{2}} \quad \widetilde{\mathcal{C}_{2}} \rightarrow\left(q^{-\frac{1}{4}}-q^{\frac{3}{4}}\right) \widetilde{\mathcal{C}_{2}}+q^{\frac{1}{4}} \widetilde{\mathcal{C}_{1}} .
$$

So far, we have analyzed this problem using cycles $\mathcal{C}_{1}, \mathcal{C}_{2}$, and $\mathcal{C}_{12}$ that are visible by inspection. Let us compare these cycles with the thimbles. 


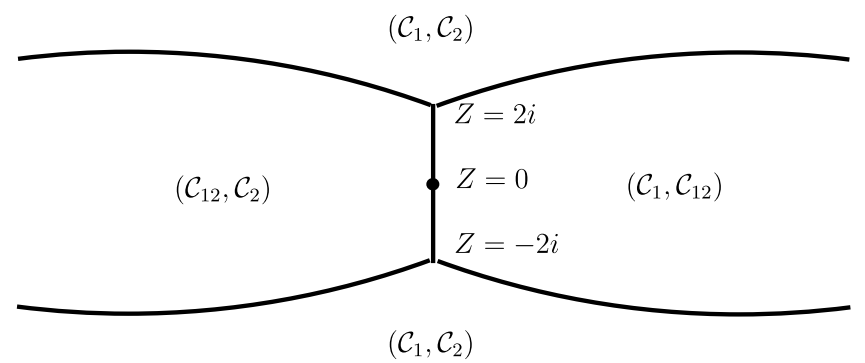

Figure 13: The pattern of Stokes walls and the bases of thimbles in the $c\left(z_{1}-z_{2}\right)$ plane.

The Bethe equation with two $z$ 's and one $w$ is

$$
\frac{1}{w-z_{1}}+\frac{1}{w-z_{2}}=c
$$

and it has two solutions.

There are two regimes of interest. If $|Z| \gg 1$, then the critical points are approximately $w=z_{a}+1 / c, a=1,2$. For each of the two critical points, assuming that $c>0$ and $\operatorname{Re} Z=0$, both $w-z_{1}$ and $w-z_{2}$ have positive real part and it is natural in defining $\mathcal{W}$ to pick branches of $\log \left(w-z_{1}\right)$ and $\log \left(w-z_{2}\right)$ such that the imaginary parts are bounded by $\pm \pi / 2$. The thimbles defined this way coincide with the $\widetilde{\mathcal{C}}_{a}$ we defined above (and not with the $\mathcal{C}_{a}$ ). The advantage of this choice is that it extends naturally to the general case of many fields with symmetry breaking, which we will treat in Section 6.5. If $|Z| \ll 1$, then we have approximate critical points $w=\frac{1}{2}\left(z_{1}+z_{2}\right)$ and $w=2 / c$. The first critical point sits between the two $z_{a}$, and the associated thimble is $\mathcal{C}_{12}$. The second critical point is associated to $\mathcal{C}_{1}$ if $\operatorname{Re} Z>0, \mathcal{C}_{2}$ otherwise.

We depict the Stokes walls for the system of thimbles in figure 13. There are regions in parameter space where the basis of thimbles consists of any two of $\mathcal{C}_{1}, \mathcal{C}_{2}$ and $\mathcal{C}_{12}$, up to a choice of branches of the superpotential. Pairs of regions meet along Stokes walls, and triples of regions meet at the points $Z= \pm 2$ i where the two critical points coincide. This is a general feature; in a generic problem of this type, there will always be loci of complex codimension 1 where two critical points coincide. In a plane transverse to such a locus, $\mathcal{W}$ can be modeled by a simple cubic function $\mathcal{W}=w^{3}+\delta w$ of one variable $w$, with a parameter $\delta$; this function has two critical points that coincide for $\delta=0$, where three Stokes walls meet. The local Stokes behavior is universal; it corresponds to the behavior of the Airy function. 


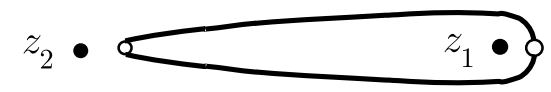

Figure 14: The two Morse flows from the critical point (empty dot) near $z_{2}$ to the critical point (empty dot) near $z_{1}$. The flows occur at slightly different values of $\operatorname{Im} Z$.

As we explained in our general discussion of Section 5.1, the asymptotic behavior of integrals for $b \rightarrow 0$ is clearest in the basis of thimbles. For the present problem, figure 13 captures the relevant information. There are four regions: the upper and lower regions correspond to the system of thimbles we saw in the $|Z| \gg 1$ limit, the two intermediate regions to the system of thimbles which we saw in the $|Z| \ll 1$ limit.

Formulae (6.10) or (6.13) describe the braiding matrix $B_{12}$ that compares the region at the top of figure 13 , where the thimbles are $\mathcal{C}_{1}$ and $\mathcal{C}_{2}$, to the region at the bottom, where again the thimbles are $\mathcal{C}_{1}$ and $\mathcal{C}_{2}$. We did this computation by inspection, not by analyzing the Stokes lines. In deriving (6.13), we assumed that $z_{1}$ moves half-way around $z_{2}$ in a clockwise direction; this is equivalent to moving from the top to the bottom of figure 13 with $\operatorname{Re} Z>0$. From figure 13, we see that in this process, we will cross two Stokes lines. This means that in the basis of thimbles, the braiding matrix $B_{12}$ "decomposes" into a sequence of two elementary moves, each associated to one Stokes line. Each elementary move involves a gradient flow in which the Bethe root $w$ flows from a critical point just to the right of $z_{2}$ to a critical point just to the right of $z_{1}$. There are two such flows, differing by whether $w$ passes above or below $z_{1}$. The two flows, which occur at slightly different values of $\operatorname{Im} Z$, are sketched in figure 14 . In the formula $B_{12} \widetilde{C}_{2}=\left(q^{-1 / 4}-q^{3 / 4}\right) \widetilde{C}_{2}+q^{1 / 4} \widetilde{C}_{1}$ of equation (6.13), the term $q^{1 / 4} \widetilde{C}_{1}$ on the right-hand side is the contribution of the formal monodromy alone, whereas the other two terms are contributions from the two gradient flow solutions.

In the small $Z$ region, braiding around $Z=0$ is represented in the basis of thimbles by a triangular matrix: $\mathcal{C}_{12}$ is an eigenvector. There is a general reason for this fact; the integral over $\mathcal{C}_{12}$ is smaller than the integral over any other cycle either in the $Z \rightarrow 0$ limit, or in the $b \rightarrow 0$ limit. In either limit, the integral over $\mathcal{C}_{12}$ is controlled by the usual saddle point approximation, and the critical point associated to $\mathcal{C}_{12}$ is the one at which the Morse function is the smallest. So $\mathcal{C}_{12}$ must be an eigenvector of the monodromy. With any number of $z$ 's and w's, the set of thimbles which has one $w$ in between a given pair of very close $z_{a}$ span the "small" eigenspace of conformal blocks where the two degenerate fields of momentum $-\frac{1}{2 b}$ fuse to the identity. 


\subsubsection{The second example}

The second example with two critical points arises if there are three singular monopoles of minimum charge and a single Bethe root $w$. Placing the singular monopoles at $z_{1}, z_{2}, z_{3}$, there are three obvious possible integration cycles: a straight path connecting $z_{a}$ to $z_{b}$ for any $a, b$. We can pick the branch of the superpotential in such a way that these three cycles add to zero.

This definition makes sense if the three points are not aligned. The parameter space of $z_{a}$ is then split into two halves: either $z_{1}, z_{2}$ and $z_{3}$ form a triangle with positive orientation, or they form a triangle with negative orientation. We will denote the three natural cycles in either case as $\mathcal{C}_{a b}^{ \pm}$. So

$$
\mathcal{C}_{12}^{+}+\mathcal{C}_{23}^{+}+\mathcal{C}_{31}^{+}=0 \quad \mathcal{C}_{12}^{-}+\mathcal{C}_{23}^{-}+\mathcal{C}_{31}^{-}=0
$$

but the two bases are related in an interesting way across the loci where the $z_{a}$ are collinear.

There are three such loci, where one of the three $z_{a}$ passes between the other two. For example, if $z_{2}$ passes between $z_{1}$ and $z_{3}, \mathcal{C}_{12}^{+}$and $\mathcal{C}_{23}^{+}$will be related to $\mathcal{C}_{12}^{-}$and $\mathcal{C}_{23}^{-}$simply by a change of branch of $\mathcal{W}$, while the transformation of $\mathcal{C}_{13}^{+}$then follows from (6.15).

The Bethe equation

$$
\frac{1}{w-z_{1}}+\frac{1}{w-z_{2}}+\frac{1}{w-z_{3}}=0
$$

is equivalent to a quadratic equation for $w$, so it has two solutions, corresponding to two independent thimbles. The thimbles are equivalent to two of the paths joining a pair of $z$ 's, but which pairs appear depends on the choice of the $z$ 's. If the $z$ 's are collinear, then the Bethe roots are located in the segments between adjacent $z$ 's, and the thimbles coincide with those segments. For example, if $z_{2}$ is between $z_{1}$ and $z_{3}$, the two thimbles are $\mathcal{C}_{12}^{ \pm}$ and $\mathcal{C}_{23}^{ \pm}$, up to powers of $q$.

As usual, the thimble joining $z_{a}$ and $z_{b}$ corresponds to the conformal block where the corresponding two degenerate fields fuse to the identity. The relations (6.15) correspond to the elementary "skein relation" between the three different ways to fuse two of the three primary fields to the identity. In other words, whenever $z_{a}$ and $z_{b}$ are close together, there is a thimble which joins them and is an eigenvector of the braiding of $z_{a}$ and $z_{b}$, and that braiding matrix is triangular. 


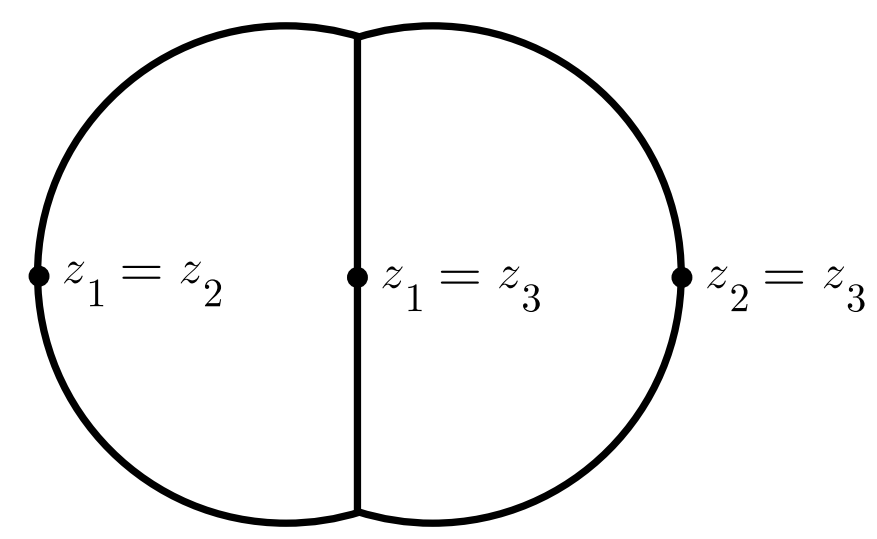

Figure 15: The pattern of Stokes walls in the space of shapes of the triangle with vertices $z_{1}, z_{2}, z_{3}$.

We depict the Stokes walls in figure 15. The integrals $I_{a b}$ can be readily evaluated in terms of hypergeometric functions.

\subsection{A final example}

There is one more example that is both instructive and relevant to understanding the general picture. This is the case of two $z_{a}$ of charge 1 , accompanied by two $w_{i}$. This can only happen in the presence of complex symmetry breaking. It is easy to see that the Bethe equations (3.37) have only a unique solution. $Q$ and $K$ are both of degree 2 , so $P$ is of degree 0 and can be set to 1 . This leads to linear equations that uniquely determine the coefficients in $Q$. Since the solution of the Bethe equations is unique, there is only one conformal block and the monodromy in braiding the $z_{a}$ can only be multiplication by a function of $q$.

If $z_{1}$, and $z_{2}$ are well-separated, i.e., $c\left(z_{1}-z_{2}\right)$ has large absolute value, then the solution of the Bethe equations is easily described: $w_{1} \sim z_{1}+1 / c$ and $w_{2} \sim z_{2}+1 / c$. There is an obvious integration cycle, with $w_{1}$ and $w_{2}$ integrated respectively over the rays from $z_{1}$ and $z_{2}$ to infinity in the $c$ direction; these rays were labeled $\mathcal{C}_{1}$ and $\mathcal{C}_{2}$ in figure 9 . We can denote this cycle as $\mathcal{C}=\mathcal{C}_{1} \times \mathcal{C}_{2}$.

The unique thimble for the problem is a small deformation of $\mathcal{C}$ if the imaginary part of $Z=c\left(z_{1}-z_{2}\right)$ is large. As we deform $Z$, the unique thimble will deform continuously. Here Morse theory is rather useful: it is rather tricky to verify by hand that $\mathcal{C}$ goes back to itself under braiding of $z_{1}$ and $z_{2}$, as it requires a contour deformation which does not keep $\mathcal{C}$ in a 
simple product form. But the evolution of the thimble provides us implicitly with such a deformation. It is likewise far from obvious at first sight that the conformal block produced by the contour integral over $w_{1}$ and $w_{2}$ will be a simple function with abelian monodromy in the $Z$-plane. Nevertheless, this must be the case.

The effect of braiding is easily computed at large $c$, by looking at the saddle point estimate for the integral. At the saddle, $w_{1} \sim z_{1}+1 / c$ and $w_{2} \sim z_{2}+1 / c$ remains true along the whole braiding, as long as it is executed at large $|Z|$. The Hessian of $\mathcal{W}$ is close to a large, $Z$-independent, multiple $\left(\sim|c|^{2}\right)$ of the identity matrix, so the braiding phase at large $Z$ is controlled by the value of $\exp \left(\mathcal{W} / b^{2}\right)$ at the critical point. The relevant factors are

$$
\left(z_{1}-z_{2}\right)^{-b^{-2} / 2}\left(w_{1}-z_{2}\right)^{b^{-2}}\left(w_{2}-z_{1}\right)^{b^{-2}}\left(w_{1}-w_{2}\right)^{-2 b^{-2}} \sim\left(z_{1}-z_{2}\right)^{-b^{-2} / 2} .
$$

Hence, we recover the same braiding phase as in a setup with two $z_{a}$ of charge 1 and no $w_{i}$.

This last statement will be part of the input for constructing an effective abelian description in Section 6.7. In an abelian theory, one would expect that the braiding of two objects depends only on the product of their charges. So braiding of two objects both of charge -1 ( $z$ 's unaccompanied by $w$ 's) or two objects both of charge 1 ( $z$ 's accompanied by $w$ 's) gives the same result. Braiding of an object of charge 1 with an object of charge -1 is less simple, since nontrivial gradient flows enter the picture, as we saw in Section 6.3.1.

\subsection{General picture with symmetry breaking}

In this section, we develop a general picture with symmetry breaking. We take the symmetry breaking parameter $c$ to be real and positive; we consider any number of singular monopoles at positions $z_{a}, a=1, \ldots, d$, and any number of Bethe roots $w_{i}, i=1, \ldots, q$. At first we will set all the charges $k_{a}$ to 1 .

We can produce a basis of integration cycles by hand. We assume that the imaginary parts of the $z_{a}$ are distinct, and ordered so that $\operatorname{Im}\left(z_{a}-z_{a^{\prime}}\right)>0$ if $a^{\prime}>a$. We define the rays $\mathcal{C}_{a}$ which start at $z_{a}$ and are parallel to the positive real axis.

Our integration cycles will be products $\mathcal{C}_{a_{1} a_{2} \cdots a_{q}}:=\mathcal{C}_{a_{1}} \times \mathcal{C}_{a_{2}} \times \cdots \times \mathcal{C}_{a_{q}}$ for every subset of $q$ distinct $z$ 's. This gives $\left(\begin{array}{l}d \\ q\end{array}\right)$ integration cycles, which 
in the region of large $c$ can be interpreted as thimbles. Indeed the Bethe equations

$$
\sum_{a} \frac{1}{w_{i}-z_{a}}=c+\sum_{j \neq i} \frac{2}{w_{i}-w_{j}}
$$

have approximate solutions for large $c$ with each $w_{i}$ equal approximately to $z_{a_{i}}+1 / c$, with $a_{i} \neq a_{j}$ for $i \neq j$. The corresponding thimbles are precisely the $\mathcal{C}_{a_{1} a_{2} \ldots a_{q}}$. Summing over all $q$, we get $2^{d}$ critical points or conformal blocks in all, as expected.

The braid group representation is rather simple in this basis. We let the $z_{a}$ move around in the complex plane. The Stokes walls are approximately at the locus where the real parts of two $z_{a}$ coincide. Morse flows will occur if and only if a $z_{a}$ unaccompanied by a $w_{i}$ passes to the right of a $z_{a^{\prime}}$ accompanied by a $w_{i}$. The resulting behavior involves only the two $z$ 's that are crossing and is precisely what we analyzed in detail in Section 6.3.1. The braiding matrix when $z_{a}$ crosses $z_{a^{\prime}}$ acts non-trivially only on $\mathcal{C}_{a}$ and $\mathcal{C}_{a^{\prime}}$ (and any cycle $\mathcal{C}_{a_{1} a_{2} \ldots a_{q}}$ that contains one or both of these), and takes the same form as (6.13). As in figure 14, the braiding matrix for this process can be interpreted as resulting from a pair of Morse theory flows. It is tedious, but elementary, to check that the braid group relations are satisfied, as they should be.

\subsubsection{Degenerate fields of any charge}

Now, still with complex symmetry breaking, we will relax the constraint that all $k_{a}$ equal 1 . First, we can consider a single $z$ of charge $k$, with $q$ Bethe roots. The Bethe equations only have solutions if $k \geq q$. (This is clear from equation (3.37) for the opers.) Actually, according to the theory of Bethe equations, the solution is unique, for given $q$, and corresponds to a unique thimble or integration cycle.

Indeed, it is easy to describe this unique possible integration cycle $\mathcal{C}^{(q)}$ : one integrates each $w_{i}$ from $z$ to $+\infty$, along distinct, nonintersecting paths, as in figure 16.

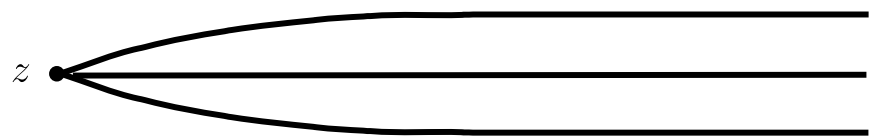

Figure 16: An integration cycle for one singular monopole of nonminimal charge. 

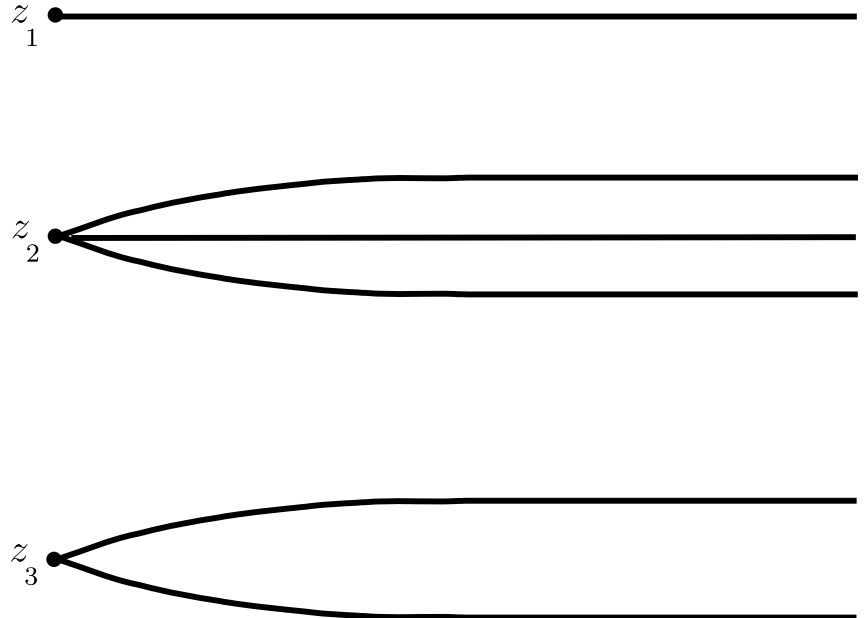

Figure 17: An integration cycle for several singular monopoles of nonminimal charge. To $z_{a}$ we attach $q_{a}$ of the $w$ 's, for suitable $q_{a}$. In the picture, the $q_{a}$ are 1,3 , and 2 .

We can then immediately describe a basis of integration cycles in the most general case, with any number of $z_{a}$ of charge $k_{a}$, and the number of $w_{i}$ being $q \leq \sum_{a} k_{a}$. We get a unique cycle for each way to decompose $q=\sum_{a} q_{a}$ with $q_{a} \leq k_{a}$ : for each $a$, we integrate $q_{a}$ of the $w_{i}$ from $z_{a}$ to $+\infty$, along distinct, nonintersecting paths, as in figure 17. This gives an integration cycle $\prod_{a} \mathcal{C}_{a}^{\left(q_{a}\right)}$.

At strong symmetry breaking (or equivalently, if the $z_{a}$ are well separated along the imaginary axis), these integration cycles correspond to the thimbles associated to the unique solutions of the Bethe equations with $q_{a}$ of the $w_{i}$ near $z_{a}$. There is no obstruction, in principle, to derive the braid group representation in this basis.

For a homological approach - essentially corresponding to the free-field realization - to the construction of braid group representations for any $k_{a}$, although without symmetry breaking, see [48].

\subsection{Turning off symmetry breaking}

Since symmetry breaking is so useful in simplifying our analysis, the question arises of verifying that symmetry breaking does not affect the values of knot or link invariants. 
The description of integration cycles in the previous section is applicable as long as the symmetry breaking parameter $c$ is nonzero. But for $c \rightarrow 0$, we lose some integration cycles, as the $w_{i}$ cannot go to infinity any more. In terms of critical points of $\mathcal{W}$, the behavior for small $c$ is easy to describe. For small $c$, for each solution of the Bethe equations, the Bethe roots split naturally into two subsets. Some number $q_{0}$ of Bethe roots, which we call $w_{i}^{(0)}, i=1, \ldots, q_{0}$, remain of order 1 in the limit $c \rightarrow 0$, while the remaining $q_{\infty}$ Bethe roots, which we call $w_{j}^{(\infty)}, j=1, \ldots, q_{\infty}$, are of order $c^{-1}$.

In this situation, the Bethe equations for the $w_{i}^{(0)}$ are well approximated by the Bethe equations for that number $w$ 's in the absence of symmetry breaking. On the other hand, the Bethe equations for the $w_{j}^{(\infty)}$ are well approximated by the Bethe equations for that number of $w$ 's, in the presence of a single $z$ of charge $k_{\text {eff }}=\sum_{a} k_{a}-2 q_{0}$. Equation (3.22) ensures that $k_{\text {eff }}$ is non-negative, so in fact

$$
0 \leq k_{\mathrm{eff}} \leq \sum_{a} k_{a}
$$

As we have discussed in Section 6.5.1, the Bethe equations for the $w_{i}^{(\infty)}$ have a single solution if $k_{\text {eff }} \geq q_{\infty}$; otherwise, they have no solutions. Summing over all decompositions $q=q_{0}+q_{\infty}$ and all solutions of the Bethe equations for the $w_{i}^{(0)}$, and finally over all possible values of $q$, one gets the expected number $\prod_{a=1}^{d}\left(k_{a}+1\right)$ of solutions of the Bethe equations.

The counting can be carried out as follows, in terms of Wilson operators of the dual $S U(2)$ gauge theory. The singular monopoles of charge $k_{a}$ correspond to representations $R_{a}$ of $S U(2)$ of spin $k_{a} / 2$ and dimension $k_{a}+1$. The tensor product $R=\otimes_{a} R_{a}$ has dimension $\prod_{a}\left(k_{a}+1\right)$. This representation can be decomposed as a direct sum of $S U(2)$ modules of spin $k_{\text {eff }} / 2$ (where $k_{\text {eff }}$ is bounded by $0 \leq k_{\text {eff }} \leq \sum_{a} k_{a}$, just as in (6.19)). The states that transform with this spin are as numerous as the solutions of the Bethe equations with $\sum_{a} k_{a}-2 q_{0}=k_{\text {eff }}$ (and all possible values of $q$ ).

Now consider braiding of the $z_{a}$. For sufficiently small $c$, when one crosses a Stokes wall, there are Morse flows in which $q_{\infty}$ becomes smaller, but no such flows in which $q_{\infty}$ becomes larger. The reason for this is that for $c \rightarrow 0$, the values of the Morse function $h=\operatorname{Re} \mathcal{W}$ at a critical point are greater the greater is $q_{\infty}$. (This is because some contributions to $h$ are of order $\ln (1 /|c|$ ) for $c \rightarrow 0$. With the help of (6.19), one can show that the coefficient of $\ln (1 /|c|)$ is an increasing function of $q_{\infty}$.) As a result, decomposing the space $\mathcal{H}$ of physical states according to the value of $q_{\infty}$, the monodromy 
matrix is block triangular:

$$
B \sim\left(\begin{array}{lllll}
* & * & * & * & * \\
* & * & * & * & * \\
0 & 0 & * & * & * \\
0 & 0 & * & * & * \\
0 & 0 & 0 & 0 & *
\end{array}\right)
$$

The diagonal blocks (which are of rank 2,2, and 1 in the example given) are the monodromy representations that one would have in the absence of symmetry breaking for given $q_{0}=q-q_{\infty}$.

To the extent that one can compute knot or link invariants by taking traces of braid group representations, the off-diagonal blocks in (6.20) are not important as they do not contribute to traces. Actually, to compute the Jones polynomial and related invariants of knots and links, one needs in addition to the braid group representations an additional "fusion" operation in which a pair of $z_{a}$ of the same charge is created or annihilated. The additional information that we need to ensure that knot invariants are unaffected by symmetry breaking and do not change upon setting $c=0$ is that fusion never involves creating or annihilating any $w$ 's at infinity. This is natural because of the local nature of the fusion operation.

\subsubsection{A clarification}

A careful reader might notice a small sleight of hand in this derivation. The inequality $k_{\text {eff }} \geq 0$ was deduced from (3.22), but the original derivation of (3.22) was based on picking $Q$ to have a smaller degree than $P$. The Bethe equations at $c=0$ certainly have solutions in which this is not the case. Why are we entitled to restrict to this case?

Suppose that at $c=0$, we find a pair $P_{0}, Q_{0}$ obeying the oper condition

$$
P \frac{d Q}{d z}-\frac{d P}{d z} Q=K(z) .
$$

Now suppose that we turn on very weak symmetry breaking. The oper equation becomes

$$
P \frac{d Q}{d z}-\frac{d P}{d z} Q-c P Q=K(z) .
$$

We hope that as $c$ is turned on, there is a pair $(P(z ; c), Q(z ; c))$ obeying (6.22) and such that $Q$ has an expansion

$$
Q(z ; c)=Q_{0}+c Q_{1}+c^{2} Q_{2}+\cdots .
$$


This will ensure that the $Q(z ; c)$ has roots that approach the roots of $Q_{0}$ as $c \rightarrow 0$, plus possible additional roots that go to infinity for $c \rightarrow 0$. One might expect that $P$ would have an expansion of the same form, but this is not the case. The degrees $p$ and $q$ of polynomials $P, Q$ obeying (6.21) satisfy $p+q=k+1$, but as soon as $c \neq 0$, the relation becomes $p+q=k$. So the degree of $P$ must drop as soon as $c \neq 0$. The way that this happens is that the expansion for $P$ is actually

$$
P(z ; c)=c^{-1} P_{-1}+\widetilde{P}_{0}+c P_{1}+\cdots
$$

where we write $\widetilde{P}_{0}$ for the coefficient of $c^{0}$, as this polynomial does not coincide with $P_{0}$. Plugging (6.23) and (6.24) in (6.22), we learn from the term of order $c^{-1}$ in the equation that $P_{-1}$ is a multiple of $Q_{0}$, and this multiple must be nonzero or else the term of order $c^{0}$ in the equation would force $p+q \geq k+1$. So $p \geq q_{0}$, and this together with $p+q=k$ and $q \geq q_{0}$ implies that $q_{0} \leq k / 2$, as desired.

The moral of the story is that solutions of the Bethe equations for $c=0$ with $q>k / 2$ do exist, but they are unstable to symmetry breaking. Various forms of this statement are known in the literature on integrable systems.

\subsection{Three-dimensional interpretation}

To apply our results to knots and not just to braids, it will help to understand the three-dimensional interpretation of what we have computed so far. We consider knots in $\mathbb{R}^{3}$, so the four-manifold on which we are trying to count solutions of equations (1.1) is $M_{4}=\mathbb{R}^{3} \times \mathbb{R}_{+}$. We describe $\mathbb{R}^{3}$ with Euclidean coordinates $x^{1}, x^{2}, x^{3}$. The adiabatic evolution considered so far has been in the $x^{1}$ direction, while we have combined the other coordinates to a complex variable $z=x^{2}+i x^{3}$. As usual, we take the gauge group to be $G=S O(3)$.

We will focus on the case of strong symmetry breaking. The symmetry breaking involves the choice of an expectation value $\vec{\phi}=\operatorname{diag}(\overrightarrow{\boldsymbol{a}},-\overrightarrow{\boldsymbol{a}})$, where $\overrightarrow{\boldsymbol{a}}$ is a vector in $\mathbb{R}^{3}$. As long as the complex symmetry breaking is nonzero, this vector does not point in the $x^{1}$ direction, that is, the direction that we chose for the adiabatic evolution. Topologically, if the directions are not the same, we may as well think of them as orthogonal: we consider adiabatic evolution in the $x^{1}$ direction, and symmetry breaking with $\vec{c}=$ $-2 \vec{a} / \zeta$ pointing in the positive $x^{2}$ direction. This will be strong complex symmetry breaking with real, positive $c$, in the terminology that we have used so far. 
We will concentrate on the case that the strands have minimum magnetic charge only, and thus are dual to the two-dimensional representation of $S U(2)$. At a generic time, each strand has two possible states: it is or it is not accompanied by a Bethe root $w_{i}$. In the low-energy effective abelian gauge theory, the strand has magnetic charge 1 if accompanied by a Bethe root, and otherwise -1 .

The magnetic charge of a given strand changes when the Bethe root accompanying that strand moves to another strand. In the context of adiabatic evolution, this results from a Morse theory flow in which a Bethe root moves from one strand to another. This happens at a value of $x^{1}$ at which one crosses a Stokes wall. The lesson of Section 6.3.1 is that (in the limit of strong symmetry breaking) one crosses a Stokes wall at a time (that is a value of $x^{1}$ ) at which two strands have the same value of $x^{3}=\operatorname{Im} z$. So the crossing of a Stokes wall occurs when two strands have common values of $x^{1}$ and $x^{3}$, and thus differ only in $x^{2}$. Differently put, this happens when the two strands are separated in the direction of symmetry breaking.

A three-dimensional picture clarifies things (figure 18). When two strands align along the $x^{2}$ direction, a Morse flow can occur. The Morse flow occurs on a time scale fast compared to the adiabatic evolution, so in the adiabatic picture it is essentially instantaneous. The flow involves a Bethe root moving toward the positive $x^{2}$ direction. The flow can only occur between strands of opposite charge, and will allow positive charge to move toward positive $x^{2}$ only.

In general, given any knot, we can usefully project it to the $x^{1}-x^{3}$ plane, and look at it from the negative $x^{2}$ direction. We suppose that the embedding of the knot in $\mathbb{R}^{3}$ is generic enough so that its tangent vector always has a nonzero projection to the $x^{1}-x^{3}$ plane, and moreover so that the projection of the knot to the plane has only simple crossings; finally we will assume that the function $x^{1}$ has only simple maxima and minima along the knot. A simple example of a knot projection is given in figure 19. In such a knot projection, the low-energy abelian description is valid away from crossings, so away from crossings and local maxima and minima, which we discuss in Section 6.7.1, each strand can be labeled by its magnetic charge 1 or -1 . The charges are unchanged at crossings, unless a strand of positive charge passes over a strand of negative charge, in which case a charge exchange process (corresponding in Morse theory to a nontrivial gradient flow) is possible.

Our previous calculations assign a weight to each possible crossing. These weights are just $q^{ \pm 1 / 4}$ if the charges are unchanged at the crossing. When the charge jumps, the weight is $q^{ \pm 1 / 4}-q^{\mp 3 / 4}$. The weights are summarized 

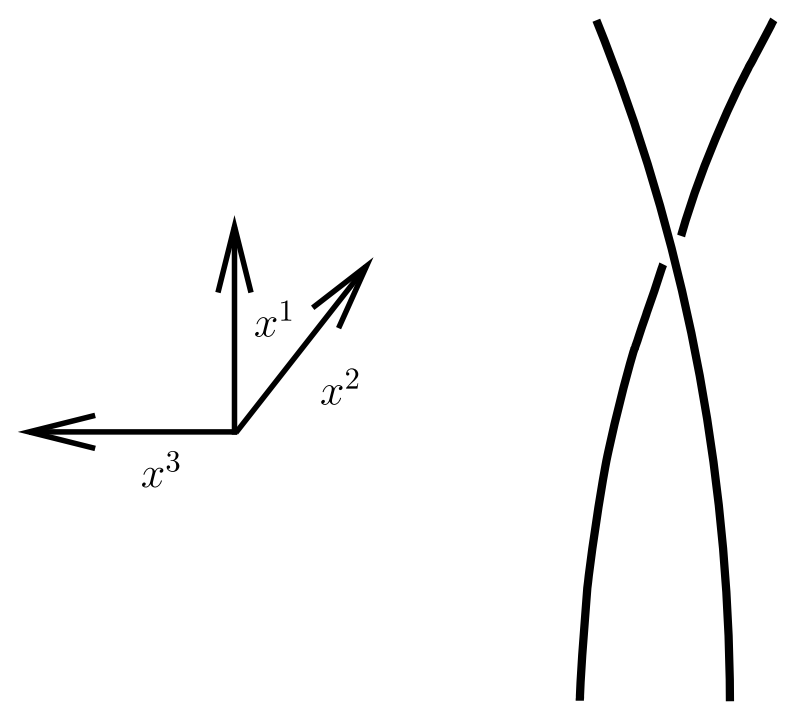

Figure 18: A three-dimensional picture of the process that leads to a Morse theory flow. Just two strands are pictured here. The $x^{1}$ direction is plotted vertically and the $x^{2}$ direction runs into the paper. We are looking at the picture from along the negative $x^{2}$ axis. The coordinates $x^{2}$ and $x^{3}$ combine to a complex variable $z=x^{2}+\mathrm{i} x^{3}$. For a particular choice of the direction of complex symmetry breaking, a nontrivial Morse theory flow can occur only at values of $x^{1}$ at which the two strands have the same value of $x^{3}$ and thus project to the same point in the $x^{1}-x^{3}$ plane. In the language of knot theory, we make a two-dimensional picture by projecting a knot or link to the $x^{1}-x^{3}$ plane. In this projection, there are crossing points, and these are the points at which a nontrivial Morse flow may occur.

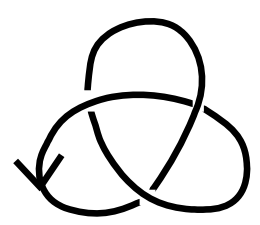

Figure 19: A simple example of a knot projection, with only simple crossings and simple maxima and minima of the height function. This figure also illustrates the fact that the projection to a plane of an oriented knot allows one to define an integer invariant $p=(1 / 2 \pi) \oint d s d \theta / d s$ that is determined by the total change in moving around the knot of the angle $\theta$ defined by the tangent vector to the knot. For the example shown, $p=2 . p$ is the only invariant of a knot projection that can be written as a local integral along the knot. It depends on the choice of projection and is not an invariant of the knot per se. 

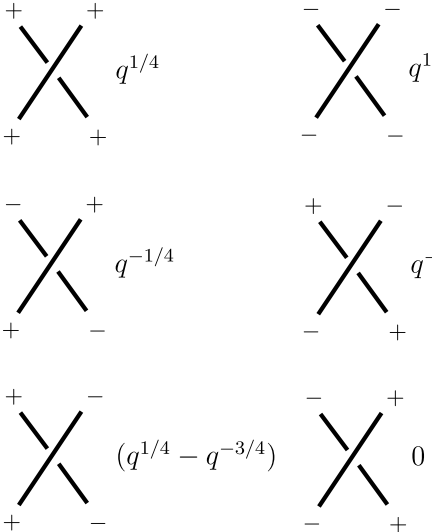
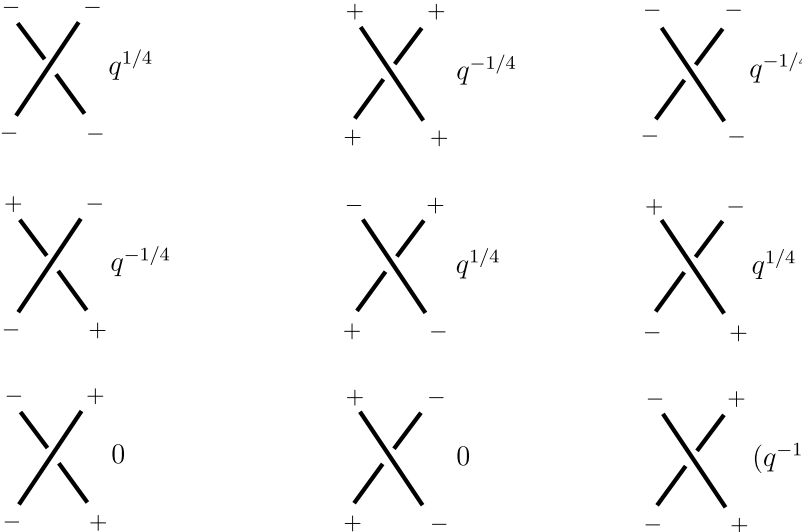

Figure 20: The vertex model assigns the indicated factors to every crossing of two strands. The + and - signs labeling the strands express upward flow of magnetic charge +1 or -1 ; if one turns the picture upside down, the weights remain unchanged, provided one exchanges all + and - labels. Charge can be exchanged between strands, but only when a positive charge flows in from the bottom above a negative charge. If one reflects the picture from left to right, while also replacing $q$ with $q^{-1}$, the weights remain invariant; this reflects the behavior of Chern-Simons theory under reversal of orientation.

in figure 20. We have arrived at a known vertex model representation of the braid group representations associated to the Jones polynomials. See for example the $R$-matrix on page 125 of [49] (where $A$ is our $q^{1 / 4}$ ) or see [50], especially pp. $1777-1778$, or figure 10 of [51].

\subsubsection{Creation and annihilation of strands}

In order to reproduce the knot invariants, we need to understand the loci where the adiabatic approximation is invalid, because two strands are created or annihilated. Although the adiabatic approximation is invalid near such points, the low-energy abelian description remains valid. As an immediate consequence, conservation of charge in the abelian theory makes it clear that only pairs of strands with opposite charge can be created or annihilated.

The map from line operators in a microscopic theory to line operators in an effective low-energy description is akin to the corresponding map for local operators, but it has a little twist: the coefficients are not $c$-numbers, but rather quantum mechanical vector spaces that have to be transported along the line. In the present case, the vector spaces are one-dimensional (in the $U(1)$ theory, an 't Hooft operator has no structure except its charge), 
but we can still get an overall factor from parallel transport. This factor has to be written locally along the loop, and must also be consistent with topological invariance. For a knot without any additional structure there is no topological invariant that can be written as a local integral along the knot, but once one is given a projection of the knot to a plane - in our case the $x^{1}-x^{3}$ plane - there is precisely one such invariant, the total winding number of the tangent vector to the knot (figure 19). This can be written as $(1 / 2 \pi) \oint d s d \theta / d s$, where the knot is parameterized by a variable $s$, and $\theta$ is the angular direction of the tangent vector to the knot in the $x^{1}-x^{3}$ plane. To define the sign of this invariant, one needs an orientation of the knot, which in our case comes from the direction of flow of magnetic charge. When the microscopic $S U(2)$ theory is approximated at long distances as an effective $U(1)$ theory, the effective action for an 't Hooft operator may acquire a term $-\mathrm{i} \eta \oint d s d \theta / d s$, with a universal coefficient $\eta$. If the tangent direction to a knot changes by an angle $\Delta \theta$, this will contribute a factor

$$
\exp (\mathrm{i} \eta \Delta \theta)
$$

In creation or annihilation of a pair of strands, the change in the tangent angle is $\Delta \theta=\pi$ or $-\pi$, depending on whether the positive charge bends to the left or to the right. This effect will associate a universal factor $\exp ( \pm \mathrm{i} \pi \eta)$ to each creation or annihilation event, depending on the direction of flow of charge.

Up to sign, there is a unique choice of $\eta$ that leads to a knot invariant, namely $\exp (\mathrm{i} \pi \eta)=\mp \mathrm{i} q^{1 / 4}$. The choice of sign does not matter, since every knot has an even number of creation and annihilation events; we will take $\exp (\mathrm{i} \pi \eta)=-\mathrm{i} q^{1 / 4}$. The weights for creation and annihilation events with this value of $\eta$ are shown in figure 21 . The value of $\eta$ could possibly be computed directly by studying the four-dimensional gauge theory BPS equations near the abelian limit. The factor of $q^{1 / 4}$ should express the difference between instanton number computed microscopically in the $S O(3)$ theory and instanton number computed in the low-energy $U(1)$ theory. The factor of $\mp i$ should involve a comparison between fermion determinants for $S O(3)$ and for $U(1)$. Globally, the contribution of a given classical solution to the Jones polynomial is proportional to the sign of the fermion determinant, a subtle invariant that may receive contributions from charged modes in the microscopic $S O(3)$ theory; to write this sign as a product of local factors, one apparently must use factors of $i^{ \pm 1}$, with overall signs that depend on how one trivializes the determinant line bundle.

The value of $\eta$ can actually be deduced by combining the information which is available in the abelian description and information available from 


$$
\begin{aligned}
& +\left\lceil-i q^{-1 / 4}+\longdiv { } > i q ^ { - 1 / 4 }\right. \\
& -\left\lfloor+-i q^{1 / 4}-\left(>+i q^{1 / 4}\right.\right.
\end{aligned}
$$

Figure 21: The weights of the vertex model for creation or annihilation of a pair of strands. As in figure 20, the weights are invariant under rotating the picture upside down if one exchanges + and - labels, or under a reflection from left to right if one replaces $q$ by $q^{-1}$.

the conformal block description. When a pair of strands is created or annihilated, we expect them to be fused to the identity. This means that the two nearby strands, located at say $z_{1}$ and $z_{2}$, are accompanied by a Bethe root $w$, and that the integration cycle for this Bethe root is the thimble $\mathcal{C}_{12}$ connecting $z_{1}$ and $z_{2}$. On the other hand, in Section 6.3.1, we also defined integration cycles $\widetilde{\mathcal{C}}_{1}$ and $\widetilde{\mathcal{C}}_{2}$ with the property that the strands at $z_{1}$ and at $z_{2}$ have definite magnetic charges. (For example, in $\widetilde{\mathcal{C}}_{1}$, the Bethe root accompanies $z_{1}$, so the charges of the two strands are respectively 1 and -1 . We order $z_{1}$ and $z_{2}$ in order of decreasing $x^{3}=\operatorname{Im} z$.) The relation among these cycles turned out to be

$$
\mathcal{C}_{12}=\mathcal{C}_{1}-\mathcal{C}_{2}=q^{-1 / 4} \widetilde{\mathcal{C}}_{1}-q^{1 / 4} \widetilde{\mathcal{C}}_{2}
$$

The ratio of the amplitude to create a pair of charges $(1,-1)$ to the amplitude to create a pair of charges $(-1,1)$ is the ratio of the coefficients on the right-hand side of (6.26), or $-q^{-1 / 2}$. On the other hand, in the abelian description, this ratio is $\exp (2 \pi \mathrm{i} \eta)$. So $\exp (\mathrm{i} \pi \eta)=\mp \mathrm{i} q^{-1 / 4}$, as shown in figure 21 .

We have arrived to what is essentially a standard vertex model algorithm for calculating the Jones polynomial: given a knot or a link, pick a projection to the $x^{1}-x^{3}$ plane such that there are only simple crossings and the function $x^{1}$ only has simple maxima and minima. Divide the link into segments separated by the maxima, minima and crossings. Label the segments by \pm and sum over all labelings, weighting each labeling with the product of 

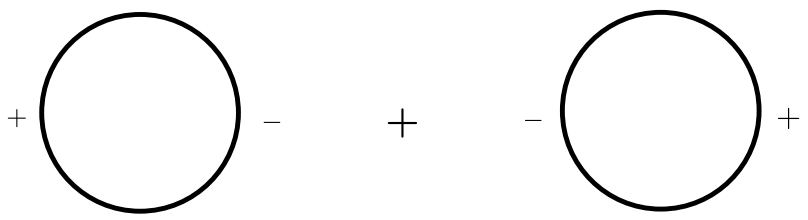

$$
=\quad-q^{1 / 2}-q^{-1 / 2}
$$

Figure 22: An unknot projected to the plane in the most obvious way. There are no crossings, but there is a creation event and an annihilation event. In the vertex model, the invariant for the unknot is computed by summing over all ways to label the two sides of the knot (that is, the segments between crossing, creation, and annihilation events) by charges + or - . Each labeling is weighted by the product of the appropriate local factors. In the present example, only the two choices shown make nonzero contributions, leading at once to the result $-q^{1 / 2}-q^{-1 / 2}$.

the local weights at crossings, maxima, and minima. Some simple examples are given momentarily.

\subsubsection{Some examples and some topological details}

For the simplest example of the use of the vertex model, we compute the expectation value of an unknot, projected to the plane in an obvious way (figure 22). Summing over the two possible labelings of the diagram, we arrive at the result $-q^{1 / 2}-q^{-1 / 2}$.

For a slightly less trivial example, we consider the two projections of a single strand to the $x^{1}-x^{3}$ plane shown in figure 23 . In either (a) or (b), the knot projections shown on the left or right can be deformed into one another, so one might expect them to be equivalent. But in the present context, this is actually not the case. The twist of (a) relative to (b) introduces a factor of $-q^{3 / 4}$, which can be evaluated by making use of the weights of the vertex model. It is instructive to actually do this; the same factor $-q^{3 / 4}$ arises whether the magnetic charge of the strand is +1 or -1 , but the details of the computation are quite different in the two cases.

Since the factor $-q^{3 / 4}$ does not depend on the magnetic charge carried by a given strand, it is universal: adding a twist of the type shown in the figure to any strand in an arbitrary knot or link multiplies the associated invariant by $-q^{3 / 4}$. A similar twist of the opposite handedness multiplies the invariant by $-q^{-3 / 4}$, for similar reasons. This factor means that the invariant associated to a knot (or link) by the quantum field theory depends on a choice of "framing". A framed knot is a knot that is slightly thickened into a ribbon. One keeps track of how the ribbon is twisted and (in the present context) adding a twist multiplies the invariant by a factor of $-q^{3 / 4}$. 
(a)

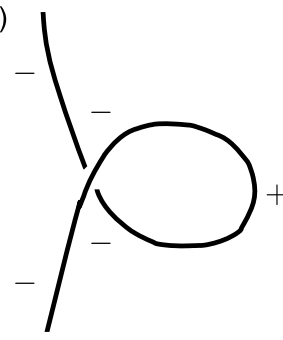

(b)

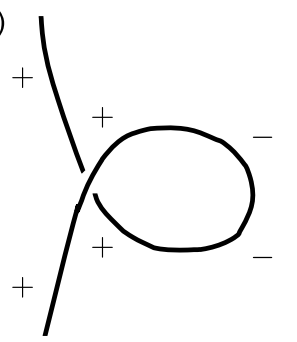

$=-q^{3 / 4}$
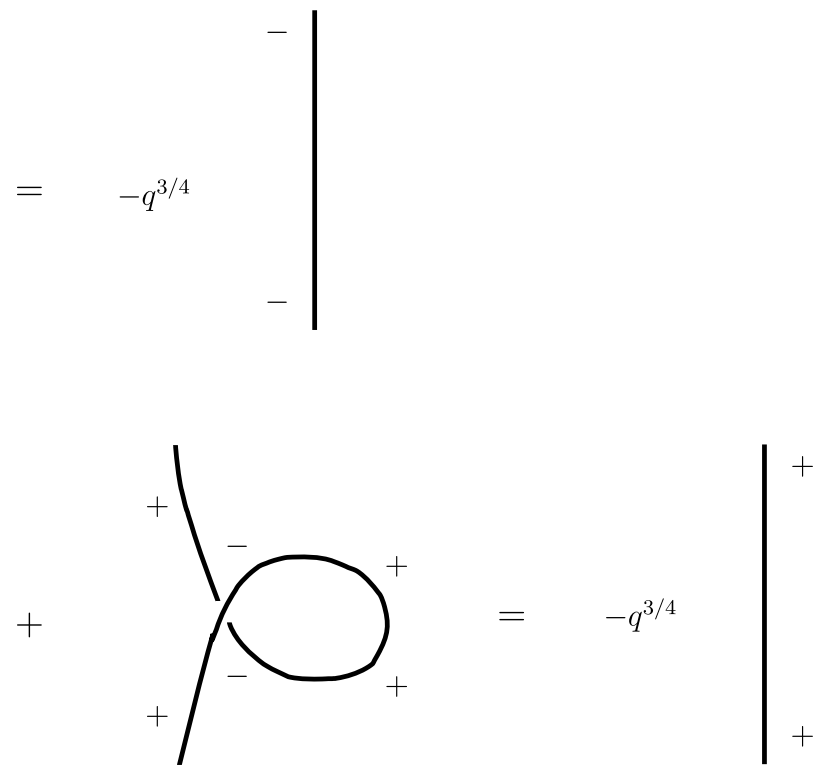

Figure 23: Use of the vertex model to compare two different projections of a single strand to the $x^{1}-x^{3}$ plane - with a twist (left) or no twist (right). For either sign of the charge carried by the strand, the twist introduces a factor of $-q^{3 / 4}$. The computation is quite different for the two possible values of the charge carried by the strand; for charge -1 , as shown in (a), the vertex model sum has only one nonzero contribution, corresponding to the indicated labeling, but for charge +1 , there are two possible contributions, shown in (b); they add to the same result. In (b), the second contribution involves a charge exchange process in which upper and lower strands exchange charge where they cross. A similar twist with undercrossing instead of overcrossing (or with the whole picture replaced by a mirror image) leads instead to a factor of $-q^{-3 / 4}$, as the reader can verify.

A knot that is presented with a projection to a plane comes with a natural framing, given by a slight thickening in the vertical direction, normal to the plane. A little thought (or experimentation with a strip of paper) shows that although the pictures on the left and right of figure 23(a) or (b) are topologically equivalent if one ignores the framings, they do differ by one unit of framing.

In the context of three-dimensional knot invariants that are associated to two-dimensional conformal field theory, conformal primary fields in two dimensions are associated to line operators in three dimensions. If a conformal primary has dimension $h$, then in a unit change in framing, the corresponding line operator is multiplied by $\exp (2 \pi \mathrm{i} h)$. The factor $-q^{3 / 4}$ is 
indeed $\exp (2 \pi \mathrm{i} h)$, where the conformal dimension of the degenerate Virasoro primary field $V_{-k / 2 b}$ is

$$
h_{V}(k)=-\frac{k}{2}-\frac{k(k+2)}{4 b^{2}},
$$

and in addition $q=\exp \left(-2 \pi \mathrm{i} / b^{2}\right)$, and the vertex weights that we have described are for the minimum charge case $k=1$.

At this stage, an important detail arises. The vertex weights that we have described are appropriate for a certain natural normalization of the Jones polynomial, which has been used in the literature, for instance in [49]. However, a slightly different normalization arises in $S U(2)$ Chern-Simons theory. In Chern-Simons theory, the expectation value of an unknot labeled by the two-dimensional representation of $S U(2)$ is $q^{1 / 2}+q^{-1 / 2}$, which differs in sign from what we deduced in figure 22 using the vertex weights. (The sign is easily checked in Chern-Simons theory. Since $q=\exp \left(2 \pi \mathrm{i} /\left(\boldsymbol{k}^{\vee}+2\right)\right)$ in $S U(2)$ Chern-Simons theory, where $\boldsymbol{k}^{\vee}$ is the level, the weak coupling limit $\boldsymbol{k}^{\vee} \rightarrow \infty$ corresponds to $q=1$; for $q=1$, the expectation value of a Wilson loop in any representation is simply the dimension of the representation, or +2 for the two-dimensional representation.) Similarly, the dimension of a primary field related to a representation of $S U(2)$ of spin $j=k / 2$ is

$$
h_{\mathrm{CS}}(k)=\frac{k(k+2)}{4\left(\boldsymbol{k}^{\vee}+2\right)},
$$

so that the factor acquired in a unit change of framing is $\exp \left(2 \pi \mathrm{i} h_{\mathrm{CS}}\right)=q^{3 / 4}$, without the minus sign found in figure 23. Clearly the discrepancy in sign reflects the fact that $h_{V}(k)-h_{\mathrm{CS}}(k)=-k / 2$, independent of $b$ and $\boldsymbol{k}^{\vee}$.

The comparison with Chern-Simons theory is not necessarily a problem for the present paper, in which we have simply started with the fourdimensional gauge theory equations (1.1). However, one would like to know the best interpretation of these signs in the context of the duality presented in [14] between Wilson operators of Chern-Simons theory and singular monopoles at the boundary. We believe that the interpretation may be that the dual of a Wilson operator of spin $k / 2$ in Chern-Simons theory is actually a boundary 't Hooft operator that carries angular momentum $k / 2$ and is fermionic when $k$ is odd. (For 't Hooft operators defined on the boundary of a four-manifold, the relevant rotation group is $S O(2)$ or rather its double cover $\operatorname{Spin}(2)$; this group is abelian and has one-dimensional representations, labeled by the angular momentum $k / 2$.) The fermi statistics for odd $k$ would give a minus sign for every crossing (relative to what is 
presented in figure 20) and a minus sign for every closed loop; including these signs brings the results obtained by braiding of Virasoro degenerate fields in agreement with the results obtained by braiding in Chern-Simons theory.

Finally, it is instructive to compare the computation in figure 23 to an equivalent computation if the gauge group were simply $G=U(1)$ instead of $S O(3)$. There would be two differences. First, the factors in figure 21 associated to creation and annihilation of a pair of strands would simply be 1. (Those factors come entirely from integrating out massive degrees of freedom of the $S O(3)$ theory, in reducing to an effective abelian description at low energies.) Second, charge exchange processes are absent for $G=$ $U(1)$ (as there are no smooth monopoles), so the second contribution in figure 23(b) would be absent. A look back to figure 20 shows that for $G=U(1)$, evaluation of either figure $23(\mathrm{a})$ or (b) gives a simple factor of $q^{1 / 4}$, instead of $-q^{3 / 4}$. Two comments are in order:

- The minus sign of figure 23 is absent for $G=U(1)$ (and similarly the minus sign in figure 22 is absent).

- In Chern-Simons theory, the power of $q$ is the quadratic Casimir invariant of the relevant representation of $G^{\vee}$. The quadratic Casimir of a representation of highest weight $j$ is $j^{2}$ for $G^{\vee}=U(1)$ and $j(j+1)$ for $G^{\vee}=S U(2)$. For $j=1 / 2$, this gives $q^{1 / 4}$ or $q^{3 / 4}$ in the abelian and nonabelian cases, respectively.

\subsubsection{Gradient flow and strings}

We can give a more concrete physical interpretation to the gradient flows which occur when strands of appropriate charge cross. Propagation of magnetic charge from one strand to another can be described by motion of a magnetic monopole between the two strands. If we rotate the coordinates and think of the $x^{2}$ direction as "time", we should be able to see the relevant object as a time-independent solution in the presence of only real symmetry breaking.

In fact, we have already described precisely the necessary solution: it is associated to the bare Miura oper with a single Bethe root and no singular monopole that was described in equation (3.31). So we visualize the charge exchange process as propagation of an object in the $x^{2}$ direction, as in figure 24. This picture is oversimplified, as it ignores the existence of a fourth dimension, normal to the boundary $\mathbb{R}^{3}$ that contains the knots. An alternative picture showing the role of the $y$ direction is given in figure 25 . When propagating in the $x^{2}$ direction, the soliton settles at a value of $y$ that is given by the solution for the bare Miura oper. (How to compute this value 


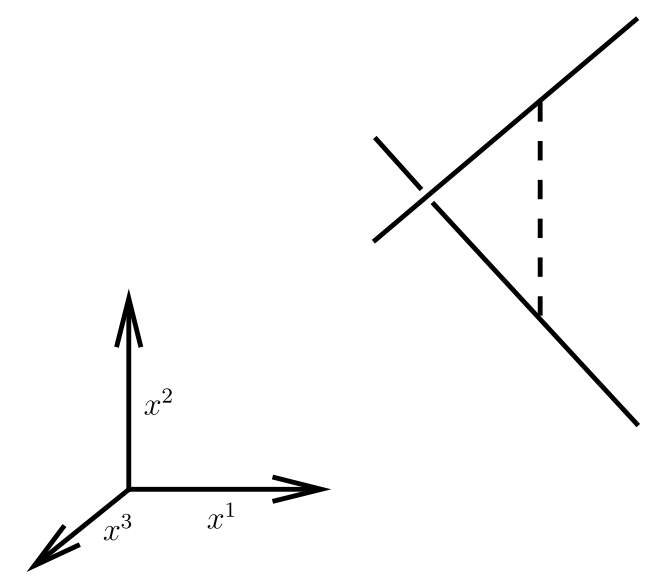

Figure 24: To make the charge exchange process more visible, we have exchanged the coordinate axes relative to figure 18. The $x^{2}$ axis now runs vertically while $x^{1}$ runs horizontally. Charge exchange occurs at values of $x^{1}$ at which two strands differ only in the value of $x^{2}$, so with the coordinate axes aligned as in this picture, the charge exchange involves a flow of charge in the vertical direction, represented by the dotted line. We take this to represent the propagation of a BPS soliton. The soliton propagates along the axis of symmetry breaking, so it is described by a solution with real symmetry breaking only - in fact, by the bare Miura oper of equation (3.31) with no 't Hooft operator and a single Bethe root.

is explained most fully in Section 7.) Of course, this description is only good if the soliton propagates far enough in $x^{2}$ that it has "time" to reach the equilibrium value of $y$. So it is only good if the strands are sufficiently far separated, or the symmetry breaking is strong enough.

We have gained an intuitive picture of the solutions of the fourdimensional BPS equations at strong symmetry breaking: they describe smooth monopole configurations stretched between the singular monopole strands. The contribution of each configuration to the knot invariant is then the product of two types of factor. One type arises from the map from the microscopic nonabelian theory to the abelian theory, while the other is computed in the abelian theory. Factors of the first type appear where pairs of strands are created or annihilated and where a smooth monopole is emitted or absorbed by a boundary singular monopole. In the abelian description, the smooth monopoles also look like singular monopoles, of charge 2, but not attached to the boundary. The whole configuration looks like a web of monopole strands, to which the abelian theory associates an overall power of $q$. 


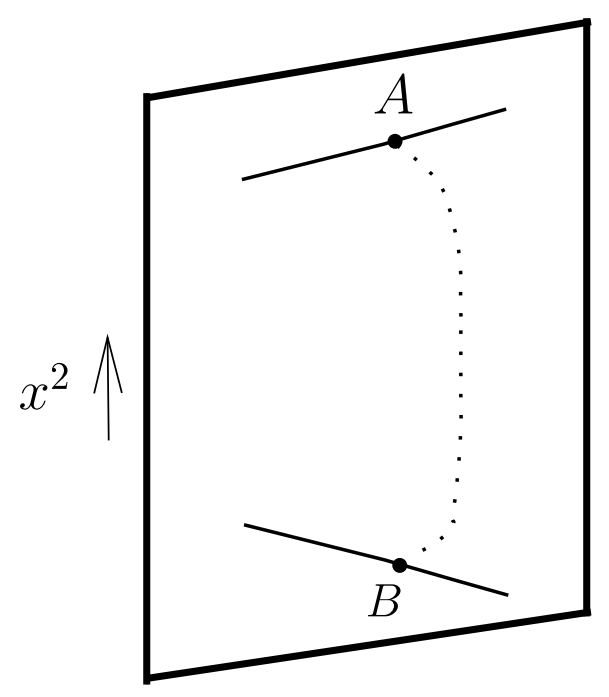

Figure 25: An alternative view of the charge exchange process that was pictured in figure 24, to show the role of the $y$ direction (here depicted as the direction normal to the plane that contains the two strands). A soliton propagating between two boundary points $A$ and $B$ that are separated by a long distance in the $x^{2}$ direction will bend away from the boundary, to reach a value of $y$ corresponding to the solution for the bare Miura oper.

\subsubsection{Analog in the dual Chern-Simons theory}

It is entertaining to carry this picture all the way back to three-dimensional Chern-Simons theory, before all the dualities which brought us to the fourdimensional description studied in the present paper. A key step in relating the two pictures is $S$-duality, which luckily is very transparent at strong symmetry breaking, as it reduces to electric-magnetic duality in the abelian gauge theory. We get immediately a sum over configurations of massive $W$-bosons stretched between Wilson lines, and only carrying electric charge toward the positive $x^{2}$ direction.

This suggests to look for a gauge condition in nonabelian Chern-Simons theory which would have this effect. It is easy to identify it: one can pick a "partial" axial gauge fixing which reduces the $A_{2}$ component of the gauge field to the Cartan subalgebra. We write the nonabelian gauge field as $A=B \mathfrak{t}_{3}+W_{+} \mathfrak{t}_{+}+W_{-} \mathfrak{t}_{-}$, and impose the $A_{2}=0$ gauge condition for $W_{ \pm} ;$ it will not be necessary here to make a gauge choice for the diagonal gauge field $B$. The action for charged $W$-bosons then reduces to

$$
\frac{\boldsymbol{k}^{\vee}}{4 \pi} \int d^{3} x W_{+} D_{2} W_{-}, \quad D_{2}=\frac{\partial}{\partial x^{2}}+\left[B_{2}, \cdot\right] .
$$


The equation for the propagator is

$$
D_{2} G(x, y)=\frac{2 \pi}{\boldsymbol{k}^{\vee}} \delta^{3}(x-y)
$$

and has a solution

$$
\frac{2 \pi}{\boldsymbol{k}^{\vee}} \exp \left(\int_{x^{2}}^{y^{2}} B_{2}\right) \delta\left(x^{1}-y^{1}\right) \delta\left(x^{3}-y^{3}\right) \theta\left(x^{2}-y^{2}\right),
$$

which only describes propagation of charge toward the positive $x^{2}$ direction. It is pretty clear that such a partial gauge fixing will lead to a version of the vertex model, although it may be tricky to compute the precise vertex weights. The necessary computations are likely to be somewhat similar to those involved in studying Chern-Simons theory in an ordinary axial gauge - for example, see [52,53] — or in a certain almost axial gauge [54]. Somewhat analogous is the use of a complex version of axial gauge to derive the Knizhnik-Zamolodchikov equations [55].

\subsection{Up to six dimensions}

With an eye to future categorification, it is useful to lift this abelian picture all the way to six dimensions. After all, in the presence of strong symmetry breaking, the six-dimensional $(0,2)$ theory is not that mysterious. It is a theory of self-dual two-forms coupled to heavy dynamical BPS strings. The six-dimensional setup which leads to knot invariants involves the sixdimensional theory on the product $\mathbb{R} \times M_{3} \times D$, where $D$ is a copy of $\mathbb{R}^{2}$ with the geometry of a semi-infinite cigar (see equation (7.28)). The knot itself is represented by a knotted two-dimensional defect placed at the tip of the cigar. The $q$-grading comes from the conserved angular momentum derived from the rotational symmetry of $D$.

In the absence of symmetry breaking, the fact that the metric on $D$ is cigar-like rather than being the Euclidean metric is important in order to get a well-defined space of states for the defect. A defect placed in flat six-dimensional space would be strongly coupled to the bulk SCFT. On the other hand, in the presence of symmetry breaking the IR physics is free, and we can hope to recover the space of states from bound states of the dynamical heavy strings and the defect. The cigar geometry would then not play a significant role, and $D$ can be replaced by a flat $\mathbb{R}^{2}$.

Now, we will specialize to $M_{3}=\mathbb{R}^{3}$, and for brevity we will take the knot to be time-independent. The BPS condition for a time-independent 
dynamical string is very simple: it must be straight, and aligned with the symmetry breaking (which we will still take to be the $x^{2}$ direction). This is literally the six-dimensional lift of the condition satisfied by the smooth monopoles, and leads to the same vertex-model picture when projected on $x^{2}$. The defect strands have two possible ground states in the abelian lowenergy description, of opposite two-form charge. A junction with a dynamical string allow the two-form charge to jump.

The interesting question is to reproduce the weights of the vertex model from six-dimensional considerations. This includes both contributions from the abelian theory of self-dual two-forms sourced by the strings, and contributions from the worldvolume theory of the strings. We will not compute the former here, but we can give some insight on the latter.

As summarized in figure 20, the vertex model weight for a charge exchange process contributing to the Jones polynomial is

$$
q^{ \pm 1 / 4}-q^{\mp 3 / 4} .
$$

In the six-dimensional picture, the Jones polynomial is supposed to come from a sum over BPS states, weighted by $q^{P}(-1)^{F}$, where $P$ is the conserved charge that corresponds to rotation of $D$, while $F$, which one might loosely call fermion number, is a certain $R$-symmetry generator. We interpret the relative factor $-q^{ \pm 1}$ between the two terms in (6.32) to mean that a BPS string connecting two strands in a knot has two physical states, differing by 1 in both angular momentum (to account for the factor of $q^{ \pm 1}$ ) and fermion number (to account for the minus sign). To understand this, we can focus on the two crossing strands, and the string stretched between them. Crucially, a single strand is half-BPS, two nonparallel strands are quarter-BPS, but the configuration of two strands exchanging a BPS string breaks symmetry further down to eighth-BPS. So the string breaks two supercharges, and hence it must have two ground states, exchanged by the action of the broken supercharges. This immediately gives the desired difference in quantum numbers.

Hence, the space of approximate ground states for the system can be described as follows. Let $\mathcal{S}$ be the set of configurations of the vertex model (labelings of strands by + or - , with smooth strings attached at crossings where a label jumps). For every smooth string, introduce a two-dimensional Hilbert space with quantum numbers derived from the last paragraph. To each $s \in \mathcal{S}$, introduce a Hilbert space $\mathcal{H}_{s}$ that is defined as the tensor product of the two-dimensional factors associated to the smooth strings. Then an approximation $\mathcal{H}_{0}$ to the space of BPS states is $\mathcal{H}_{0}=\oplus_{s \in \mathcal{S}} \mathcal{H}_{s}$. The grading of $\mathcal{H}_{0}$ (by angular momentum and fermion number) is affected by the abelian 
field configurations sourced by the system of strings; for example, the selfdual abelian tensor fields can carry angular momentum.

In order to compute Khovanov homology, one needs to evaluate the differential acting in this space of approximate ground states, by searching for instanton configurations which interpolate between different states in the past and future. The BPS condition for a time-dependent BPS string is still rather transparent. The worldsheet should be holomorphic in complex coordinates $x^{0}+\mathrm{i} x^{2}$ and $x^{1}+\mathrm{i} x^{3}$. Notice that a time-independent string stretched along the $x^{2}$ direction (and thus parameterized by $x^{0}$ and $x^{2}$, with $x^{1}$ and $x^{3}$ fixed) indeed has a holomorphic worldvolume.

Thus we expect to be able to build the differential for Khovanov homology from the data of holomophic curves in $\mathbb{R} \times M_{3}$ which end on the knot. This avenue seems promising for future development.

\section{An effective superpotential for monopoles}

\subsection{Overview of results}

Starting in Section 2.4, we interpreted solutions of three-dimensional supersymmetric equations in terms of configurations containing smooth BPS monopoles. However, the considerations were purely qualitative. In this section, we will make the reasoning quantitative. We will construct an effective superpotential for smooth BPS monopoles on $\mathbb{R}^{2} \times \mathbb{R}_{+}$interacting with a Nahm pole and singular monopoles on the boundary. This effective superpotential will account in a direct way for all qualitative results from Sections 2 and 3 about what solutions to our equations do or do not exist for various values of the parameters. Also, by integrating out some massive fields, we will be able to recover the Yang-Yang function (3.52) that has been one of our main tools.

Let us first consider our underlying supersymmetic equations

$$
\begin{aligned}
\left(F-\phi \wedge \phi+t d_{A} \phi\right)^{+} & =0 \\
\left(F-\phi \wedge \phi-t^{-1} d_{A} \phi\right)^{-} & =0 \\
d_{A} \star \phi & =0
\end{aligned}
$$

on $\mathbb{R} \times \mathbb{R}^{3}$ (where the first factor is the "time" direction) and ask how the solutions of Bogomolny equations for smooth monopoles can be embedded as solutions of these equations. This is possible precisely if $t=1$ (or -1 ), 
the only nonzero component of $\phi$ is the time component, which here we will call $\phi_{t}$ (rather than $\phi_{1}$, as before), and we also set the time component of $A$ to zero. Then equations (7.1) reduce to the Bogomolny equations

$$
F=\star d_{A} \phi_{t} .
$$

For the Bogomolny equations to have smooth monopole solutions, the field $\phi_{t}$ must have an expectation value at infinity, which means that the real symmetry breaking parameter $\boldsymbol{a}_{1}$ of Section 2.4 must be nonzero. On the other hand, the complex symmetry breaking parameter $\boldsymbol{a}$ of Section 2.5 must vanish (or $\phi_{t}$ would not be the only nonzero component of $\phi$ ).

The basic solution of the Bogomolny equations on $\mathbb{R}^{3}$ is the one-monopole solution for $G=S O(3)$ or $S U(2)$. It has has magnetic charge $\boldsymbol{m}=2$ (in units in which the basic singular monopole has charge $\boldsymbol{m}=1$ ). The moduli space of the one-monopole solution is $\mathcal{P}=\mathbb{R}^{3} \times S^{1}$, where $\mathbb{R}^{3}$ measures the center of mass position of the monopole on the spatial manifold $\mathbb{R}^{3}$, and $S^{1}$ is parameterized by a collective coordinate $\vartheta$ for the $U(1)$ gauge symmetry that is unbroken at infinity. Of course, $\mathcal{P}$ is a hyper-Kahler manifold; this follows from the unbroken supersymmetry of the Bogomolny equations on $\mathbb{R}^{3}$. However, since we will be considering perturbations that break some of the supersymmetry, for our purposes it is more useful to merely look at $\mathcal{P}$ as a complex manifold in one of its complex structures. We decompose $\mathbb{R}^{3}$ as $\mathbb{R}^{2} \times \mathbb{R}$ (which we will eventually replace with $\mathbb{R}^{2} \times \mathbb{R}_{+}$). The motion of the smooth monopole along $\mathbb{R}^{2}$ is parameterized by a chiral superfield $W$. And the position $y$ of the smooth monopole in the $\mathbb{R}$ direction combines with the collective coordinate $\vartheta$ to a second chiral superfield

$$
Y=\boldsymbol{a}_{1} y+\mathrm{i} \vartheta .
$$

Now we want to construct an effective superpotential $\mathcal{W}(W, Y)$ that describes this situation. If $\boldsymbol{a}=\zeta=0$, there is a smooth monopole solution for every value of $W$ and $Y$, which means that every value of $W$ and $Y$ is a critical point of $\mathcal{W}$, so $\mathcal{W}$ must vanish (modulo an irrelevant constant). On the other hand, if either $\boldsymbol{a}$ or $\zeta$ is nonzero, then there is no supersymmetric monopole solution, meaning that $\mathcal{W}$ has no critical point. But turning on $\boldsymbol{a}$ and $\zeta$ preserves the symmetries of adding a constant to $W$ or $Y$. So $\mathcal{W}$ must be invariant modulo an additive constant under constant shifts of $W$ or $Y$; in other words, $\mathcal{W}$ must be a linear function of $W$ and $Y$. In fact, the form of $\mathcal{W}$ is

$$
\mathcal{W}=a W+\zeta Y .
$$


This follows from the following considerations. Invariance under rotations of $\mathbb{R}^{2}$ implies that $\boldsymbol{a}$ and $W$ can only appear as the product $\boldsymbol{a} W$. On the other hand, although at $\zeta=0$, the superpotential $\mathcal{W}$ is given microscopically by the single-valued function (2.6), as soon as $\zeta$ becomes nonzero it is given by the Chern-Simons function (3.6), which is only single-valued $\bmod 2 \pi \mathrm{i} \mathbb{Z}$. Since $Y$ is similarly single-valued $\bmod 2 \pi \mathrm{i} \mathbb{Z}$ (because of the angular nature of $\vartheta$ ), the effective superpotential (7.4) has a multivaluedness that just matches that of the microscopic description.

Now let us turn off $\boldsymbol{a}$ and $\zeta$ but replace $\mathbb{R}^{2} \times \mathbb{R}$ by $\mathbb{R}^{2} \times \mathbb{R}_{+}$, where $\mathbb{R}_{+}$is the half-line $y \geq 0$ and we assume the usual Nahm pole at $y=0$. We assume symmetry breaking for $y \rightarrow \infty$ with $\phi_{t} \rightarrow \operatorname{diag}\left(\boldsymbol{a}_{1},-\boldsymbol{a}_{1}\right)$ while of course $A$ and $($ at $\boldsymbol{a}=0$ ) $\vec{\phi}$ vanish for $y \rightarrow \infty$. In any solution, the fields approach these asymptotic values exponentially fast for $y \rightarrow \infty$. This reflects the fact that near $y=\infty$, the gauge symmetry is reduced from $S U(2)$ to $U(1)$ by the expectation value of $\phi_{1}$, and all charged fields have masses proportional to $\left|\boldsymbol{a}_{1}\right|$. In particular, in the absence of singular or smooth monopoles, the solution for the full system (7.1) is given by a solution of Nahm's equations (the relevant solution is described in [16]) in which the fields approach their vacuum values exponentially fast for $y \rightarrow \infty$.

Next, still with $\boldsymbol{a}=\zeta=0$, let us add a smooth monopole with positions $W, Y$. There is not an exact solution for the smooth monopole, because the Nahm pole forces charged components of $\vec{\phi}$ to have nonzero values that "repel" the monopole to $y=\infty$. However, these charged fields are exponentially small for large $y$, so a smooth monopole located at large $y$ is exponentially close to being a solution. This being so, a smooth monopole that is located at large $y$ must be governed by an effective superpotential. This superpotential must be a single-valued function of $Y$ that vanishes exponentially for $y \rightarrow \infty$. These conditions are satisfied by a linear combination of exponentials $\exp (-n Y), n=1,2,3 \ldots$ However, it will soon become clear that the expected qualitative picture emerges if and only if at $a=\zeta=0, \mathcal{W}$ is linear in $\exp (-Y)$ :

$$
\mathcal{W}=\Lambda \exp (-Y)
$$

for some constant $\Lambda$.

In principle, it should be possible to compute this result by evaluating the microscopic superpotential (2.6) for an approximate solution consisting of a smooth monopole at large $y$ in the presence of a Nahm pole. The exponentially small term should come from $W$ boson exchange between the boundary and the monopole. Instead of attempting such a computation, we will take a shortcut in this paper: we will consider a representation of the 
smooth monopole and the Nahm pole by a configuration of branes, and in that context the exponential superpotential (7.5) will emerge from a simple brane instanton.

Postponing that analysis to Section 7.3, let us discuss the implications of (7.5). First we assume that $\boldsymbol{a}=\zeta=0$, so that (7.5) is the full superpotential. We see at once that $\mathcal{W}$ has no critical point so there is no supersymmetric solution in the presence of the smooth monopole. This is in full accord with the analysis in Section 2.4. With real symmetry breaking only and $\zeta=0$, a supersymmetric solution is expected to be unique (this remains true even in the presence of singular monopoles at $y=0$, which we have not yet included in $\mathcal{W}$ ) and does not require smooth monopoles. Now let us see what happens when we turn on $\boldsymbol{a}$ and $\zeta$. We construct the full superpotential by simply adding the various terms that we have found so far:

$$
\mathcal{W}=\boldsymbol{a} W+\zeta Y+\Lambda \exp (-Y)
$$

The justification for including $\boldsymbol{a}$ and $\zeta$ in this way is the same as before ( $\Lambda$ may now depend on $\zeta$ ). Let us look for critical points. We see at once that there is no critical point unless $\boldsymbol{a}=0$ and $\zeta \neq 0$. If these conditions are satisfied, there is a one-parameter family of critical points, parameterized by $W$, with

$$
\exp (-Y)=\frac{\zeta}{\Lambda}
$$

(The uniqueness of the solution for $\exp (-Y)$ holds precisely because in (7.5) we took $\mathcal{W}$ to be linear in $\exp (-Y)$.) We found a similar result in Section 3.5.1 where we described in equation (3.31) a family of solutions that exist precisely if $\boldsymbol{a}=0$ and $\zeta \neq 0$. The solution in question corresponded to a Miura oper with only real symmetry breaking, a single Bethe root at an arbitrary point $w \in \mathbb{R}^{2}$, and no singular monopoles. We interpret the Bethe root $w$ as the value of the superfield $W$ - in other words, the position of the smooth monopole in $\mathbb{R}^{2}$ - while the position of the smooth monopole in the $\mathbb{R}_{+}$direction is determined in (7.7). The reason that this description makes sense is that for small $\zeta$, the smooth monopole is located at large $y$, where the effective superpotential $\mathcal{W}$ is valid.

The next step is to include singular monopoles. As in Section 2.2.1, in the presence of singular monopoles of charge $k_{a}$ located at positions $z_{a}, a=$ $1, \ldots, d$ in the complex plane, it is convenient to introduce the polynomial $K(z)=\prod_{a=1}^{d}\left(z-z_{a}\right)^{k_{a}}$. In Section 7.2, we will argue that the only effect of the singular monopoles is to multiply the exponential term by $K(W)$, so 
that the superpotential becomes

$$
\mathcal{W}=\boldsymbol{a} W+\zeta Y+\Lambda K(W) \exp (-Y)
$$

Now let us examine the implications of this formula. Suppose first that $\zeta=0$. Then the conditions for a critical point are

$$
K(W)=0=\boldsymbol{a}+\Lambda K^{\prime}(W) \exp (-Y)
$$

The first condition says that $W$ must equal one of the $z_{a}$. For $\boldsymbol{a}=0$ and all $k_{a}=1$, the second condition then has no solutions. If $k_{a}>1$ for some $a$, still with $\boldsymbol{a}=0$, the second condition is satisfied for arbitrary $Y$. All this is in keeping with what we found in Sections 2.3 and 2.4. Now suppose that $\boldsymbol{a} \neq 0$. If $k_{a}=1$, the second condition in (7.9) determines $Y$ uniquely, and if $\boldsymbol{a}$ is small, then $Y$ is large so that the analysis is valid. For $k_{a}>1$, the second condition in (7.9) cannot be satisfied. All these statements match what was found in Section 2.5 from a quite different point of view. ${ }^{12}$

Now let us consider the case that $\zeta \neq 0$. The most illuminating way to proceed is to integrate out the massive field $Y$ to generate an effective superpotential for $W$. For fixed $W$, the condition $\partial \mathcal{W} / \partial Y=0$ has the unique solution $\exp (-Y)=\zeta / \Lambda K(W)$. Setting $Y$ to this value and evaluating $\mathcal{W}$, we find (modulo an irrelevant constant)

$$
\mathcal{W}=\boldsymbol{a} W+\zeta \log K(W)=\boldsymbol{a} W+\zeta \sum_{a} k_{a} \log \left(W-z_{a}\right)
$$

But this is the Yang-Yang function (3.52) for the case of a single Bethe root $W=w$, modulo terms that depend only on the $z_{a}$ and not on $W$; the present derivation is not sensitive to those terms.

At this point, the reader hopefully would like to see a similar derivation leading to the general Yang-Yang function with any number of Bethe roots. For a general case with $q$ smooth monopoles, we describe the positions of the $i$ th smooth monopole by chiral superfields $W_{i}, Y_{i}, i=1, \ldots, q$. The definition of these fields is somewhat subtle and is discussed in Section 7.2. The expectation values of the $W_{i}$ will turn out to be the Bethe roots $w_{i}$ of

\footnotetext{
${ }^{12}$ In Section 2.5, we found, for general $k_{a}$ and $\boldsymbol{a} \neq 0$, a solution with no smooth monopoles at $W=z_{a}$ and a solution with $k_{a}$ of them. (These correspond to the two ways of solving $P Q=\left(z-z_{a}\right)^{k_{a}}$ such that $P$ and $Q$ have no common zero at $z=z_{a}$.) Since (7.8) is the superpotential for just one smooth monopole, it describes a solution with $k_{a}$ smooth monopoles only if $k_{a}=1$. The general analysis for arbitrary $k_{a}$ can be made and matched to Section 2.5 using the superpotential (7.11) for an arbitrary number of smooth monopoles.
} 
Section 3.4. As in that discussion, it is convenient to introduce the polynomial $Q(z)=\prod_{i=1}^{q}\left(z-W_{i}\right)$. It turns out that the generalization of the superpotential (7.8) to an arbitrary number of smooth monopoles is

$$
\mathcal{W}=\boldsymbol{a} \sum_{i} W_{i}+\zeta \sum_{i} Y_{i}+\Lambda \sum_{i} \frac{K\left(W_{i}\right)}{Q^{\prime}\left(W_{i}\right)} \exp \left(-Y_{i}\right)
$$

To recover the qualitative results of Section 2 , we first set $\zeta=0, \boldsymbol{a} \neq 0$. To find a critical point, the $W_{i}$ must each equal zeroes $z_{a}$ of $K$. Assuming that the charges $k_{a}$ are all 1 , no more than one of the $W_{i}$ may equal the same $z_{a}$ (otherwise a zero of $Q^{\prime}\left(W_{i}\right)$ cancels a zero of $K\left(W_{i}\right)$ and the condition $\partial \mathcal{W} / \partial Y_{i}=0$ is not obeyed). Summing over all values of $q$, there are a total of $2^{d}$ solutions - each $z_{a}$ may or may not be equal to one of the $W_{i}$. If we take $\boldsymbol{a} \rightarrow 0$, then all but one of these solutions (the one with no smooth monopoles at all) disappear, with $Y_{i} \sim \log (1 / \boldsymbol{a})$.

For $\zeta \neq 0$, just as in the derivation of (7.10), it is convenient to integrate out the massive fields $Y_{i}$. Modulo terms that do not depend on the $W_{i}$, the superpotential that we arrive at is precisely the Yang-Yang function:

$$
\begin{aligned}
\mathcal{W} & =\sum_{i} \boldsymbol{a} W_{i}+\zeta \sum_{i} \log \left(K\left(W_{i}\right) / Q^{\prime}\left(W_{i}\right)\right) \\
& =\sum_{i} \boldsymbol{a} W_{i}+\zeta \sum_{i, a} \log \left(W_{i}-z_{a}\right)-2 \zeta \sum_{i<j} \log \left(W_{i}-W_{j}\right) .
\end{aligned}
$$

The attentive reader may notice one gap in what we have said. In the case $\zeta=0$, we have not analyzed the problem with $k_{a}>1$ for some $a$. To do this, it is important to consider the case that $W_{i}=W_{j}=z_{a}$ for some $i, j$, but the coordinates that we have used to describe the monopole moduli space are actually not adequate when $W_{i}=W_{j}$. We explain a better description momentarily.

\subsection{Coordinates for monopoles}

The moduli space of several smooth BPS monopoles on $\mathbb{R}^{3}$ is the subject of a rich mathematical theory [56]. From this theory we only need a small part: when the monopoles are widely separated in space (or equivalently when the symmetry breaking is strong), an effective abelian description of the moduli space is possible. In this description, the monopoles are regarded as "point" Dirac monopoles that interact with each other via the abelian gauge multiplet. Each monopole has a position in $\mathbb{R}^{3}$ and an angular coordinate $\vartheta$ that is a collective coordinate for charge rotations. As in Section 7.1, once 
we pick a particular complex structure on the moduli space, the position and angular coordinate of each monopole combine to a pair of chiral superfields $W, \mathcal{Y}$. However, there is a subtlety in the definition of $\mathcal{Y}$, which is the reason that we have changed our notation from Section 7.1.

The angular part of the coordinate $\mathcal{Y}$ parameterizes the freedom to do a $U(1)$ gauge transformation on the smooth monopole solution before "gluing" it to the abelian solution. Hence $\mathrm{e}^{\mathcal{Y}}$ is an holomorphic section of the $U(1)$ gauge bundle over the $W$-plane. This is the reason that in the presence of boundary singular monopoles, the exponential superpotential $\exp (-\mathcal{Y})$ for a single smooth monopole needs a prefactor $K(W)$ : the superpotential should be a function, but the singular monopoles make $\mathrm{e}^{-\mathcal{Y}}$ into the section of a bundle $\otimes_{a} \mathcal{O}\left(z_{a}\right)^{-k_{a}}$, where the exponents are the charges of the singular monopoles in the abelian effective field theory. So we compensate for this by multiplying by $K(W)$. A more intuitive explanation is that the superpotential encodes the interaction of the BPS monopole with the off-diagonal part of the complex Higgs field $\varphi$, which has a zero of order $k_{a}$ at $z_{a}$. So the superpotential acquires a factor $\left(z-z_{a}\right)^{k_{a}}$.

On the other hand, in the abelian theory, the BPS monopole behaves like a singular monopole. At any given value of $y$, one can restrict the $U(1)$ gauge bundle of the low-energy description to the $W$ plane. As one increases $y$ so that one passes the location of a BPS monopole, the $U(1)$ gauge bundle on the $W$ plane jumps. In other words, a second BPS monopole to the right of the first will feel the presence of a singular monopole of charge +2 at the location $W_{1}$ of the first monopole. Hence we expect a superpotential

$$
\mathcal{W}=K\left(W_{1}\right) \exp \left(-\mathcal{Y}_{1}\right)+\frac{K\left(W_{2}\right)}{\left(W_{2}-W_{1}\right)^{2}} \exp \left(-\mathcal{Y}_{2}\right)
$$

and similarly for several monopoles with increasing values of the real parts of $\mathcal{Y}_{i}$ :

$$
\mathcal{W}=\sum_{i} \frac{K\left(W_{i}\right)}{\prod_{j<i}\left(W_{i}-W_{j}\right)^{2}} \exp \left(-\mathcal{Y}_{i}\right)
$$

This superpotential is equivalent to the relevant part of (7.11) as long as the $W_{i}$ are distinct. The two are related by the change of variables

$$
\exp \left(-\mathcal{Y}_{i}\right)=\frac{\prod_{j<i}\left(W_{i}-W_{j}\right)}{\prod_{j>i}\left(W_{i}-W_{j}\right)} \exp \left(-Y_{i}\right)
$$

This re-definition does not affect the part of the superpotential linear in $\boldsymbol{a}$ and $\zeta$, since $\sum_{i} \mathcal{Y}_{i}=\sum_{i} Y_{i}$. However, it is the $\mathcal{Y}_{i}$, not the $Y_{i}$ whose real 
parts are the actual positions of the monopoles in the $y$ direction; moreover, the difference between the $\mathcal{Y}_{i}$ and the $Y_{i}$ is divergent when two or more $W_{i}$ coincide. The superpotential expressed in terms of the $\mathcal{Y}_{i}$ reproduces correctly the counting of solutions at $\zeta=0$ for arbitrary values of the $k_{a}$. For $\boldsymbol{a}=0$, if $K(z)=z^{k_{a}}$ the superpotential is

$$
\mathcal{W}=\sum_{i=1}^{q} \frac{W_{i}^{k_{a}}}{\prod_{j<i}\left(W_{i}-W_{j}\right)^{2}} \exp \left(-\mathcal{Y}_{i}\right)
$$

This function is extremized for arbitrary $\mathcal{Y}_{i}$ if the $W_{i}$ are all zero and the number $q$ of smooth monopoles is no greater than $k / 2$, because the prefactors have a zero of order at least 2 when the $W_{i}$ are all zero. This reproduces what we found in Section 2.3. If we do the same computation in terms of the $Y_{i}$, we would seem to get solutions even when the number of monopoles at the origin is greater then $k_{a} / 2$, but the $Y_{i}$ are not good variables when the $W_{i}$ coincide. We will leave the case $\boldsymbol{a} \neq 0, \zeta=0$ to the reader. For $\zeta \neq 0$, the difference between the $Y_{i}$ and the $\mathcal{Y}_{i}$ is not important.

It is interesting to match the coordinates in the low-energy description to the exact nonabelian description of the monopole moduli space. The exact monopole moduli space is parametrized [57] by a scattering matrix for the operator $\mathcal{D}_{y}=D_{y}+\mathrm{i}\left[\phi_{t}, \cdot\right]$. The scattering matrix takes the form

$$
S=\left(\begin{array}{ll}
Q(z) & P(z) \\
\widetilde{P}(z) & R(z)
\end{array}\right) \quad Q R-P \widetilde{P}=1
$$

where $Q$ is a monic polynomial of order $q, P$ and $\widetilde{P}$ are polynomials of degree up to $q-1$, and $R$ is a polynomial of degree up to $q-2$. $P$ and $Q$ are necessarily coprime (this follows from the condition $Q R-P \widetilde{P}=1$ ), and both $\widetilde{P}$ and $R$ are uniquely determined by $Q$ and $P$. One can define coordinates $W_{i}, Y_{i}$ on the monopole moduli space by $Q\left(W_{i}\right)=0$ and $P\left(W_{i}\right)=\exp Y_{i}$. However, this definition does not work well if the $W_{i}$ are not distinct.

To find a parametrization that works better as long as the symmetry breaking is strong, we can proceed as follows. For a single monopole, the scattering matrix takes the form.

$$
S_{1}=\left(\begin{array}{cc}
\left(z-W_{1}\right) & \mathrm{e}^{\mathcal{Y}_{1}} \\
-\mathrm{e}^{-\mathcal{Y}_{1}} & 0
\end{array}\right) .
$$

This expression can be matched naturally to the fact that at low energies, the smooth monopole can be approximated as a singular Dirac monopole. 
It tells us that there are two solutions $\psi_{ \pm}$of the equation $\mathcal{D}_{y} \psi=0$ that behave as

$$
\begin{array}{ll}
\psi_{+} \sim \mathrm{e}^{a_{1} y / 2}\left(\begin{array}{l}
1 \\
0
\end{array}\right) & y \ll 0, \\
\psi_{+} \sim\left(z-W_{1}\right) \mathrm{e}^{a_{1} y / 2}\left(\begin{array}{l}
1 \\
0
\end{array}\right)+\mathrm{e}^{\mathcal{Y}_{1}-a_{1} y / 2}\left(\begin{array}{l}
0 \\
1
\end{array}\right) & y \gg 0, \\
\psi_{-} \sim \mathrm{e}^{-a_{1} y / 2}\left(\begin{array}{l}
0 \\
1
\end{array}\right) & y \gg 0, \\
\psi_{-} \sim\left(z-W_{1}\right) \mathrm{e}^{-a_{1} y / 2}\left(\begin{array}{l}
0 \\
1
\end{array}\right)+\mathrm{e}^{-\mathcal{Y}_{1}+a_{1} y / 2}\left(\begin{array}{l}
1 \\
0
\end{array}\right) & y \ll 0 .
\end{array}
$$

Here $\psi_{+}\left(\psi_{-}\right)$is the unique solution which is small for $y \ll 0(y \gg 0)$. A singular monopole solution in the abelian theory would have given the same exponential growth for $y \gg 0(y \ll 0)$, and the subexponential correction is due to the exponentially decreasing corrections for the smooth monopole solution.

For a configuration of many well-separated smooth monopoles, the scattering matrix is a product

$$
S=S_{q} S_{q-1} \cdots S_{1} .
$$

where $S_{a}$ is the scattering matrix due to the ath monopole and the monopoles are taken to be ordered in the $y$ direction. It is natural to parameterize the moduli space by

$$
S_{a}=\left(\begin{array}{cc}
\left(z-W_{a}\right) & \mathrm{e}^{\mathcal{Y}_{a}} \\
-\mathrm{e}^{-\mathcal{Y}_{a}} & 0
\end{array}\right) .
$$

This enables us to write $P$ and $Q$ in terms of the $W_{i}$ and $\mathcal{Y}_{i}$, and finally, using $P\left(W_{i}\right)=\exp \left(Y_{i}\right)$, to express the $Y_{i}$ in terms of the $W_{i}$ and the $\mathcal{Y}_{i}$.

\subsection{Realization via $M$-theory and branes}

Here, we will explain an $M$-theory approach to understanding the exponential superpotential (7.5). We begin with some preliminaries.

\subsection{1 $M$-theory preliminaries}

The six-dimensional $(0,2)$ model of type $A_{1}$ can be realized on a pair of parallel M5-branes. Thus, we begin on $\mathbb{R}^{11}$ with coordinates $x^{0}, \ldots, x^{10}$, 
and we consider two M5-branes parameterized by $x^{0}, \ldots, x^{5}$ and located at $x^{6}=\cdots=x^{10}=0$. This system preserves 16 global supersymmetries. Their generators can be understood as eleven-dimensional spinors $\varepsilon$ that obey

$$
\Gamma_{0} \Gamma_{1} \cdots \Gamma_{5} \varepsilon=\Gamma_{6} \Gamma_{7} \cdots \Gamma_{10} \varepsilon=\varepsilon
$$

Here the gamma matrices obey $\left\{\Gamma_{\mu}, \Gamma_{\nu}\right\}=2 g_{\mu \nu}$.

To simplify the picture, we introduce symmetry breaking, separating the two M5-branes in, say, the $x^{6}$ direction. So now we place one at $x^{6}=0$ and the other at $x^{6}=L$, for some $L$. At low energies, this system is described by a pair of abelian tensor multiplets, coupled to BPS strings. The strings arise from M2-branes stretched between the two M5-branes. The string tension is $T=T_{\mathrm{M} 2} L$, where $T_{\mathrm{M} 2}$ is the M2-brane tension.

We will consider a string whose world-volume is parametrized by $x^{0}$ and $x^{1}$, and that is located at specified values of $x^{2}, \ldots, x^{5}$. The string is of course represented by an M2-brane that stretches from $x^{6}=0$ to $x^{6}=L$, although this direction will just factor out of the following analysis.

The string described in the last paragraph preserves those supersymmetries whose generator obeys

$$
\Gamma_{0} \Gamma_{1} \Gamma_{6} \varepsilon=\varepsilon
$$

as well as (7.23). Altogether, there are eight unbroken supersymmetries, corresponding to $\mathcal{N}=4$ supersymmetry in the two-dimensional sense. There is an $S O(4)$ symmetry group rotating the $x^{2}, \ldots, x^{5}$ coordinates, and a second $S O(4)$ symmetry, which we will call $S O(4)_{R}$, that rotates $x^{7}, \ldots, x^{10}$.

However, we will soon modify the construction in a way that will break half of the supersymmetry and also reduce $S O(4) \times S O(4)_{R}$ to a maximal torus. So it will help to focus on the relevant symmetries to begin with. We consider the $\mathcal{N}=2$ subalgebra consisting of supersymmetries that (in addition to the conditions already given) are invariant under a combined rotation of (say) the $x^{4}-x^{5}$ plane together with an $R$-symmetry rotation of the $x^{9}-x^{10}$ plane:

$$
\left(\Gamma_{4} \Gamma_{5}+\Gamma_{9} \Gamma_{10}\right) \varepsilon=0 .
$$

Given (7.24), this is equivalent to

$$
\left(\Gamma_{2} \Gamma_{3}+\Gamma_{7} \Gamma_{8}\right) \varepsilon=0
$$


We note that equations (7.25) and (7.26) are exchanged if we exchange $W=x^{2}+\mathrm{i} x^{3}$ with $Z=x^{4}+\mathrm{i} x^{5}$, and similarly exchange $x^{7}, x^{8}$ with $x^{9}, x^{10}$. So in particular, our conditions on $\varepsilon$ are symmetrical between $W$ and $Z$.

The $\mathcal{N}=2$ supersymmetry algebra singled out by the above conditions has a $U(1) \times U(1)$ group of $R$-symmetries generated by $J=\Gamma_{7} \Gamma_{8}$ and $J^{\prime}=$ $\Gamma_{9} \Gamma_{10}$. We want to compare $J$ and $J^{\prime}$ to $R$-symmetry generators that we will call $J_{+}$and $J_{-}$that only act, respectively, on supersymmetry generators that have positive or negative two-dimensional chirality, in other words, that obey $\chi \varepsilon= \pm \varepsilon$, where $\chi=\Gamma_{0} \Gamma_{1}$ is the two-dimensional chirality. The conditions given above can be combined to give

$$
\chi \varepsilon=J J^{\prime} \varepsilon
$$

and this implies that (with a suitable choice of sign for $J_{+}$and $J_{-}$, and normalizing them so that they square to 1 on supersymmetry generators of the appropriate chirality) $J$ and $J^{\prime}$ can be expressed as $J=J_{+}+J_{-}$, $J^{\prime}=J_{+}-J_{-}$.

In particular, an exchange $J \leftrightarrow J^{\prime}$ amounts to $J_{ \pm} \rightarrow \pm J_{ \pm}$, an operation known as the mirror symmetry automorphism of the $\mathcal{N}=2$ algebra. The automorphism of the above-described $\mathcal{N}=2$ algebra that exchanges $W$ and $Z$ also exchanges $J$ and $J^{\prime}$, so it is a mirror symmetry. Hence, if we view $W$ as a chiral superfield in the two-dimensional worldsheet theory of the string, we must view $Z$ as a twisted chiral superfield, in the sense of [58].

\subsubsection{Reduction to gauge theory}

So far, we have half-BPS strings, but no gauge theory description of them.

To get a gauge theory description, we compactify one direction, say the $x^{5}$ direction, on a circle of radius $R$. $M$-theory on a circle reduces at long distances to Type IIA superstring theory. The M5-branes become D4-branes and the theory on the D4-branes is at long distances a $U(2)$ gauge theory, broken to $U(1) \times U(1)$ by the separation between the D4-branes. What is relevant to us is the $S U(2)$ subgroup, broken at low energies to $U(1)$. The string that was originally described via a stretched M2-brane is now represented by a D2-brane stretched between the two D4-branes.

This situation has been studied in [59]. The D2-brane stretched between two D4-branes carries magnetic charge and corresponds to a smooth BPS monopole in the low-energy $S U(2)$ gauge theory.

Before compactifying the $x^{5}$ direction, the low-energy theory along the string was described by the chiral superfield $W=x^{2}+\mathrm{i} x^{3}$ and the twisted 
chiral superfield $Z=x^{4}+\mathrm{i} x^{5}$. After the compactification, we can replace $Z$ by the single-valued field $\exp (Z / R)$, which is still a twisted chiral superfield. However, for matching to the theory of BPS monopoles, another variable is more useful. The moduli of the BPS monopole corresponding to the D2brane are the positions of the underlying M2-brane in $x^{2}, x^{3}$, and $x^{4}$, and the dual of its position in $x^{5}$. The duality in question is a $T$-duality in the two-dimensional effective field theory governing the string. This $T$-duality is a mirror symmetry in the two-dimensional sense. It replaces $x^{5} / R$ with a new angular coordinate $\vartheta$. So while $Z / R=x^{4} / R+\mathrm{i} x^{5} / R$ is a twisted chiral superfield, $Y=x^{4} / R+\mathrm{i} \vartheta$ is an ordinary chiral superfield, just like $W$. Of course, the single-valued chiral superfield is not $Y$ but $\mathrm{e}^{Y}$.

We conclude with two comments:

- The fact that the angular coordinate $\vartheta$ of the BPS monopole is $T$-dual to the angular position $x^{5}$ is part of the relation between M5-branes on a circle and D4-brane gauge theory. The symmetry that rotates $x^{5}$ becomes instanton number in the $(4+1)$-dimensional gauge theory, while the symmetry that rotates $\vartheta$ is electric charge.

- The single-valued field $\Omega=\exp (Z / R)$ can be understood as a map to $\mathbb{C}^{*}$; it can be neither zero nor infinity. In Section 7.3.3, we modify the problem to make it possible to have $\Omega=0$.

\subsubsection{Reducing on a half-space}

To get a nontrivial superpotential, we will have to break some of the translation symmetries of the problem. In fact, we are interested in gauge theory on a half-space, so we want to restrict $y=x^{4}$ to be non-negative.

The gauge theory problem studied in the present paper arises if $x^{4}$ and $x^{5}$ parameterize not $\mathbb{R} \times S^{1}$, as is the case in our presentation so far, but rather a copy of $\mathbb{R}^{2}$ with a cigar-like metric

$$
d s^{2}=d y^{2}+f(y)\left(d x^{5}\right)^{2} .
$$

Here $f(y) \sim y^{2} / R^{2}$ for $y \rightarrow 0$ and $f(y) \rightarrow 1$ for $y \rightarrow \infty$. In fact, this was the starting point in the derivation in [14].

As a complex manifold, $\mathbb{R}^{2}$ is the same as $\mathbb{C}$, so we can now parameterize the $x^{4}$ and $x^{5}$ directions by a $\mathbb{C}$-valued chiral superfield $\Omega$, which asymptotically at large $y$ (but only there) can be written

$$
\Omega=\exp (Z / R), \quad Z=x^{4}+\mathrm{i} x^{5}
$$


In contrast to the concluding remark of Section 7.3.2, $\Omega$ is now $\mathbb{C}$-valued rather than $\mathbb{C}^{*}$-valued, and in particular there is no problem in having $\Omega=0$.

We will make use of this shortly in generating a superpotential.

\subsubsection{The instanton}

We now want to describe an M2-brane instanton that will generate the superpotential that we are looking for.

The instanton is supposed to correct the physics of a string that is parameterized by a worldsheet coordinate $X=x^{0}+\mathrm{i} x^{1}$. The string is located at definite values of $W$ and $\Omega$, say $W=W_{0}, \Omega=\Omega_{0}$.

We can understand qualitatively what sort of instanton can generate a superpotential. We consider a two-dimensional model with $\mathcal{N}=2$ supersymmetry whose chiral ring is generated at the classical level by the chiral superfield $W$ and whose twisted chiral ring is generated by the twisted chiral superfield $\Omega$. The chiral ring is the ring of observables of a twisted $B$-model, and the superpotential that we want to generate will give a deformation of this chiral ring. A hypothetical superpotential must be generated by configurations that preserve the $B$-model supersymmetry. These are configurations in which the chiral superfield $W$ is constant, while the twisted chiral superfield $\Omega$ is holomorphic as a function of the worldsheet coordinate. (It is a familiar fact that $B$-model supersymmetry requires a chiral superfield to be constant. The fact that $B$-model supersymmetry allows a twisted chiral superfield to be holomorphic is mirror to the perhaps more familiar fact that $A$-model supersymmetry allows a chiral superfield to be holomorphic.)

The instanton that generates the superpotential is accordingly given by $W=W_{0}$ while $\Omega$ is a nontrivial but simple holomorphic function of $X$ :

$$
\frac{\Omega}{\Omega_{0}}=X-X_{0},
$$

(Note that this formula only makes sense because $\Omega$ is allowed to vanish.) Here $X_{0}$ is a constant, which we interpret as the instanton position; as always in instanton physics, to calculate physical amplitudes, it is necessary to integrate over the instanton moduli, which here mean $X_{0}$ as well as some fermionic moduli associated to the supercharges under which the instanton solution is not invariant.

The worldvolume of an M2-brane instanton is supposed to be a threemanifold. The three-manifold we want is just the product of the two- 
manifold $S$ that was defined in (7.30) with the one-manifold $0 \leq x^{6} \leq L$ (all at $x^{7}=\cdots=x^{10}=0$ ).

Since it is invariant under $B$-model supersymmetry, and has no moduli except what follows from translation invariance and supersymmetry (the parameter $\Omega_{0}$ corresponds roughly to a constant value that $\Omega$ would have in the absence of the instanton), this sort of instanton will generate a superpotential. To understand just what superpotential will be generated, we use the asymptotic formula (7.29) and look at the disturbance in the string that is generated by the instanton, at great distances. For large values of $y=x^{4}$, we can write

$$
\exp \left(\left(y+\mathrm{i} x^{5}\right) / R\right)=\Omega_{0}\left(X-X_{0}\right) .
$$

We see that as $X$ circles once around $X_{0}$ in the clockwise direction (at large values of $\left|X-X_{0}\right|$ so that the formula (7.31) is valid), $x^{5}$ increases by $2 \pi R$. To produce this effect, the operator inserted at $X=X_{0}$ must be a twist field. As the $T$-dual of $x^{5}$ is the angular variable $\vartheta$, a twist field is $\exp (-\mathrm{i} \vartheta)$, and this must be the $\vartheta$-dependence of a superpotential that captures the effects of the instanton. The holomorphic expression must therefore be $\mathcal{W}=\Lambda \exp (-Y)$, where $Y=y+\mathrm{i} \vartheta$, for some constant $\Lambda$. This is precisely the result claimed in (7.5).

For further confirmation, and also to check the sign in the exponent of $\mathcal{W}$, let us consider the behavior of the field $y$ at large distances, far from $X=X_{0}$. At long distances, the fluctuations in $y$ are described by a free-field path integral

$$
\int D y \exp \left(-\frac{1}{4 \pi R^{2}} \int d x^{0} d x^{1}|\nabla y|^{2}\right) .
$$

When the operator $\exp (-y / R)$ is inserted in such a path integral at a point $X=X_{0}$, the result is that at large distances, $y / R$ grows as $\left|\log \left(X-X_{0}\right)\right|$. But this is exactly what we see in (7.31).

A final comment is that if the worldvolume dimension of the string were bigger than 2, we would have considered the instanton as a fluctuation around a vacuum defined by a limiting value of $y$ (and all the other worldvolume fields) for $X \rightarrow \infty$, and we would have asked for the instanton to approach this limiting value at infinity. In two dimensions, because of the usual infrared divergences - which appear, for instance, in the logarithmic growth mentioned in the last paragraph — such a formulation is not valid. 


\section{Opers and branes}

The purpose of the present section is to place some of the ingredients that have appeared in the present paper in a wider context.

We continue to study $\mathcal{N}=4$ super Yang-Mills theory, with a twist that preserves half the supersymmetry, on the four-manifold $M_{4}=\mathbb{R} \times C \times \mathbb{R}_{+}$, and with the usual Nahm pole boundary condition at the finite end of $\mathbb{R}_{+}$. The novelty, compared to what has been said so far, is that we will view the problem from the point of view of compactification on $C$ from four to two dimensions. In general $[60,61]$, assuming for simplicity that $C$ has genus at least 2 (we relax this condition in Section 8.4), compactification on $C$ gives at low energies a two-dimensional sigma-model in which the target is $\mathcal{M}_{\mathrm{H}}$, the moduli space of solutions of Hitchin's equations [20]. The Nahm pole boundary condition must reduce at low energies to a brane in this sigmamodel, and this brane must be half-BPS because the Nahm pole boundary condition is half-BPS in four dimensions.

\subsection{Back to $t=1$}

We begin by analyzing the case $t=1$. For simplicity, we take $G$ to be $S U(2)$ or $S O(3)$. In Section 2.2, we found that at $t=1$, the Nahm pole boundary condition (in the absence of singular monopoles) describes a Higgs bundle $(E, \varphi) \rightarrow C$ that is endowed with a line sub-bundle $L \subset E$ that is nowhere $\varphi$-invariant. Viewing $E$ as a rank 2 complex bundle of trivial determinant, the inclusion $L \subset E$ is part of an exact sequence:

$$
0 \rightarrow L \rightarrow E \rightarrow L^{-1} \rightarrow 0 .
$$

Here, we use the fact that, as $E$ has trivial determinant, the quotient $E / L$ must be isomorphic to $L^{-1}$.

We view $\varphi$ as a holomorphic map $E \rightarrow E \otimes K$, where $K$ is the canonical bundle of $C$. We can restrict $\varphi$ to $L$, to get a holomorphic map $L \rightarrow E \otimes K$, and then using the projection $E \rightarrow L^{-1}$, we get a map $\varphi: L \rightarrow L^{-1} \otimes K$. The condition that $L$ is nowhere $\varphi$-invariant means precisely that the map $\varphi: L \rightarrow L^{-1} \otimes K$ is everywhere nonzero. In other words, this map is an isomorphism.

Tensoring with $L$, we learn that $L^{2}$ is isomorphic to $K$, so that $L$ is a square root $K^{1 / 2}$ of $K$. If $G=S U(2)$, a solution of the Nahm pole boundary condition involves a choice of $K^{1 / 2}$, while if $G=S O(3)$, since we really 
should be working with the adjoint bundle ad $(E)$ rather than $E$, the choice of $K^{1 / 2}$ does not matter. In what follows, we assume that either $G=S O(3)$ or we have picked a particular square root of $K$.

It is possible to make a nontrivial extension $0 \rightarrow K^{1 / 2} \rightarrow E \rightarrow K^{-1 / 2} \rightarrow 0$, and we will exploit this fact in Section 8.2. However, for Higgs bundles, we want $E$ to be a direct sum $K^{1 / 2} \oplus K^{-1 / 2}$, since in the case of a nontrivial extension, the Higgs fields that we are about to write would not exist. If we write $E$ in column form

$$
E=\left(\begin{array}{c}
K^{-1 / 2} \\
K^{1 / 2}
\end{array}\right)
$$

then up to an automorphism of $E$, a possible Higgs field $\varphi$ takes the form

$$
\varphi=\left(\begin{array}{ll}
0 & 1 \\
q & 0
\end{array}\right)
$$

where $q$ is a quadratic differential. To be more exact, we assume the upper right matrix element of $\varphi$ to be nonzero as otherwise $L$ would be $\varphi$-invariant (and the Higgs bundle $(E, \varphi)$ would be unstable, as explained in [20]). Given this, by a bundle automorphism $\operatorname{diag}\left(\lambda, \lambda^{-1}\right)$, we can take the upper right matrix element to be 1 , and by a lower triangular bundle automorphism, we can make the diagonal matrix elements of $\varphi$ vanish. Finally, for $E$ as in (8.2), the lower left matrix element of $\varphi$ is a quadratic differential (an element of $\left.H^{0}\left(C, K^{2}\right)\right)$, which we call $q$.

Let $\mathcal{T} \subset \mathcal{M}_{H}$ be the submanifold parametrizing the Higgs bundles $(E, \varphi)$ described in the last paragraph. At $t=1$, the brane in $\mathcal{M}_{H}$ defined by the Nahm pole is supported on $\mathcal{T}$. What sort of subvariety is $\mathcal{T}$ ? As in [20], let $I$ be the complex structure on $\mathcal{M}_{H}$ in which it parameterizes Higgs bundles, $J$ the complex structure in which $\mathcal{M}_{H}$ parameterizes flat bundles with connection $\mathcal{A}=A+\mathrm{i} \phi$, and $K=I J$. The Hitchin fibration is the map from $\mathcal{M}_{H}$ to the space of quadratic differentials that maps $(E, \varphi)$ to $\operatorname{Tr} \varphi^{2}$. This map is holomorphic in complex structure $I$. For the Higgs field in (8.2), we have $\operatorname{Tr} \varphi^{2}=2 q$, so there is a unique such $\varphi$ for every desired value of $\operatorname{Tr} \varphi^{2}$. Accordingly, $\mathcal{T}$ is a holomorphic section of the Hitchin fibration; in fact it is the holomorphic section constructed in [20]. Actually, $\mathcal{T}$ is complex Lagrangian from the point of view of complex structure $I$. That assertion means that the complex symplectic form

$$
\Omega_{I}=\frac{1}{4 \pi} \int_{C} d \bar{z} d z \operatorname{Tr} \delta A_{\bar{z}} \delta \phi_{z}
$$


vanishes when restricted to $\mathcal{T}$. This is the case since, as the holomorphic type of $E$ is fixed for all Higgs bundles that represent points in $\mathcal{T}, \delta A_{\bar{z}}$ is zero (up to a gauge transformation) when restricted to $\mathcal{T}$.

Since $\mathcal{T}$ is complex Lagrangian in complex structure $I$, we can identify as follows the supersymmetry of the half-BPS brane produced by the Nahm pole. This is a brane of type $(B, A, A)$, that is, it is a $B$-brane in complex structure $I$, but an $A$-brane from the point of view of $J$ or $K$.

\subsection{General $t$}

At general $t$, we are dealing with a flat bundle rather than a Higgs bundle. The Nahm pole still gives a line sub-bundle $L \subset E$, so we still have an exact sequence

$$
0 \rightarrow L \rightarrow E \rightarrow L^{-1} \rightarrow 0
$$

as in (8.1). The covariant derivative $\mathcal{D} / \mathcal{D} z$ now gives a holomorphic map $E \rightarrow E \otimes K$. We can still restrict this map to $L$ and project the image to $L^{-1} \otimes K$, to get a linear map $\mathcal{D} / \mathcal{D} z: L \rightarrow L^{-1} \otimes K$. The condition that $L$ is nowhere invariant under $\mathcal{D} / \mathcal{D} z$ implies, just as in Section 8.1, that this map is an isomorphism from $L$ to $L^{-1} \otimes K$, and again we conclude that $L=K^{1 / 2}$.

The difference from Section 8.1 is that now the bundle $E$ is not a direct sum $K^{1 / 2} \oplus K^{-1 / 2}$ but a nontrivial extension. Indeed, as we assume that the genus of $C$ exceeds 1 , a bundle that holomorphically is a direct sum $K^{1 / 2} \oplus K^{-1 / 2}$ would not admit a flat connection.

Nontrivial extensions of $K^{-1 / 2}$ by $K^{1 / 2}$ are all isomorphic; this is so because such an extension is determined by an element of $H^{1}(C, K) \cong \mathbb{C}$, and the choice of a nonzero element does not matter, up to a bundle automorphism.

The simplest example of a flat bundle that from a holomorphic point of view is the extension described in the last paragraph can be found by placing on $C$ a Kahler metric of scalar curvature $R=-1$. Let $\omega$ be the spin connection of such a metric and $e$ the vierbein. The flat connection is

$$
\mathcal{A}=\omega \boldsymbol{t}_{3}+e_{\bar{z}} \boldsymbol{t}_{-}+e_{z} \boldsymbol{t}_{+} .
$$

In differential geometry, since $\omega$ is the spin connection, the flat bundle $E$ is the spin bundle of $C$, or more precisely the direct sum $K^{1 / 2} \oplus K^{-1 / 2}$ of the two spin bundles of opposite chirality. But in this basis, the complex 
structure of $E$ is defined by the $(0,1)$ part of $\mathcal{A}$, which is $\mathcal{A}_{\bar{z}}=\omega_{\bar{z}} \boldsymbol{t}_{3}+e_{\bar{z}} \boldsymbol{t}_{-}$; this is lower triangular, but not diagonal, so $E$ is an extension rather than a direct sum.

Having found a single flat connection $\mathcal{A}$ on the bundle $E$, it is straightforward to find them all. We do not want to change $\mathcal{A}_{\bar{z}}$ (since the holomorphic structure of $E$ is supposed to be unchanged), but we can change $\mathcal{A}_{z}$ by $\mathcal{A}_{z} \rightarrow \mathcal{A}_{z}+\lambda_{z}$, where (to preserve flatness) $\lambda_{z}$ is annihilated by $\mathcal{D}_{\bar{z}}$. For $E$ as described in the last paragraph, the relevant choice is $\lambda_{z}=q \boldsymbol{t}_{-}$, where $q$ is a quadratic differential.

Mathematically, a flat bundle $E \rightarrow C$ that from a holomorphic point of view fits in a nonsplit exact sequence (8.5) is called an oper; see [22] for a detailed explanation. We have learned that, at general $t$, the brane defined by the Nahm pole boundary condition is supported on the variety of opers. Actually, we should be more precise, because the Nahm pole boundary condition depends in general on a parameter $\zeta$ that was introduced in Section 3.1 , and the complex connection $\mathcal{A}$ that obeys the oper condition is in general not $A+\mathrm{i} \phi$ but the more general connection $\mathcal{A}^{\zeta}$ defined in equation (3.3). We write $\mathcal{V}_{\zeta}$ for the subvariety of $\mathcal{M}_{\mathrm{H}}$ defined by requiring that $\mathcal{A}^{\zeta}$ obeys the oper condition.

When restricted to $\mathcal{V}_{\zeta}, \mathcal{A}_{\bar{z}}^{\zeta}$ is fixed, up to a gauge transformation, so the complex symplectic form

$$
\Omega_{I_{\zeta}}=\frac{1}{4 \pi} \int_{C} d \bar{z} d z \operatorname{Tr} \delta \mathcal{A}_{\bar{z}}^{\zeta} \delta \mathcal{A}_{z}^{\zeta}
$$

vanishes. Accordingly, the brane $\mathcal{V}_{\zeta}$ is a complex Lagrangian brane, just as in Section 8.1, but now in a rotated complex structure. In the context of the present paper, the rotated complex structure is $I_{\zeta}$, defined in Section 3.1. $\mathcal{V}_{\zeta}$ might be called a brane of type $(B, A, A)_{\zeta}$, being related to complex structure $I_{\zeta}$ as a $(B, A, A)$ brane is to complex structure $I$.

As long as $\zeta \neq 0, \infty$, the complex structures $I_{\zeta}$ are all equivalent. If we simply set $\zeta=i$, we get the usual variety of opers for complex structure $J$; alternatively, in the limit $\zeta \rightarrow 0, \mathcal{V}_{\zeta}$ reduces to the holomorphic section $\mathcal{V}$ of the Hitchin fibration, described in Section 8.1.

\section{$8.3 \quad S$-duality}

A particularly simple boundary condition in $\mathcal{N}=4$ super Yang-Mills theory is the Neumann boundary condition for gauge fields, extended to the whole supermultiplet in a half-BPS fashion. In terms of branes, this is the boundary 
"ATMP-16-3-A5-GAI" — 2013/2/1 — 19:36 — page 1062 — \#128

condition for a family of D3-branes ending on a single NS5-brane in the absence of a gauge theory $\theta$-angle.

Upon compactification on $C$ and reduction to two dimensions, this boundary condition gives a brane $\mathcal{B}_{\mathrm{NS} 5}$ on $\mathcal{M}_{\mathrm{H}}$ corresponding to a trivial flat line bundle over $\mathcal{M}_{\mathrm{H}}$. In other words, the support of the brane $\mathcal{B}_{\mathrm{NS} 5}$ is all of $\mathcal{M}_{\mathrm{H}}$, and its Chan-Paton connection is trivial. The brane $\mathcal{B}_{\mathrm{NS} 5}$ is of type $(B, B, B)$, meaning that it is a $B$-brane in every complex structure. This reflects the fact that the trivial bundle over $\mathcal{M}_{\mathrm{H}}$ is holomorphic in every complex structure.

Under $S$-duality or electric-magnetic duality, the D3-NS5 boundary condition is converted to a D3-D5 boundary condition, still with $\theta=0$. On the other hand, $S$-duality acts in the dimensionally reduced theory as $T$ duality on the fibers of the Hitchin fibration $[60,61]$. Hence the brane $\mathcal{B}_{\mathrm{NS} 5}$ must be mapped by $S$-duality to a brane $\mathcal{B}_{\mathrm{D} 5}$ supported on a section of the Hitchin fibration. Moreover, the $S$-dual of a brane of type $(B, B, B)$ is of type $(B, A, A)$ (this is shown in [15]). For a middle-dimensional brane to be an $A$-brane, its support must be a Lagrangian submanifold, and its Chan-Paton bundle must be flat. So the section of the Hitchin fibration on which $\mathcal{B}_{\mathrm{D} 5}$ is supported must be complex Lagrangian from the point of view of complex structure $I$. On the other hand, concretely the D3-D5 boundary condition, for the case of a single D5-brane, is described by the Nahm pole [19] at $t=1$. (This value of $t$ corresponds to unbroken supersymmetry of type $(B, A, A)$.) Our analysis above determines the section of the Hitchin fibration that corresponds to the Nahm pole; it corresponds to the family of Higgs bundles described in (8.2) and (8.3).

The D3-NS5 boundary condition can be deformed by turning on $\theta$ and more generally by turning on a $U(1)$ gauge field on the NS5-brane. These deformations, which are described in [19], preserve the half-BPS nature of the boundary condition but rotate the unbroken supersymmetry. A particular deformation in this family, described in Section 12 of [15], gives a brane - the canonical coisotropic brane $\mathcal{B}_{\mathrm{cc}}$ — that is important in the gauge theory approach to the geometric Langlands correspondence. $\mathcal{B}_{\mathrm{cc}}$ is a rank one brane supported on all of $\mathcal{M}_{\mathrm{H}}$, with a Chan-Paton bundle whose curvature is a linear combination of the Kahler forms of $\mathcal{M}_{\mathrm{H}}$. The precise combination depends on a parameter analogous to our $\zeta$. With a choice that is convenient for geometric Langlands, the curvature of the Chan-Paton bundle is a multiple of $\omega_{J}$ (the Kahler form for complex structure $J$ ) and then $\mathcal{B}_{\mathrm{cc}}$ is of type $(A, B, A)$.

The $S$-dual of the deformation of $\mathcal{B}_{\mathrm{NS} 5}$ that gives $\mathcal{B}_{\mathrm{cc}}$ is a deformation of $\mathcal{B}_{\mathrm{D} 5}$ that is obtained by rotating the Nahm pole in the space of fields $\vec{A}$ 
and $\vec{\phi}$. This is analyzed in [19], and the appropriate type of "rotation" was briefly described in Section 3.1. As we have seen, the rotated Nahm pole boundary condition leads to a brane $\mathcal{B}_{\text {oper }}$ that is supported on the variety of opers, that is on $\mathcal{V}_{\zeta}$ for some $\zeta$. If $\mathcal{B}_{\mathrm{cc}}$ is defined in the standard fashion as a brane of type $(A, B, A)$, then its $S$-dual must have the same supersymmetry. In that case, $\mathcal{B}_{\text {oper }}$ is supported on the ordinary variety of opers, with $\zeta=i$. (In the present paper, it is more natural for $\zeta$ to be real.)

That the $S$-dual of the brane $\mathcal{B}_{\text {cc }}$ is the brane $\mathcal{B}_{\text {oper }}$ supported on the variety of opers is important in mathematical treatments of the geometric Langlands correspondence. See for example [62] for an explanation of the role of opers in the geometric Langlands correspondence. The facts that we have just described give a gauge theory way to understand the $S$-duality between $\mathcal{B}_{\text {cc }}$ and $\mathcal{B}_{\text {oper }}$. It has been argued [63] that the $S$-duality between these two branes is important in understanding the AGT correspondence [64] as well as recent developments relating supersymmetric gauge theory and integrable systems [65]. The role of the $S$-duality between $\mathcal{B}_{\text {cc }}$ and $\mathcal{B}_{\text {oper }}$ is more explicit in [66].

\subsection{Monodromy defects}

The concept of a Higgs bundle can be generalized by allowing singularities at isolated points $p_{i} \in C$. For what follows, the case of interest will be a regular singularity. To introduce a regular singularity near a point $p$ in $C$, we pick a local complex coordinate $z$ near $C$ and then we introduce polar coordinates $r, \theta$ with $z=r \mathrm{e}^{\mathrm{i} \theta}$. We select elements $\alpha, \beta, \gamma$ in the Lie algebra $\mathfrak{t}$ of a maximal torus $T \subset G$, and look for solutions of Hitchin's equations with a singularity at $p$ of the form

$$
\begin{aligned}
& A=\alpha d \theta+\cdots, \\
& \phi=\beta \frac{d r}{r}-\gamma d \theta+\cdots,
\end{aligned}
$$

where the ellipses refer to additional terms that are less singular than $1 / r$. We call this sort of codimension two singularity a monodromy defect. The general theory of Hitchin's equations adapts well to this situation [67] and one can define a moduli space $\mathcal{M}_{\mathrm{H}}(C ; p, \alpha, \beta, \gamma)$ of solutions that is a hyperKahler manifold with properties rather similar to what one has in the absence of the monodromy defect. Everything we will say generalizes in an obvious way to the case of any number of monodromy defects. 
Once we introduce monodromy defects, the limitation of some of the above statements to the case that the genus of $C$ is at least 2 can be dropped. All above statements hold for $C$ of any genus in the presence of a sufficient number of monodromy defects (for $G=S U(2)$, the required number is 3 if $C$ has genus 0 , and is 1 if $C$ has genus 1 ).

In the context of $\mathcal{N}=4$ super Yang-Mills on $\Sigma \times C$, where $\Sigma$ is another two-manifold, one can consider a monodromy defect supported on $\Sigma \times p$, with $p \in C$. The singular solution (8.8) of Hitchin's equations embeds naturally as a solution of the four-dimensional equations (1.1). In the limit that $C$ is small compared to $\Sigma$, the $\mathcal{N}=4$ theory on $\Sigma \times C$ reduces to a sigma-model on $\Sigma$ with target $\mathcal{M}_{\mathrm{H}}(C ; p, \alpha, \beta, \gamma)$. In this description, there is an additional parameter $\eta$ that arises [68] as a theta-angle for the abelian subgroup of $G$ that is unbroken along $\Sigma \times p$. So quantum mechanically, a monodromy defect really has four parameters $\alpha, \beta, \gamma, \eta$. Under $S$-duality, a monodromy defect of the above-described type in $G$ gauge theory is mapped to a similar monodromy defect in $G^{\vee}$ gauge theory. The transformation of the parameters under $S$-duality is $(\alpha, \eta) \rightarrow(\eta,-\alpha)$ while $\beta$ and $\gamma$ are rescaled (for more detail see Section 2.4 of [68]).

If we drop the subleading terms represented by the ellipses in (8.8), we find that the monodromy of the complex flat connection $\mathcal{A}=A+\mathrm{i} \phi$ is $U=$ $\exp (-2 \pi(\alpha-\mathrm{i} \gamma))$. The subleading terms do not modify the monodromy as long as $U$ is regular - meaning that the subgroup of $G$ that commutes with $U$ has dimension equal to $r$, the rank of $G$. If $U$ is not regular, there is an important subtlety, explained in detail in [68], Section 3.3. For brevity, we will here consider only the case that $G=S U(2)$, so that the nonregular values of $U$ are only \pm 1 . If $U= \pm 1$, the monodromy $V$ of a connection of the form (8.8) is not necessarily conjugate to $U$; on the contrary, generically it is in the "unipotent" conjugacy class containing the element

$$
U^{\prime}= \pm\left(\begin{array}{ll}
1 & 1 \\
0 & 1
\end{array}\right)
$$

A general element $V$ of this conjugacy class is \pm 1 plus an arbitrary nilpotent matrix:

$$
V= \pm 1+\left(\begin{array}{cc}
x & y \\
z & -x
\end{array}\right), \quad x^{2}+y z=0
$$

The equation $x^{2}+y z=0$ describes an $\mathrm{A}_{1}$ singularity $\mathbb{C}^{2} / \mathbb{Z}_{2}$. The singular point is located at $x=y=z=0$ where the monodromy $V$ is precisely \pm 1 ; in other words, this is the case that the subleading terms in (8.8) do not correct the monodromy. In setting $\alpha$ and $\gamma$ to special values at which $U= \pm 1$, we 


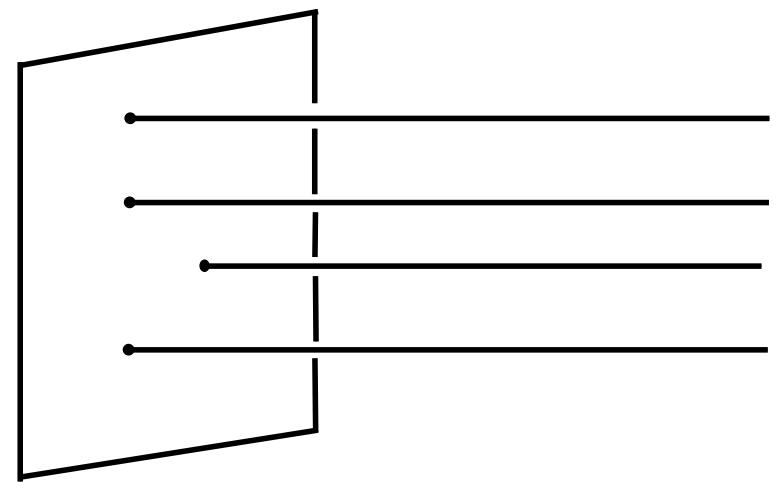

Figure 26: Monodromy defects in $C \times \mathbb{R}_{+}$. supported on $p_{i} \times \mathbb{R}_{+}$where the $p_{i}$ are points in $C$. $C$ is represented by the rectangle. We assume a Nahm pole boundary condition at $y=0$.

will assume that $\beta$ remains generic. In this case, even if $U= \pm 1$, the solution has a "symmetry breaking direction" built in, given by the singular term in the connection proportional to $\beta$. The effect of this is to blow up the $A_{1}$ singularity, replacing $\mathbb{C}^{2} / \mathbb{Z}_{2}$ with $T^{*} \mathbb{C P}^{1}$. This important fact is established in [67].

The precise meaning of this $T^{*} \mathbb{C P}^{1}$ is that if $U= \pm 1$ and $\beta=0$, then $\mathcal{M}_{\mathrm{H}}(C ; \alpha, \beta, \gamma)$ has a locus of $\mathrm{A}_{1}$ singularities, which parameterizes Higgs bundles for which the monodromy around $p$ is precisely \pm 1 . But if $\beta \neq 0$ with $U$ still equal to \pm 1 , then this singular locus is blown up, replacing the singularities by a family of $\mathbb{C P}^{1}$ 's.

The moduli space $\mathcal{M}_{\mathrm{H}}(C ; p, \alpha, \beta, \gamma)$ is invariant under shifting $\alpha$ by a cocharacter - for $G=S U(2)$, this means that it is invariant under $\alpha \rightarrow$ $\alpha+\operatorname{diag}(i,-i)$, which is a shift that can be induced by a gauge transformation that has a singularity at $p$. However, we will be interested in brane constructions that are not invariant under such shifts of $\alpha$, and for this reason, it will be best for our purposes not to view $\alpha$ as a periodic variable.

The first brane that we want to consider is the oper brane. It is defined as usual by the Nahm pole boundary condition. For this, we take $\Sigma$ to be $\mathbb{R} \times \mathbb{R}_{+}$, where $\mathbb{R}_{+}$is the usual half-line $y \geq 0$, and we impose the Nahm pole boundary condition at $y=0$. Suppressing the $\mathbb{R}$ or time direction, the picture on $C \times \mathbb{R}_{+}$is sketched in figure 26: there are monodromy defects supported on $p_{i} \times \mathbb{R}_{+}$, with respective parameters $\left(\alpha_{i}, \beta_{i}, \gamma_{i}, \eta_{i}\right)$, and a Nahm pole boundary condition at $y=0$. Of course, we need to explain what sort of singularity we want where the monodromy defect ends on a boundary with the Nahm pole. As usual, this kind of question is answered by finding 
a model solution with the desired singularity. For the present case, this has been done in Section 3.6 of [14], and in greater generality in [69].

Let us set $\alpha-\mathrm{i} \gamma=\lambda \operatorname{diag}(i,-i)$, for a complex parameter $\lambda$, and consider a Higgs bundle $E$ with a singularity of this type at, say, $z=0$. If $E$ (viewed in complex structure $I_{\zeta}$ ) is also an oper, then it can be described by the classical stress tensor

$$
t=-\frac{\lambda(\lambda+1)}{z^{2}}+\cdots
$$

where we have omitted less singular terms. This formula is just like (3.48), with $j_{a}=k_{a} / 2$ replaced by $\lambda$. Flat sections of $E$ correspond to holomorphic solutions of the differential equation

$$
\left(\frac{\partial^{2}}{\partial z^{2}}+t\right) \psi=0
$$

For generic $\lambda$, one can find two linearly independent solutions with $\psi_{1}=$ $z^{-\lambda}\left(1+\sum_{i=1}^{\infty} c_{i} z^{i}\right), \quad \psi_{2}=z^{\lambda+1}\left(1+\sum_{i=1}^{\infty} \widetilde{c}_{i} z^{i}\right)$. This means that, as expected, the monodromy is

$$
U=\operatorname{diag}(\exp (-2 \pi i \lambda), \exp (2 \pi \mathrm{i} \lambda)) .
$$

What happens if instead $\lambda=k / 2$ with $k \in \mathbb{Z}$ ? There is always a solution $\psi_{2}=z^{k / 2+1}\left(1+\sum_{i=1}^{\infty} \widetilde{c}_{i} z^{i}\right)$, but if we look for a solution with $\psi_{1}=$ $z^{-k / 2}\left(1+c_{1} z+\cdots\right)$, we find that generically when we carry this expansion to order $z^{k / 2+1}$, we need logarithmic terms of order $z^{k / 2+1} \log z+\cdots$. The logarithmic terms are simply a multiple of $(\log z) \psi_{2}$. Accordingly, the monodromy around $z=0$ is actually generically of the unipotent form

$$
\left(\begin{array}{l}
\psi_{1} \\
\psi_{2}
\end{array}\right) \rightarrow(-1)^{k}\left(\begin{array}{ll}
1 & s \\
0 & 1
\end{array}\right)\left(\begin{array}{l}
\psi_{1} \\
\psi_{2}
\end{array}\right)
$$

for some complex constant $s$.

So if we want the monodromy around $z=0$ to be trivial, we need to impose one condition on the subleading coefficients in the stress tensor (8.11), so as to get $s=0$. This means that having trivial monodromy around $z=0$ is a middle-dimensional condition. Indeed, without this condition, a monodromy defect for $G=S U(2)$ increases the complex dimension of $\mathcal{M}_{\mathrm{H}}$ by 2 , but the trivial monodromy condition fixes 1 of the 2 parameters.

As this point is important, we will dwell on it a bit. Generically, the monodromy around the defect is an element of $S L(2, \mathbb{C})$ (complex dimension 3 ) 
that obeys one constraint specifying its conjugacy class, leaving two complex parameters. For example, when $\alpha=\gamma=0$, the conjugacy class is twodimensional, as exhibited explicitly in (8.10). The condition of trivial monodromy (which is defined only when $U=\exp (-2 \pi(\alpha-\mathrm{i} \gamma)$ ) equals \pm 1 , and has no analog for other values) fixes one of the two parameters associated to the defect, so it leaves one parameter. One can think of this 1 parameter as the direction of symmetry breaking associated to the term $\beta d r / r$ in equation (8.8). The choice of a symmetry-breaking direction determines a point in a copy of $\mathbb{C P}^{1}$; this $\mathbb{C P}^{1}$ is the projectivization of the fiber of $E$ at the point $p \in C$ where the monodromy defect lives. A more detailed explanation of the origin of this $\mathbb{C P}^{1}$ is as follows. First of all, because of the equation $x^{2}+y z=0$, the unipotent conjugacy class described in equation (8.10) is explicitly isomorphic as a complex manifold to $\mathbb{C}^{2} / \mathbb{Z}_{2}$, with an $A_{1}$ singularity at $x=y=z=0$. The singularity is precisely the point at which the group element $V$ in (8.10) equals \pm 1 . In the context of the construction of $\mathcal{M}_{\mathrm{H}}$ as a hyper-Kahler manifold, the $\beta$ parameter is a Kahler parameter that blows up the $A_{1}$ singularity, replacing the conjugacy class $\mathbb{C}^{2} / \mathbb{Z}_{2}$ by its resolution, the Eguchi-Hansen manifold $T^{*} \mathbb{C P}^{1}$. In the blowup, the singular point at the origin is replaced by a copy of $\mathbb{C P}^{1}$. See [67] for the interpretation of $\beta$ as a blowup parameter, and [68] for a leisurely explanation of some of these matters.

We have essentially already run into the fact that in this situation, vanishing monodromy is a middle-dimensional condition. Let us specialize to the case that $C=\mathbb{C P}^{1}$ (we could similarly treat the case that $C=\mathbb{C}$ with an irregular singularity at infinity). We know from Section 3.4 that for a given set of singular points $z_{a}$ and charges $k_{a}, a=1, \ldots, d$, there are finitely many opers with monodromy-free singularities. The condition that a flat $G_{\mathbb{C}}$ bundle should be an oper is a middle-dimensional condition. To reduce to a finite set of opers with monodromy-free singularity, the condition of vanishing monodromy must also be middle-dimensional. (This assertion tacitly assumes that the two conditions intersect in a transverse fashion, which is in fact the case.)

In fact, dropping the oper condition, we can explicitly describe the moduli space of solutions of Hitchin's equations on $C$, with monodromy defects characterized by $\lambda_{a}=k_{a} / 2$, for which the complex connection $\mathcal{A}$ has trivial monodromy around those points. As $C$ is simply connected, a flat bundle on $C$ with no monodromy around the points $p_{a}$ is completely trivial as a flat bundle. The only possible moduli arise because the symmetry breaking associated to the parameters $\beta_{a}$ (which we assume to be all nonzero) generates a copy of $\mathbb{C P}^{1}$ at each singular point $p_{a}$. To get the moduli space, we must divide the product of these $\mathbb{C P}^{1}$ 's by the automorphism group of the trivial 
"ATMP-16-3-A5-GAI" — 2013/2/1 — 19:36 — page 1068 — \#134

flat bundle $E$; this is a copy of $S L(2, \mathbb{C})$. So finally the locus $\mathcal{U}$ of solutions of Hitchin's equations corresponding to flat bundles with trivial monodromy at each singular point is isomorphic to $\left(\mathbb{C P}^{1}\right)^{d} / S L(2, \mathbb{C})$. This is a complex submanifold of $\mathcal{M}_{\mathrm{H}}$ in complex structure $J$ (it is defined by a condition on the monodromies, which are holomorphic in that complex structure). Its dimension is $d-3$, which is one-half the dimension of $\mathcal{M}_{\mathrm{H}}$. In fact, $\mathcal{U}$ is complex Lagrangian from the point of view of complex structure $J$; this is true roughly because each $\mathbb{C P}^{1}$ is complex Lagrangian in $T^{*} \mathbb{C P}^{1}$. So the brane $\mathcal{B}_{\text {triv }}$ supported on $\mathcal{U}$ with trivial Chan-Paton bundle is a half-BPS brane of type $(A, B, A)$.

This gives us a new way to think about opers of trivial monodromy. They are intersection points of two Lagrangian submanifolds of type $(A, B, A)-$ one is the variety of opers and one parametrizes bundles with trivial monodromy. So the opers of trivial monodromy give a basis for the space of supersymmetric open strings stretching between the brane $\mathcal{B}_{\text {oper }}$ and the brane $\mathcal{B}_{\text {triv }}$. We call this the space of $\left(\mathcal{B}_{\text {oper }}, \mathcal{B}_{\text {triv }}\right)$ strings. Technically here we want the space of $\left(\mathcal{B}_{\text {oper }}, \mathcal{B}_{\text {triv }}\right)$ strings in the $B$-model of type $J$.

We can study this space of supersymmetric string states using $S$-duality, which converts the $B$-model of type $J$ to the $A$-model of type $\omega_{K}$. $S$-duality converts the brane $\mathcal{B}_{\text {oper }}$ to the canonical coisotropic brane $\mathcal{B}_{\text {cc }}$, as we learned in Section 8.3. It turns out that, as we describe shortly, $\mathcal{B}_{\text {triv }}$ is mapped to itself by $S$-duality (with the usual transformation of the monodromy defect parameters $\left.\left(\alpha_{a}, \beta_{a}, \gamma_{a}, \eta_{a}\right)\right)$. So the $S$-dual of the space of $\left(\mathcal{B}_{\text {oper }}, \mathcal{B}_{\text {triv }}\right)$ strings is the space of $\left(\mathcal{B}_{\text {cc }}, \mathcal{B}_{\text {triv }}\right)$ strings, now viewed in the $A$-model of type $\omega_{K}$. The key aspect of this problem is that although the support of $\mathcal{B}_{\text {triv }}$ is Lagrangian for $\omega_{K}$, it is actually symplectic for $\omega_{J}$ - indeed, the support of $\mathcal{B}_{\text {triv }}$ is a complex submanifold in complex structure $J$, and accordingly has $\omega_{J}$ as a Kahler form. This being the case, the problem of describing the space of $\left(\mathcal{B}_{\text {cc }}, \mathcal{B}_{\text {triv }}\right)$ strings is governed by the analysis of quantization and branes in [70]. The space of $\left(\mathcal{B}_{\mathrm{cc}}, \mathcal{B}_{\text {triv }}\right)$ strings is obtained by quantizing the support $\mathcal{U}$ of $\mathcal{B}_{\text {triv }}$; here $\mathcal{U}$ is viewed as a symplectic manifold with symplectic structure $\omega_{J}$.

\subsection{A selfdual brane}

There is a simple gauge theory explanation of why $\mathcal{B}_{\text {triv }}$ is selfdual. Forgetting about supersymmetry for a moment, we can think of a monodromy defect line as the Dirac string associated to a magnetic monopole that may have been improperly quantized. Hence a monodromy defect line can end on a singular magnetic monopole (figure 27). Since monodromy defects are 


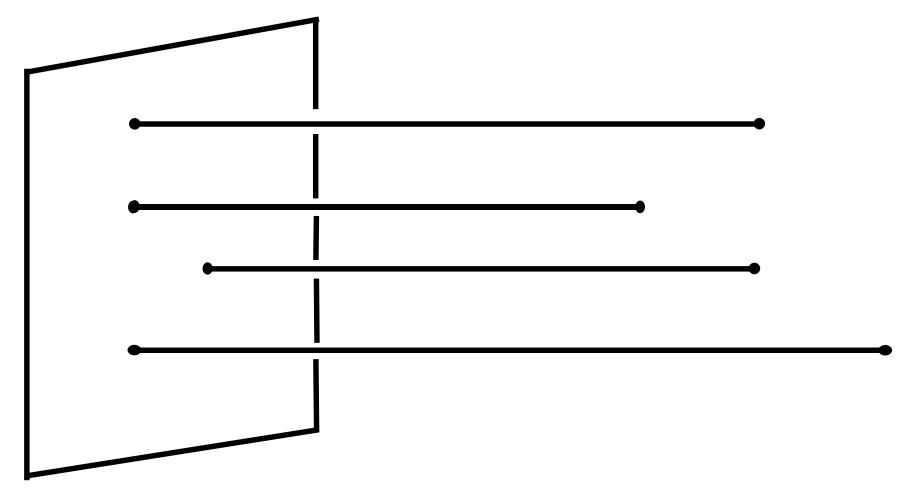

Figure 27: This figure differs from figure 26 only in that each monodromy defect line ends on a singular monopole, indicated by a black dot on the right. Since the defect lines themselves are selfdual (with a suitable transformation of their parameters), the brane defined by ending of the defect lines is also selfdual.

mapped to themselves by $S$-duality (with some transformation of the parameters), pictures in which the monodromy defects end on singular monopoles are similarly mapped to themselves by duality.

Supersymmetry imposes some constraints on the values of the parameters at which such pictures exist. In the context of the $B$-model of type $J$, the monodromy around a given defect line must be trivial if the defect line is going to end. This means that, in this $B$-model, the picture of figure 27 only exists if $\alpha=\gamma=0$ (here we will view $\alpha$ and $\eta$ as periodic variables). Of course, that is anyway the only case that the brane $\mathcal{B}_{\text {triv }}$ can be defined. Dually, in the $A$-model of type $\omega_{K}$, a picture like that of figure 27 only exists if $\gamma=\eta=0$. (For example, $\eta$ must vanish because the worldsheet thetaangle $\eta$ fails to preserve the topological supersymmetry of the $A$-model if the support of the monodromy defect ends at a place where the $U(1)$ bundle along the monodromy defect is not trivialized.)

In the context of the present paper, opers with trivial monodromy arise most directly from singular monopoles at $y=0$. However, without changing anything essential, we can move the singular monopoles away from the boundary as long as we connect them to the boundary via monodromy defects, as in figure 27. This has the advantage of making it obvious that opers with trivial monodromy are intersection points of two branes, and also making clear the selfduality of one of these branes.

In the general context of a defect line ending on a singular monopole, the monopole may be incorrectly quantized. However, for $\lambda=k / 2$, which is equivalent to $\gamma=0, \alpha=(k / 2) \operatorname{diag}(i,-i)$, the monopole at the end of the 
string obeys Dirac quantization, but the string is observable because we assume $\beta \neq 0$.

\subsection{Application to the Gaudin model}

The selfduality of the brane $\mathcal{B}_{\text {triv }}$ provides a gauge theory explanation of the main result of $[22,25]$ : opers on $\mathbb{C P}^{1}$ with trivial monodromy correspond to simultaneous eigenvectors of the commuting Hamiltonians of the Gaudin model. Let us consider the duality between the space of $\left(\mathcal{B}_{\mathrm{cc}}, \mathcal{B}_{\text {triv }}\right)$ strings and the space of $\left(\mathcal{B}_{\text {oper }}, \mathcal{B}_{\text {triv }}\right)$ strings. The following discussion assumes familiarity with the framework of [70].

To construct the space of $\left(\mathcal{B}_{\text {cc }}, \mathcal{B}_{\text {triv }}\right)$ strings, we have to quantize a moduli space $\left(\prod_{a=1}^{d} \mathbb{C P}_{a}^{1}\right) / S L(2, \mathbb{C})$, where $\mathbb{C P}_{a}^{1}$ is a copy of $\mathbb{C P}^{1}$ attached to the monodromy defect at $z=z_{a}$. Quantization of $\mathbb{C P}_{a}^{1}$ gives an irreducible representation $R_{a}$ of $S U(2)$ of spin $j_{a}=k_{a} / 2$, and quantization of $\left(\prod_{a=1}^{d} \mathbb{C P}_{a}^{1}\right) /$ $S L(2, \mathbb{C})$ gives a quantum Hilbert space $\mathfrak{H}$ that is the $S U(2)$-invariant part of $\otimes_{a} R_{a}$,

$$
\mathfrak{H}=\left(\otimes_{a} R_{a}\right)^{S U(2)}
$$

The classical commuting Hamiltonians of Hitchin's integrable systems can be interpreted (in the $A$-model of type $\left.\omega_{K}\right)$ as $\left(\mathcal{B}_{\mathrm{cc}}, \mathcal{B}_{\mathrm{cc}}\right)$ strings. So they act on the space $\mathfrak{H}$ of $\left(\mathcal{B}_{\text {cc }}, \mathcal{B}_{\text {triv }}\right)$ strings. In fact, the Hitchin Hamiltonians become the commuting Hamiltonians of the Gaudin model. To demonstrate the last statement, one interprets the generators of the $S U(2)$ action on $R_{a}$ as arising from first order differential operators on $\mathbb{C P}_{a}^{1}$, whence the Gaudin Hamiltonians (3.23) become second order differential operators. The "symbols" (or coefficients of the leading terms) of these operators are functions on the base of the Hitchin fibration that are precisely the Hitchin Hamiltonians. So, reading this in reverse, the Gaudin Hamiltonians represent a quantization of the Hitchin Hamiltonians (and this quantization is unique, given the commutativity of the Hitchin Hamiltonians, modulo the possibility of adding $c$-numbers).

To understand the eigenvectors and eigenvalues of the commuting Hamiltonians, we use the equivalence of $\left(\mathcal{B}_{\mathrm{cc}}, \mathcal{B}_{\text {triv }}\right)$ strings in the $A$-model of type $\omega_{K}$ to $\left(\mathcal{B}_{\text {oper }}, \mathcal{B}_{\text {triv }}\right)$ strings in the $B$-model of type $J$. The latter strings simply correspond to intersection points of the classical branes $\mathcal{B}_{\mathrm{cc}}$ and $\mathcal{B}_{\text {oper. }}$. So opers with trivial monodromy give a basis for the quantum Hilbert space $\mathfrak{H}$ of the Gaudin model. In the $B$-model description, the commuting Hamiltonians simply become functions on the variety $\mathcal{V}$ of opers, which is 


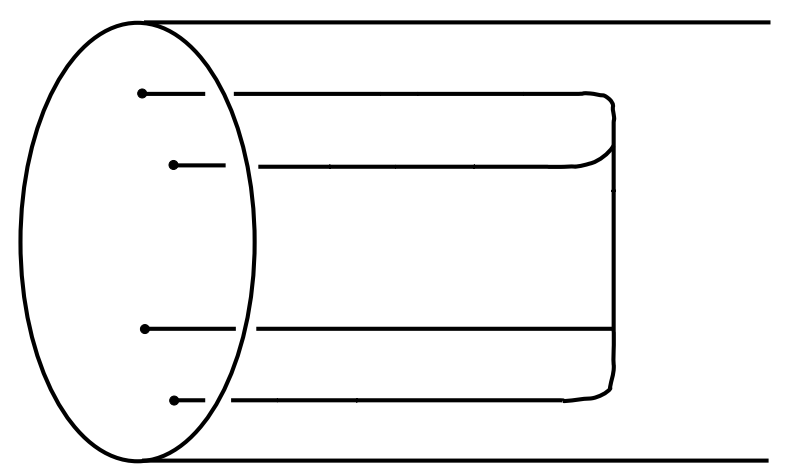

Figure 28: Another selfdual brane can be constructed by replacing the ends of monodromy defects, which we used in figure 27, with junctions of monodromy defects, as depicted here.

the support of the brane $\mathcal{B}_{\text {oper }}$. Hitchin's classical Hamiltonians are holomorphic functions on the space of quadratic differentials on $C$ (with poles of prescribed type at the positions $z_{a}$ of the monodromy defects). The support of $\mathcal{V}$ is the space of stress tensors on $C$ (with prescribed poles at the $z_{a}$ ). The space of stress tensors differs from the space of quadratic differentials only because of the $c$-number conformal anomaly. This matches the additive $c$-number ambiguity in the quantization of the Hitchin Hamiltonians. The eigenvalues of the quantized Hamiltonians corresponding to a given oper are simply given by the stress tensor associated to that oper.

Recently [66], a "noncompact" version of the Gaudin model has been described in which the finite-dimensional representations $R_{a}$ are replaced by infinite-dimensional ones. The eigenvectors of the commuting Hamiltonians are again expressed as opers, now with certain conditions on their monodromies. It is natural to suspect that this construction again reflects the existence of a selfdual brane. There actually is a good candidate - a selfdual brane that is constructed by replacing ends of monodromy defects, as in figure 27, by junctions of such defects, as in figure 28. Such a junction is defined by a solution of Hitchin's equations on a small two-sphere $S$ linking the junction with singularities (of a type depending on the parameters $\left.\alpha_{a}, \beta_{a}, \gamma_{a}, \eta_{a}\right)$ at the intersection points of $S$ with the monodromy defects.

\section{Acknowledgments}

We thank D. Bar-Natan, L. Kauffman, S. Lewallen, P. Li, R. Mazzeo, G. Moore, R. Schoen, P. Seidel, L.-F. Tam, and V. Toledano-Laredo for 
discussions and comments. We also thank M. Turansick for assistance with the figures.

\section{Appendix A Three-dimensional BPS equations from six dimensions}

The time-independent configurations we consider in Section 2 are solutions of the $3 \mathrm{~d}$ BPS equations

$$
\left[\mathcal{D}_{i}, \mathcal{D}_{j}\right]=0 \quad \sum_{i=1}^{3}\left[\mathcal{D}_{i}, \mathcal{D}_{i}^{\dagger}\right]
$$

for a $3 \mathrm{~d}$ connection together with three adjoint scalar fields, packaged together in the operators $\mathcal{D}_{i}$ as

$$
\begin{aligned}
& \mathcal{D}_{1}=\frac{D}{D x^{2}}+\mathrm{i} \frac{D}{D x^{3}} \\
& \mathcal{D}_{2}=\left[\phi_{2}-\mathrm{i} \phi_{3}, \cdot\right] \\
& \mathcal{D}_{3}=\frac{D}{D y}-\mathrm{i}\left[\phi_{1}, \cdot\right]
\end{aligned}
$$

Or, in a complex notation,

$$
\begin{aligned}
& \mathcal{D}_{1}=2 \frac{D}{D \bar{z}} \\
& \mathcal{D}_{2}=2[\varphi, \cdot] \\
& \mathcal{D}_{3}=\frac{\mathcal{D}}{\mathcal{D} y} .
\end{aligned}
$$

This system of equations can be generalized to a one-real-parameter family of 3d BPS equations, which can be written as in A.1, but with a different choice of operators $\mathcal{D}_{i}$ :

$$
\begin{aligned}
& \mathcal{D}_{1}=2 \frac{D}{D \bar{z}}+2 \zeta[\bar{\varphi}, \cdot] \\
& \mathcal{D}_{2}=-2 \zeta \frac{D}{D z}+2[\varphi, \cdot] \\
& \mathcal{D}_{3}=\frac{\mathcal{D}}{\mathcal{D} y} .
\end{aligned}
$$

The generalization was studied in Section 3 . 
This family of $3 \mathrm{~d}$ equations can be usefully derived from six dimensions. We start in $\mathbb{R}^{6}$ with coordinates $x^{a}, x^{a+3}, a=1,2,3$. Then we constrain a gauge field by requiring that the field strength, seen as an element of the $S O(6)$ Lie algebra, lies in a specified $S U(3)$ subgroup. As long as one is in six dimensions, the choice of a subgroup does not matter; it just amounts to the choice of an identification of $\mathbb{R}^{6}$ with $\mathbb{C}^{3}$. But if we require that the fields actually only depend on the first three coordinates $x^{a}$, and are invariant under constant shifts of $x^{a+3}$, then the choice of an $S U(3)$ subgroup does matter. So after dimensional reduction to three dimensions, one can obtain a family of inequivalent three-dimensional equations depending on a parameter.

A simple way to show that the family is of real dimension one, modulo equivalences, is as follows. First, a choice of embedding of $S U(3)$ in $S O(6)$, parameterized by $S O(6) / U(3) \sim S U(4) / U(3) \sim \mathbb{C P}^{3}$, is equivalent to the choice of a complex line in the space of $6 \mathrm{~d}$ spinors of positive chirality. After dimensional reduction to $3 \mathrm{~d}$, the inequivalent sets of $3 \mathrm{~d}$ equations are parameterized by such a choice modulo the $S O(3) \times S O(3)$ group of space rotations and rotations of the three scalars $\phi_{i}$. Although this group is six-dimensional, just like $\mathbb{C P}^{3}$, it does not act freely on $\mathbb{C P}^{3}$; rather, a generic point in $\mathbb{C P}^{3}$ preserves an $S O(2)$ subgroup of $S O(3) \times S O(3)$. For example, all the $3 \mathrm{~d}$ equations parameterized by $\zeta$ are invariant under a simultaneous phase rotation of $D / D z$ and $\phi_{z}$. In general, $S O(3) \times S O(3)$ acts as $S O(4) \subset S U(4)$ on the space of $6 \mathrm{~d}$ spinors. So a complex spinor of $S U(4)$ is a complex vector of $S O(4)$, and its real and imaginary parts break $S O(4)$ to $S O(2)$. Hence, the family of $3 \mathrm{~d}$ equations obtained as dimensional reduction of the $6 \mathrm{~d}$ equations is of real dimension one, and (A.4) is a generic representative.

A consequence of this picture is that we can change $\zeta$ by an $S O(6)$ rotation. Indeed, we can change $\zeta$ as desired by acting with an appropriate element of a group that we will call $S O(2)_{\zeta}$, which rotates $D / D x^{2}$ and $\left[\phi_{2}, \cdot\right]$ into each other, and also rotates $D / D x^{3}$ and $\left[\phi_{3}, \cdot\right]$ into each other. To be precise, the $S O(2)_{\zeta}$ rotation acting on the $\mathcal{D}_{i}$ defined at $\zeta=0$ will give a slightly rescaled version of the $\mathcal{D}_{i}$, with a prefactor $\left(1+\zeta^{2}\right)^{-1 / 2}$. This prefactor can be absorbed by a simple rescaling of the $z$ coordinate.

Now let us discuss the Nahm pole boundary condition that has been so important in the present paper. If we assume a dependence on $y$ only, and further assume that $A_{\bar{z}}=0$ (so that we can disregard $\mathcal{D}_{1}$ ), the equations (A.1) with the $\mathcal{D}_{i}$ defined as in (A.3) reduce to Nahm's equations. The Nahm pole boundary condition is defined by requiring that for $y \rightarrow 0$, the fields can be approximated by a certain singular solution of Nahm's equations. 
There is a similar boundary condition for the $3 \mathrm{~d}$ BPS equations at generic $\zeta$. Indeed, $S O(2)_{\zeta}$ maps a solution of (A.1) which only depends on $y$ to a solution of (A.4) which only depends on $y$. More explicitly, taking the general form of the $\mathcal{D}_{i}$ in (A.4), we can look for solutions, which depend on $y$ only, and such that $\mathcal{D}_{1}$ reduces to $2 \partial / \bar{\partial} \bar{z}$, i.e., $A_{\bar{z}}=-\zeta \bar{\varphi}$. Then $\mathcal{D}_{2}=$ $2\left(1+\zeta^{2}\right)[\varphi, \cdot]$ and hence we can embed solutions of the Nahm equations as solutions of the general 3d BPS equations, at the price of a rescaling of the complex scalar $\varphi$ by $1+\zeta^{2}$. This leads to the rotated Nahm pole boundary condition which we found useful in this paper.

Of course, what we have just described is not the only embedding of the Nahm pole which would be compatible with the general 3d BPS equations. For example, at $\zeta \neq 0$, we could have chosen to look for an embedding in which $\mathcal{D}_{2}$ rather than $\mathcal{D}_{1}$ is trivial; this would lead to what we might call antiopers - flat bundles with an oper-like constraint on their antiholomorphic structure, rather than on their holomorphic structure. Any rotation of our choice of Nahm pole by the $U(2)$ subgroup of $S U(3)$ which preserves $\mathcal{D}_{3}$ would produce a possible boundary condition, but we will generally stick to the "oper" Nahm pole.

We will conclude with an alternative explanation of the meaning of the parameter $\zeta$. For finite, nonzero $\zeta$, the $\mathcal{D}_{i}$ can be rescaled and interpreted as a generic complex $3 \mathrm{~d}$ connection. In Cartesian coordinates, we can denote the components of the connection as $\widetilde{\mathcal{D}}_{a}$. The complex equations tell us that the connection is flat. Then we have a moment map constraint, which sets to zero a certain constant linear combination of the commutators $\left[\widetilde{\mathcal{D}}_{a}, \widetilde{\mathcal{D}}_{b}^{\dagger}\right]$.

From this point of view, $\zeta$ only appears in the choice of moment map equation. A generic linear combination of the commutators is described by a $3 \times 3$ matrix of coefficients $\omega^{a b}$,

$$
\sum_{a, b} \omega^{a b}\left[\widetilde{\mathcal{D}}_{a}, \widetilde{\mathcal{D}}_{b}^{\dagger}\right]=0
$$

From equation (A.4), we have a useful relation: $\omega^{z \bar{z}} / \omega^{\bar{z} z}=\zeta^{2}$. If $\zeta^{2}=1$, $\omega^{a b}$ is symmetric, but in general that is not so.

Generically, under linear coordinate redefinitions, there is a onedimensional parameter space of possible $\omega^{a b}$. For example, if the symmetric part of $\omega^{a b}$ is positive definite, as it is for (A.4), we can make it into the identity matrix $\delta^{a b}$. Then the antisymmetric part $B^{a b}$ can be rotated to live in the $z, \bar{z}$ plane, and its magnitude is controlled by a single real parameter, which we can identify with $\zeta$. 


\section{Appendix B Three-dimensional BPS equations from four and eight dimensions}

In this appendix, we will discuss how the $3 \mathrm{~d}$ BPS equations of parameter $\zeta$ can arise from time-independent solutions of the four-dimensional BPS equations (1.1). We will generalize the statement that the $\zeta=0$ equations in three dimensions arise from the $4 \mathrm{~d}$ equations at $t=1$ if we drop the dependence on one coordinate, say $x^{1}$, and also set $A_{1}=\phi_{y}=0$.

First we will show that this is not a feature of a specific choice. We can start with any choice of $t$, set $d / d x^{1}=0$ and set $A_{1}$ and $\phi_{y}$ to any two linear combinations of the other three components of $\phi$, and the resulting $3 \mathrm{~d}$ equations will be equivalent to the $3 \mathrm{~d}$ BPS equations discussed in the last appendix for some value of the parameter $\zeta$ in (A.4).

For that purpose, it is rather convenient to rewrite the 4d BPS equations in a compact form, as a dimensional reduction of BPS equations in eightdimensional Yang-Mills theory. A succinct way to describe the desired eightdimensional equations is to pick a $\operatorname{Spin}(8)$ spinor $\epsilon$ of definite chirality and require

$$
F_{I J} \Gamma^{I J} \epsilon=0 .
$$

If the curvature $F_{I J}$ is understood as an element of the Lie algebra of $S O(8)$, then the equations restrict the curvature to a $\operatorname{Spin}(7)$ subalgebra of $S O(8)$. These are really 7 equations, because of the obvious relation

$$
\epsilon^{T} F_{I J} \Gamma^{I J} \epsilon=0,
$$

as $\Gamma^{I J}$ are antisymmetric.

Dimensional reduction to four dimensions breaks $S O(8)$ to a subgroup that we will call $S O(4)_{s} \times S O(4)_{R}$, acting respectively on the first four and last four coordinates. The spinor $\epsilon$ decomposes into a piece $\epsilon_{\mathrm{L}}$ which is left chiral under both $S O(4)_{s}$ and $S O(4)_{R}$, and a piece $\epsilon_{\mathrm{R}}$, which is right chiral under both $S O(4)_{s}$ and $S O(4)_{R}$. If both $\epsilon_{\mathrm{L}}$ and $\epsilon_{\mathrm{R}}$ are nonzero, they fix a choice of a twisted $S O(4)_{s}^{\prime}$ diagonally embedded in $S O(4)_{s} \times S O(4)_{R}$, such that $\epsilon_{\mathrm{L}}$ and $\epsilon_{\mathrm{R}}$ are $S O(4)_{s}^{\prime}$ scalars. Then the seven equations decompose under $S O(4)_{s}^{\prime}$ into a triplet of self-dual two-forms, a triplet of anti-self-dual forms and a scalar equation. This is the form familiar from (1.1).

We write $a^{\prime}$ as an abbreviation for $a+4$ and adopt a complex notation with $\underline{a}$ as an abbreviation for $a+\mathrm{i} a^{\prime}$ and $\bar{a}$ as an abbreviation for $a-\mathrm{i} a^{\prime}$. 
In order to bring the $8 \mathrm{~d}$ equations explicitly to the form (1.1), it is useful to combine the $\Gamma$ matrices to raising operators

$$
\gamma_{\underline{a}}=\Gamma_{a}+\mathrm{i} \Gamma_{a+4}
$$

and lowering operators

$$
\gamma_{\bar{a}}=\Gamma_{a}-\mathrm{i} \Gamma_{a+4}
$$

with $a=1, \ldots, 4$. We write $|\Omega\rangle$ for a state annihilated by the lowering operators, and $|\mho\rangle$ for its complex conjugate, a state annihilated by the raising operators. Being invariant under $S O(4)_{s}^{\prime}, \epsilon$ is a linear combination of $|\Omega\rangle$ and $|\mho\rangle$; being real, it is actually $\epsilon=\mathrm{e}^{-\mathrm{i} \alpha}|\Omega\rangle+\mathrm{e}^{\mathrm{i} \alpha}|\mho\rangle$, for some real $\alpha$.

Then the $8 \mathrm{~d}$ equations can be written in terms of the $(2,0),(1,1)$ and $(0,2)$ components of the curvature $F_{\underline{a b}}, F_{\underline{a} \bar{b}}$ and $F_{\bar{a} \bar{b}}$ :

$$
\begin{aligned}
\mathrm{e}^{-\mathrm{i} \alpha} F_{\underline{a b}}+\mathrm{e}^{\mathrm{i} \alpha} \frac{1}{2} \epsilon_{a b}{ }^{c d} F_{\bar{c} \bar{d}} & =0 \\
\sum_{a} F_{\underline{a} \bar{a}} & =0 .
\end{aligned}
$$

When we reduce to $4 \mathrm{~d}$, the first equation tells us that the selfdual part of $\operatorname{Re}\left(\mathrm{e}^{-\mathrm{i} \alpha} F_{a \underline{a b}}\right)$ vanishes, as does the anti-selfdual part of $\operatorname{Im}\left(\mathrm{e}^{-\mathrm{i} \alpha} F_{\underline{a b}}\right)$. With $\phi=\sum_{a} \overline{A_{a+4}} d x^{a}$, we recover the familiar $4 \mathrm{~d}$ equations

$$
\begin{aligned}
\left(F-\phi \wedge \phi+t d_{A} \phi\right)^{+} & =0 \\
\left(F-\phi \wedge \phi-t^{-1} d_{A} \phi\right)^{-} & =0 \\
d_{A} \star \phi & =0
\end{aligned}
$$

with $t=\tan \alpha$.

If we start from the $8 \mathrm{~d}$ form of the equations, it is clear that solutions which are independent of some of the eight directions preserve additional supersymmetry. For example, any solution such that $F_{I 8}=0$ for some $I$ also satisfies

$$
F_{I J} \Gamma^{I J} \Gamma^{8} \epsilon=0
$$

and hence preserves the supersymmetry generated by the real anti-chiral spinor $\Gamma^{8} \epsilon$ of $S O(8)$. The 7 equations remain independent, and describe a reduction of $S O(7)$ to $G_{2}$ preserving a $7 \mathrm{~d}$ spinor $\epsilon_{7}$. 
Solutions that satisfy $F_{I 8}=0$ and $F_{I 7}=0$ preserve generically four spinors: $\epsilon, \Gamma^{7} \epsilon, \Gamma^{8} \epsilon, \Gamma^{78} \epsilon$. The seven equations then describe the reduction of $S O(6)$ to $S U(3)$ preserving the supersymmetries generated by a $6 \mathrm{~d}$ complex spinor $\epsilon_{6}$ and its complex conjugate. They decompose into three complex equations and a real moment map condition

$$
\left[\mathcal{D}_{i}, \mathcal{D}_{j}\right]=0 \quad \sum_{i}\left[\mathcal{D}_{i}, \mathcal{D}_{\bar{i}}^{\dagger}\right]=0
$$

as discussed in Appendix A.

This is exactly the situation we are in whenever in the four-dimensional equations (B.6), for any value of $t$, we set $d / d x^{1}=0$ and set $A_{1}, \phi_{y}$ to any two linear combinations of the remaining three scalar fields $\vec{\phi}$ in $\phi$. Any such choices will produce a $3 \mathrm{~d}$ reduction of the $6 \mathrm{~d}$ BPS equations, and hence, according to the analysis in Appendix A, will be equivalent to the standard $3 \mathrm{~d}$ BPS equations for some $\zeta$. The 3d BPS equations admit the oper-Nahm pole boundary condition. This will induce a boundary condition in the original 4d BPS equations, which will be some deformation of the standard Nahm pole boundary condition. Vice versa, with this boundary condition, the usual vanishing theorems will guarantee that time-independent solutions arise from solutions of the corresponding 3d BPS equations.

Finally, we will describe a simple explicit choice of reduction from $4 \mathrm{~d}$ to $3 \mathrm{~d}$ which gives whatever $\zeta$ we wish. Starting from $\alpha=0$ and the standard reduction with $A_{1}=\phi_{y}=0$, we make simultaneous $S O(2)$ rotations in the $(a, a+4)$ planes for $a=1,2,3$, i.e., rotations of $D / D x^{a}$ and $\left[\phi_{a}, \cdot\right]$ into each other by angles $\theta_{a}$. (We do not make such a rotation for $x^{4}=y$, as this would not behave well when we introduce a boundary at $y=0$.) The rotation multiplies the creation and destruction operators by phases $\mathrm{e}^{ \pm \mathrm{i} \theta_{a} / 2}$, and hence the vacuum $|\Omega\rangle$ by the phase $\mathrm{e}^{-\mathrm{i} \sum_{a} \theta_{a} / 2}$. Hence it shifts the angle $\alpha$ by $\sum_{a} \theta_{a} / 2$, and acts correspondingly on the $t$ parameter.

In order to preserve the $S O(2)$ symmetry that rotates $x^{2}$ and $x^{3}$, it is natural to keep $\theta_{2}=\theta_{3}$. Given how the rotation transforms $\mathcal{D}_{2}$ and $\mathcal{D}_{3}$ in (A.3), we will then have clearly $\zeta=\tan \theta_{2}$.

Concerning the relation between $\theta_{1}$ and $\theta_{2}$, there are two particularly natural choices. If we want to keep three-dimensional topological symmetry along the boundary, we should keep $\theta_{1}=\theta_{2}=\theta_{3}=\theta$. A rotation by these angles will change $t$ to $\tan (3 \theta / 2+\pi / 4)$, and set $\zeta$ to $\tan \theta$. On the other hand, if we content ourselves with two-dimensional symmetry, we can keep $t=1$, by setting $\theta_{1}=-2 \theta_{2}=-2 \theta_{3}=-2 \theta$. Again, $\zeta$ will be $\tan \theta$. With 
this second choice, we deform only the Nahm pole boundary condition, and not the four-dimensional equations.

\section{Appendix C On boundary conditions and a special solution of the BPS equations}

Here we will describe the Nahm pole boundary condition for the 3d BPS equations with generic $\zeta$, allowing for singular monopoles on the boundary, and describe explicitly the model solution for the case of just one singular monopole. We work throughout on $\mathbb{R}^{2} \times \mathbb{R}$ (the generalization to $C \times \mathbb{R}_{+}$is straightforward).

We will write the BPS equations simply as a flatness condition

$$
\left[\widetilde{\mathcal{D}}_{i}, \widetilde{\mathcal{D}}_{j}\right]=0
$$

for a complex $3 \mathrm{~d}$ connection $\widetilde{\mathcal{D}}_{i}=d_{i}+\left[\mathcal{A}_{i}, \cdot\right]$ together with a moment map constraint. Just as in equation (A.4), the indices $i=1,2,3$ refer to $\bar{z}, z$, and $y$. The definition of the $\widetilde{\mathcal{D}}_{i}$ differs from equation (A.4) by a rescaling of $\mathcal{D}_{2}$.

This affects the relative normalization of the $\left[\widetilde{\mathcal{D}}_{i}, \widetilde{\mathcal{D}}_{i}^{\dagger}\right]$ terms in the moment map constraint. Of course, we can always rescale the $y$-coordinate with respect to $z, \bar{z}$. If we write the moment map constraint as

$$
\sum_{i, j} \omega^{i j}\left[\widetilde{\mathcal{D}}_{i}, \widetilde{\mathcal{D}}_{j}^{\dagger}\right]=0
$$

for a constant diagonal matrix $\omega^{i j}$, the statement invariant under scaling is that $\omega^{22}=\zeta^{2} \omega^{11}$. We will find it convenient to set $\omega^{11}=\zeta^{-2}, \omega^{22}=1$, $\omega^{33}=1$. If $\zeta^{2}=1$, then

$$
\omega^{i j} \partial_{i} \partial_{j}^{\dagger}=\partial_{y}^{2}+2 \partial_{z} \partial_{\bar{z}}
$$

is the Laplace operator for a Euclidean metric on the half-space $\mathbb{R}^{2} \times \mathbb{R}_{+}$ that is normalized in a slightly unconventional way

$$
d s^{2}=d y^{2}+2|d z|^{2} .
$$

This normalization will be useful later.

The flatness condition (C.1) tells us that $\widetilde{\mathcal{D}}_{i}=g \partial_{i} g^{-1}$ for a complex gauge transformation $g$, that is, a map from $\mathbb{R}^{2} \times \mathbb{R}_{+}$to $G_{\mathbb{C}}$. The moment map 
condition (C.2) is invariant under unitary ( $G$-valued) gauge transformations $g \rightarrow U g$. We can eliminate the gauge-invariance by introducing the gaugeinvariant Hermitian matrix $h=g^{\dagger} g$. Then the moment map equation can be conjugated to

$$
\omega^{i j} \partial_{i}\left(h^{-1} \partial_{j}^{\dagger} h\right)=0
$$

or

$$
\omega^{i j} \partial_{i} \partial_{j}^{\dagger} h=\omega^{i j}\left(\partial_{i} h\right) h^{-1}\left(\partial_{j}^{\dagger} h\right)
$$

When $\zeta^{2}=1$, this equation says that the map $h$ from the half-space $\mathbb{R}^{2} \times \mathbb{R}_{+}$ to the quotient space $G \backslash G_{\mathbb{C}}$ (endowed with its natural $G_{C}$-invariant metric $\left.\operatorname{Tr}\left(h^{-1} d h\right)^{2} / 2\right)$ is harmonic. Problems of this type are much-studied, but usually (for example, see [71]) in the context of a hyperbolic metric on $\mathbb{R}^{2} \times \mathbb{R}_{+}$, rather than a Euclidean metric, as in our case.

For simplicity, we will specialize to the case $G=S U(2)$, so that $G \backslash G_{\mathbb{C}}$ is a copy of hyperbolic three space $H^{3}$ or $\mathrm{AdS}_{3}$. We can write

$$
g=\left(\begin{array}{cc}
Y^{-1 / 2} & 0 \\
0 & Y^{1 / 2}
\end{array}\right)\left(\begin{array}{cc}
1 & -\Sigma \\
0 & 1
\end{array}\right)
$$

for a real function $Y$ and a complex function $\Sigma$. This is a general parametrization, in the sense that every $g \in S L(2, \mathbb{C})$ can be uniquely written in this form, modulo a unitary gauge transformation $g \rightarrow U g$. With this parametrization, we have

$$
h=\left(\begin{array}{cc}
Y^{-1} & -\Sigma Y^{-1} \\
-\bar{\Sigma} Y^{-1} & |\Sigma|^{2} Y^{-1}+Y
\end{array}\right) .
$$

In these coordinates, the natural metric on $H^{3}$ takes a familiar form

$$
\frac{1}{2} \operatorname{Tr}\left(h^{-1} d h\right)^{2}=\frac{d Y^{2}+d \Sigma d \bar{\Sigma}}{Y^{2}} .
$$

In general, in terms of the variables $Y$ and $\Sigma$, the equations for $h$ become

$$
\begin{aligned}
\omega^{i j}\left(\partial_{i}\left(Y^{-1} \partial_{j}^{\dagger} Y\right)+Y^{-2} \partial_{i} \Sigma \partial_{j}^{\dagger} \bar{\Sigma}\right) & =0 \\
\omega^{i j} \partial_{i}\left(Y^{-2} \partial_{j}^{\dagger} \bar{\Sigma}\right) & =0 .
\end{aligned}
$$

In the framework of Section 3.2, we want a boundary condition that is determined by the properties of the "small section". If we write $s$ for the 
small section in the complex gauge $\mathcal{A}_{i}=0$, then in the unitary gauge with $\mathcal{D}_{i}=g \partial_{i} g^{-1}$, the small section becomes $g s$. We must require $g s$ to go as $y^{1 / 2}$ as $y \rightarrow 0$, while $g$ itself diverges as $y^{-1 / 2}$. This means that $h$ diverges as $y^{-1}$ while $h s$ and $s^{\dagger} h$ are finite and $s^{\dagger} h s$ goes as $y$. The standard Nahm pole solution corresponds to $s=\left(\begin{array}{l}z \\ 1\end{array}\right)$ and

$$
g=\left(\begin{array}{cc}
y^{-1 / 2} & 0 \\
0 & y^{1 / 2}
\end{array}\right)\left(\begin{array}{cc}
1 & -z \\
0 & 1
\end{array}\right)
$$

This formula, which is familiar from equation (3.8), is equivalent to

$$
h=\left(\begin{array}{cc}
y^{-1} & -z y^{-1} \\
-\bar{z} y^{-1} & |z|^{2} y^{-1}+y
\end{array}\right) \text {. }
$$

Comparing to the general parametrization (C.8), we see that the standard Nahm pole solution is $Y=y, \Sigma=z$. (The normalization $\omega^{11}=\zeta^{-2}$, $\omega^{22}=1, \omega^{33}=1$ was chosen to ensure that this is a solution for all $\zeta$.) In other words, this solution is the "identity" map from the half-space $\mathbb{R}^{2} \times \mathbb{R}_{+}$ endowed with the Euclidean metric (C.4) to the half-space endowed with the hyperbolic metric (C.9). For $\zeta^{2}=1$, the assertion that this gives a solution is simply the statement that the "identity" map between half-spaces endowed with these two metrics is harmonic.

In general, if $s=\left(\begin{array}{l}P \\ Q\end{array}\right)$, we want to require that $Y \sim y$ and $P-Q \Sigma \sim y$ as $y \rightarrow 0$. The last statement means that if we set $\sigma(z)=P / Q$, then $\Sigma=\sigma$ at $y=0$. The fact that $Y \rightarrow 0$ for $y \rightarrow 0$ means that the boundary $y=0$ of the half-space $\mathbb{R}^{2} \times \mathbb{R}_{+}$is mapped to the conformal boundary at infinity of the hyperbolic space $H^{3}$. That conformal boundary is a copy of $\mathbb{C P}^{1}$. By adjoing $\mathbb{C P}^{1}$ to $H^{3}$, one makes the usual conformal compactification $\bar{H}^{3}$ of $H^{3}$. The choice of an oper without monodromy determines a holomorphic map $\sigma(z)$ from $\mathbb{R}^{2} \cong \mathbb{C}$ to $\mathbb{C P}^{1}$, and the condition $\left.\Sigma\right|_{y=0}=\sigma$ means that, as a map of the boundary of the half-space to $\mathbb{C P}^{1}, h$ coincides with $\sigma$. So our problem is this: given a holomorphic map $\sigma$ from the boundary of the halfspace to the conformal boundary of the hyperbolic space, we want to extend $\sigma$ to a map $h: \mathbb{R}^{2} \times \mathbb{R}_{+} \rightarrow \bar{H}^{3}$ that obeys (C.5) when restricted to $y>0$. For $\zeta^{2}=1$, we are simply trying to extend the given map $\sigma$ to a harmonic map from the half-space $\mathbb{R}^{2} \times \mathbb{R}_{+}$to $\bar{H}^{3}$. (Technically, we assume that the map $\sigma$ has only polynomial growth so that it extends to a holomorphic map from the one-point compactification of $\mathbb{C}$ to $\mathbb{C P}^{1}$, and we similarly require that $h$ extends to a continuous map from the one-point compactification of the half-space to $\bar{H}^{3}$.) 


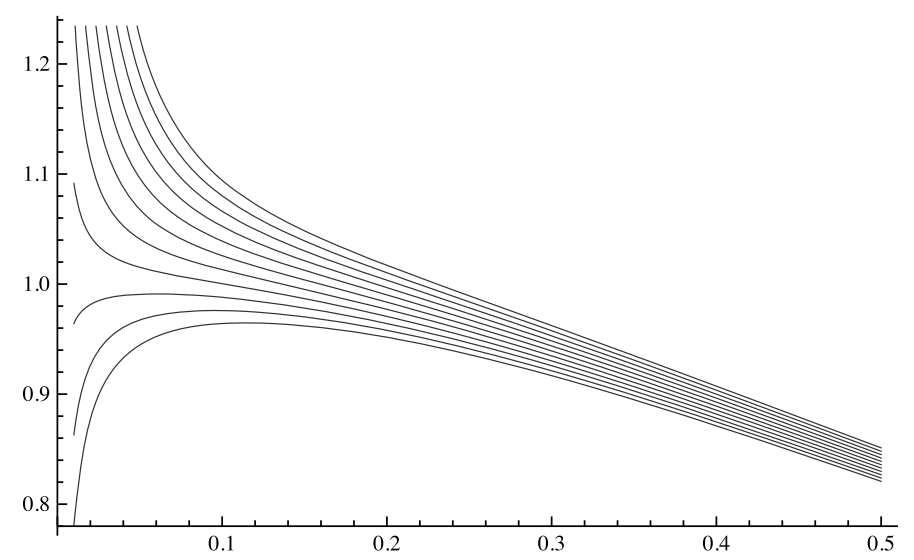

Figure 29: The numerical solutions as $\delta$ is varied across the critical value, for $k=1$.

For any $\sigma(z)$, at least away from the branch points of the map $\sigma$ - in other words, the zeroes of $d \sigma / d z$ - it is not difficult to write a systematic expansion of $Y$ and $Z$ for $y \rightarrow 0$, involving powers of $y$ and powers of $\log y$. The expansion roughly starts with $Y=y\left|\sigma^{\prime}(z)\right|+\cdots$ and $\Sigma=\sigma(z)+\cdots$, and the coefficients are rational functions in derivatives of $\sigma(z)$, and of three undetermined real functions of $z$ and $\bar{z}$. The denominators of these rational functions are powers of $\sigma^{\prime}(z)$ and its complex conjugate. So, away from the zeroes of $\sigma^{\prime}(z)$, boundary condition behaves well, and cuts in half the degrees of freedom of a solution. The branch points are precisely the points with $P Q^{\prime}-Q P^{\prime}=0$ - in other words, the points at which there are singular monopoles.

We still need to show that it is possible for a solution to be smooth away from the boundary in the presence of branch points or in other words singular monopoles on the boundary. The basic problem is to find a model solution in the presence of just one singular monopole; we then ask for the behavior near every singular monopole to match the model solution. In order to describe a singular monopole of charge $k$, we consider the special case $s=\left(\begin{array}{c}z^{k+1} /(k+1) \\ 1\end{array}\right)$, or in other words $\sigma(z)=z^{k+1} /(k+1)$. We also make use of the invariances of the BPS equations. The equations (C.10) are invariant under scale transformations $y \rightarrow \lambda y, z \rightarrow \lambda z$ with real $\lambda$, and under rotations $z \rightarrow \mathrm{e}^{\mathrm{i} \theta} z$. They are also invariant under reflections $z \rightarrow \bar{z}$ of $\mathbb{R}^{2}$, accompanied, if $\zeta^{2} \neq 1$, by $\Sigma \rightarrow \bar{\Sigma}$.

The boundary conditions $Y \sim 1 / y$ for $y \rightarrow 0,\left.\Sigma\right|_{y=0}=z^{k+1} /(k+1)$ are invariant under all these symmetries, accompanied by obvious rescalings of $Y$ and $\Sigma$ (which correspond to $S L(2, \mathbb{C}$ ) transformations of the hyperbolic 
space). We expect the solution of the moment map condition that obeys the boundary condition to be unique, so it must be invariant under all these symmetries. Hence we require $Y$ to be of the form $y|z|^{k} \mathrm{e}^{u(\rho)}$ and $\Sigma$ to be of the form $z^{k+1} \mathrm{e}^{u(\rho)} v(\rho) /(k+1)$, for real functions $u$ and $v$ of $\rho=y /|z|$.

Then the equations for $h$ turn into two unfortunately rather complicatedlooking nonlinear PDEs:

$$
\begin{aligned}
& v(\rho)\left(\zeta^{2}\left((4 k+3) \rho^{2}-8\right)-\rho^{2}\right) u^{\prime}(\rho)-4 k(k+1) \rho \zeta^{2} v(\rho) \\
& \quad+\rho\left(\rho^{2}+\left(\rho^{2}+4\right) \zeta^{2}\right) v(\rho) u^{\prime \prime}(\rho)-\left(\rho^{3}+\left(\rho^{2}+4\right) \rho \zeta^{2}\right) v(\rho) u^{\prime}(\rho)^{2} \\
& \quad+\rho\left(\rho^{2}+\left(\rho^{2}+4\right) \zeta^{2}\right) v^{\prime \prime}(\rho)+\left(-\rho^{2}-\left(\rho^{2}+8\right) \zeta^{2}\right) v^{\prime}(\rho)=0 \\
& \quad(k+1)^{2} \rho^{2}\left(\rho^{2}+\left(\rho^{2}+4\right) \zeta^{2}\right) u^{\prime \prime}(\rho) \\
& \quad+v^{\prime}(\rho)\left(2\left(\rho^{2}+\left(\rho^{2}+4\right) \zeta^{2}\right) v(\rho) u^{\prime}(\rho)-4(k+1) \rho \zeta^{2} v(\rho)\right) \\
& \quad+(k+1) \rho u^{\prime}(\rho)\left((k+1) \rho^{2}\left(\zeta^{2}+1\right)-4 \zeta^{2} v(\rho)^{2}\right)+4(k+1)^{2} \zeta^{2}\left(v(\rho)^{2}-1\right) \\
& \quad+\left(\rho^{2}+\left(\rho^{2}+4\right) \zeta^{2}\right) v(\rho)^{2} u^{\prime}(\rho)^{2}+\left(\rho^{2}+\left(\rho^{2}+4\right) \zeta^{2}\right) v^{\prime}(\rho)^{2}=0 .
\end{aligned}
$$

These equations involve $v$ and the first two derivatives of $u$ and $v$, but not $u$ itself. Indeed, a constant shift of $u$ is a symmetry of the equations, although not of the desired boundary conditions for $y \rightarrow 0$.

As PDEs for $v$ and the derivative $u^{\prime}$, these equations have a space of solutions which is locally three-dimensional. The requirement that the solution should be smooth as $z \rightarrow 0$ poses two constraints. It turns out that at large $\rho, u$ behaves as $k \log \rho$, so that $Y \sim y^{k+1}$, while $v(\rho)$ scales as $\rho^{-k}$, so that $\Sigma \sim \delta z^{k+1}$ for some constant $\delta$. The solution admits for large $\rho$ a convergent power series expansion in $1 / \rho$, which depends on $\delta$.

On the other hand, the boundary condition at $\rho \rightarrow 0$ is more forgiving, and only imposes a single further constraint on the solution, which basically reduces to the requirement that $v \rightarrow 1$ as $\rho \rightarrow 0$. It is not difficult to check numerically that $\delta$ can be tuned so that the solution satisfies the constraint, and it is hopefully possible to prove this rigorously for any nonzero finite $\zeta$. As $\delta$ is tuned, given the behavior for large $\rho$ imposed in the last paragraph, there are two possible behaviors for $v(\rho)$ as $\rho$ becomes small. If $\delta$ is small, $v(\rho)$ does not reach 1 , and goes to zero as $\rho \rightarrow 0$. If $\delta$ is large, it crosses 1 at some finite $\rho$, and then blows up before reaching $\rho=0$. The solution we are after corresponds to the critical value of $\delta$ which separates these two behaviors. 


\section{References}

[1] V.F.R. Jones, A polynomial invariant for links via Von Neumann algebras, Bull. AMS 12 (1985), 103.

[2] M. Khovanov, A categorification of the Jones polynomial, Duke. Math. J. 101 (2000), 359-426.

[3] S. Gukov, A.S. Schwarz and C. Vafa, Khovanov-Rozansky homology and topological strings, Lett. Math. Phys. 74 (2005), 53-74, arXiv:hep-th/0412243.

[4] H. Ooguri and C. Vafa, Knot invariants and topological strings, Nucl. Phys. B 577 (2000), 419, arXiv: hep-th/9912123.

[5] J.M.F. Labastida, M. Mariño and C. Vafa, Knots, links, and Branes at large N,' J. High Energy Phys. 11 (2000), 007, arXiv: hep-th/0010102.

[6] A. Neitzke and C. Vafa, Topological strings and their physical applications, arXiv:hep-th/0410178.

[7] M. Marinõ, Chern-Simons Theory, matrix models, and topological strings, Oxford University Press, 2005.

[8] N.M. Dunfield, S. Gukov and J. Rasmussen, The superpotential for knot homologies, Experiment. Math. 15 (2006), 129, arXiv:math/0505662.

[9] R. Dijkgraaf, C. Vafa and E. Verlinde, $M$-theory and a topological string duality, arXiv:hep-th/0602087.

[10] M. Aganagic and M. Yamazaki, Open BPS wall crossing and M-theory, Nucl. Phys. B 834 (2010), 258-272.

[11] S. Cecotti, A. Neitzke and C. Vafa, $R$-twisting and $4 d / 2 d$ corresondence, arXiv: $1006: 3435$.

[12] M. Aganagic, M.C.N. Cheng, R. Dijkgraaf, D. Krefl and C. Vafa, Quantum geometry of refined topological strings, arXiv:1105.0630.

[13] M. Aganagic and S. Shakirov, Knot homology from refined ChernSimons Theory, 2011, arXiv:hep-th/1105.5117.

[14] E. Witten, Fivebranes and knots, arXiv:1101.3216.

[15] A. Kapustin and E. Witten, Electric-magnetic duality and the geometric langlands program, Commun. Number Theor. Phys. 1 (2007), 1-236, arXiv:hep-th/0604151.

[16] P.B. Kronheimer, Instantons and the geometry of the nilpotent variety, J. Diff. Geom. 32 (1990), 473-490.

[17] A. Haydys, Fukaya-Seidel category and gauge theory, arXiv: 1010.2353.

[18] P.B. Kronheimer, M.Sc. thesis (Oxford University, 1986), unpublished. 
[19] D. Gaiotto and E. Witten, Supersymmetric boundary conditions in $\mathcal{N}=$ 4 super Yang-Mills theory, arXiv:08042907.

[20] N. Hitchin, The self-duality equations on a Riemann surface, Proc. London Math. Soc. 55 (3) (1987), 59-126.

[21] K. Corlette, Flat G-bundles with canonical metrics, J. Diff. Geom. 28 (1988), 361-382.

[22] E. Frenkel, Gaudin model and opers, arXiv:math/0407524.

[23] J. Teschner, Quantization of the Hitchin moduli spaces, Liouville theory, and the geometric Langlands correspondence I, arXiv:1005:2846.

[24] H.M. Babujian and R. Flume, Off-shell Bethe Ansatz equation for Gaudin magnets and solutions of Knizhnik-Zamolodchikov equations, Mod. Phys. Lett. A 9 (1994), 2029-2039.

[25] B. Feigin, E. Frenkel and N. Reshetikin, Gaudin model, Bethe Ansatz, and critical level, Commun. Math. Phys. 166 (1994), 27-62.

[26] A. Tsuchiya and Y. Kanie, Vertex operators in conformal field theory on $\mathcal{P}^{1}$ and monodromy representations of braid group, Adv. Stud. Pure Math. 16 (1998), 297-372.

[27] V.G. Knizhnik and A.B. Zamolodchikov, Current algebra and WessZumino model in two-dimensions, Nucl. Phys. B 247 (1984), 83-103.

[28] L.G. Rybnikov, Argument shift method and Gaudin model, Func. Anal. Appl. 40(3) (2006), arXiv:math.RT/0606380.

[29] B. Feigin, E. Frenkel and V. Toledano-Laredo, Gaudin models with irregular singularities, Adv. Math. 223 (2010), 873-948, arXiv:math/0612798.

[30] B. Feigin, E. Frenkel and L. Rybnikov, Opers with irregular singularity and spectra of the shift of argument subalgebra, arXiv:0712.1183.

[31] A.A. Belavin, A.M. Polyakov and A.B. Zamolodchikov, Infinite conformal symmetry in two-dimensional quantum field theory, Nucl. Phys. B 241 (1984), 333-380.

[32] Vl.S. Dotsenko and V.A. Fateev, Conformal algebra and multipoint correlation functions in 2D statistical models, Nucl. Phys. B 240 (1984), 312-348.

[33] G. Felder, BRST approach to minimal models, Nucl. Phys. B 317 (1989), 215.

[34] R.J. Lawrence, Homological representations of the Hecke algebra, Commun. Math. Phys. 135 (1990), 141-191.

[35] V.V. Schechtman and A.N. Varchenko, Arrangements of Hyperplanes and lie algebra homology, Invent. Math. 106 (1991), 139. 
[36] D. Gaiotto, Asymptotically free $\mathcal{N}=2$ theories and irregular conformal blocks, arXiv:0908.0307.

[37] T. Miwa, Clifford operators and Riemann's monodormy problem, Publ. Res. Inst. Math. Sci. Kyoto 17 (1981), 665.

[38] G.W. Moore, Matrix models of 2-D gravity and isomonodromic deformation, Prog. Theor. Phys. Suppl. 102 (1990), 255-286.

[39] M. Hutchings, Lecture notes on morse homology (with an eye towards Floer theory and pseudoholomorphic curves), available at math.berkeley.edu/ hutching/teach/276-2010/mfp.ps.

[40] E. Witten, Analytic continuation of Chern-Simons theory, arXiv: 1001.2933.

[41] E. Witten, A new look at the path integral of quantum mechanics, arXiv: 1009:6032.

[42] E. Witten, Supersymmetry and Morse theory, J. Diff. Geom. 17 (1982), 661-692.

[43] E. Frenkel, A. Losev and N. Nekrasov, Instantons beyond topological theory, I, arXiv:hep-th/0610149.

[44] E. Witten, Quantum field theory and the Jones polynomial, Commun. Math. Phys. 121 (1989), 351-399.

[45] H. Verlinde, Conformal field theory, two-dimensional gravity, and quantization of Teichmuller space, Nucl. Phys. B 337 (1990), 652-680.

[46] A. Bilal, W-algebras from Chern-Simons theory, Phys. Lett. B 267 (1991), 487-496.

[47] J. de Boer and J. Goeree, $W$ gravity from Chern-Simons theory, Nucl. Phys. B 381 (1991) 329-359.

[48] R. Lawrence, The Homological approach to higher representations, (Harvard preprint, 1990), available at http://www.ma.huji. ac.il/ ruthel/.

[49] L.H. Kauffman, Knots and physics, World Scientific, 1991.

[50] L.H. Kauffman, Map coloring, q-deformed spin networks, and TuraevViro invariants for 3-manifolds, Int. J. Mod. Phys. B 6 (1992), 1765-1794.

[51] E. Witten, Gauge theories, vertex models, and quantum groups, Nucl. Phys. B 330 (1990), 285-346.

[52] S. Albeverio and A.N. Sengupta, A mathematical construction of the non-Abelian Chern-Simons functional integral, Commun. Math. Phys. 186 (1997), 563-579.

[53] A. Hahn, The wilson loop observables of Chern-Simons on $\mathbb{R}^{3}$ in axial gauge, Commun. Math. Phys. 248 (2004), 467-499. 
[54] J.F.W.H. van de Wetering, Knot invariants and universal R-matrices from perturbative Chern-Simons theory in the almost axial gauge, Nucl. Phys. B 379 (1992), 172-198.

[55] J. Fröhlich and C. King, The Chern-Simons theory and knot polynomials, Commun. Math. Phys. 126 (1989), 167-199.

[56] M.F. Atiyah and N. Hitchin, The geometry and dynamics of magnetic monopoles, Princeton University Press, 1988.

[57] J. Hurtubise, Monopoles and rational maps: a note on a theorem of Donaldson, Commun. Math. Phys. 100 (1985), 191-196.

[58] S.J. Gates, C.M. Hull and M. Rocek, Twisted multiplets and new supersymmetric nonlinear sigma models, Nucl. Phys. B 248 (1984), 157.

[59] D.E. Diaconescu, D-Branes, monopoles and Nahm equations, Nucl. Phys. B 503 (1997), 220, arXiv:hep-th/9608163.

[60] M. Bershadsky, A. Johansen, V. Sadov and C. Vafa, Topological reduction of 4-d SYM to 2-d sigma models, Nucl. Phys. B 448 (1995), 166-186, arXiv:hep-th/9501096.

[61] J.A. Harvey, G.W. Moore and A. Strominger, Reducing $S$ duality to $T$ duality, Phys. Rev. D 52 (1995), 7161-7167, arXiv:hep-th/9501022.

[62] E. Frenkel, Lectures on the Langlands program and conformal field theory, arXiv:hep-th/0512172.

[63] N. Nekrasov and E. Witten, The omega deformation, Branes, Integrability, and Liouville theory, J. High Energy Phys. 09 (2010), 092, arXiv:1002.0888 [hep-th].

[64] L.F. Alday, D. Gaiotto and Y. Tachikawa, Liouville correlation functions from four-dimensional gauge theories, arXiv:0906.3219.

[65] N.A. Nekrasov and S.L. Shatashvili, Quantum Integrability and supersymmetric vacua, Prog. Theor. Phys. Suppl. 177 (2009), 105-119, arXiv:0901.4748 [hep-th].

[66] N. Nekrasov, A. Rosly and S. Shatashvili, Darboux coordinates, YangYang functional, and gauge theory, arXiv:1103.3919 [hep-th].

[67] C. Simpson, Harmonic bundles on noncompact curves, J. Am. Math. Soc. 3 (1990), 713-770.

[68] S. Gukov and E. Witten, Gauge theory, ramification, and the geometric langlands program, arXiv:hep-th/0612073.

[69] V. Mikhaylov, On the solutions of generalized Bogomolny equations, JHEP 1205 (2012), 112, arXiv:hep-th/1202. 4848.

[70] S. Gukov and E. Witten, Branes and quantization, arXiv:0809.0305.

[71] P. Li and L.-F. Tam, The heat equation and harmonic maps of complete manifolds, Invent. Math. 105 (1991), 1-46. 